University of Louisville

ThinkIR: The University of Louisville's Institutional Repository

$12-2013$

\title{
Psychosocial and environmental determinants of physical activity in elementary school children : implications for interventions to reduce childhood obesity.
}

Jiying Ling

University of Louisville

Follow this and additional works at: https://ir.library.louisville.edu/etd

\section{Recommended Citation}

Ling, Jiying, "Psychosocial and environmental determinants of physical activity in elementary school children : implications for interventions to reduce childhood obesity." (2013). Electronic Theses and Dissertations. Paper 836.

https://doi.org/10.18297/etd/836

This Doctoral Dissertation is brought to you for free and open access by ThinkIR: The University of Louisville's Institutional Repository. It has been accepted for inclusion in Electronic Theses and Dissertations by an authorized administrator of ThinkIR: The University of Louisville's Institutional Repository. This title appears here courtesy of the author, who has retained all other copyrights. For more information, please contact thinkir@louisville.edu. 
PSYCHOSOCIAL AND ENVIRONMENTAL DETERMINANTS OF PHYSICAL ACTIVITY IN ELEMENTARY SCHOOL CHILDREN: IMPLICATIONS FOR INTERVENTIONS TO REDUCE CHILDHOOD OBESITY

\author{
By \\ Jiying Ling \\ BSN, Shandong University, 2008 \\ MS, University of Louisville, 2013
}

\begin{abstract}
A Dissertation
Submitted to the Faculty of the School of Nursing of the University of Louisville in Partial Fulfillment of the Requirements for the Degree of
\end{abstract}

Doctor of Philosophy

School of Nursing University of Louisville Louisville, Kentucky

December 2013 
Copyright 2014 by Jiying Ling

All rights reserved 

PSYCHOSOCIAL AND ENVIRONMENTAL DETERMINANTS OF PHYSICAL ACTIVITY IN ELEMENTARY SCHOOL CHILDREN: IMPLICATIONS FOR INTERVENTIONS TO REDUCE CHILDHOOD OBESITY

\author{
By \\ Jiying Ling \\ BSN, Shandong University, 2008 \\ MS, University of Louisville, 2013
}

A Dissertation Approved on

November 25, 2013

By the following Dissertation Committee:

Barbara Speck (Dissertation Director), PhD, RN

Kristi King, PhD, CHES

Valerie Lander McCarthy, $\mathrm{PhD}, \mathrm{RN}$

Audra Rankin, DNP, RN

Joseph Petrosko, PhD 


\section{DEDICATION}

This dissertation is dedicated to my dear parents who have provided me unconditional love and invaluable support, and my brother, Weichen Ling, who shares my worry about my parents and lets me focus on academics. A special feeling of gratitude to my husband Fujun Wen for his love and support during the last two years in the $\mathrm{PhD}$ nursing program, and being always there for me.

I also dedicate this dissertation to my entire American family, Ann and Lou family, and Sandy and Rick family, for their unconditional support, encouragement and love, for always trusting me, and for considering me as one of their family members. They, as my family in America, are always there for me. They encourage me to move forward bravely, they care about my health as well as my achievements like my parents, and they love me like I am their daughter. 


\section{ACKNOWLEDGEMENTS}

I would like to first thank Dr. Barbara Speck, my dissertation chair, for her guidance, support and patience from the day I entered to the $\mathrm{PhD}$ nursing program through the day I defended the dissertation. She is a great mentor caring about my development by providing rigorous comments on each of my papers and suggestions on careers, and listening to my endless complaints. I really appreciate her patience and kindness to serve as my committee chair although she knew that she might need to spend more time on commenting on my work. Especially her encouragement for me to pursue the master degree in biostatistics, she builds the bridge from my thought to action. Everyone thinks that I am smart or wise to get the MS in biostatistics, but it is really her effort and 'push' that makes this happen.

I thank my committee members: Dr. Kristi King for opening the physical activity research area to me, for providing me many great research opportunities, and especially for her willingness to work with me; Dr. Joseph Petrosko for the guidance on statistical analysis and results interpretation; Dr. Audra Rankin for providing me valuable suggestions on pediatrics and childhood development; and lastly Dr. Valerie McCarthy for spending so much time on proofreading my proposal and dissertation, for providing me the great opportunity to work on her manuscripts, and for helping me gain teaching experience in lecturing, grading and clinical experience. Thank all committee members for serving in my dissertation committee, for bringing their intelligence and wisdom to my dissertation study, and for always being available for me. 
Especially, I want to take this opportunity to thank the executive director Ms. Cathy Graninger in the Communities In Schools of Clark County for providing me the access to the elementary school children attending afterschool programs. Her support on research and her concern on the current childhood obesity epidemic helped me to recruit adequate number of children in their afterschool programs. She is a great woman caring about children's academic performance as well as their healthy behaviors. She also provides me great suggestions on the incentives to motivate children to participate and retain in the dissertation study. I would also like to thank all the staff in the afterschool programs for their help, support and encouragement during the data collection time.

Lastly, I want to thank all the faculty, staff and students in School of Nursing, University of Louisville for making me feel home here. I am very pride of being a student in School of Nursing $\mathrm{U}$ of $\mathrm{L}$, and thank them all for developing this great $\mathrm{PhD}$ nursing program. I also thank U of L Commission on Diversity and Racial Equality, Sigma Theta Tau International-Iota Zeta Chapter, U of L School of Nursing Community Engagement, and $\mathrm{U}$ of L Graduate Student Council for their generous financial support on the dissertation study. 


\title{
ABSTRACT \\ PSYCHOSOCIAL AND ENVIRONMENTAL DETERMINANTS OF PHYSICAL ACTIVITY IN ELEMENTARY SCHOOL CHILDREN: IMPLICATIONS FOR INTERVENTIONS TO REDUCE CHILDHOOD OBESITY
}

\author{
Jiying Ling
}

November 25, 2013

Introduction: Childhood obesity has become a national public health crisis in America. Physical activity has been shown to be one key for controlling childhood obesity, but little is known about the mediators and moderators in physical activity promotion models. In particular, the immediate time afterschool provides an important opportunity for children to be active, only $20 \%$ of their afterschool time is used for physical activity.

Purpose: The purposes of this study were to explore the psychosocial and environmental determinants of physical activity, and to examine the influence of wearing pedometers on the hypothesized determinants (physical activity self-efficacy, physical activity enjoyment, perceived parental influence, and perceived environment) of physical activity, among elementary school children attending afterschool programs,

Methods: A cross-sectional correlational design was used to explore the determinants of physical activity in 133 children enrolled in afterschool programs. In addition, a pretest-posttest study was conducted to examine the influence of wearing pedometers on the hypothesized determinants of physical activity among a subsample of 
50 randomly selected children. Children's physical activity levels were assessed by a seven-day recall scale, and seven consecutive days' pedometer steps.

Results: Approximately $49 \%$ of the children were overweight or obese, but only $13 \%$ of the children met national physical activity recommendations. Overweight or obese children took fewer pedometer steps than non-overweight children, and pedometer steps were negatively related to children's BMI. Wearing pedometers did not have a significant influence on the hypothesized determinants of physical activity. No evidence of the reactivity of wearing pedometers was found. Perceived parental influence partially mediated the relationships of physical activity self-efficacy and physical activity enjoyment with physical activity. For self-reported physical activity levels, physical activity enjoyment had the strongest effect on physical activity followed by physical activity self-efficacy. Perceived parental influence had the strongest effect on physical activity measured by pedometers.

Conclusions: Pedometers are a reliable measurement instrument to assess elementary school children's physical activity. Perceived parental influence has the primary effect on children's self-reported physical activity levels and number of pedometer steps. Future physical activity interventions should target parental influence to improve children's physical activity levels and control childhood obesity. 


\section{TABLE OF CONTENTS}

\section{PAGE}

ACKNOWLEDGEMENTS ...................................................... iv

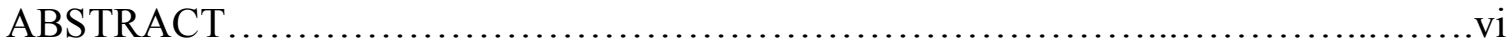

LIST OF TABLES .................................................................

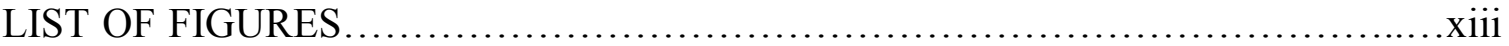

\section{CHAPTER I INTRODUCTION}

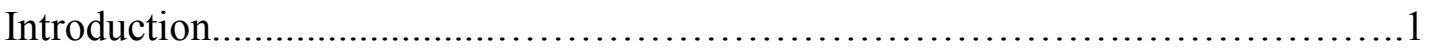

Statement of the Problem...................................................... 6

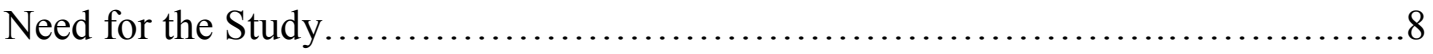

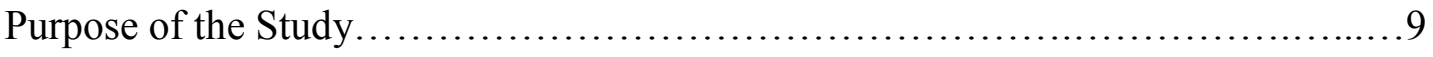

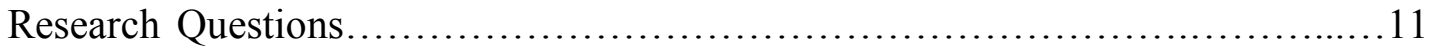

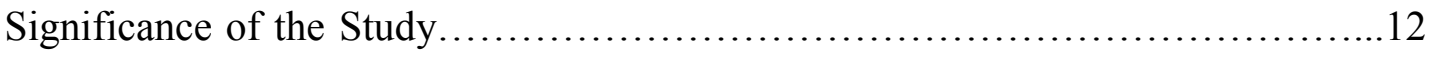

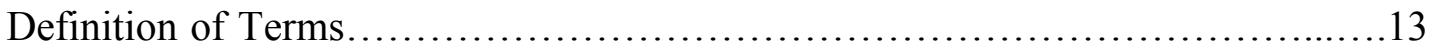

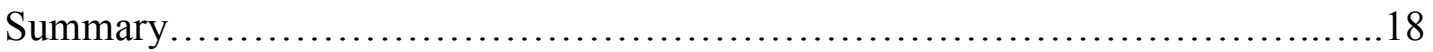

\section{CHAPTER II BACKGROUND AND SIGNIFICANCE}

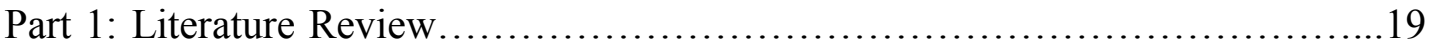

Elements and Patterns of Physical Activity...............................19

Physical Activity Measurements.......................................22

Physical Activity in Children.........................................25

Correlation between Physical Activity and Childhood Obesity.................29 
Physical Activity Interventions in Children..............................31

Determinants of Physical Activity in Children.............................35

Part 2: Conceptual Framework..........................................44

Rational for Choosing YPAP Model...................................44

Precede-Proceed Health Promotion Planning Model..........................53

Youth Physical Activity Promotion Model................................56

Empirical Uses of YPAP Model.........................................58

Conceptual Framework for the Dissertation Study.......................60

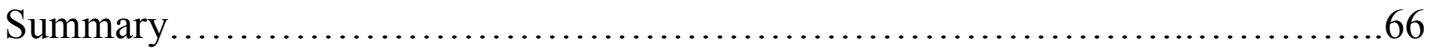

\section{CHAPTER III METHODOLOGY}

Research Design........................................................69

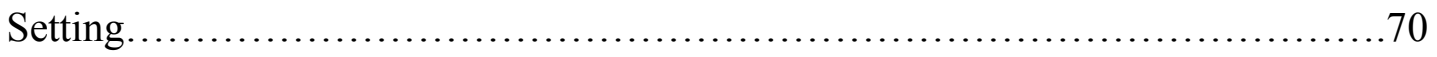

Sample Size......................................................... 73

Sample................................................................. 74

Variables and their Measurements........................................... 79

Personal Demographics................................................80

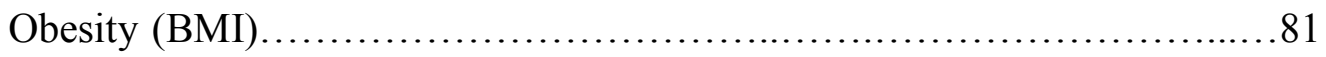

Outcome Variable.....................................................81

Predisposing Factors.............................................84

Reinforcing Factor.............................................. 88

Enabling Factor..................................................... 89

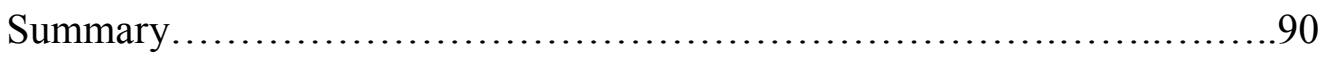

Data Collection.........................................................92 
Data Analysis........................................................ 95

Data Cleaning......................................................95

Instruments Coding............................................96

Reliability and Validity Checks...................................97

Assumptions of Structural Equation Modeling............................98

Preliminary Analysis.............................................100

Primary Analysis................................................101

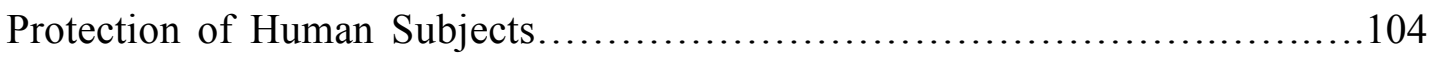

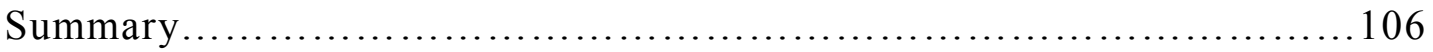

\section{CHAPTER IV RESULTS}

Scale Construction and Psychometric Characteristics.........................108

Compliance Rate, Reliability, and Reactivity of Wearing Pedometers.............118

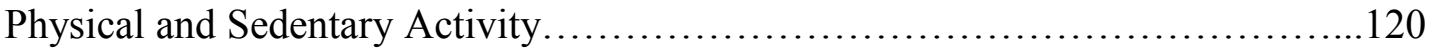

Obesity Status........................................................ 123

Determinants of Physical Activity....................................... 125

Research Questions.................................................. 126

Summary of Key Findings........................................... 144

\section{CHAPTER V DISCUSSION AND CONCLUSIONS}

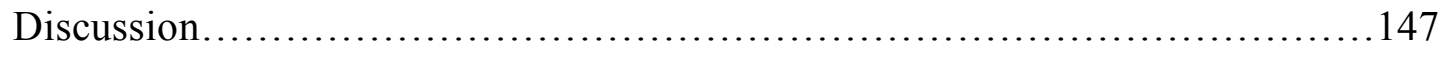

Scale Construction and Psychometric Characteristics.....................147

Compliance Rate, Reliability, and Reactivity of Wearing Pedometers........152

Sample Characteristics.......................................... 155

Obesity Status................................................ 156 
Physical Activity ..................................................... 158

Obesity Status and Physical Activity....................................162

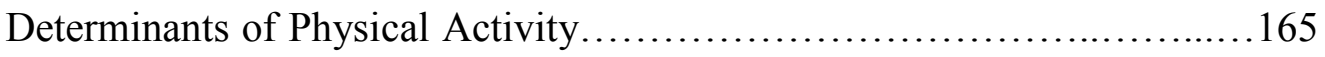

Implications for Research, Practice, and Policy...............................172

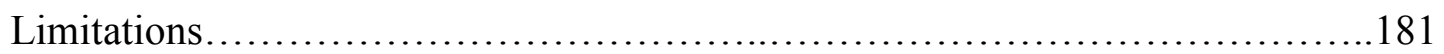

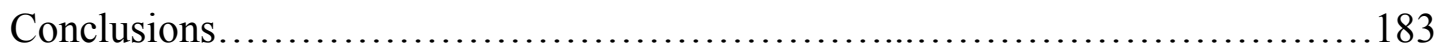

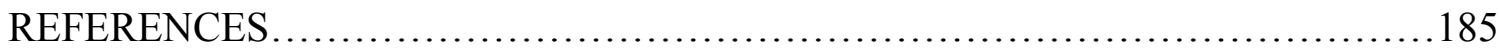

\section{APPENDICES}

A Reliability and Validity of Self-report Instruments for

Assessing Physical Activity in Children................................223

B Demographic Information Questionnaire.................................229

C Boys Body Mass Index-for-age Percentiles..............................231

D Girls Body Mass Index-for-age Percentiles............................232

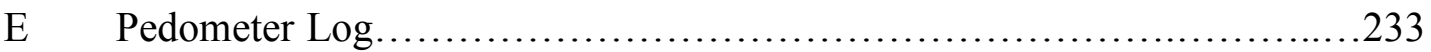

F Physical Activity Questionnaire for Older Children.........................235

G Physical Activity Self-Efficacy Scale.................................240

H Physical Activity Enjoyment Scale....................................2242

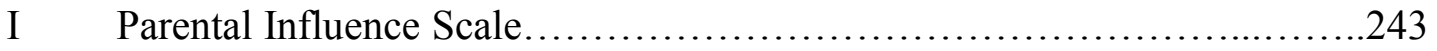

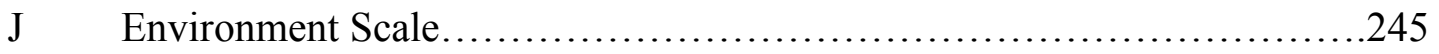

K Hierarchical Regression Analysis Evaluating Predictors of

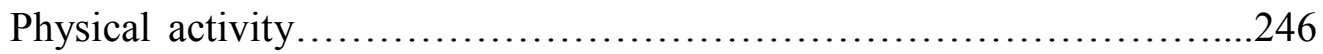

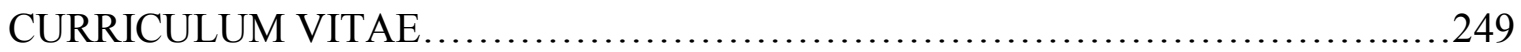




\section{LIST OF TABLES}

$\begin{array}{lll}\text { TABLE } & \text { PAGE }\end{array}$

1. Comparisons of Conceptual and Operational Definitions between the YPAP Model and the Dissertation Conceptual Framework....................64

2. Demographic Summary of Communities.................................... 72

3. Demographic Characteristics of Participants................................ 77

4. Instruments to Operationalize Variables...................................91

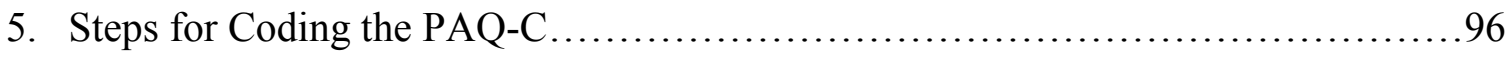

6. Psychometric Properties of the Major Study Variables............................111

7. Factorial Structure Matrix Rotated to the Varimax Criterion for PASES-C.........113

8. Factorial Structure Matrix Rotated to the Varimax Criterion for PACES...........115

9. Factorial Structure Matrix rotated to the Varimax Criterion for PAIS...............116

10. Environment Scale Factorial Structure Matrix Rotated to the Varimax Criterion...118

11. Pedometer Reliability over Seven Consecutive Days...........................119

12. Mean and Standard Deviation of Pedometer Steps over Time....................120

13. Effects of Wearing Pedometers on the Determinants of Physical Activity...........126

14. Interrelationships among Variables..................................... 128

15. Model Fit Indices of Theoretical Model, Model A, Model B,

Model C, Model D, Model E, and Model F................................... 136

16. Model Fit Indices of Theoretical Model, Model A, Model B,

Model C, Model D, Model E, and Model F 


\section{LIST OF FIGURES}

$\begin{array}{ll}\text { FIGURE PAGE } & \text { PAR }\end{array}$

1. The Youth Physical Activity Promotion Model .................................58

2. The Conceptual Framework for the Study, Adapted from the YPAP Model..........63

3. The Hypothesized Path Model............................................. 74

4. Power Analysis for Paired-samples t-test.................................. 74

5. Scatterplot between Self-reported Physical Activity Levels and Pedometer Steps...128

6. Self-Reported Physical Activity Models................................... 133

7. Pedometer Steps Models.............................................. 140 


\section{CHAPTER I}

\section{INTRODUCTION}

Childhood obesity is a growing global health problem of epidemic proportions.

Between the 1980s and 1990s, the prevalence of overweight and obesity among children increased by a magnitude of two to five times in developed countries, and up to four times in developing countries (Lobstein, Baur, Uauy, \& Iaso International Obesity TaskForce, 2004). Since 1970, the greatest annual increases in obesity among schoolaged children occurred in the United States (U.S.) and Western Europe (Wang \& Lobstein, 2006). Childhood obesity is a national public health crisis in the U.S., with significant sex, age, and ethnic disparities. Results from the National Health and Nutrition Examination Survey (NHANES) indicate that between 1963 and 2008, childhood obesity rates have tripled, with obesity rates being disproportionately higher among children aged 6 to 11 years old (Ogden, 2010). Today, nearly one in three children in the U.S. is overweight or obese (The White House: Office of the First Lady, 2010). Current evidence suggests that the prevalence of childhood obesity is likely to remain on the rise, and it was estimated that by the year 2030 , more than $60 \%$ of American children and nearly $90 \%$ of American adults would be overweight or obese (Wang, Beydoun, Liang, Caballero, \& Kumanyika, 2008).

Childhood obesity not only can directly influence children's physical and psychological health (short-term effects), but also can influence subsequent adult health (long-term effects) (Engeland, Bjorge, Sogaard, \& Tverdal, 2003; Lobstein et al., 2004; 
Reilly et al., 2003). Short-term effects of childhood obesity can be categorized into psychological consequences and physical consequences. Evidence has concluded that obese children are more likely to experience psychological problems, such as social isolation, social stigma, ridicule, and discrimination, than non-obese children (Reilly et al., 2003). Moreover, obese children tend to have lower self-esteem, and higher rates of sadness, loneliness and nervousness than non-obese children (Strauss, 2000; Wang \& Veugelers, 2008). As a result, obese children are more likely to engage in high-risk behaviors such as smoking or consuming alcohol. For physical consequences, results from previous studies have consistently supported that childhood obesity is significantly correlated with a range of adverse metabolic and cardiovascular traits - hypertension, dyslipidemia, abnormalities in left ventricular mass and/or function, abnormalities in endothelial function, and hyperinsulinemia/insulin resistance (Reilly, 2005; Saha, Sarkar, \& Chatterjee, 2011). Expert committee reports and reviews have recommended that childhood obesity should be considered as an important component of cardiovascular risk (Williams et al., 2002). Therefore, these psychological and physical consequences result in decreased health-related quality of life among overweight or obese children (Olshansky et al., 2005).

Childhood obesity not only has short-term effects, but will also have long-term effects as the current generation of obese children becomes adults. It has been estimated that two in three children born after 2000 will suffer from obesity-related health problems like cardiovascular disease, diabetes, hypertension, cancer, gall bladder disease, osteoarthritis, endocrine disorders and asthma at some point in their lives (Lobstein et al., 2004). Evidence indicates that more than $70 \%$ of obese children will become obese adults 
(Reilly et al., 2003). Because of the persistence of obesity, current cohort of American children are projected to have a shorter life expectancy than their parents, and the trend of rising life expectancy in the past two centuries will end soon (Olshansky et al., 2005). The economic costs of childhood obesity are especially evident in health care expenditures (Wang \& Dietz, 2002; Wang et al., 2008). Obesity-associated annual hospital costs increased from \$35 million during 1979-1981 to \$127 million during 19971999 (Wang \& Dietz, 2002). In 2011, health care costs of obesity-related diseases were estimated at about $\$ 147$ billion per year, and by 2030 , health care costs attributed to overweight and obesity will account for $\$ 900$ billion per year, that is, one in every six dollars spent on health care (Wang et al., 2008). Thus, studies related to childhood obesity prevention and interventions are urgently needed.

Considering the increasing childhood obesity epidemic, many efforts have been made to explore the determinants (influencing factors) of childhood obesity in order to control obesity effectively in children. Unhealthy behaviors (physical inactivity and unhealthy eating) are the two main determinants of the childhood obesity epidemic in the U.S. (Slyper, 2004). Some researchers suggest that physical activity should be the key in controlling childhood obesity (Nowicka \& Flodmark, 2007), but one recent study found that only about $1 \%$ of children in rural Kentucky met physical activity recommendations (13,000 steps/per day for boys and 11,000 steps/per day for girls) (Ling, King, Speck, Kim, \& Wu, 2013, in press). Therefore, this study focused on the determinants of physical activity to further provide implications for potential interventions to reduce childhood obesity. 
Lack of physical activity has been reported to be a significant contributor to childhood obesity, and physical activity is a promising factor in controlling childhood obesity (Hills, Andersen, \& Byrne, 2011; Janssen et al., 2005). Consistent evidence indicates that overweight or obese children participate in lower levels of physical activity, and higher levels of sedentary activity (screen-viewing time such as watching TV or playing video games) compared to normal weight children (Hills et al., 2011; Nowicka \& Flodmark, 2007). Children with higher levels of physical activity have reduced skinfold thickness (Davis et al., 2007). Greater physical activity participation in elementary school age children is significantly correlated with lower odds of overweight or obesity (Janssen et al., 2005), while sedentary lifestyle is a significant determinant of elevated Body Mass Index (BMI) (Bukara-Radujkovic \& Zdravkovic, 2009). Therefore, increasing physical activity participation should be one focus of strategies to control obesity in children.

Physical activity influences body weight in two ways. Increased physical activity directly influences weight by causing the body to mobilize and burn body fat to meet increased metabolic needs. Second, physical activity changes body composition by increasing lean body mass, which can increase the capacity of mobilizing muscles to burn body fat (Nowicka \& Flodmark, 2007). Weight is gained when energy intake exceeds energy expenditure. In order to maintain a stable weight, a delicate balance between energy intake and energy expenditure is required. Generally, energy expenditure comprises three components: resting energy expenditure (60-75\%), the thermic effects of food (the energy expended in consuming and processing food, 10\%), and the thermic effects of physical activity (15-30\%). In addition to these three components of energy expenditure, children need additional energy for growth. The required energy need for 
growth is about 1-2\% of total energy expenditure in childhood (Steinbeck, 2001). The resting energy expenditure is the minimum energy needed to sustain life, and it remains relatively constant within individuals over time (Goran \& Treuth, 2001). The thermic effects of food are primarily influenced by the composition of food, and are relatively stable within individuals over time (Steinbeck, 2001). The thermic effects of physical activity are the most variable component of energy expenditure and vary within and between individuals due to the variable nature of physical activity (Goran \& Treuth, 2001). Thus, physical activity is the discretionary component of energy expenditure.

Though physical activity is a promising factor in controlling childhood obesity, only $48 \%$ boys and $35 \%$ girls meet the daily physical activity recommendation of at least 60 minutes of moderate- to vigorous-intensity physical activity daily in the U.S. (Troiano et al., 2008). Physical education offers a great opportunity to provide all children physical activity, and teach them the knowledge and skills needed to establish active lifestyles. However, only three states meet the national recommendation for physical education in schools -150 minutes of instructional physical education per week for elementary school children and 225 minutes for middle and high school students (National Association for Sport and Physical Education [NASPE], 2010), and no more than 4\% of elementary schools actually provide daily physical education to students (Lee, Burgeson, Fulton, \& Spain, 2007).

In summary, childhood obesity has become a national public health crisis in the U.S. Overweight or obese children engage in lower levels of physical activity compared to non-overweight children, and lack of physical activity is a significant contributor to childhood obesity. Though physical activity is a promising factor in controlling childhood 
obesity, the majority of American children do not meet daily physical activity recommendations and children spend more time watching TV than participating in physical activity.

\section{Statement of the Problem}

Considering the strong negative relationship between physical activity and childhood obesity, many physical activity interventions have been designed and conducted to increase children's physical activity levels and to control childhood obesity. However, the majority of physical activity interventions remain largely ineffective in reducing obesity indices among children such as BMI and body fat mass. Specifically, physical activity interventions, with durations ranging from 6 months to 3 years, have shown no significant effect on decreasing BMI, percent body fat, total body fat mass, waist circumference, waist-to-hip ratio, skin-fold thickness, pulse rate, systolic or diastolic blood pressure (Dobbins, De Corby, Robeson, Husson, \& Tirilis, 2009; Harris, Kuramoto, Schulzer, \& Retallack, 2009).

Ineffectiveness of physical activity interventions may be attributed to an insufficient dose of physical activity such as short duration of interventions, insufficient quantity of physical activity, or low adherence to intervention protocols (Dobbins et al., 2009; Harris et al., 2009). Beyond the dose of physical activity, the effectiveness of physical activity interventions may depend on the measurement of physical activity, study design, intervention methods, and evaluation methods (Steinbeck, 2001).

The role of theory in planning interventions is to increase understanding of a phenomenon and to guide decisions such as dose of interventions and protocols for testing effectiveness of interventions. Evidence shows that understanding the 
determinants of physical activity in children is essential for building a framework to guide future physical activity intervention designs (Steinbeck, 2001). To design effectively tailored physical activity interventions to promote physically active lifestyles and control childhood obesity, a theoretical framework that considers determinants of physical activity in children is necessary. However, many widely used theories of physical activity have limitations when applied to physical activity interventions among children.

Theories used to guide physical activity interventions are subject to various limitations. Models of individual health behavior, such as Theory of Planned Behavior (TPB) and Self-Determination Theory (SDT), cannot inform the development of physical activity interventions that target changes beyond the individual level. Social Cognitive Theory (SCT), a model of interpersonal health behavior, recognizes the influence of the environment on behavior, but only focuses on peoples' potential abilities to change and construct environment to meet their needs (Glanz, Rimer, \& Viswanath, 2008). Children's behaviors are more passively affected by the environment, because they have little power to control their lives and change their environments. Though community and group models of health behavior change, such as social ecological models, recognize multiple influences on healthy behaviors, such as environment and support, these models do not provide testable hypotheses and specific guidelines about which variables are most important for physical activity among children (Elder et al., 2007).

For a model to effectively guide research on behavior change, the model must be tailored to specific populations and behaviors (Sallis et al., 2006). However, few theories and models currently used to guide research on physical activity were developed 
specifically for children (Welk, 1999). Most studies in children have applied models that were developed for adults, with the assumption that the models would be effective for children (Welk, 1999). It is the premise of this study that it is premature to assume that behaviors of children, adolescents and adults are influenced in the same way.

\section{Need for the Study}

The Youth Physical Activity Promotion (YPAP) model, based on the PrecedeProceed Health Promotion Planning Model (Green \& Kreuter, 2005) as an organizational framework for health promotion, was developed specifically for promoting physical activity among children and adolescents by considering their developmental, psychological, and behavioral characteristics (Welk, 1999). According to the YPAP model, there are three primary determinants of physical activity (predisposing factors, reinforcing factors, and enabling factors) among children and adolescents. Like the SCT and social ecological models for health behaviors, the YPAP encompasses both individual-level and environmental-level factors. In other words, this model merges SCT with social ecological models composed of similar or shared elements, which should further enhance the predictive ability of the YPAP model (Lox, Martin-Ginis, \& Petruzzello, 2006). Additionally, it yields testable hypotheses, so the model is testable and practicable. In all, the population- and behavior-specific YPAP model should be expected to have greater power to predict physical activity in children.

However, while evidence supports the relationships among predisposing factors, reinforcing factors, enabling factors and physical activity as proposed in the YPAP model among adolescents, only one study has been found that applies this model to children (Rowe, Raedeke, Wiersma, \& Mahar, 2007). According to Piaget (1952), children do not 
develop adult-like capacity for abstract thought and reason until age 11-14, which implies that children's behaviors might be motivated and influenced in different ways than adolescents. The only study using this model as a theoretical framework among children focused on the influence of predisposing factors and reinforcing factors on physical activity, but did not examine enabling factors such as environment (Welk, Wood, \& Morss, 2003). Thus, it is very important to explore the YPAP model in children to understand the determinants of physical activity - predisposing factors, reinforcing factors, and enabling factors - to provide valuable information for future effective physical activity interventions among children to promote physical activity and control childhood obesity.

\section{Purpose of the Study}

The purpose of this study was to explore psychosocial and environmental determinants of physical activity among children aged 8 to 11 years old by examining relationships among predisposing factors (physical activity self-efficacy and physical activity enjoyment), reinforcing factors (parental influence), enabling factors (environment) and physical activity levels. Children aged 8 to 11 years old were chosen for this study because: (a) obesity rates are higher among children aged 6 to 11 years old than other groups of children (Ogden, 2010); (b) physical activity levels in American children drop sharply between age 9 and age 15 (Bock \& Miller, 2008); and (c) 8 year old, children can read with considerable ease and can use complex and compound sentences (Rodgers, 2007).

Variables selected as predisposing factors, reinforcing factors, and enabling factors were chosen based on empirical support identified in the literature. Physical 
activity self-efficacy has been shown to be the most significant correlate and predictor of physical activity among children (Rimal, 2001; Strauss, Loree, Burak, Rozdilsky, \& Colin, 2000), and physical activity enjoyment is the primary outcome expectancy and intrinsic motivation for children to be active (Kendzierski \& Decarlo, 1991; Motl et al., 2001). Thus, physical activity self-efficacy and physical activity enjoyment were used to address the predisposing factors of physical activity.

For younger children whose behaviors are under less volitional control, parents are the primary providers of supportive opportunities for children to be active (Beets, Cardinal, \& Alderman, 2010). Specifically, parents serve a "gate keeper" role to physical activity through controlling access to physical activity programs, facilities, and outdoor environment (Beets, Cardinal, et al., 2010; Boufous, Finch, \& Bauman, 2004; Welk et al., 2003). Thus, parental influence, such as encouragement and role modeling, was used to address the reinforcing factors of physical activity. Finally, children's physical activity levels are positively correlated with environmental recreational infrastructure, transport infrastructure and safety (Davison \& Lawson, 2006). Environment facilitates opportunities for children to have safe accessible equipment or supplies (toys, balls, and roller skates) to be active (Committee on Environmental Health, 2009; Molnar, Gortmaker, Bull, \& Buka, 2004). So, environment was used to address the enabling factors of physical activity.

Personal demographics - child's age, sex, ethnicity, race, number of siblings or children in household, socioeconomic status, parents' education levels, marital status, and employment status - were collected. This information was collected because children's physical activity levels are significantly correlated with children's age, sex, ethnicity, 
race, sibling's presence, family socio-economic status, parents' marital status, employment status and education levels (Duke \& Huhman, 2003; Hesketh, Crawford, \& Salmon, 2006; McVeigh, Norris, \& de Wet, 2004; Troiano et al., 2008). Thus, these personal demographics were examined to see how these factors influenced other variables, which might provide valuable information for designing tailored physical activity interventions for children with different demographic characteristics. Moreover, children's transportation patterns from home to school were assessed because getting to or from school is the most universal opportunity for incidental physical activity among children (Committee on Environmental Health, 2009). Finally, children's sedentary behavior (screen-viewing time) was assessed to examine its relationship with childhood obesity status.

\section{Research Questions}

1. What is the influence of wearing pedometers on the determinants (physical activity self-efficacy, physical activity enjoyment, parental influence, and environment) of children's physical activity?

2. What are the interrelationships among physical activity self-efficacy, physical activity enjoyment, parental influence, environment, and physical activity among children aged 8 to 11 years old?

3. What are the significant psychosocial and environmental determinants of selfreported physical activity levels among children aged 8 to 11 years old?

4. What are the significant psychosocial and environmental determinants of objective physical activity levels assessed by average pedometer steps among children aged 8 to 11 years old? 


\section{Significance of the Study}

This study points out the need of screening children for obesity annually by school nurses and other primary health care professionals, but also highlights the importance of school nurses or other primary health care professionals to design and conduct tailored effective physical activity education and promotion programs for children in school, family and community settings. It also emphasizes the importance of conducting physical activity programs to prevent or control obesity in childhood based on a theoretical framework that was developed for children and with the collaboration of parents. School nurses play a vital role in promoting a healthy school environment. However, evidence indicates that they lack knowledge related to physical activity and obesity (Story et al., 2002). Thus, this study may provide them necessary knowledge related to the determinants of physical activity and their influencing mechanisms on physical activity.

In this study, physical activity levels were assessed by a self-report scale and pedometers. A pretest-posttest study was also conducted to examine the influence of wearing pedometers on the determinants of physical activity. Therefore, findings from this study will provide valuable information related to the compliance rate, pedometer returning rate, reliability, and reactivity of wearing pedometers in elementary school children. The strategies used in this study to improve the participation rate and compliance rate of wearing pedometers may be helpful for future physical activity studies in children attending afterschool programs.

If this study finds support for the selected factors drawn from the YPAP model, then tailored physical activity interventions for children attending afterschool programs 
with different personal demographics should target physical activity self-efficacy, physical activity enjoyment, parental influence and environment when designing interventions. The statistical method Structural Equation Modeling used in this study will provide information related to mediators between the determinants of physical activity and physical activity. The mediators are essential for designing effective physical activity interventions in elementary school children.

The most frequent barriers for health care professionals to manage childhood obesity are lack of parent involvement, lack of child motivation, and lack of support service (Story et al., 2002). Results from this study will provide health care professionals information related to parental support and other determinants of physical activity. Additionally, the variables proposed in this study can be used by health care professionals to identify the barriers of physical activity for children. Results from this study may also provide evidence for health care professionals and policy makers to advocate new policies to promote physically active lifestyles and prevent/control childhood obesity. Moreover, this study calls attention to new theory testing and modification among children.

\section{Definition of Terms}

The following conceptual definitions were used in this study.

Child and Adolescent: In this study, a child is defined as a person aged 4 to 11 years old, and an adolescent is defined as a person aged 12 to 19 years old (Centers for Disease Control and Prevention [CDC], 2011b). For the purpose of this study, children aged 8 to 11 years old will be recruited. 
Body Mass Index (BMI): Child's BMI is an index calculated from weight and height using the formula ' $B M I=\left(\right.$ Weight in Kilograms $/(\text { Height in Meters })^{2}$ '. For children, BMI is age- and sex-specific, and is often referred as BMI-for-age. BMI cannot directly measure body fat, but it has been shown to be a reliable indicator of body fatness for most children. After obtaining BMI, the number is plotted on a CDC-developed BMIfor-Age chart to obtain a percentile ranking. The BMI percentile is an indicator used to assess the size and growth patterns of individual child in the U.S. (CDC, 2011a). The BMI z-score or standard deviation (SD) score is the number of SD units above or below the mean or median (de Onis et al., 2007).

Overweight: Childhood overweight is defined as a BMI at, or above, the 85 th percentile and lower than the 95th percentile for children of the same age and sex (CDC, 2011e).

Obesity: Childhood obesity is defined as a BMI at or above the 95th percentile for children of the same age and sex (CDC, 2011e).

Physical Education: Physical education is a planned and sequential curriculum program that follows national standards to provide children developmentally appropriate, cognitive content and learning experiences in a variety of physical activity areas (NASPE, 2013). A high quality physical education program should include three components: opportunity to learn, meaningful content and appropriate instruction (NASPE, 2013).

\section{Physical Activity}

Physical Activity: Physical activity refers to "any bodily movement produced by skeletal muscles that results in energy expenditure" (Caspersen, Powell, \& Christenson, 1985, p. 126). 
Exercise: Exercise is physical activity that is planned, structured, repetitive and purposive to improve or maintain some component of physical fitness (Caspersen et al., 1985). Exercise is one subset of physical activity, that is, physical activity includes exercise.

Physical Fitness: Physical fitness is a set of attributes that people have or achieve that relate to the ability to perform movement. Physical fitness has five components: cardiorespiratory fitness, body composition, muscular strength, muscular endurance and flexibility (Caspersen et al., 1985).

Physical Activity Patterns: Patterns of physical activity can be described by type, frequency, duration and intensity.

Type: Physical activity is usually categorized based on the context in which it occurs. Generally, there are four types of physical activity - recreational activity, household activity, occupational activity and transportation activity (Welk, 2002).

Frequency: Frequency refers to the number of activity sessions during a specific time period such as per day, week or month (Vanhees et al., 2005). To assess the frequency of physical activity in children, the number of bouts per day or week, or the percentage of time spent being active on a given day, is often reported. For children, the better criterion for defining frequency may be the number of reporting 2-3 bouts of short and intermittent activity (total 30-60 minutes) per day (Welk, Corbin, \& Dale, 2000).

Duration: Duration is described as the amount of time spent participating in a single bout of physical activity (Vanhees et al., 2005). The duration of 
physical activity is usually reported in minutes of physical activity (Welk et al., 2000).

Intensity: Intensity is defined as individuals' physiological effort when participating in one type of physical activity (Vanhees et al., 2005). The Metabolic Equivalent (MET) is usually used to assess the intensity of physical activity. One MET represents resting energy expenditure of approximate 3.5 $\mathrm{ml} / \mathrm{kg} / \mathrm{min}$ in terms of oxygen consumption. Based on the value of METs in adults, physical activity can be divided into three categories: light-intensity physical activity (1.5-2.9 METs), moderate-intensity physical activity (3.0-6.0 METs), and vigorous-intensity physical activity (> 6.0 METs) (Cliff \& Janssen, 2011). However, the MET values for intensity of physical activity are not well established for children, thus using METs values for adults to assess the intensity of physical activity in children may not be highly accurate (Welk et al., 2000). The U.S. Department of Health and Human Services, the CDC, and the World Health Organization (WHO) use the definition of relative intensity, that is, the child's heart beats faster and he/she breathes harder than normal when performing moderate- or vigorous-intensity physical activity (U.S. Department of Health \& Human Services [USDHHS], 2008).

\section{Physical Activity Determinants Based on the YPAP Model}

Predisposing Factors: Predisposing factors include "knowledge, attitudes, beliefs, values and perceptions that facilitate or hinder motivation for change" (Green \& Kreuter, 2005, p. 14), such as early childhood experiences, attitudes, beliefs, values and perceptions. 
Physical Activity Self-efficacy: Physical activity self-efficacy is defined as individuals' beliefs regarding their own capabilities to participate in physical activity (Bandura, 1997).

Physical Activity Enjoyment: Physical activity enjoyment is defined as "a positive affective state that reflects feelings such as pleasure, liking and fun" when participating in physical activity for children (Motl et al., 2001, p. 110). Reinforcing Factors: Reinforcing factors are the "rewards received and the feedback the learner receives from others following adoption of a behavior, which may encourage or discourage continuation of the behavior" (Green \& Kreuter, 2005, p. 15).

Parental Influence: Parental influence is the perceived impact of parental encouragement, involvement, facilitation and role modeling on children's physical activity participation (Welk et al., 2003). Parental encouragement refers to a parent's verbal or nonverbal encouragement or praise for a child to be active. Parental involvement refers to direct participation of a parent in a child's physical activity. Parental facilitation refers to the assistance parents provide to make it easier for child to be physically active, while parental role modeling refers to parental efforts to model a physically active lifestyle for child. Moreover, parental encouragement, involvement, and facilitation comprise parental support (Welk et al., 2003).

Enabling Factors: Enabling factors are "those skills, resources or barriers that can help or hinder a desired behavioral change as well as environmental change" (Green \& Kreuter, 2005, p. 15), such as facilities and personal or community resources, income or health insurance, laws and statutes, and skills required for a desired behavior. 
Environment: Environment includes two components: equipment accessibility and neighborhood safety (Motl et al., 2005).

\section{Summary}

With the rapidly increasing prevalence of childhood obesity and low physical activity levels among American children, effective physical activity interventions are urgently needed to increase children's physical activity levels and help to control childhood obesity. In order to design effective tailored physical activity interventions, researchers must thoroughly understand the determinants of physical activity in children. To understand the determinants of physical activity in children, population- and behaviorspecific theoretical models that adequately explain the variance of physical activity are necessary. The YPAP model is developed specifically for promoting physical activity among children and adolescents by considering their developmental, psychological, and behavioral characteristics, but this model has not been fully examined in children. Thus, the purpose of this study was to explore the psychosocial and environmental determinants of physical activity among children aged 8 to 11 years old through examining the relationships among physical activity self-efficacy, physical activity enjoyment, parental influence, environment, and physical activity, guided by the population- and behaviorspecific YPAP model. In addition, a pretest-posttest study was conducted to examine the influence of wearing pedometers on the hypothesized determinants of physical activity. Findings from this study may provide valuable information for developing healthy behavior promotion models for children, using pedometers to assess children's physical activity levels, and designing effective physical activity interventions to control childhood obesity. 


\section{CHAPTER II}

\section{BACKGROUND AND SIGNIFICANCE}

This study aimed to explore the psychosocial and environmental determinants of physical activity among children aged 8 to 11 years old. Findings from this study will provide valuable information for effective physical activity interventions designed to control childhood obesity. First, this chapter presents a review of literature related to: (a) elements and patterns of physical activity; (b) physical activity measurements; (c) physical activity in children; (d) correlation between physical activity and childhood obesity; (e) physical activity interventions in children; and (f) determinants of physical activity in children. Then, the conceptual framework is discussed, including the rational for selecting the YPAP model, based on the Precede-Proceed Health Promotion Planning Model, and existing empirical uses of YPAP model.

\section{Part 1: Literature Review}

\section{Elements and Patterns of Physical Activity}

Elements of physical activity. Physical activity is bodily movement of skeletal

muscles that expends energy (Caspersen et al., 1985, p. 126). Generally, physical activity has four elements: (1) bodily movement produced by skeletal muscles, (2) energy expenditure resulting from movement, (3) continuous variations in energy expenditure from low to high, and (4) positive correlation with physical fitness (Caspersen et al., 1985). Based on the above definition, physical activity has two major dimensions - a movement dimension and an energy expenditure dimension. Recently, researchers 
proposed that since physical activity was voluntary, then consideration of physical activity should also involve a behavioral dimension (Freedson \& Miller, 2000). Accordingly, the theoretical domain of physical activity should include behavioral, movement, and energy expenditure dimensions.

To understand physical activity, the differences among physical activity, exercise, and physical fitness need to be explained. Exercise and physical activity have been used interchangeably in the past, but in fact, exercise is a subset of physical activity, that is, exercise is one kind of physical activity (Caspersen et al., 1985). While physical activity is any kind of skeletal muscle movement that burns energy, exercise refers to physical activity that is planned, structured, repetitive, and purposive to improve or maintain some components of physical fitness. In contrast to physical activity and exercise, which refer to the movements people perform, physical fitness is "a set of attributes that people have or achieve" that relate to the physical ability to perform movement (Caspersen et al., 1985, p. 128). Five physical fitness components have been proposed: cardiorespiratory endurance, body composition, muscular strength, muscular endurance, and flexibility. Cardiorespiratory endurance is the ability of circulatory and respiratory systems to supply fuel and oxygen to working muscles during sustained physical activity, and eliminate byproducts of muscle fatigue. Based on the two-compartment model, body composition includes fat and lean mass (Phillips \& Shulman, 2012). Muscle strength is defined as the greatest amount of external force a muscle or muscle group can exert (Caspersen et al., 1985). Muscle endurance refers to the ability of a muscle or muscle group to exert an external force for many repetitions or for an extended period of time. Muscle flexibility is the ability of a joint or joint group to move an entire and normal range of motion. 
Exercise is intended to improve physical fitness rather than to achieve an established level of physical fitness (Caspersen et al., 1985).

Patterns of physical activity. Patterns of physical activity can be described by type, frequency, duration, and intensity. Type of physical activity is usually categorized based on four typical contexts in which physical activity occurs: recreational activity, household activity, occupational activity, and transportation activity (Welk, 2002). Recreational activity refers to activity individuals participate in during free-time, based on personal interests and needs such as walking, hiking, and cycling (Howley, 2001). Household activity is a series of activities that individuals engage in through housework such as laundry, and scrubbing floors (Ridley, Ainsworth, \& Olds, 2008; Wattanasit, 2009). Occupational activity refers to activity related to the performance of a job and usually happens within the period of an eight-hour workday (Howley, 2001). Transportation activity refers to any activity related to active transportation to or from places such as climbing stairs, bicycling to school, and walking to the grocery store (Wattanasit, 2009).

Frequency refers to the number of activity sessions in a specific period of time such as per day, week or month (Vanhees et al., 2005). To assess the frequency of physical activity in children, the number of bouts per day or week or the percentage of time spent being active on a given day is reported. For children, the better criterion for defining frequency may be the percentage of children reporting 2-3 bouts of short and intermittent activity totaling 30-60 minutes on at least 5 days a week (Welk et al., 2000). Duration is described as the amount of time spent participating in a single bout of physical activity (Vanhees et al., 2005). The duration of physical activity is usually 
reported in minutes of time being physically active (Welk et al., 2000). Intensity is defined as the individuals' physiological effort when participating in physical activity (Vanhees et al., 2005). The Metabolic Equivalent (MET) is usually used to assess the intensity of physical activity. One MET represents resting energy expenditure of approximate $3.5 \mathrm{ml} / \mathrm{kg} / \mathrm{min}$ in terms of oxygen consumption. Therefore, physical activity can be quantified in terms of METs, i.e., multiples of resting energy expenditures. However, only $35 \%$ of MET values for intensity of physical activity among children were established based on data measured in children, thus using the current METs values to assess the intensity of physical activity in children may not be highly accurate (Ridley et al., 2008; Welk et al., 2000). Therefore, this study did not assess the intensity of physical activity among children.

In summary, physical activity is any bodily movement produced by skeletal muscles that can result in energy expenditure. Patterns of physical activity can be described by type, frequency, duration, and intensity.

\section{Physical Activity Measurements}

To accurately assess physical activity among children, an instrument must be sensitive enough to identify or record sporadic and intermittent activity. A variety of instruments have been developed to assess physical activity levels among children. These instruments can be organized into two categories based on the type of information they provide: (1) subjective or self-report instruments, and (2) objective instruments (Sirard \& Pate, 2001). Self-report measurements are used most commonly to assess physical activity due to low cost, low burden, less time consumption, and ease of administration among large numbers of children (Sallis, 1991). A number of self-report instruments have 
been developed to assess physical activity levels among children. Physical activity selfreport instruments vary considerably in the specificity with which type, duration, frequency, and intensity of physical activity are evaluated based on the purpose of the study. Generally, self-report measures use recall time frames ranging from as short as one day to as long as one year. Six instruments, the Self Report of Aerobic Activity, the Interviewer-administered Physical Activity Checklists (PACI), the Self-administered Physical Activity Checklists (SAPAC), the Seven-day Physical Activity Recall (PAR), the Physical Activity Questionnaire for Older Children (PAQ-C), and the Fels Physical Activity Questionnaire (PAQ), were identified which assess physical activity levels among children (See Appendix A).

Objective instruments include laboratory methods (direct or indirect calorimetry, and the doubly labeled water method), and mechanical or electronic monitoring (heart rate monitor, pedometer, and accelerometer). Laboratory methods are the most reliable and valid methods to assess physical activity levels, but they are expensive and difficult to implement (Kohl, Fulton, \& Caspersen, 2000). These weaknesses limit their applicability to large-scale epidemiological studies. Laboratory methods can be used to validate other measurements, and are considered the gold standard for assessing physical activity in children (Sirard \& Pate, 2001).

Compared to laboratory methods, electronic monitoring methods are more costeffective for large-scale studies. The validity of heart rate monitors on assessing physical activity relies on the linear relationship between heart rate and oxygen consumption. However, this relationship is not robust, because children's heart rate can also be affected by psychological and environmental stress (Sirard \& Pate, 2001). To avoid the influences 
of psychological and environmental stress on heart rate, researchers need to calibrate individual heart rate/oxygen consumption relationship (Sirard \& Pate, 2001). A mechanical method of objectively monitoring physical activity, pedometers, are typically simple, low cost electronic devices used to estimate the number of steps taken over a period of time. Step counts are a suitable marker of physical activity levels in children, though they do not describe intensity and duration of physical activity (McClain \& Tudor-Locke, 2009). Accelerometers can be used to assess the intensity and duration of physical activity among children, but they are more expensive $(\$ 50-\$ 400)$ than pedometers (\$10-\$50) (McClain \& Tudor-Locke, 2009). Additionally, most accelerometers are not equipped with a digital screen to display the instrument output, thus additional software (price ranges from $\$ 200$ to $\$ 400$ ) is needed to download the data (McClain \& Tudor-Locke, 2009). Therefore, because pedometers are relatively inexpensive, objective, and valid electronic devices, and are considered appropriate instruments to assess objective physical activity levels among children.

Both self-report and objective measurement instruments available to assess physical activity levels among children have been evaluated. Self-report measurements are commonly used to assess physical activity levels due to low cost, low burden, less time consumption, and ease of administration among large numbers of children. The PAQ-C is considered to be the most reliable and valid self-report measurement to assess physical activity levels in children. While there are more accurate objective measures, pedometers are relatively inexpensive, objective, and valid electronic devices to assess objective physical activity levels in children. Therefore, both the self-report instrument 
(PAQ-C) and an objective method (pedometers) were used to assess children's physical activity levels in this study.

\section{Physical Activity in Children}

Recommendations and national objectives. The Centers for Disease Control and Prevention (CDC) (2011c) and the U.S. Department of Health and Human Services (USDHHS) (2009) recommend that children should participate in at least 60 minutes of physical activity per day. Specifically, children should participate in several intermittent bouts of moderate- to vigorous-intensity aerobic physical activity daily, as well as muscle- and bone-strengthening physical activity at least 3 days a week (USDHHS, 2009). Healthy People 2020 defines several national physical activity objectives for children including: (1) increase the proportion of public and private elementary schools that require daily physical education for all students from $3.8 \%$ to $4.2 \%$; (2) increase the proportion of states requiring regularly scheduled elementary school recess from $14.0 \%$ to $34.0 \%$; (3) increase the proportion of children viewing television, videos or playing video games for no more than 2 hours a day from $78.9 \%$ to $86.8 \%$; (4) increase the proportion of physician office visits including physical activity counseling from $7.9 \%$ to $8.7 \%$; and (5) increase the proportion of children's trips to school of 1 mile or less by walking and 2 miles or less by bicycling (USDHHS, 2010). The American Academy of Pediatrics (AAP) recommended limiting children's leisure screen-viewing time, such as TV, movies, video, and computer games, to no more than two hours per day (USDHHS, National Institute of Health [NIH], \& National Heart, Lung, and Blood Institute [NHLBI], 2011) 
Physical activity. For children who participate in physical activity, the most frequent free-time activities are riding bicycles and playing basketball. About $77.4 \%$ of children participate in free-time physical activity, but only $38.5 \%$ of children engage in organized sports (Duke \& Huhman, 2003). The 2003-2004 NHANES showed that on average boys and girls performed 45 and 26 minutes of moderate- or vigorous-intensity physical activity per day respectively, and only $48 \%$ of boys and $35 \%$ of girls participated in at least 60 minutes of physical activity daily (Troiano et al., 2008). The 2009-2010 NHANES data showed that $70 \%$ of children met physical activity recommendation, and 54\% met screen-viewing time recommendation, but only $38 \%$ met both physical activity and screen-viewing time recommendations concurrently (Fakhouri, Hughes, Brody, Kit, \& Ogden, 2013). The 2011 Youth Risk Behavior Survey indicated that only $28.7 \%$ of adolescents participated in at least 60 minutes physical activity daily (USDHHS \& CDC, 2012). Physical activity levels among American children were reported to drop sharply between age 9 and age 15, and the majority of children did not meet the daily physical activity recommendation (Bock \& Miller, 2008). At age 9, children performed, on average, three hours of moderate- or vigorous-intensity physical activity per day, but by age 15 , they performed only an average of 49 minutes per weekday and 35 minutes per weekend (Bock \& Miller, 2008).

Sedentary activity. In 2002, the Youth Media Campaign indicated that $61.5 \%$ of children did not engage in any organized physical activity during non-school hours and $22.6 \%$ of children did not participate in any free-time physical activity (Duke \& Huhman, 2003). The 2001-2004 NHANES data indicated that boys and girls aged 9 to 11 years old played an average of 144 minutes of computer/video games per day, and watched about 
72 minutes and 48 minutes of TV respectively per day (Anderson, Economos, \& Must, 2008). Another study found that boys and girls watched a daily average of 137 and 128 minutes of TV respectively, and approximately $30 \%$ of boys and $25 \%$ of girls watched four hours or more TV per day (Marshall, Gorely, \& Biddle, 2006). On average, boys played about 60 minutes of video games per day compared to 23 minutes for girls, and children used computers for about 30 minutes per day (Marshall et al., 2006). More than two thirds of children had a TV in their bedroom, and about $40 \%$ of families had a TV in a room where meals were eaten (Jordan \& Robinson, 2008). Screen-viewing time (watching TV, using computers, or playing video games) increased as children got older (Fakhouri et al., 2013).

Physical education. Physical education is a planned and sequential curriculum program that follows national standards to provide children developmentally appropriate, cognitive content and learning experiences in a variety of physical activity areas (NASPE, 2013). More than $69 \%$ of elementary schools, $84 \%$ of middle schools, and $95 \%$ of high schools were recommended by the National Association for Sport and Physical Education (NASPE) to have physical education. However, only $4 \%$ of elementary schools, $8 \%$ of middle schools, and $3 \%$ of high schools had provided daily physical education to students (Lee et al., 2007). The NASPE (2010) recommended that schools should provide 150 minutes of instructional physical education per week for elementary school children, and 225 minutes for middle and high school students throughout the school year. However, only three states in America required the nationally recommended 150 or 225 minutes physical education per week (NASPE, 2010). 
Physical activity and demographics. Children's physical activity levels are significantly correlated with ethnicity, race, sex, presence of siblings, family income, parents' education levels, marital status, and parental employment status. Generally, boys participated in higher levels of physical activity than girls (Crespo et al., 2001). Hispanic children with parents who have low income and education levels engage in lower levels of organized activity and perceive more barriers, such as transportation, expense, and neighborhood safety, than other groups of children (Duke \& Huhman, 2003). Children from households with two parents participate in higher levels of physical activity compared to children from single-parent households (McVeigh et al., 2004). One study among 2,458 children indicated that parental employment levels were also negatively correlated with children's television viewing, and children with employed mothers tended to engage in more moderate- to vigorous-intensity physical activity (Hesketh et al., 2006). Moreover, children from single-parent households, children with fathers not in paid employment, and those without siblings participate in more low-intensity physical activity, while children in similar households, but with siblings, engage in more moderate- to vigorous-intensity physical activity (Hesketh et al., 2006).

In summary, the majority of American children do not meet daily physical activity recommendations, and only three states require the nationally recommend standards for physical education. On average, children spend more time watching TV than participating in physical activity. Children's physical activity levels are significantly correlated with their ethnicity, race, sex, presence of siblings, annual family income, parents' education levels, marital status, and employment. 


\section{Correlation between Physical Activity and Childhood Obesity}

Many efforts have been made to investigate factors contributing to childhood obesity in order to promote children's health and reduce costs of obesity-related health problems. Lack of physical activity or sedentary lifestyle is a significant determinant of childhood obesity, and physical activity has been reported to be the key to controlling childhood obesity (Hills et al., 2011; Janssen et al., 2005). Physical activity plays an important role in reducing body fat mass through increasing energy expenditure (Nowicka \& Flodmark, 2007). Weight is gained when energy intake exceeds energy expenditure, and weight is lost when energy expenditure exceeds intake. Energy expenditure is comprised of three components: resting energy expenditure $(60-75 \%)$, the thermic effects of food (the energy expenditure in consuming and processing food, 10\%), and the thermic effects of physical activity (15-30\%). Among the three components of energy expenditure, thermic effects of physical activity are the most variable component, varying within and between individuals due to the mutable nature of physical activity (Goran \& Treuth, 2001). Thus, physical activity is the discretionary component of energy expenditure.

Consistent evidence shows that overweight or obese children have lower physical activity levels and poorer fundamental movement skills compared to non-overweight children (Hills et al., 2011; Nowicka \& Flodmark, 2007; Steinbeck, 2001). Furthermore, children with higher levels of physical activity have reduced skinfold thickness (Davis et al., 2007; Hills, King, \& Armstrong, 2007; Nowicka \& Flodmark, 2007). One literature review of physical activity intervention studies supported the inverse relationship of physical activity levels with the prevalence of childhood obesity, and it concluded that 
increasing physical activity levels should be an attractive and non-restrictive approach to childhood obesity intervention (Steinbeck, 2001). Similarly, the 2001-2002 Health Behavior Survey among 137,593 elementary school-aged children from 34 countries found that greater physical activity participation was significantly correlated with lower odds of overweight in 29 out of 34 countries including U.S. (Janssen et al., 2005). The Health Behavior Survey concluded that increasing physical activity participation should be the focus of strategies aimed at controlling childhood obesity.

A recent review of 16 new cohort studies among children found that 6 out of 11 studies reported that children's weight gain was negatively related to self-reported physical activity levels but positively related to sedentary activities such as watching TV or playing video games. Two out of five studies using objective measurements to assess physical activity levels reported significantly negative relationship between physical activity and obesity (Wareham, van Sluijs, \& Ekelund, 2005). Another study among 1,204 children and adolescents showed that lack of physical activity and sedentary lifestyles were significant determinants of elevated BMI, and risk factors contributing to overweight and obesity development in youth (Bukara-Radujkovic \& Zdravkovic, 2009). Additionally, pediatric experts indicated that the evidence of a negative correlation between physical activity and obesity in children was very strong (Davis et al., 2007).

Physical activity and health. In addition to helping control childhood obesity, regular physical activity has a number of other desirable influences on children's normal growth, health, and academic performance. Evidence has indicated that physical activity is essential for adequate childhood growth and development of cardiorespiratory endurance, muscle strength, flexibility, motor skills, and agility (Hills, Okely, \& Baur, 
2010; Nowicka \& Flodmark, 2007). Lack of regular physical activity is correlated with cardiovascular risk factors such as blood lipids and fasting glycemia (Nowicka \& Flodmark, 2007). Regular physical activity can significantly promote cardiovascular health through improving lipid profiles, and decreasing the incidence of hypertension and metabolic syndrome (Strong et al., 2005). In addition, evidence shows that regular physical activity can decrease anxiety and depression, and improve self-concept among children (Strong et al., 2005). More time spent on physical activity does not negatively influence children's academic performance. On the contrary, it can improve students' academic achievement through enhancement of concentration and improved classroom behaviors (Smith \& Lounsbery, 2009).

In summary, overweight or obese children engage in lower levels of physical activity compared to non-overweight children, suggesting that lack of physical activity is one significant contributor to the childhood obesity epidemic. Moreover, physical activity has beneficial effects on children's growth, health, and cognition. Therefore, improving children's physical activity levels not only can help control childhood obesity, but also can promote children's normal growth, physical health, and academic performance.

\section{Physical Activity Interventions in Children}

With the strong evidence supporting a negative relationship between physical activity and childhood obesity, many physical activity interventions have been designed to increase children's physical activity levels and control childhood obesity. However, the majority of physical activity interventions, including school-based programs, remain largely ineffective in childhood obesity control. A meta-analysis of 18 studies reported that school-based physical activity interventions, with durations ranging from 6 months to 
3 years, had no significant effect on decreasing BMI (Harris et al., 2009). Similarly, Dobbins and colleagues (2009) did a review of 26 studies on school-based physical activity interventions and concluded that physical activity interventions did not significantly affect BMI, pulse rate, leisure time physical activity rate, and systolic or diastolic blood pressure. Another review of 11 clinical trials, intended to reduce weight gain by increasing physical activity or reducing sedentary behavior among children, found that only three trials reported a small intervention effect on obesity indices (BMI and skinfold thickness), and two of those three studies found significant effect only in boys (Wareham et al., 2005). Additionally, Brown and Summerbell (2009) did a systematic review of school-based interventions, and found that only five out of 15 physical activity interventions demonstrated significant BMI decrease, and two of the five studies found BMI decrease only in girls.

Some researchers attribute the ineffectiveness of physical activity interventions to an insufficient dose of physical activity, due to short duration of intervention, insufficient quantity of physical activity, or low adherence to intervention protocols (Dobbins et al., 2009; Harris et al., 2009). Beyond the dose of physical activity, the effectiveness of physical activity interventions may depend on the measurements of physical activity (self-report measurements vs. objective measurements), study design (not randomized controlled design), intervention methods (not focused on determinants of physical activity or not based on a theoretical framework) and evaluation methods (measure the composition of the weight loss vs. BMI) (Steinbeck, 2001). Based on the twocompartment model, the body is divided into body fat mass and lean mass (Phillips \& 
Shulman, 2012). Both body fat mass and lean mass increase with age throughout childhood, and vary based on sex and ethnicity (Phillips \& Shulman, 2012).

Measurements used to assess body composition can be divided into two categories: direct measures and indirect measures. Direct measures, such as densitometry (underwater weighing) and scanning using dual energy $\mathrm{x}$-ray absorptiometry, are more accurate than indirect measures but also more expensive, time consuming, and not as practical, especially for large epidemiological studies (Kipping, Jago, \& Lawlor, 2008). Indirect measures, such as waist circumference, skin fold, and BMI, do not precisely assess children's body composition (percent body fat), and there is considerable variation across age, sex, and ethnicity (Dehghan \& Merchant, 2008). But while BMI does not precisely assess body composition, it has been recommended as a measure to screen for childhood obesity in clinical practice and epidemiology because, in addition to its low cost and ease of use, it has high specificity (95\%-100\%) and moderate sensitivity (36\%66\%) (Moyer et al., 2005).

Though physical activity interventions may not have significant effects on childhood weight control, they do show encouraging effects on increasing physical activity levels, decreasing sedentary activities, and preventing an increase in prevalence of childhood obesity (Dobbins et al., 2009). A randomized controlled study in 24 elementary schools over 3 years found that though the Physical Activity Across the Curriculum (PAAC) program, focusing on increasing physical activity without reducing learning time, did not have significant effects on weight control (BMI), it did show that change in BMI was significantly influenced by exposure to the program with increased minutes of exposure in the program indicating decreased BMI change. In addition, 
schools with the PAAC program did show significantly less increase in BMI, higher increase in physical activity, and higher academic achievement scores than control schools (Donnelly et al., 2009). One comprehensive review found that physical activity programs of 30- to 60 -minute duration, performed 3 to 7 days per week, demonstrated significant body fat and visceral adiposity reduction among obese children and adolescents but not among healthy weight children and adolescents (Strong et al., 2005). This finding implies that physical activity interventions may be more effective in reducing weight among obese children.

The AAP recommends promoting lifelong habits of physical activity to achieve sustained weight loss rather than short bouts of exercise (Council on Sports Medicine and Fitness \& Council on School Health, 2006). Though physical activity interventions of short duration have not shown to significantly decrease BMI, they are effective in increasing tolerance of physical activity, reducing blood cholesterol, time in watching television, and improving physical fitness levels (Dobbins et al., 2009). Thus, physical activity interventions should first focus on helping children establish and maintain physically active lifestyles throughout childhood. Theoretically, early physical activity interventions should be more effective in controlling childhood obesity because children learn lifestyle behaviors from an early age and are more flexible in their abilities to change behaviors than adults, but no studies have investigated this hypothesis (Steinbeck, 2001).

Evidence does indicate that understanding factors contributing to physical activity in children will help build a framework for prevention or intervention (Steinbeck, 2001). Thus, in order to design effective physical activity interventions tailored to address the 
objectives of Healthy People 2020 - reduce childhood obesity from $17.4 \%$ to $15.7 \%$ and prevent inappropriate weight gain (USDHHS, 2010), a theoretical framework that considers the determinants of physical activity and has been validated in children is necessary to guide further intervention design.

In summary, considering the increasing trend of childhood obesity prevalence and the promising role of physical activity in controlling childhood obesity, many physical activity interventions have been designed and conducted to control the childhood obesity epidemic. However, the majority of physical activity interventions remain largely ineffective in reducing BMI or other obesity indices. To design effective physical activity interventions among children, it is important for interventions to be based on a theoretical model that adequately explains the variance of physical activity. Thus, a theoretical framework of the determinants of physical activity in children is needed to guide future intervention study designs.

\section{Determinants of Physical Activity in Children}

The determinants of physical activity in children are multifactorial, resulting from interactions among a number of factors. A discussion of the variables used in this study (physical activity self-efficacy, physical activity enjoyment, parental influence, and environment) will help to clarify the relationships among the factors that affect physical activity in children and those considered to be the determinants of physical activity.

Physical activity self-efficacy. Self-efficacy was originally defined as the selfjudged conviction that one can successfully execute the behavior required to produce an outcome (Bandura, 1977). Bandura (1997) later refined the definition of self-efficacy to encompass individuals' beliefs regarding their own capabilities to perform a task that is 
likely to result in the anticipated outcome. Self-efficacy has been shown to be a significant predictor of physical activity among children (Baranowski, Cullen, Nicklas, Thompson, \& Baranowski, 2003), and evidence on the positive correlation between selfefficacy and physical activity is consistent and strong.

Self-efficacy is positively correlated with physical activity. Scientific findings indicate that obese children usually have lower physical activity self-efficacy than nonobese children (Trost, Kerr, Ward, \& Pate, 2001). In a review of the correlates of physical activity among children and adolescents, half of the studies demonstrated that selfefficacy was positively associated with physical activity (Sallis, Prochaska, \& Taylor, 2000). Dzewaltowski and colleagues (2010) reported that children with higher levels of after-school physical activity had higher physical activity self-efficacy than children with lower levels of physical activity. In a study of 4,779 adolescents, physical activity selfefficacy was positively correlated with physical activity levels, and the differences in physical activity related to sex were explained by the differences in self-efficacy between males and females (Spence et al., 2010). Another study in 72 adolescents found that only self-efficacy made a significant and unique contribution to objective physical activity levels compared to other social cognitive variables such as intention and perceived behavior control (Roberts, Maddison, Magnusson, \& Prapavessis, 2010).

Self-efficacy can facilitate physical activity interventions. A study in 206 African American school-aged children found that an increase in physical activity self-efficacy was significantly correlated with improvement in physical activity (Annesi, Faigenbaum, \& Westcott, 2010). Similarly, a randomized controlled trial among 2,087 adolescent girls in 24 high schools indicated that self-efficacy significantly mediated the effects of 
intervention on physical activity (Dishman et al., 2004). This study concluded that selfefficacy should be a targeted mediator variable in physical activity intervention designs. In reviewing the physical activity interventions in healthy adults, the study found that when an intervention was associated with a change in self-efficacy, the intervention also tended to be associated with a change in physical activity (Williams \& French, 2011).

In conclusion, physical activity self-efficacy is one significant determinant of physical activity. Interventions among adults and adolescents focusing on increasing physical activity self-efficacy have shown significantly increased physical activity levels, but only a few studies have been found that focus on self-efficacy as a mechanism to improve physical activity in children.

Physical activity enjoyment. Enjoyment can be defined as "a positive affective state that reflects feelings such as pleasure, liking and fun" (Motl et al., 2001, p. 110). Recently, physical activity enjoyment and attraction to physical activity have been used interchangeably (King, 2008). In fact, they are different terms. Attraction to physical activity is defined as "an individual's desire to participate in a task involving physical exertion or movement through play, games or sport" (Rose, Larkin, Hands, Howard, \& Parker, 2009, p. 573). Children are attracted to physical activity by physical activity enjoyment, and attraction of physical activity provides a basis for physical activity enjoyment (Rose et al., 2009). Physical activity enjoyment has been reported to be the primary motivation for children to participate in physical activity (DiLorenzo, StuckyRopp, Vander Wal, \& Gotham, 1998; Sallis, Prochaska, Taylor, Hill, \& Geraci, 1999). The Self-Determination Theory (SDT) also supports that enjoyment/pleasure is the intrinsic motivation for individuals to engage in a behavior (Lox et al., 2006; Ryan \& 
Deci, 2000). The SDT was developed by Ryan and Deci (2000) to indicate the importance of human's inner resources for personality development and behavioral selfregulation. A stepwise regression analysis indicated that physical activity enjoyment was the only consistent predictor of physical activity among children (DiLorenzo et al., 1998). Additionally, Sallis, McKenzie and colleagues (1999) found that enjoyment was strongly and consistently correlated with physical activity among children enrolled in the Sports, Play, and Active Recreation for Kids (SPARK) physical education program. The SPARK physical education curriculum is recognized as an effective school-based response to the nations' health care crisis, a successful model to deal with the childhood obesity epidemic, and an Exemplary Program of the U.S. Department of Education (SPARK, 2012). Sallis, McKenzie and colleagues argued that enjoyment should be targeted as an important factor when planning future physical activity intervention programs.

Physical activity enjoyment has been reported to be positively correlated with task goal orientation, perception of athletic competence, perceived physical appearance and self-reported physical activity levels among children (Moore et al., 2009). Moreover, Motl and colleagues (2001) suggested that interventions should focus on enjoyment to improve physical activity among adolescents. A randomized controlled trial to explore the effects of the Life-style Education for Activity Program (LEAP) on physical activity among 2,087 adolescent girls found that physical activity enjoyment had a moderate effect on self-efficacy but only a weak effect on physical activity, suggesting that physical activity enjoyment had an indirect effect on physical activity through the mediator of self-efficacy (Dishman et al., 2005). 
In summary, physical activity enjoyment is a consistent and promising determinant of physical activity in children, and further studies are needed to target physical activity enjoyment as an important consideration when designing physical activity intervention programs for children. More studies are necessary to explore the relationships among physical activity enjoyment, physical activity self-efficacy and physical activity, especially to clarify the mediator or moderator effects of self-efficacy among children.

Parental influence. Social ecological models indicate that family is the most influential microsystem for children (McLeroy, Bibeau, Steckler, \& Glanz, 1988), thus, parental influence should be an important factor influencing children's physical activity. Moreover, for younger children whose behaviors are under less volitional control, parents are the primary providers of supportive opportunities for children to be physically active (Beets, Cardinal, et al., 2010).

According to Welk and colleagues (2003), parental influence can be divided into four components: parental encouragement, parental involvement, parental facilitation, and parental role modeling. Parental encouragement, involvement and facilitation comprise parental support. Parental encouragement refers to parents' verbal or nonverbal encouragement or praise for a child to be active. Parental involvement refers to direct participation of a parent in a child's physical activity. Parental facilitation refers to the assistance parents provide to make it easier for a child to be physically active. Finally, parental role modeling refers to parental efforts to model a physically active lifestyle for a child (Welk et al., 2003). 
Parental influence has been identified as a significant factor in increasing physical activity levels among children (Van der Horst, Paw, Twisk, \& Van Mechelen, 2007). One study among 994 children found that parental influence was positively correlated with children's physical activity, with the parental facilitation component having the greatest influence (Welk et al., 2003). Similarly, a study in 872 Chinese children and adolescents reported that parental influence had direct positive effects on children's physical activity participation (Cheung-Peggy \& Chow, 2010). Another study in 3,114 children found that children's perception of parental support and parents' self-reported support for physical activity were strongly correlated with children's organized physical activity (Heitzler, Martin, Duke, \& Huhman, 2006). In addition, one study found that the parental role modeling component was positively correlated with children's physical activity levels (Katherine, 2009), although another study found that only the mother's role modeling significantly affected children's physical activity (Bois, Sarrazin, Brustad, Trouilloud, \& Cury, 2005). Nine year-old girls' parental support levels significantly predict physical activity levels at age 11 (Davison, Downs, \& Birch, 2006). Similarly, parental encouragement and involvement components significantly predict children's physical activity levels (Smith \& Burton, 2005).

Beyond the direct effects of parental influence on children's physical activity, parental influence also has indirect effects on children's physical activity. Evidence indicates that parental influence could affect children's physical activity through attraction to physical activity and perceived competence (Smith \& Burton, 2005). The parental encouragement component was shown to enhance self-esteem, reduce anxiety, and give obese children a sense of comfort, acceptance and reassurance of self-worth 
(Cox, Martin-Ginis, \& Petruzzello, 2006). In other words, parental influence can improve physical activity indirectly, through its influences on psychological factors, which support the indirect effects of parental influence on physical activity among children.

In summary, parental influence is a significant determinant and predictor of physical activity among children. Evidence indicates that physical activity interventions including contact with families are most effective in increasing children's physical activity levels (Timperio, Salmon, \& Ball, 2004). Thus, families should be an essential part of a physical activity intervention program. The National Association for Sports and Physical Education (NASPE) (2012) recommends that a Comprehensive School Physical Activity Program (CSPAP) should involve parents because parents are an integral part of children's lives and can impact their physical activity participation.

Environment. Sallis and colleagues developed a preliminary social ecological model for environmental and policy interventions to promote physical activity, which focuses primarily on exosystem and macrosystem (Sallis, Bauman, \& Pratt, 1998). According to this model, more supportive environments will lead to higher physical activity participation. Moreover, an environment promoting active lifestyle is a very important factor to enable children to achieve daily physical activity recommendations. It should be noted, however, that social ecological approaches are not intended to be "stand-alone" interventions, with the idea that "if we build it, they will come." In other words, building fitness facilities does not guarantee that people will use the facilities and become physically active (Lox et al., 2006). However, the fitness facilities at least offer the opportunities for children to be physically active. 
Evidence has supported that equipment accessibility (walkable neighborhood, sidewalks or school grounds designed for a variety of physical activity opportunities) can facilitate children's physical activity and improve physical activity levels (Sallis, 2010). Children's physical activity levels increase as the percentage of park area increases within a neighborhood (Roemmich, Epstein, Raja, \& Yin, 2007). Moreover, getting to or from school is the most universal opportunity for incidental physical activity among children. Though walking or bicycling to school has not been shown to decrease BMI or control childhood obesity, it is a valuable opportunity for children to be active and can improve children's physical activity levels (Committee on Environmental Health, 2009). Some researchers found that physical activity self-efficacy mediated the effects of equipment accessibility on physical activity among children (Motl et al., 2005).

In addition to access to equipment, neighborhood safety is a very important determinant of physical activity. Evidence indicates that traffic danger is the most commonly mentioned factor that prevents children from walking to school or participating in physical activity (CDC, 2005). Neighborhood safety plays an important role in parents' and children's responses to the environment. Even though children have access to physical activity equipment or supplies, safety concerns may prevent them from using the accessible playground, parks or gyms (Committee on Environmental Heath, 2009). Children whose parents report anxiety about neighborhood safety engage in lower levels of physical activity (Weir, Etelson, \& Brand, 2006), particularly among urban children and adolescents (Molnar et al., 2004).

In summary, equipment accessibility plays an important role in providing opportunities for children to be active, while neighborhood safety facilitates children's 
use of accessible equipment or supplies. Though environmental change may take a long time and a great deal of money to implement, and require policy support to achieve, environment is a very important determinant for children to participate in physical activity. This is especially important for children with low socioeconomic status, due to limited access to remote or more expensive opportunities for physical activity (Humbert et al., 2006).

Summary. Determinants of physical activity in children can be summarized into two categories: psychosocial (physical activity self-efficacy, physical activity enjoyment, and parental influence) and environmental (environment). Based on the Social Cognitive Theory, self-efficacy and outcome expectancy (enjoyment) are the two primary individual factors influencing physical activity participation (Bandura, 1986). Interventions focusing on increasing physical activity self-efficacy have shown increased physical activity levels, but only a few studies have been found to target self-efficacy as a mechanism to improve physical activity among children. Physical activity enjoyment is a consistent and promising determinant of physical activity among children, but no study has been found that specifically targets enjoyment as a mechanism to improve physical activity among children.

Based on social ecological models, family is the primary microsystem of a child, implying that parental influence plays an important role in influencing children's physical activity. Thus, physical activity interventions should target parental influence to increase children's physical activity. Moreover, equipment accessibility plays an important role in providing opportunities for children to be physically active, while neighborhood safety facilitates children's use of accessible equipment or supplies. Though environment has 
been supported to be significantly correlated with physical activity among children, few studies have targeted environment to improve children's physical activity due to its high cost and great time consumption.

\section{Part 2: Conceptual Framework}

\section{Rational for Choosing YPAP Model}

To explain the rational for choosing the Youth Physical Activity Promotion (YPAP) model for the dissertation study, theories and models popularly used in physical activity studies among children and adolescents will be reviewed and discussed. All the theories and models used in physical activity studies can be summarized into three categories: models of individual health behavior such as Theory of Planned Behavior and Self-Determination Theory, models of interpersonal health behavior such as Social Cognitive Theory, and community and group models of health behavior change such as social ecological models.

\section{Models of individual health behavior.}

Theory of Planned Behavior. The Theory of Planned Behavior (TPB) proposes that people are more likely to perform a behavior when they consciously intend to perform that behavior (Ajzen, 1991). Intention mediates the influences of three principle constructs (attitude towards behavior, subjective norms, and perceived behavioral control) on behaviors. Attitude simply refers to a person's positive or negative thoughts concerning the performance of a behavior. Subjective norms refer to the perceived social pressure of performing a behavior. Perceived behavior control is the degree of control the individual perceives having over a behavior, which can influence behavior independently as well as through the effects of intention (Ajzen, 1991). 
Though the TPB has been popularly used in physical activity studies, only a few studies have been conducted among children. The moderately strong relationships between the three principle constructs and intention are supported by most of these studies, but only a very weak correlation between intention and physical activity is found. A study among 475 children found that intention only accounted for $8-9 \%$ of variance in self-reported moderate- to vigorous-intensity physical activity (Martin, Oliver, \& McCaughtry, 2007). Rhodes and colleagues (2006) found that only $13 \%$ of children who perceived very high intention to be physically active reported actually engaging in daily physical activity, and intention had a very small effect on changes in physical activity over time.

No study among children has been found that explored the reason for the gap between intention and physical activity or why intention can only explain a small amount of the variance in physical activity. Based on the findings of studies among adolescents, the gap between intention and physical activity may be due to individuals who hold an intention to act but subsequently fail to enact that intention (Godin \& Conner, 2008). Self-efficacy is the most common moderator in the relationship between intention and physical activity among adolescents and adults in all domains of behaviors (Dishman et al., 2002a; Luszczynska, Schwarzer, Lippke, \& Mazurkiewicz, 2011). One study of 1,797 adolescents reported that self-efficacy significantly explained $74 \%$ of the variance in intention to be physically active (Dishman et al., 2002b). Moreover, Luszczynska and colleagues (2011) conducted two cluster randomized experimental studies among 58 diabetes patients and 187 active individuals, and their results also supported the moderator role of self-efficacy in the intention-physical activity relationship. 
In summary, the correlation between intention and physical activity proposed by the TPB is weak among children. This theory emphasizes the influence of individuals and their beliefs on physical activity but does not specify particular beliefs about behavioral outcomes, normative referents or control beliefs that should be measured or targeted (Glanz et al., 2008). This results in difficulty applying the theory to children whose behaviors may be influenced in different ways than adolescents or adults.

Self-Determination Theory. The Self-Determination Theory (SDT) was developed by Ryan and Deci (2000) to emphasize the influences of intrinsic interest and extrinsic rewards on behavioral changes. This theory assumes that people have three basic innate needs: (1) a need for self-determination or autonomy, that is, the desire for self-initiation in the regulation of behaviors, (2) a need to demonstrate competence, that is, the desire to effectively interact with the environment and accrue expected outcomes, and (3) a need for relatedness, that is, the desire to be connected and accepted by significant individuals (Ryan \& Deci, 2000). These three innate needs are the facilitators of intrinsic motivation. The theory specifies three forms of motivation: intrinsic motivation, extrinsic motivation, and amotivation. Intrinsic motivation refers to the mentality of performing a behavior for the pure sake of enjoyment, pleasure and satisfaction, and it is the most self-determined type of motivation. Intrinsic motivation is the ideal condition for the promotion of healthy behaviors. Extrinsic motivation involves four regulatory styles: (1) integrated regulation (degree of self-regulation = very high), (2) identified regulation (degree of regulation = moderately high), (3) introjected regulation $($ degree of self-regulation $=$ moderately low $)$, and $(4)$ external regulation $($ degree of selfregulation $=$ very low $($ Ryan \& Deci, 2000). Integrated regulation occurs when 
behavioral goals and actions are congruent with one's values and needs. Identified regulation occurs when a behavior is motivated by personal goals. Introjected regulation occurs when a behavior is performed for the avoidance of guilt and anxiety or the enhancement of pride and ego. Lastly, external regulation occurs when a behavior is performed totally for obtaining a reward or avoiding a punishment. Amotivation involves a lack of intention and absence of motivation, and it is the least autonomous regulation. Self-determination decreases along the continuum from intrinsic motivation to amotivation (Ryan \& Deci, 2000).

Several studies among children and adolescents have supported the hypotheses proposed in the SDT. One study among 239 children and adolescents found that students with higher satisfaction of the three basic innate needs (autonomy, competence and relatedness) had higher intrinsic motivation to perform physical activity, and intrinsic motivation significantly and positively predicted physical activity levels and positive attitudes towards physical activity (Vierling, Standage, \& Treasure, 2007). Similarly, another study in 328 adolescents indicated that the three basic innate needs were positively correlated with intrinsic and extrinsic motivation, and intrinsic and extrinsic motivation were positive predictors of intention to take part in leisure-time physical activity while amotivation was a negative predictor (Standage, Duda, \& Ntoumanis, 2003). Moreover, Standage and colleagues (2005) conducted a study among 950 adolescents that indicated that the three basic innate needs were the facilitators of intrinsic motivation to attend school physical education classes.

There is emerging, but still limited, support for applying the SDT to understanding determinants of physical activity among children (Motl, 2007). Though 
this theory emphasizes the important influences of motivation on physical activity, it ignores the influences of environment on individuals' behaviors. Several studies among adolescents have supported the propositions of this theory, but no study has been found that tested this theory specifically among children. Children under 11 years old have not developed adult-capacity for abstract and logical thought and reason (Piaget, 1952), so they may be motivated in different ways than adolescents or adults.

Limitations. Models of individual health behavior only focus on the influences of individuals and their beliefs on behaviors. Humans do not operate as an autonomous agent, that is, human's behaviors are not only influenced by individuals and their beliefs (Bandura, 1977). Instead, behaviors are strongly affected by the social context in which they occur. The TPB and the SDT do not give sufficient attention to the maintenance of behavioral change, which should be the focus and purpose for behavioral studies (Glanz et al., 2008). For children, whose behaviors are under less volitional control, and who do not fully develop adult-capacity for abstract and logical thought and reason, environment plays an important role in behavioral change.

\section{Model of interpersonal health behavior.}

Social Cognitive Theory. The Social Cognitive Theory (SCT) provides a comprehensive framework for understanding determinants of health-related behaviors (Baranowski et al., 2003). The SCT is based on four assumptions: (1) behavior is purposeful or goal directed; (2) humans are self-reflective agents; (3) humans are capable of self-regulation; and (4) reciprocal determination means that personal, environmental, and behavioral factors interact reciprocally. This theory proposes that behavior is a function of constant reciprocal interaction between aspects of the environment and of the 
person. The primary personal concepts for understanding behavior include skills (the ability to perform behavior when desired), self-efficacy (the belief that one is capable of performing a behavior or action), and outcome expectancies (the belief that a behavior or action will lead to an anticipated outcome) (Bandura, 1986). The key environmental variables include modeling (learning a behavior by watching others and receiving reinforcement for the behavior), and availability (whether physical equipment is available for use) (Bandura, 1986). The primary resources outlined in the SCT are skills and selfefficacy to perform behaviors. Because skills are difficult to measure, thereby difficult to study, self-efficacy has become the focus of behavior change studies (Baranowski et al., 2003). The primary motivational variable in the SCT is outcome expectancies, and enjoyment can be considered as an outcome expectancy for physical activity (Bandura, 1986; Baranowski et al., 2003).

The SCT has been popularly used in research among children to understand the determinants of physical activity. Scientific data shows that environmental factors (physical environment such as access to exercise facilities and community safety, and social environment such as social support from parents), and personal factors (selfefficacy, outcome expectancy, and enjoyment) are the significant determinants of physical activity among children (Sallis et al., 2000).

Limitations. This theory recognizes the influences of environment on behaviors, but it also focuses on human's potential abilities to change and construct environment to meet their needs (Glanz et al., 2008). However, for children who have little power to control their lives, their behaviors are more passively affected by the environment. In addition, this theory can describe determinants of behavior only when the behavior is 
challenging or novel (Lox et al., 2006). As a result, the influence of self-efficacy is greatly reduced as behavior becomes well learned and habitual. The SCT provides a broad and frequently cited source of concepts and principles of behavioral change, but it does not focus on the specific behavior that is being studied - physical activity. Furthermore, this theory focuses on individual behavior change instead of behavioral change at the level of population. It has been recommended that this theory should be merged with other theories composed of similar or shared elements to further enhance its predictive ability (Lox et al., 2006).

\section{Community and group models of health behavior change.}

Social Ecological Models. Social ecological models recognize that individuals are responsible for engaging in healthy behaviors; they also recognize the influences of physical environment, society, community, and government on healthy behaviors (Green, Richard, \& Potvin, 1996). These models have four core principles: (1) human behaviors are influenced by multiple levels of factors including intrapersonal (biological, psychological), interpersonal (social, cultural), organizational, community, physical environment, and policy; (2) these multiple levels of factors interact to influence behaviors; (3) these models should be behavior-specific and identify the most important influences at each level; and (4) interventions targeting multiple-level factors should be more effective in changing behavior (Glanz et al., 2008). Social ecological models propose that behavior change is maximized when individuals are motivated and educated, when social support is present, and when environment and policies support behavioral change (Glanz et al., 2008). 
Ecological Model for Physical Activity. In 2006, Sallis and colleagues developed a social ecological model built around four domains of physical activity: recreational activity, transportation activity, occupational activity, and household activity. This model proposes that the four domains of physical activity take place in different settings and are expected to be affected by different sets of environmental factors. Moreover, each environmental factor is influenced by policies emanating from multiple government agencies, organizations, and society. Intrapersonal variables (demographics, biological, psychological and family situation), and individual perceptions of environment (safety, attractiveness, comfort, perceived crime, convenience and accessibility) are individuallevel factors, while behavior setting (places where physical activity may occur), and policy environment are the environmental-level factors. Behavior represents the interaction of individual-level factors and environmental-level factors. Environment (perceived environment, behavior setting, and policy environment) can be classified within four categories: built environment, natural environment, social-cultural environment, and information environment (Sallis et al., 2006). Built environment is a part of physical environment constructed by human activity such as land use patterns, transportation system, and physical infrastructure of roads, sidewalk and bike paths (Handy, Boarnet, Ewing, \& Killingsworth, 2002).

Evidence supports the idea that built environment (walkable neighborhoods, sidewalks, or school grounds designed for a variety of physical activity opportunities) facilitates children's physical activity and improves physical activity levels (Sallis, 2010). The ecological model for physical activity focuses on multiple levels of influence that broaden options for physical activity interventions. Moreover, policy and environmental 
changes considered in this model affect an entire population, in contrast to interventions that reach only individuals who choose to participate (Glanz et al., 2008). In addition, policy and environmental interventions can establish settings and incentives that persist in sustaining behavioral change over time. Evidence supports that different levels of policy (federal, state, community, and school) can simultaneously and independently influence environment and opportunities for children to be physically active (Langille \& Rodgers, 2010). Childhood obesity has become a public health crisis (Ogden, 2010), thus, physical activity interventions should make use of the ecological model for physical activity to focus on entire population rather than only the level of the individual.

Limitations. No study has been found that examined the multi-level correlates and interactions across levels of influences among children, and only a few studies have examined the utility of social ecological models for increasing physical activity levels (Glanz et al., 2008; Langille \& Rodgers, 2010). Social ecological models recognize multiple levels of influences on physical activity and multiple levels of intervention opportunities for increasing physical activity. However, these models fail to specify testable relationships among variables (Glanz et al., 2008). This places greater burden for health care professionals to identify critical factors as mechanisms for physical activity interventions. In other words, social ecological model are not practicable, and do not generate testable hypotheses nor provide useful guidelines for application to empirical study.

Summary and conclusion. The theories discussed here each have limitations when applied to physical activity interventions among children. Models of individual health behavior (TPB and SDT) cannot inform the development of physical activity 
intervention strategies that target changes beyond the individual level. The SCT recognizes the influences of environment on behaviors, but it focuses on human's potential abilities to change and construct environment to meet their needs (Glanz et al., 2008). For children who have little power to control their lives and change the environment, behaviors are more passively affected by the environment. Though social ecological models recognize the multiple levels of influences on healthy behaviors, they do not provide testable hypotheses and specific guidelines on which variables are most important for physical activity among children (Elder et al., 2007). To improve the effectiveness of a model to guide behavioral change, the model must be tailored to specific populations and behaviors (Sallis et al., 2006). However, few theories and models used to guide research on physical activity have been developed for children (Welk, 1999). Most studies among children have applied models that were developed for adults, with the assumption that they would be applicable to children (Welk, 1999). It is premature to assume that behaviors of children, adolescents and adults are influenced in the same way.

\section{Precede-Proceed Health Promotion Planning Model}

The Precede-Proceed health promotion planning model is an educational and ecological approach to promote public health and facilitate population health planning, and has served as a successful model in the planning of numerous programs in communities, occupational settings, educational settings, and health care settings (Green \& Kreuter, 2005). This model has four characteristics: (1) flexible and scalable: the model is applicable to all kinds of health programs across a wide range of populations and settings; (2) evidence-based and can be evaluated or tested and then modified based 
on rigorous evaluation: interventions or programs guided by this model are designed based on thorough assessments and can be adjusted based on evaluation; (3) commitment to the principle of participation: this model considers participants' opinions, thoughts and purposes when designing interventions or programs; and (4) a platform for evidencebased "best practice": this model provides a theoretical framework for designing effective interventions regardless of culture, socioeconomic condition, or historical precedent in social customs, laws and policies (Green \& Kreuter, 2005).

The Precede-Proceed model has two components: PRECEDE (for Predisposing, Reinforcing and Enabling Constructs in Educational/ecological Diagnosis and Evaluation), namely, the planned assessments; and PROCEED (for Policy, Regulatory and Organizational Constructs in Educational and Environmental Development), that is, the strategic implementation of multiple actions. The PRECEDE component consists of four phases: Phase 1 social assessment and situational analysis, Phase 2 epidemiological assessment, Phase 3 educational and ecological assessment, and Phase 4 administrative \& policy assessment and intervention alignment, while the PROCEED component includes: Phase 5 implementation, Phase 6 process evaluation, Phase 7 impact evaluation, and Phase 8 outcome evaluation (Green \& Kreuter, 2005). Therefore, the PRECEDE phases can be viewed as the formative evaluation in which diagnostic and assessment data is used to set intervention priorities, objectives, targets and baseline measures for later evaluation. The PROCEED phases can be viewed as summative evaluations in which the principles and methods of management and evaluation are described (Green \& Kreuter, 2005). 
In Phase 1, researchers assess the cultural and social circumstances, concerns and needs of the target population. In Phase 2, researchers need to identify the health goals, problems of the target population and relevant determinants of health. Through these two phases, researchers create the objectives of an intervention. In Phase 3, researchers identify the behaviors and environmental factors that should be changed to promote health, determine which of them is most likely to be amendable to change, and identify the factors influencing the manageable behaviors and environmental factors, and finally plan an intervention to address these factors. In Phase 4, researchers assess the policy, organizational and administrative capabilities and resources that can affect the successful conduct of the intervention. In Phase 5, researchers implement the intervention. From Phase 6 to 8 , researchers evaluate the process of the intervention, the impact of the intervention on targeted behavior and environmental factors, and the health outcomes of the intervention; and adjust the intervention accordingly. In the Precede-Proceed model, evaluation is not viewed as a separate enterprise; rather, it is integrated as a basic component throughout all phases of the model (Green \& Kreuter, 2005).

Phase 3 in this process merits further description. In Phase 3 educational and ecological assessment, three groups of factors are proposed - predisposing factors, reinforcing factors, and enabling factors - that influence health behavior. Predisposing factors include “a person's or population's knowledge, attitudes, beliefs, values and perceptions that facilitate or hinder motivation for change" (Green \& Kreuter, 2005, p. 14), such as early childhood experiences, self-efficacy, and outcome expectancy. Reinforcing factors are the "rewards and feedback the learner receives from others following adoption of a behavior, which may encourage or discourage continuation of the 
behavior" (Green \& Kreuter, 2005, p. 15). Enabling factors are "those skills, resources or barriers that can help or hinder the desired behavioral change as well as environmental change" (Green \& Kreuter, 2005, p. 15), such as facilities and personal or community resources, income or health insurance, laws and statutes, and skills required for a desired behavior.

\section{Youth Physical Activity Promotion Model (YPAP)}

The YPAP model was proposed by Welk to serve as a bridge between theory and practice among children and adolescents (Welk, 1999). The model was developed using the Precede-Proceed health promotion planning model (Green \& Kreuter, 2005) as an organizational framework. It adopts a social-ecological perspective to highlight the influences of personal, social and environmental factors on physical activity. Compared to the SCT, the YPAP model provides a broader perspective on the determinants of physical activity among children and adolescents (Welk, 1999).

The components of the YPAP model and their relationships are displayed in Figure 1. This model proposes that three groups of factors - predisposing factors, reinforcing factors, and enabling factors - are directly correlated with physical activity among children and adolescents (Welk, 1999). Predisposing factors are categorized into two fundamental questions: Is it worth it? and Am I able? The first question addresses the benefits and costs of participating in physical activity and includes a cognitive component, such as attitudes, perceived benefits and beliefs about physical activity, and an affective component, such as physical activity enjoyment and interest. The second question addresses perceived competence such as self-efficacy and physical self-worth. Children high in the two components - cost/benefits and competence - are likely to possess an 
active identity and be predisposed to physically active lifestyles. Reinforcing factors include the influences of families, peers and coaches, and influence physical activity directly, as well as indirectly through predisposing factors. Enabling factors include environmental determinants such as access to equipment, parks and programs, weather, presence of sidewalks and environmental safety; and biological determinants such as physical fitness, skills and body fat. Environmental determinants are necessary but not sufficient determinants of physical activity, but do directly influence physical activity. Enabling factors directly influence physical activity, while biological determinants are more likely to influence physical activity indirectly through perceptions of competence, Am I able? In addition, Welk (1999) proposed including demographic factors in the model because these factors (sex, age, culture and socioeconomics) indirectly influence physical activity levels through predisposing factors, reinforcing factors, and enabling factors. 


\section{Figure 1}

The Youth Physical Activity Promotion Model (Welk, 1999)

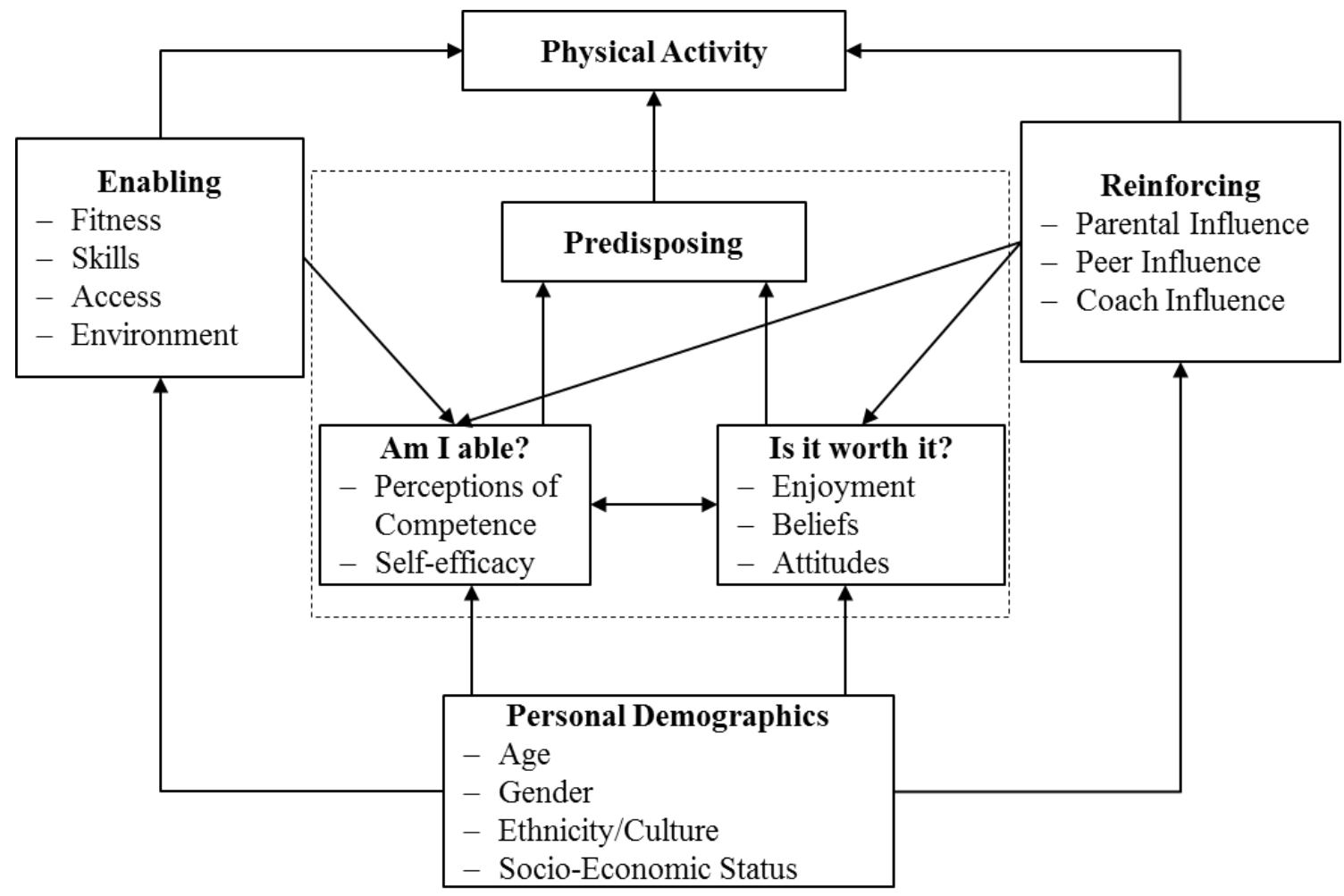

\section{Empirical Uses of YPAP Model}

Three studies investigating the determinants of physical activity among adolescents have used the YPAP model as a theoretical framework. One study among 296 American adolescents was designed to test relationships among attraction to physical activity, perceived physical competence, physical self-worth, parental encouragement, and physical activity (Rowe et al., 2007). Results indicated that attraction to physical activity and perceived physical competence were significantly correlated with physical activity levels, as recorded by pedometers, and parental encouragement was significantly correlated with attraction to physical activity, perceived physical competence and perceived self-worth. Another study in 158 American middle school students was conducted to explore the predisposing, reinforcing and enabling factors related to after- 
school physical activity participation (King, 2008). The results showed that physical activity self-efficacy, attraction to physical activity, and access to sports equipment were the significant determinants of after-school physical activity. The last study was conducted in 601 Thai adolescents to understand the predictors of physical activity (Wattanasit, 2009). The results indicated that attraction to physical activity, perceived physical competence, peer influence, and parental influence accounted for $15 \%$ variance in physical activity. Perceived physical competence affected physical activity indirectly through attraction to physical activity, and peer and parental influence affected physical activity through perceived physical competence and attraction to physical activity. All three of these studies suggest that attraction to physical activity, physical activity selfefficacy, perceived physical competence, access to sports equipment, peer influence, and parental influence are important determinants of physical activity in adolescents.

Only one study among 994 American children has examined the mechanism of parental influence on physical activity (Welk et al., 2003). The study's findings showed that attraction to physical activity, perceived physical competence, and parental influence together explain $30 \%$ of the variance in physical activity among children, explaining a larger proportion of the variance in physical activity among boys than among girls. Attraction to physical activity explains the largest unique portion of the variance in physical activity compared to the other two factors. In addition, parental influence explains $20 \%, 26 \%$, and $28 \%$ of variance in physical activity, attraction to physical activity, and perceived physical competence respectively, indicating that parental influence has an indirect effect on physical activity in children (Welk et al., 2003). 
These three studies among adolescents have focused on predisposing factors (attraction to physical activity, physical activity self-efficacy, and perceived physical competence), reinforcing factors (peer influence and parental influence), and enabling factors (access to sports equipment) to explore this model, and their findings support the assumed relationships among these variables proposed by the model. The only one study was identified among children; it focused on reinforcing factors (parental influence), and predisposing factors (perceived physical competence and attraction to physical activity). Moreover, the purpose of this single study among children was to examine the mechanism of parental influence on physical activity. Therefore, it is necessary to explore the YPAP model among children through examining the relationships among all three sets of factors, predisposing factors, reinforcing factors, and enabling factors, with physical activity.

All these studies use the variable attraction to physical activity to address the question - Is it worth it? In the original YPAP model, Welk used physical activity enjoyment to address this question, although later he used attraction in the parental influence study. The investigator in this study emailed Welk to clarify this question, and he indicated that physical activity enjoyment should influence physical activity equally as does attraction to physical activity (Personal Communication, March 21, 2012). Thus, based on the literature review and comparison of the enjoyment scale with attraction scale, physical activity enjoyment was selected for use in this dissertation study.

\section{Conceptual Framework for the Dissertation Study}

The conceptual framework for the dissertation study was adapted from the YPAP model (See Figure 2). The conceptual framework establishes the relationships among 
physical activity self-efficacy, physical activity enjoyment, parental influence,

environment, personal demographics, and physical activity that will be examined. In this conceptual framework, physical activity self-efficacy, physical activity enjoyment, parental influence, and environment are the significant determinants of physical activity among children. These factors were selected for the study based on the following rationale.

The SCT provides a comprehensive framework for understanding determinants of health-related behaviors (Baranowski et al., 2003). Self-efficacy is the primary concept of the SCT for changing behavior, and it has been shown to be the most significant correlate and predictor of physical activity among children (Rimal, 2001; Strauss et al., 2000). Therefore, self-efficacy was used to answer the first question - Am I able? Physical activity enjoyment is the primary outcome expectancy and intrinsic motivation for children to be active (Kendzierski \& Decarlo, 1991; Motl et al., 2001). Thus, physical activity enjoyment was used to answer the second question - Is it worth it? The two questions - Is it worth it? and Am I able? - were used to address the predisposing factors of physical activity. For younger children whose behaviors are under less volitional control, parents are the primary providers of supportive opportunities for children to be active (Beets, Cardinal, et al., 2010). Specifically, parents serve as "gate keepers" to physical activity through controlling access to physical activity programs, facilities, and outdoor environment; and they serve as models, reinforcers and advocates of healthenhancing behaviors (Beets, Cardinal, et al., 2010; Boufous et al., 2004; Welk et al., 2003). Thus, parental influence was used to address the reinforcing factors of physical activity. Environment, including equipment accessibility and neighborhood safety, plays 
an important role in providing opportunities for children to be active, and facilitates children's use of accessible equipment or supplies, thus improving children's physical activity levels (Committee on Environmental Health, 2009; Molnar et al., 2004). Biological determinants are excluded as enabling factors because physical fitness and body composition are most often considered as outcomes of physical activity. Likewise, skill is excluded from using as an enabling factor due to the difficulty in measuring skills (Baranowski et al., 2003). Thus, environment, including equipment accessibility and neighborhood safety, was selected as the enabling factor in this study.

Finally, personal demographics - child's age, sex, ethnicity, race, number of siblings or children in household, socio-economic status, parents' education levels, marital status and employment status - were collected, because children's physical activity levels have been significantly correlated with children's age, sex, ethnicity, race, sibling's presence, family socio-economic status, parents' marital status, employment status and education levels (Duke \& Huhman, 2003; Hesketh et al., 2006; McVeigh et al., 2004; Troiano et al., 2008). Personal demographics were used to examine how these factors influenced other variables, which might provide valuable information for designing physical activity interventions tailored to children with differing demographic characteristics. Moreover, children's transportation patterns from home to school was assessed, because getting to or from school is the most universal opportunity for children to participate in incidental physical activity (Committee on Environmental Health, 2009). Finally, children's sedentary behavior was assessed to examine its relationship with obesity status. The YPAP conceptual framework was adapted to reflect these choices. See Figure 2 for the adapted conceptual model used to guide the dissertation study. Table 1 
compares the conceptual and operational definitions between the YPAP model and the conceptual framework used in the dissertation study.

\section{Figure 2}

The Conceptual Framework for the Study, Adapted from the YPAP Model (Welk, 1999)

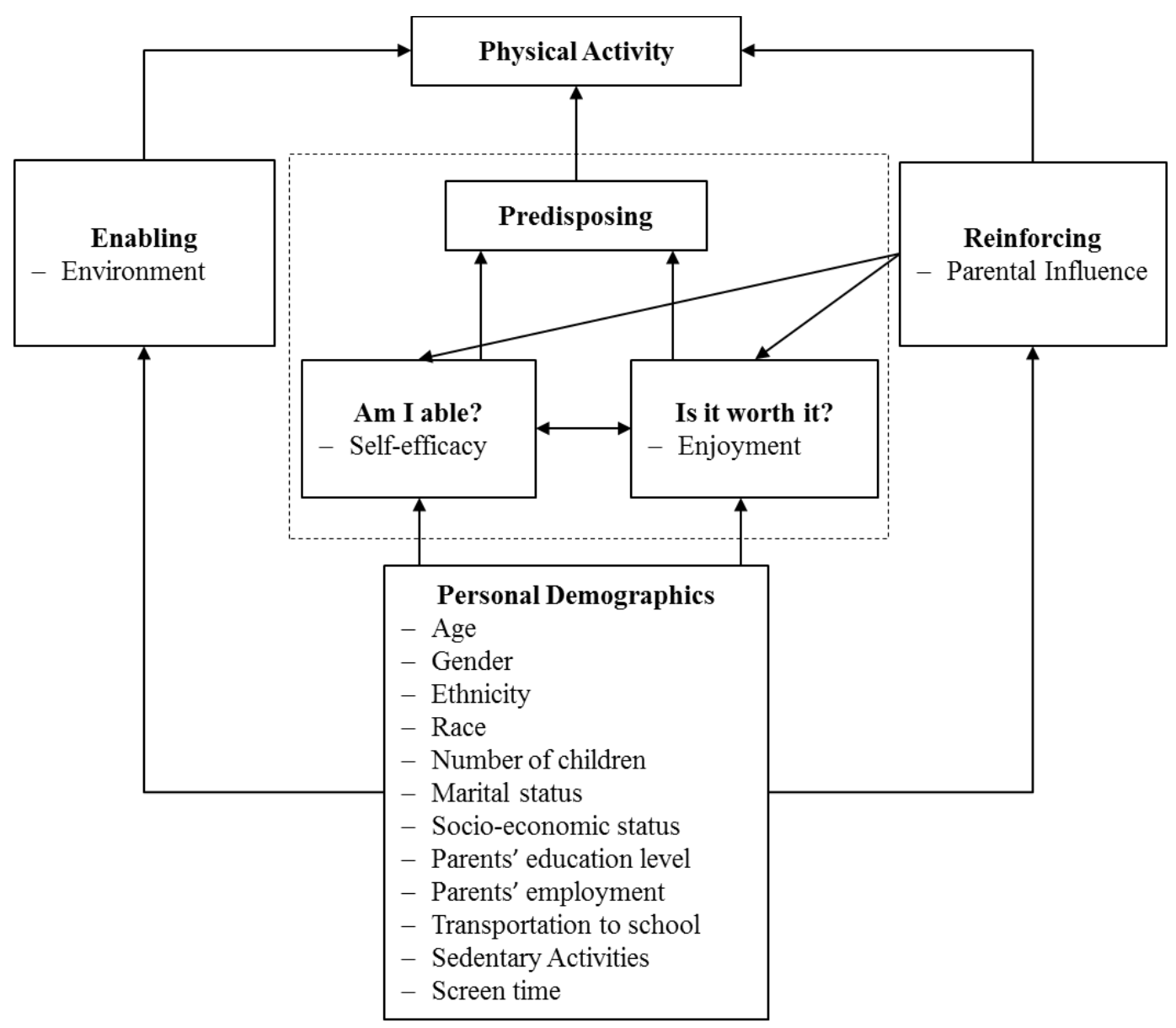


Table 1

Comparisons of Conceptual and Operational Definitions between the YPAP Model and the Dissertation Conceptual Framework

\begin{tabular}{|c|c|c|c|}
\hline Concept & Definition & YPAP Model & Conceptual Model for the Dissertation Study \\
\hline \multirow[t]{2}{*}{$\begin{array}{l}\text { Physical } \\
\text { Activity }\end{array}$} & Conceptual & $\begin{array}{l}\text { Physical activity is any bodily movement } \\
\text { produced by skeletal muscles that results in } \\
\text { energy expenditure (Caspersen et al., 1985, p. } \\
\text { 126). }\end{array}$ & $\begin{array}{l}\text { Physical activity is any bodily movement } \\
\text { produced by skeletal muscles that results in } \\
\text { energy expenditure (Caspersen et al., 1985, p. } \\
\text { 126). }\end{array}$ \\
\hline & Operational & $\begin{array}{l}\text { Physical Activity Questionnaire for Older } \\
\text { Children (PAQ-C) (Crocker, Bailey, Faulkner, } \\
\text { Kowalski, \& McGrath, 1997; Kowalski, Crocker, } \\
\text { \& Faulkner, 1997) }\end{array}$ & $\begin{array}{l}\text { Physical Activity Questionnaire for Older } \\
\text { Children (PAQ-C) (Crocker et al., 1997; } \\
\text { Kowalski et al., 1997) }\end{array}$ \\
\hline \multirow[t]{2}{*}{ Self-Efficacy } & Conceptual & $\begin{array}{l}\text { Perceived self-efficacy is belief in one's own } \\
\text { capacity to organize and execute a course of } \\
\text { action required to achieve a goal (Bandura, 1997, } \\
\text { p. 3). Self-efficacy includes self-regulatory } \\
\text { efficacy and barrier self-efficacy (DuCharme \& } \\
\text { Brawley, 1995). }\end{array}$ & $\begin{array}{l}\text { Self-efficacy is confidence in one's own ability to } \\
\text { be physically active. It has three components: } \\
\text { support seeking, barriers and positive alternatives } \\
\text { (Saunders et al., 1997). }\end{array}$ \\
\hline & Operational & $\begin{array}{l}\text { Physical Activity Self-efficacy Scale (PASES) } \\
\text { (Saunders et al., 1997) }\end{array}$ & $\begin{array}{l}\text { Physical Activity Self-Efficacy Scale (PASES) } \\
\text { (Saunders et al., 1997) }\end{array}$ \\
\hline \multirow[t]{2}{*}{$\begin{array}{l}\text { Physical } \\
\text { Activity } \\
\text { Enjoyment }\end{array}$} & Conceptual & $\begin{array}{l}\text { Physical activity enjoyment refers to a positive } \\
\text { affective state that reflects feelings such as } \\
\text { pleasure, liking and fun related to physical } \\
\text { activity (Motl et al., 2001, p. 110). }\end{array}$ & $\begin{array}{l}\text { Physical activity enjoyment refers to a positive } \\
\text { affective state that reflects feelings such as } \\
\text { pleasure, liking and fun related to physical } \\
\text { activity (Motl et al., 2001, p. 110). }\end{array}$ \\
\hline & Operational & $\begin{array}{l}\text { Physical Activity Enjoyment Scale (PACES) } \\
\text { (Kendzierski \& Decarlo, 1991; Moore et al., } \\
\text { 2009) }\end{array}$ & $\begin{array}{l}\text { Physical Activity Enjoyment Scale (PACES) } \\
\text { (Kendzierski \& Decarlo, 1991; Moore et al., } \\
\text { 2009) }\end{array}$ \\
\hline
\end{tabular}




\begin{tabular}{llll}
\hline $\begin{array}{l}\text { Parental } \\
\text { Influence }\end{array}$ & Conceptual & $\begin{array}{l}\text { Role modeling, encouragement, facilitation (take } \\
\text { children to parks), and involvement (exercise } \\
\text { with children) (Welk, 1999). } \\
\text { Parental Influence Scale (Welk et al., 2003) }\end{array}$ & $\begin{array}{l}\text { Role modeling, encouragement, facilitation and } \\
\text { involvement (Welk et al., 2003). }\end{array}$ \\
Environment & Operational & $\begin{array}{l}\text { Access to equipment, parks and physical activity } \\
\text { programs; availability of play spaces; good } \\
\text { weather; presence of sidewalks; safety (Welk, }\end{array}$ & $\begin{array}{l}\text { Equipment accessibility (family, school and } \\
\text { community), and neighborhood safety }\end{array}$ \\
$\begin{array}{l}\text { Personal } \\
\text { Demographics }\end{array}$ & Operational & $\begin{array}{l}\text { Age, sex, ethnicity/culture, and socio-economic } \\
\text { status (Welk, 1999) }\end{array}$ & $\begin{array}{l}\text { Age, sex, ethnicity, race, number of children, } \\
\text { marital status, socio-economic status, parents } \\
\text { education level and employment status }\end{array}$ \\
\hline
\end{tabular}




\section{Summary}

Regular physical activity has many desirable influences on children's growth, health, and academic performance. However, the majority of American children do not meet the daily physical activity recommendation, and only three states require the nationally recommended levels of physical education. Overweight or obese children engage in lower levels of physical activity compared to non-overweight children, indicating that lack of physical activity may be one significant contributor to the childhood obesity epidemic. Therefore, increasing physical activity is an important factor in efforts to control childhood obesity.

Considering the trend toward increased prevalence of childhood obesity and the promising role of physical activity in controlling childhood obesity, many physical activity interventions have been designed and conducted to check the childhood obesity epidemic. However, the majority of physical activity interventions remain largely ineffective in controlling childhood obesity. To design effective physical activity interventions among children, it is important for interventions to be based on a theoretical model that adequately explains the variance of physical activity among a specific population. Thus, a theoretical framework of the determinants of physical activity in children is needed to guide further intervention design.

Commonly used theories have limitations when applying to physical activity interventions among children. Models of individual health behavior (TPB and SDT) cannot inform the development of physical activity intervention strategies that target changes beyond the individual level. The SCT recognizes the influence of environment on behaviors, but it focuses on human's potential abilities to change and construct 
environment to meet their needs. For children who have little power to control their lives and change the environment, behaviors are more passively affected by the environment. Though social ecological models recognize the multiple levels of influence on healthy behaviors, they do not provide testable hypotheses and specific guidelines on which variables may be targeted to improve physical activity among children. To improve the effectiveness of a model in guiding behavioral change, the model must be tailored to specific populations and behaviors. However, few theories and models used to guide research on physical activity are specifically developed for children.

The YPAP model was developed specifically for promoting physical activity among children and adolescents by considering their developmental, psychological and behavioral characteristics. However, only one study has been found using this model in children, and that study focuses only on reinforcing factors (parental influence), and predisposing factors (perceived physical competence and attraction to physical activity) to examine this model, but not enabling factors. Considering the important role of environment in providing opportunities for children to be physically active and to facilitate children's use of accessible equipment or supplies, it is necessary to explore this model among children by examining the relationships among predisposing factors, reinforcing factors, enabling factors and physical activity.

The potential determinants of physical activity to be investigated in this study are based on the YPAP model and grounded in previous empirical research. Physical activity self-efficacy is the strongest predictor of physical activity among children, and physical activity enjoyment is the primary outcome expectancy and intrinsic motivation for children to be active. Thus, physical activity self-efficacy and physical activity enjoyment 
are potential predisposing factors. For younger children whose behaviors are under less volitional control, parents are the primary providers of supportive opportunities for children to be active. Thus, parental influence is a possible reinforcing factor. The environment plays an important role in providing opportunities for children to be active and is a likely enabling factor. 


\section{CHAPTER III}

\section{METHODOLOGY}

The purpose of this study was to explore the psychosocial and environmental determinants of physical activity among children aged 8 to 11 years old. Relationships among predisposing factors (physical activity self-efficacy and physical activity enjoyment), reinforcing factor (parental influence), enabling factor (environment), and physical activity in elementary school children were examined to provide insights for developing future interventions to increase physical activity and reduce obesity in this vulnerable population. This chapter presents the research design, setting, sample, measurements, procedures, data analysis, and protection of human subjects for this dissertation study.

\section{Research Design}

A cross-sectional correlational design (path analytic study) was used to explore the relationships among determinants of physical activity in children aged 8 to 11 years old. This method was selected because it is a means to test a "hypothesized causal chain among a set of independent variables, mediating variables and a dependent variable" (Polit \& Beck, 2004, p. 191). The dependent/outcome variable was physical activity, and the major independent variables were physical activity self-efficacy, physical activity enjoyment, parental influence and environment, with physical activity self-efficacy and physical activity enjoyment partially mediating the effect of parental influence on 
physical activity. In addition, a pretest-posttest design was used to examine the influence of wearing pedometers on children's perceptions of physical activity self-efficacy, physical activity enjoyment, parental influence and environment in a randomly selected subsample from all the participating children.

In this study, all the children were asked for responses to a series of standardized instruments, named as the posttest surveys, which were administered after participants wore pedometers for seven consecutive days. The posttest surveys measured physical activity self-efficacy, physical activity enjoyment, parental influence, environment and self-reported physical activity. The parents or the legal guardians were asked for responses to a demographic information questionnaire. The randomly selected subsample of 50 children were also asked to complete a series of standardized instruments, named as the pretest surveys, which measured physical activity self-efficacy, physical activity enjoyment, parental influence and environment before receiving a pedometer.

\section{Setting}

The Communities In Schools of Clark County (CISCC) is a non-profit organization using community resources to help children learn successfully, stay in school, and prepare them for future life (Communities In Schools of Clark Co., 2010). Since 2001, the CISCC has provided afterschool programs or extended day learning services in one primary school (Pre-Kindergarten $-1^{\text {st }}$ grade), sixteen elementary schools (Pre-Kindergarten $-5^{\text {th }}$ grade), three middle schools $\left(6^{\text {th }}-8^{\text {th }}\right.$ grade), and one high school $\left(9^{\text {th }}-12^{\text {th }}\right.$ grade) in Greater Clark County, Indiana (Communities In Schools of Clark Co., 2010). In 2011, Greater Clark County included 42,909 households with an average annual household income of $\$ 60,340$, compared to $\$ 61,977$ in the state as a whole and 
$\$ 72,579$ nationally. About $11.9 \%$ of people in the Greater Clark County were below poverty level compared to $14.1 \%$ in Indiana and $14.3 \%$ nationally (U.S. Census Bureau, 2013).

Nine suburban elementary schools and one rural elementary school with afterschool programs were selected to obtain a sample representative of the county in this study (Phan \& Glander, 2007). Rural district was defined as a territory that is less than or equal to 25 miles from an urbanized area of 50,000 or more people, as well as territory that is less than or equal to 10 miles from an urban cluster of at least 2,500 and less than 50,000 people (Phan \& Glander, 2007). Suburban district was defined as a territory outside a principal city (primary population and economic center of an Metropolitan Statistical Area) and inside an urbanized area (with a population of 50,000 or more) (Phan \& Glander, 2007). The ten elementary schools were located in two townships, Clarksville and Jeffersonville, within Greater Clark county (National Center for Educational Statistics [NCES], 2012). The demographics of these two townships are displayed in Table 2 (The Office of Fair Housing and Equal Opportunity, 2012).

In the 2011-2012 school year, in the ten elementary schools selected for this study, there were a total of 4,287 children (2,251 boys and 2,037 girls) with 697 children in Grade 3, 736 in Grade 4, and 610 in Grade 5 (NCES, 2012). Among the ten elementary schools, $65.7 \%(n=2,816)$ of the children were Caucasians, $15.4 \%(n=659)$ were African Americans, and 8.2\% $(n=351)$ were Hispanics. In comparison, nationally, 53\% of 8-11 year-old children are Caucasians, 14\% are African Americans, and 24\% are Hispanics (Kaiser Family Foundation, 2011b). Almost 52\% $(n=2,224)$ of the children were eligible for free lunch compared to $39.0 \%$ in Indiana, and $9.9 \%(n=426)$ were 
eligible for reduced-price lunch compared to $7.7 \%$ in Indiana (NCES, 2012; The Annie E.

Casey Foundation, 2013).

\section{Table 2}

Demographic Summary of Communities

\begin{tabular}{|c|c|c|}
\hline Variable & Clarksville & Jeffersonville \\
\hline Population & 19,177 & 45,173 \\
\hline 6-11 years old & $7.4 \%$ & $7.7 \%$ \\
\hline Sex (male) & $48.3 \%$ & $48.9 \%$ \\
\hline Ethnicity (Hispanic) & $5.0 \%$ & $3.0 \%$ \\
\hline $\begin{array}{l}\text { Race } \\
\text { Caucasian } \\
\text { African American }\end{array}$ & $\begin{array}{c}85.2 \% \\
7.4 \%\end{array}$ & $\begin{array}{l}79.9 \% \\
13.4 \%\end{array}$ \\
\hline $\begin{array}{l}\text { Marital Status } \\
\text { Married (male) } \\
\text { Married (female) } \\
\text { Divorced/Widowed (male) } \\
\text { Divorced/Widowed (female) } \\
\text { Single (male) } \\
\text { Single (female) }\end{array}$ & $\begin{array}{c}24.5 \% \\
24.5 \% \\
8.3 \% \\
16.4 \% \\
14.8 \% \\
11.5 \%\end{array}$ & $\begin{array}{c}27.1 \% \\
27.4 \% \\
7.4 \% \\
12.6 \% \\
13.7 \% \\
11.9 \%\end{array}$ \\
\hline $\begin{array}{l}\text { Education } \\
\text { Less than high school graduate } \\
\text { High school graduate } \\
\text { Some college } \\
\text { Associate degree } \\
\text { Bachelor's degree } \\
\text { Graduate/professional degree }\end{array}$ & $\begin{array}{c}18.9 \% \\
37.4 \% \\
22.9 \% \\
7.4 \% \\
9.0 \% \\
4.4 \%\end{array}$ & $\begin{array}{c}13.5 \% \\
36.0 \% \\
25.5 \% \\
7.8 \% \\
11.8 \% \\
7.4 \%\end{array}$ \\
\hline $\begin{array}{l}\text { Employment } \\
\text { Unemployed (male) } \\
\text { Unemployed (female) }\end{array}$ & $\begin{array}{l}3.1 \% \\
2.5 \%\end{array}$ & $\begin{array}{l}3.4 \% \\
2.5 \%\end{array}$ \\
\hline $\begin{array}{l}\text { Household Income } \\
\text { Average } \\
<\$ 25,000 \\
<\$ 50,000\end{array}$ & $\begin{array}{l}\$ 55,920 \\
27.3 \% \\
58.9 \%\end{array}$ & $\begin{array}{l}\$ 61,737 \\
21.4 \% \\
48.7 \%\end{array}$ \\
\hline Population Density & 1,963 & 1,238 \\
\hline Total Crime Risk Index* & 130 & 61 \\
\hline
\end{tabular}


Note. * Total Crime Risk Index is used to assess an area's relative safety related to national average of 100. It is an index of the combined risks of rape, murder, assault, robbery, burglary, larceny, and vehicle theft.

\section{Sample Size}

The hypothesized path model for this study is shown in Figure 3 below. The requirement of sufficient sample size for Structural Equation Modeling (SEM), which is

"a statistical methodology that takes confirmatory (i.e., hypothesis-testing) approach to analysis of a structured theory bearing on some phenomenon" (Byrne, 2010, p. 3), is to have 10 to 1 ratio for the number of subjects to the number of parameters (= number of paths (the straight arrow) + number of variances of exogenous + number of covariance + number of disturbance terms) (Suhr, 2012). In this study, there are 11 parameters (six paths + two exogenous variables + zero covariance + three disturbance terms), so the sample size of 110 was determined to be sufficient to utilize the statistical technique of SEM. For the feasibility study (Ling, 2012), 16.7\% of questionnaires had missing data, thus, 23 additional participants were recruited to obtain adequate power. Therefore, a total of 133 children were recruited in this study. The power analysis for the pairedsamples t-test with $a=.05$ and moderate effects size (.40-.50) indicated that a sample size of 50 was adequate to gain a power of .80 or above (See Figure 4). Therefore, 50 children were randomly selected from the 133 children to participate in the pretest-posttest study. 


\section{Figure 3}

\section{The Hypothesized Path Model}

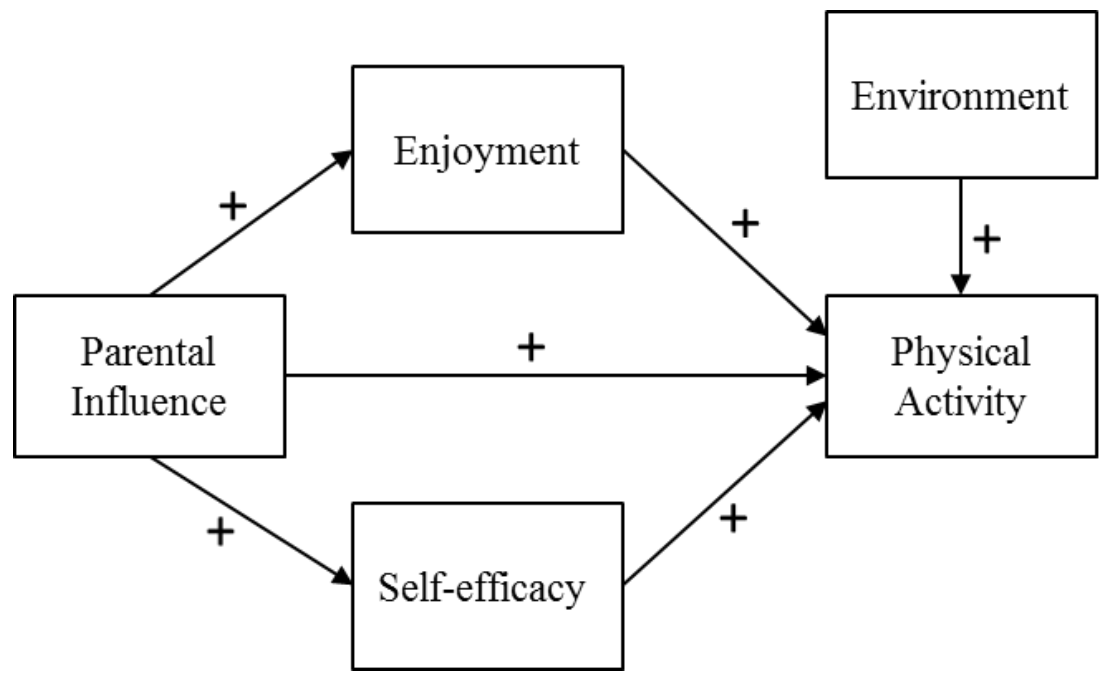

Figure 4

\section{$\underline{\text { Power Analysis for Paired-samples } t \text {-test }}$}

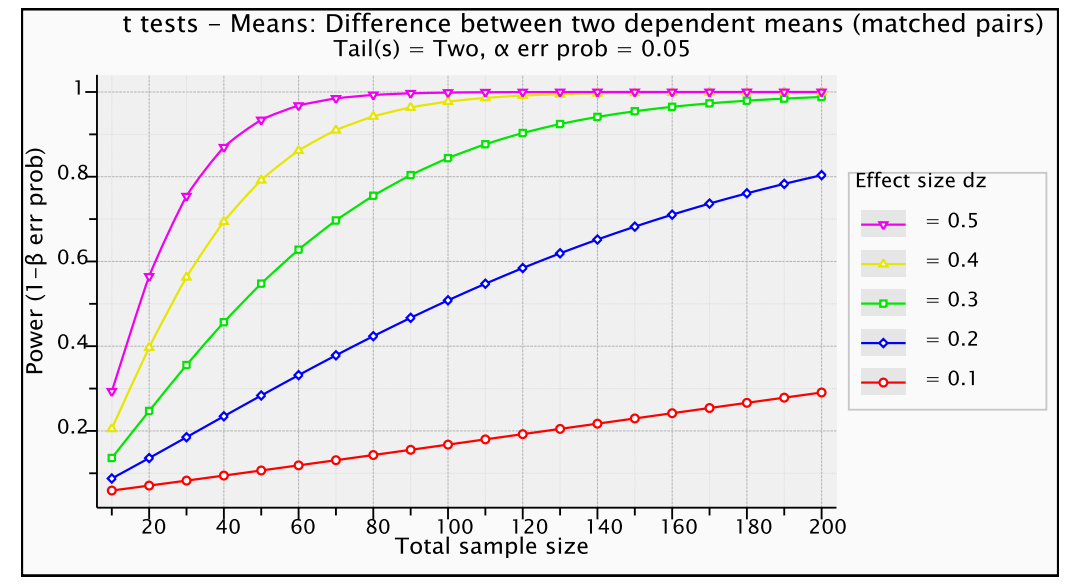

\section{Sample}

Participants in this study were children aged 8 to 11 years old. This age group was chosen because: (a) obesity rates are higher among children aged 6 to 11 years old than among other age groups (Ogden, 2010); (b) physical activity levels in American children 
drop sharply between age 9 and age 15 (Bock \& Miller, 2008); and (c) after age 8, children can read with considerable ease and comprehend complex and compound sentences (Rodgers, 2007). Thus, considering the prevalence of childhood obesity, levels of physical activity and language development, children aged 8 to 11 years old were chosen for this study. To gain a representative sample, cluster sampling (a process of selecting specific sites/clusters and recruiting every individual at the selected sites/clusters into the study) (Polit \& Beck, 2004) was used to select 133 participants from ten elementary schools with CISCC afterschool programs. The sample size of $N=$ 133 was estimated using power analysis, based on recommended sample size for the planned statistical method, SEM - path analysis. Inclusion criteria were: (1) 8 to 11 years old; (2) can speak and read English; (3) absence of health problems that prevent participation in physical activity; (4) willingness to assent to participate in the study; and (5) ability to obtain signed parental consent. Input from CISCC program director and teachers was used to help determine children's eligibility to participate in the study based on the inclusion criteria. Simple random sampling (a table of random numbers) was used to select 50 children from the participating sample for the pretest-posttest study. The sample size of 50 was estimated by the power analysis using the paired-samples t-test.

A total of 133 children, 59 girls and 74 boys, participated in this study with an average age of $9.25(S D=.93)$ years old. Six children, one in the pretest-posttest study, could not complete the posttest surveys due to a health problem, injury or family relocation to a different geographical area. About $20.3 \%$ of the children were Hispanics, and 25.6\% were African Americans. More than half of the families were married, with an average of three children. Approximately $42.8 \%$ of the families had annual family 
income less than $\$ 30,000$, and $64.6 \%$ had annual family income less than $\$ 50,000$.

Twenty-four fathers (18\%) and $20(15 \%)$ mothers were unemployed. Approximately 50.3\% of the fathers and $42.9 \%$ of the mothers received a high school diploma or less, while $21.1 \%$ of the fathers and $21.8 \%$ of the mothers received some college education. Table 3 describes the demographic characteristics of all the participants $(N=133)$ and children participating in the pretest-posttest study $(n=50)$. No significant demographic differences were found between the two groups of children, indicating that the pretest sample was representative to the total sample in this study. 
Table 3

Demographic Characteristics of Participants

\begin{tabular}{|c|c|c|c|}
\hline Variable & $\begin{array}{l}\text { Participants }(N=133) \\
\quad N(\%) \text { or } M(S D)\end{array}$ & $\begin{array}{c}\text { Subsample Participants }(n=50) \\
N(\%) \text { or } M(S D)\end{array}$ & $p$-value \\
\hline Age & $9.26(.93)$ & $9.28(.90)$ & .874 \\
\hline Sex (Female) & $59(44.4 \%)$ & $21(42.0 \%)$ & .775 \\
\hline Ethnicity (Hispanic) & $27(20.3 \%)$ & $11(22.0 \%)$ & .801 \\
\hline $\begin{array}{ll}\text { Race } & \\
\text { White or Caucasian } \\
\text { Black or African } \\
\text { Asian/Pacific Island } \\
\text { Mixed-race } \\
\text { Other }\end{array}$ & $\begin{array}{l}68(51.1 \%) \\
34(25.6 \%) \\
2(1.5 \%) \\
11(8.3 \%) \\
10(7.5 \%)\end{array}$ & $\begin{array}{c}26(52.0 \%) \\
8(16.0 \%) \\
0 \\
7(14.0 \%) \\
4(8.0 \%)\end{array}$ & .925 \\
\hline Number of Children & $2.71(1.42)$ & $2.32(1.00)$ & .080 \\
\hline $\begin{array}{l}\text { Parents' Marital Status } \\
\text { Married/partnered } \\
\text { Separated/divorced/widowed } \\
\text { Single }\end{array}$ & $\begin{array}{l}76(57.1 \%) \\
27(20.3 \%) \\
30(22.6 \%)\end{array}$ & $\begin{array}{c}29(58.0 \%) \\
7(14.0 \%) \\
14(28.0 \%)\end{array}$ & .845 \\
\hline $\begin{array}{l}\text { Annual Family Income } \\
\quad \leq \$ 19,999 \\
\$ 20,000-\$ 29,999 \\
\$ 30,000-\$ 49,999 \\
\geq \$ 50,000\end{array}$ & $\begin{array}{l}37(27.8 \%) \\
20(15.0 \%) \\
29(21.8 \%) \\
41(30.8 \%)\end{array}$ & $\begin{array}{c}13(26.0 \%) \\
9(18.0 \%) \\
11(22.0 \%) \\
15(30.0 \%)\end{array}$ & .999 \\
\hline
\end{tabular}


Father Employment Status

Full Time

$78(58.6 \%)$

.315

Part Time

$17(12.8 \%)$

$31(62.0 \%)$

$5(10.0 \%)$

No

$24(18.0 \%)$

$6(12.0 \%)$

Mother Employment Status

Full Time

Part Time

$87(65.4 \%)$

$35(70.0 \%)$

$26(19.5 \%)$

$11(22.0 \%)$

No

$20(15.0 \%)$

$4(8.0 \%)$

\section{Father Education Level}

Less than high school graduate

$20(15.0 \%)$

$5(10.0 \%)$

$47(35.3 \%)$

$28(21.1 \%)$

Some college

Technical school or community college

$9(6.8 \%)$

$11(8.3 \%)$

$4(3.0 \%)$

.429

Bachelor's degree

Graduate or professional degree

\section{Mother Education Level}

Less than high school graduate

High school graduate

$21(15.8 \%)$

$20(40.0 \%)$

$12(24.0 \%)$

$1(2.0 \%)$

$3(6.0 \%)$

$1(2.0 \%)$

Some college

Technical school or community college

$36(27.1 \% 0$

$5(10.0 \%)$

$29(21.8 \% 0$

$14(28.0 \%)$

$17(12.8 \%)$

$12(24.0 \%)$

$7(14.0 \%)$

$24(18.0 \%)$

$9(18.0 \%)$

Graduate or professional degree

$6(4.5 \%)$ 


\section{Variables and their Measurement}

It is important to consider reading level when choosing questionnaires for use in studies with children. Thus, only questionnaires developed for children were used in this study. An investigator-developed demographic information questionnaire was used to assess children's demographic characteristics. This demographic questionnaire was completed by parents/guardians, because children might not be able to answer some questions in the questionnaire. BMI calculated using height and weight [(weight $\mathrm{kg}) /(\text { height } \mathrm{m})^{2}$ ] obtained by the investigator was used to assess children's obesity status.

To improve the accuracy of assessment of the outcome variable, physical activity, two methods (self-report and objective) were used to assess children's physical activity levels. Self-report measurements are mostly used to assess physical activity due to low cost, low burden, less time consumption, and ease of administration among large numbers of children (Sallis, 1991). The reason for using pedometers to estimate objective physical activity levels is that pedometers are inexpensive but valid electronic devices for assessing physical activity levels among children (McNamara, Hudson, \& Taylor, 2010). Thus, both a self-report measure (Physical Activity Questionnaire for Older Children) (Crocker et al., 1997) and an objective measure (step counts obtained by pedometers) were used to more accurately assess physical activity levels among children and to evaluate the convergent validity of self-report measurement in assessing children's physical activity levels.

The Physical Activity Self-Efficacy Scale (PASES) developed by Saunders and colleagues (1997) was used to assess physical activity self-efficacy. The Physical Activity Enjoyment Scale (PACES) developed by Moore and colleagues (2009) was used 
to assess physical activity enjoyment in this study. These two scales together were used to assess predisposing factors of physical activity. The Parental Influence Scale (PAIS) developed by Welk and colleagues (2003) was used to assess parental influence, the reinforcing factor. A modified environment scale was used to assess environmental determinants of physical activity, the enabling factor.

When evaluating the internal consistency of questionnaires, the common recommendation from Nunnally and Bernstein (1994) that Cronbach's alpha coefficient of .70 indicates acceptable reliability was used. However, for scales with more than 10 items, the cutoff value of .80 was used, while the cutoff value of .50 was used for subscales with 6 items or fewer, because alpha is positively affected by the number of items (Cortina, 1993). Item-total correlation coefficient (Pearson $r$ ), was used to assess reliability of the items on each scale, with a cutoff value of .30 indicating acceptable reliability or stability (Nunnally \& Bernstein, 1994).

\section{Personal Demographics}

An investigator-developed demographic information questionnaire (See Appendix B), adapted from a prior version used in the feasibility study (Ling, 2012) which was found to have good face validity, was used to collect demographic information. Variables in the questionnaire include: (1) child's age; (2) child's sex; (3) child's ethnicity; (4) child's race; (5) number of children in the family; (6) parent's marital status; (7) annual family income; (8) father's employment status; (9) mother's employment status; (10) father's education level; (11) mother's education level, (12) mode of transportation from home to school; (13) sedentary activities; and (14) screen-time minutes (TV/DVDs, video games and computers). This instrument was completed by children's parents or legal 
guardians, because children might not be able to accurately answer some questions on the questionnaire.

\section{Obesity (BMI)}

BMI was calculated using height and weight [(weight $\left.\mathrm{kg}) /(\text { height } \mathrm{m})^{2}\right]$. The U.S. Preventive Services Task Force indicates that BMI is an acceptable and cost-effective measure to screen for obesity among children aged 6 years and older (U.S. Preventive Services Force, 2010). Thus, the age- and sex-specific percentile for BMI was used to assess children's obesity status (See Appendix C and D) (CDC, 2011a).

\section{Outcome Variable}

Physical activity. Pedometers have been shown to be a reliable and valid method to assess physical activity levels among children (McNamara et al., 2010). No consistent evidence of reactivity is identified in studies using pedometers to assess children's physical activity levels (Craig, Tudor-Locke, Cragg, \& Cameron, 2010; Rowe, Mahar, Raedeke, \& Lore, 2004). In a study with children wearing pedometers for seven consecutive days, $58 \%$ children returned the pedometers and data, $1 \%$ returned data but not pedometers, $12 \%$ returned pedometers but not data, and 30\% returned nothing (Craig et al., 2010). Another study in 1,370 children reported a $44 \%$ compliance rate (Eisenmann, Laurson, Wickel, Gentile, \& Walsh, 2007). One study in 11,669 children aged 5 to 19 children suggests that two days should be sufficient for determining average daily steps for children (Craig et al., 2010). However, some researchers suggest using seven consecutive days or at least one week day and one weekend day (Clemes \& Biddle, 2013). No systematic bias is found for the day of the week selected to start recording 
pedometer steps (Craig et al., 2010). Evidence suggests that step counts less than 1,000 or greater than 30,000 should be considered outliers (Rowe et al., 2004).

The Yamax SW-200 (Yamax Corp., Tokyo, Japan) is the most commonly used pedometer to assess physical activity among children, and has been shown to have moderate to high validity with Pearson correlation coefficients ranging from .39 to .99 with heart rate and oxygen consumption (McNamara et al., 2010; Sirard \& Pate, 2001). All children voluntarily participating in the study were asked to wear a pedometer for seven consecutive days, and the average number of steps was used to describe their levels of physical activity. A pedometer log (See Appendix E) was created for children to record their steps each day. In the pedometer log, children were asked to write down the number of steps indicated on the pedometer each day, and the time and date the number was recorded with the help of investigator or parents/guardians. The pedometer log included detailed instructions for parents/guardians to supervise or help their children to correctly wear the pedometer, record the steps and then reset the pedometer to zero. Evidence suggests that 13,000 steps/day for boys and 11,000 steps/day for girls provide a reasonable estimation of attainment of 60 minutes of moderate- or vigorous-intensity physical activity (Tudor-Locke et al., 2011), thus these cut points were used to assess the percentage of children meeting daily physical activity recommendations in this sample.

A variety of self-report instruments have been developed to assess self-reported physical activity levels among children. Considering the psychometric properties of the PAQ-C in a number of studies among children of similar age, the PAQ-C (See Appendix F) was selected for use in this study. The PAQ-C is a self-administered 7-day recall measurement intended to assess general moderate- to vigorous-intensity physical activity 
among children aged 8 to 14 years old. It uses a five-point Likert scale with higher scores indicating higher levels of physical activity (Crocker et al., 1997). This scale has been shown to have high reliability and moderate validity when used with children aged 8 to 14 years old.

In 1997, Crocker and colleagues designed three studies to test the reliability of this scale. The first study conducted in 215 children aged 9 to $15 y$ years old indicated that the scale had strong internal consistency with Cronbach's alpha coefficient of .82. The second study conducted in 84 children aged 9 to 14 years old indicated that the scale had acceptable test-retest reliability with correlation coefficient of .79. The third study in 200 children aged 8 to 16 years old used the Generalizability theory to investigate reliability, using the average of the three physical activity scores, which were collected in January, April and October-November. Generalizability theory is an extension of the intraclass reliability model. Similar to the Pearson Product moment correlation, the Generalizability coefficient allows unlimited or repeated measures of an instrument to be used to assess reliability. The Generalizability theory provides a framework to examine the extent to which one can assume equivalence of a measurement process across one or more dimensions (DeVellis, 2003). The Generalizability coefficient was .88 indicating that it had acceptable reliability for assessing yearly physical activity in children (Crocker et al., 1997).

Consistent evidence has demonstrated that the PAQ-C has not only strong reliability, but also acceptable validity. Kowalski and colleagues (1997) conducted two additional studies to examine the criterion related validity of this scale in children aged 813 years old. The first study in 89 children aged 8 to 13 years old indicated that the PAQ- 
$\mathrm{C}$ was moderately related to the activity rating $(r=.63)$, the week summation of 24-hour moderate to vigorous activity recall $(r=.53)$, and the teacher's rating of physical activity $(r=.45)$. The second study in 97 children indicated that the PAQ-C was significantly correlated with the activity rating $(r=.57)$, the Leisure Time Exercise Questionnaire ( $r$ $=.41)$, the Canadian home fitness test $(r=.39)$, the seven-day recall interview $(r=.46)$, and the step test of fitness $(r=.28)$ (Kowalski et al., 1997). Therefore, the PAQ-C has strong reliability and acceptable validity.

To date, the PAQ-C has been broadly used in cross-sectional studies and longitudinal studies to examine levels of physical activity among American children aged 6 to 14 years old (Ball, Marshall, \& McCargar, 2003; MacKelvie, Petit, Khan, Beck, \& McKay, 2004). Evidence has supported the conclusion that this scale is a cost efficient method for assessing general levels of physical activity among children aged 6 to 14 years old with strong psychometric properties. In the feasibility study among 24 children aged 8 to 13 years old, the Cronbach's alpha coefficient was .86 (Ling, 2012). Thus, the PAQ-C was selected as the most appropriate tool to measure self-reported physical activity levels in this study.

\section{Predisposing Factors}

Physical activity self-efficacy. The Physical Activity Self-Efficacy Scale (PASES) (See Appendix G) is a self-administered 17-item measure used to assess children's confidence in overcoming barriers to physical activity. It is a dichotomous scale (yes - code 1, or no - code 0 ) with higher score indicating higher physical activity self-efficacy (Saunders et al., 1997). The SCT provides the theoretical foundations for the

development of the PASES. The original PASES (five-point Likert) went through 4 pilot 
tests in fifth grade students, and three modifications - selecting age-appropriate words, simplifying rating scales (from 5-point to 2-point), and putting instruments in a visually more appealing format (larger print, wider margins) - were made to obtain the final version of the instrument. When administering this questionnaire, the investigator reads the instructions and questions to a group of children using a standardized script. The script includes the definition for physical activity "any active games, active play, sports, or exercise that gets you moving, breathing faster, and your heart beating faster", and poster pictures displaying the range of possible physical activities (Saunders et al., 1997, p. 242).

Principal component factor analysis with Varimax rotation suggested a threefactor structure for the scale - support seeking, barriers, and positive alternatives, with all items loading above .35 (Saunders et al., 1997). Moreover, the Cronbach's alpha coefficients were $.71, .71$ and .54 for support seeking, barriers, and positive alternatives respectively. Similarly, the test-retest reliability coefficients were $.76, .82$, and .61 respectively, which indicate that this scale has adequate reliability. Scores on this scale have been reported to be significantly correlated with intention to engage in physical activity, and the barriers subscale was significantly correlated with afterschool physical activity levels (Saunders et al., 1997). The results from both factor analysis and correlations support the conclusion that this scale has adequate construct validity. A national study in 2,257 children aged 9-13 years old reported that the scale had Cronbach's alpha coefficient of .68, and test-retest stability coefficient of .75 (Huhman et al., 2007). Additionally, the scale has been supported to be a reliable and valid scale broadly used in children aged 8 to 13 years old (Huhman et al., 2007; Thompson et al., 
2008). In the feasibility study among 24 children aged 8 to 13 years old, the Cronbach's alpha coefficient was .79 (Ling, 2012). Thus, the PASES has strong psychometric properties and can be used to assess physical activity self-efficacy in children aged 8 to11years old.

The PASES was chosen for this study because other physical activity self-efficacy scales do not distinguish adult physical activity self-efficacy from children physical activity self-efficacy (Bartholomew, Jowers, Loukas, \& Allua, 2005; Kroll, Kehn, Ho, \& Groah, 2007). In the PASES, while most barrier items were generated from adult physical activity self-efficacy, the positive alternatives items were specific to children (Saunders et al., 1997). Additionally, this scale is suitable and understandable for children aged 8 to 11years old.

Physical activity enjoyment. The Physical Activity Enjoyment Scale (PACES) (See Appendix H) is a 16-item self-report instrument that assesses children's positive affect associated with participation in physical activity. It is a five-point Likert scale $(1=$ disagree a lot, to $5=$ agree a lot) with higher score indicating higher levels of physical activity enjoyment (Moore et al., 2009). This scale was first developed by Kendzierski and colleagues in 1991 to assess adults' extent of enjoyment in doing any given physical activity. The original PACES included 18 items containing bipolar statements (I enjoy it vs. I hate it) with 7 points between each statement; Cronbach's alpha coefficient was .93 in college students. A study among 279 adolescents aged 12 to17 years old indicated that the original 18-item scale had adequate internal consistency but inadequate construct validity (Crocker, Bouffard, \& Gessaroli, 1995). 
In 2001, Motl and colleagues modified the PACES to be used among adolescent girls. They deleted two items - Item 5 (I am very absorbed in the activity), and Item 11 (It's very invigorating) because their content were not relevant to physical activity enjoyment in adolescents or were redundant. Moreover, the scale was changed from a 7point bipolar scale to a more easily understood 5-point Likert scale. Later, a study was designed to assess the validity of the revised PACES in 1,797 adolescent girls. The results indicated that the 16-item PACES was a valid measurement to assess physical activity enjoyment among adolescent girls (Motl et al., 2001).

In 2009, Moore and colleagues tested the 16-item PACES in 564 third grade children. After obtaining students' feedback of their understanding of the items, the word "depressed" was changed to "sad". Results indicated that the scale had acceptable internal consistency with Cronbach's alpha coefficient of .87 for the entire sample, .87 for European American boys, .88 for European American girls, .86 for African American boys, and .87 for African American girls. Moreover, physical activity enjoyment was significantly correlated with task goal orientation, perceptions of athletic competence, physical appearance, and self-reported physical activity indicating this scale had acceptable convergent validity. When analyzing the scale, items $1,4,6,8,9,10,11,14$, and 15 were reverse coded as 5 to 1 (Moore et al., 2009). In the feasibility study among 24 children aged 8 to 13 years old, the Cronbach's alpha coefficient was .89 (Ling, 2012). Thus, the 16-item PACES appears to be a reliable and valid scale used in children to assess physical activity enjoyment. 


\section{Reinforcing Factor}

Parental influence. The Parental Influence Scale (PAIS) (See Appendix I) is an 18-item scale with four components - parental involvement, parental facilitation, parental encouragement and parental role modeling. The PAIS uses a 4-point Likert scale with higher score indicating higher levels of parental influence. Items for parental role modeling and parental encouragement were generated from two previously validated scales (Brustad, 1993). Items for parental facilitation and parental involvement were created by Welk and colleagues (Welk et al., 2003).

Originally, Welk thought the PAIS scale should load into four factors, but the exploratory factor analysis suggested a two-factor structure with parental encouragement, involvement, and facilitation becoming one factor - parental support. A study in 994 children in grades 3-6 found that this scale had acceptable internal consistency with Cronbach's alpha coefficient of .81. Scores on the scale accounted for $19.7 \%, 25.8 \%$, and $28.3 \%$ of the variance in self-reported physical activity, attraction to physical activity, and perception of competence respectively suggesting the scale had acceptable predictive validity. Similarly, after splitting the subjects into two groups based on the levels of parental influence, the significant effect of parental influence on self-reported physical activity, attraction to physical activity, and perception of competence indicated that the scale had acceptable discriminant validity (Welk et al., 2003).

The bipolar statements (Some kids have parents who get a lot of exercise vs. Other kids have parents who don't get a lot of exercise) in the PAIS were found to be confusing for children, thus, the bipolar statements were changed to unipolar statements following King's suggestion (2008). In one study among 176 adolescents using unipolar 
statements, the Cronbach's alpha was .79 for the parental role modeling subscale, and .89 for the parental support subscale, indicating acceptable internal consistency (King, Ogletree, Fetro, Brown, \& Partridge, 2011). When analyzing the scale, four items (some kids have parents that don't help them much with sports, don't take them to parks or playgrounds, don't encourage them to play outside, or don't like to do much physical activity) were reverse coded as 4 to 1 . In the feasibility study among 24 children aged 8 to 13-years old, the Cronbach's alpha coefficient was .89 for the modified scale with unipolar statements (Ling, 2012). In all, the modified PAIS has acceptable reliability to assess parental influence among children and was used to operationalize parental influence for the study.

\section{Enabling Factor}

Environment. No existing scale developed to assess school environment for children was identified, thus the Measure of Perceived Environment was modified, adding one item to measure school environment. The modified Measure of Perceived Environment was used to assess the environmental determinants of physical activity among children in this study (See Appendix J).

The original four item Measure of Perceived Environment was developed by Motl and colleagues (2005) among 633 adolescent girls. Results from confirmatory factor analysis confirmed a two-factor structure - equipment accessibility (Item 1 and 2) and neighborhood safety (Item 3 and 4). The two-factor structure was consistent across time and between African and Caucasian girls (Motl et al., 2005). Another study in 49 children and adolescents aged 11 to 14 years old found that the neighborhood safety subscale had a Cronbach's alpha coefficient of .62 (King, 2008). Moreover, items 1, 2, 3 and 4 had 
significant test-retest correlation coefficients of $.35, .64, .43$ and .42 , respectively, indicating acceptable stability over time (King, 2008).

The equipment accessibility subscale included only home and community components. For children who spend most of their time in school, school environment is an indispensable component that influences children's physical activity levels. Thus, Item 5 'At school, there are playgrounds, gym spaces, and enough supplies (like balls, hula hoops) to use for physical activity' was added to address the school component of equipment accessibility. The four original items and the additional item added to address the school environment were scored from 1 (Disagree a lot) to 5 (Agree a lot); item 4 was reverse scored before analysis. The total score of the five items was used to describe the perceived environment with higher score indicating a more supportive environment for physical activity.

\section{Summary}

The above instruments were carefully chosen and evaluated to determine their appropriateness for use with children aged 8 to 11 years old in this study, and their reliability and validity to accurately and consistently measure the study variables. Study variables and instruments used in this study are summarized in Table 4. 


\section{Table 4}

$\underline{\text { Instruments to Operationalize Variables }}$

\begin{tabular}{|c|c|c|c|c|}
\hline Variable & Instrument & Number of Items & Dimensionality & Psychometric Properties \\
\hline $\begin{array}{l}\text { Personal } \\
\text { Demographics }\end{array}$ & $\begin{array}{c}\text { Demographic information } \\
\text { questionnaire }\end{array}$ & 14 & - & - \\
\hline Obesity (BMI) & {$\left[(\right.$ weight $\left.\mathrm{kg}) /(\text { height } m)^{2}\right]$} & 2 & - & - \\
\hline Physical Activity & $\begin{array}{l}\text { Pedometer Logs } \\
\text { PAQ-C }\end{array}$ & 9 & One & $\begin{array}{l}\text { - Cronbach's alpha: } .82 \\
\text { - Test-retest reliability: } .79 \\
\text { - Convergent validity: } r=.28-.63 \\
\text { - Cronbach's alpha (feasibility): } .86\end{array}$ \\
\hline $\begin{array}{l}\text { Physical Activity } \\
\text { Self-efficacy }\end{array}$ & PASES & 17 & Three & $\begin{array}{l}\text { - } \text { Cronbach's alpha: .68 } \\
\text { - } \text { Test-retest reliability: .75 } \\
\text { - } \text { Cronbach's alpha (feasibility): .79 }\end{array}$ \\
\hline $\begin{array}{l}\text { Physical Activity } \\
\text { Enjoyment }\end{array}$ & PACES & 16 & One & $\begin{array}{l}\text { - Cronbach's alpha: .86-.88 } \\
\text { - Cronbach's alpha (feasibility): } .89\end{array}$ \\
\hline Parental Influence & PAIS & 18 & Two & $\begin{array}{l}\text { - Cronbach's alpha: .79-.89 } \\
\text { - Cronbach's alpha (feasibility): .89 }\end{array}$ \\
\hline Environment & $\begin{array}{l}\text { Modified Measure of } \\
\text { Perceived Environment }\end{array}$ & 5 & Two & $\begin{array}{l}\text { Motl's Perceived Environment Scale } \\
\text { - Cronbach's alpha: .62 } \\
\text { - Test-retest reliability: } r=.35-.64\end{array}$ \\
\hline
\end{tabular}




\section{Data Collection}

Before data collection, approval from the University Institutional Review Board (IRB) was obtained. Subsequently, the investigator visited the selected ten elementary schools (two schools at a time) with afterschool programs to explain the study purposes and procedures from August 2013 to October 2013. All eligible children were assigned a discreet identifier, a number which was only used to match children's information on the demographic questionnaire, pedometer logs, and self-reported surveys. A total of 146 eligible children received a recruitment folder containing a letter describing the voluntary nature of the research study, a demographic information questionnaire, and the informed consent document approved by the University IRB. Children were instructed to take the folder home to their parents or legal guardians to review. A telephone number and an email address for the investigator were provided so that parents or legal guardians had an opportunity to discuss the study if desired. The recruitment folders were returned by the children to their teacher or program director and held for the research staff, or given directly to the research staff in the data collection setting. Teachers or program directors were asked to remind children to bring the recruitment folder back. In addition, the study investigator talked with each parent or legal guardian about this study when they picked up their children from the afterschool programs. After one week, children who had not returned a recruitment folder were provided with a second recruitment folder, containing the same documents, which were sent home for parents or legal guardians to review. If no consent was obtained one week after the second recruitment folder was distributed, the parent/child was considered a non-responder and that child was not enrolled in the study. Six eligible children ( 3 girls and 3 boys) did not participate in this study due to lack of 
parental consent. To increase the likelihood that children would return the consent form and demographic instrument, all children who brought back the informed consent form, regardless of parents/guardians agreement, were included in a drawing for a basketball. After obtaining the informed consent from parents/guardians, the investigator explained all study procedures to children, and the assent form approved by the University IRB was obtained from children. If a child did not assent to participate in the study, then data was not collected from him/her. Seven children (5 girls and 2 boys) did not assent to participate in this study due to lack of fun, "challenge of wearing pedometers", or did not want to complete the surveys. After obtaining the informed consent from parents/guardians and the assent from children, the investigator measured all children's height and weight one at a time, in a private room/place after school, and recorded the information in both the pedometer logs (one for investigator and one for child) to maintain information. The reason for measuring children's weight and height directly, rather than using parents' reports of height and weight was that in the feasibility study the majority of parents did not know their child's height and weight (Ling, 2012). The processes for measuring height and weight, as described below, were standardized according to the guidelines published by the CDC for measuring a child's height and weight at home (CDC, 2011d).

Children's height was measured by the investigator, to the nearest tenth of a centimeter using the Seca 213 Portable Stadiometer (Seca North America, CA, USA) (CDC, 2011d). When measuring a child's height, the child was asked to remove his/her shoes, and stand with feet together, flat on the floor, with the child's head, shoulders, buttocks and heels touching the Stadiometer, with the flat headpiece forming a right angle 
with the wall and firmly touching the crown of the child's head. Then the investigator recorded the child's height to the nearest .10 centimeter on the two pedometer logs (CDC, 2011d). Weight was assessed to the nearest tenth of a kilogram using the Tanita HD-351 Scale (Tanita Corp., Illinois, USA). When measuring a child's weight, the child was asked to remove his/her shoes and heavy clothes such as jackets or sweaters, and place both feet flat on the center of the scale with hands placed over his/her belly button. Then the investigator recorded the child's weight to the nearest .10 kilograms on the two pedometer logs (CDC, 2011d).

After measuring a child's height and weight, the investigator distributed the pedometers and pedometer logs to him/her, and reviewed detailed instructions with the child. Each child was then asked to attach the pedometer to the waistband of his/her clothing in the morning from Day 1 to Day 7, and was instructed to keep the pedometer dry and leave the pedometer turned on until he/she went to bed. To improve the compliance rate, every school day afternoon, the investigator visited the afterschool programs to help children record the step counts on two pedometer logs, reset pedometers to zero after recording steps, and remind children to wear the pedometers if they had forgotten. On the weekend, children recorded the step counts on the pedometer logs with the help of their parents/guardians. Seven days later, children were asked to return the pedometers and pedometer logs to the investigator or school staffs on Day 8 or the next school day.

After obtaining pedometers and pedometer logs, children were asked to complete the posttest surveys in a private room/place, with the investigator sitting with each child reading the questions aloud to ensure the children understand the items on each 
questionnaire. Finally, the investigator checked the questionnaires for missing data. Some children had difficulty in understanding some types of physical activity, thus a small card with a picture describing the type of physical activity, developed in the feasibility study (Ling, 2012), was shown to children to help them understand. Due to parents' schedules, some children were not able to finish the survey in a single session; a second appointment was scheduled with the child after school to finish the remainder of the questions if needed. For children who completed the whole study, a further incentive gift (a playground ball) was awarded.

In addition to the above procedures, the 50 randomly selected children participating in the pretest-posttest study were asked to complete the pretest surveys assessing physical activity self-efficacy, physical activity enjoyment, parental influence and environment on the day they signed the assent and before they received pedometers. Children completed the pretest surveys in a private room/place, with the investigator sitting with each child reading the questions to ensure that children understand the items on each questionnaire. Finally, the investigator checked the questionnaires for missing data. Once data collection was over, the investigator placed all the informed consent forms in a box, drew five forms from the box, and awarded basketballs as prizes to the five winners.

\section{Data Analysis}

\section{Data Cleaning}

Several strategies were used to identify erroneous observations. First, all entry cells were programmed to detect inconsistent and invalid data including invalid codes and values that were out of range. After data entry, summary (frequency table, minimum and 
maximum values) and graphic techniques (boxplot, histogram, and scatterplot) were used to identify problem values and outliers. All the identified problem values and outliers were checked with the original forms.

\section{Instruments Coding}

Coding of the instrument PAQ-C followed the published instructions for scoring that instrument (See Table 5) (Kowalski, Crocker, \& Donen, 2004). The mean score of the pedometer steps was used to describe children's objective physical activity levels, and the total score of the PAQ-C was used to describe the self-reported physical activity levels, with a score of 1 indicating low levels of physical activity, whereas a score of 5 indicating high levels of physical activity (Kowalski et al., 2004).

\section{Table 5}

$\underline{\text { Steps for Coding the PAQ-C }}$

\begin{tabular}{|c|c|}
\hline Items & Coding Methods \\
\hline Item 1 & $\begin{array}{l}\text { - Code "no" to } 1 \text {, and "7 times or more" to } 5 \text {. } \\
\text { - Take the mean of all activities to form a composite score. }\end{array}$ \\
\hline Items 2 to 8 & - Code the answers for each item from 1 to 5 . \\
\hline Item 9 & $\begin{array}{l}\text { - Code "none" to } 1 \text {, and "very often" to } 5 \text {. } \\
\text { - Take the mean of all days of the week to form a composite score. }\end{array}$ \\
\hline Total Score & - Take the mean of the above 9 items. \\
\hline
\end{tabular}

For the PASES, items 1, 2, 5, 7, 8, 9 and 12 describe the support seeking component; items 6,10,13 and 14 describe the barriers component; and items 3, 4, 11, 15, 16 and 17 describe the positive alternatives component. The total score for each component was calculated and the total score for the 17 items was used to describe physical activity self-efficacy. The total score of the 16 stems of the PACES was used to 
describe physical activity enjoyment. For the PAIS, items 1, 4, 11, 13, 15 and 17 describe parental role modeling component, while the rest of items describe parental support component. The total score of the scale was calculated to describe perceived parental influence. Moreover, total score for the five items of the modified environment scale was used to describe environmental determinants of physical activity.

\section{Reliability and Validity Checks}

The Cronbach's alpha technique and item-total correlation coefficients were utilized to assess the internal consistency reliability of the PASES, PACES, PAIS, environment scale and PAQ-C among children. In addition, intraclass correlation coefficient (ICC) was estimated by a two-way mixed model (people effect is random, and time effect is fixed) with type consistency (systemic differences between two tests are considered irrelevant) - ICC $(2,2)$ model (McGraw \& Wong, 1996; Weir, 2005) - to assess the test-retest reliability of the PASES, PACES, PAIS and environment scale if wearing pedometers did not have significant influence on the hypothesized determinants of physical activity. Usually, a scale with ICC $<.40$ has poor reliability, a scale with .40 $\leq \mathrm{ICC}<.75$ has fair to good reliability, and a scale with ICC $\geq .75$ has excellent reliability (Zaki, Bulgiba, Nordin, \& Azina-Ismail, 2013). The Pearson correlation coefficient between the score of the PAQ-C and pedometer steps was used to assess the convergent validity of the PAQ-C. Confirmatory factor analysis was used to assess the construct validity of the PASES, PACES and PAIS. Additionally, exploratory factor analysis was used to explore the factorial structure of the environment scale. 


\section{Assumptions of Structural Equation Modeling}

To use structural equation modeling (SEM), four assumptions need to hold: (1) absence of univariate and multivariate outliers; (2) multivariate normality: all the variables (self-efficacy, enjoyment, parental influence, environment and physical activity) follow normal distribution with respect to each other; (3) the relationships among variables are linear; and (4) absence of multicollinearity or singularity: the variables are very high correlated $(r>.70$, multicollinearity), or the variables are redundant (singularity) (Tabachnick \& Fidell, 2007).

Outliers. Univariate outliers are cases with an extreme value on one variable, while multivariate outliers are cases with an unusual combination of scores on two or more variables. To find univariate outliers, frequency table was used for categorical variables, while histograms and box plots were used for continuous variables. No univariate outlier was identified for the data. Multivariate outliers were identified by examining Mahalanobis distance, Cook's distance, and leverage values for each individual. Mahalanobis distance was evaluated as $x^{2}$ with degree of freedom equal to the number of variables (in this study, $d f=5$ ) in the analysis. If a participant has a Mahalanobis distance greater than $x^{2}(5)=20.515$, then he/she is considered as a multivariate outlier. A conventional cut off point for Cook's distance is $4 / n$, where $n$ is the number of observations; while a cut off value for leverage is $2 p / n$, where $p$ is the number of parameters. In this study, a participant was considered as a multivariate outlier when he/she had Mahalanobis distance $>20.515$, Cook's distance $>4 / n=.03$, and leverage value $>2 p / n=.19$ (Tabachnick \& Fidell, 2007). Although two cases had 
Mahalanobis distance greater than 20.515, their Cook's D and leverage values were less than the cut off points. In other words, no influencing outlier was identified from the data.

Multivariate normality. Multivariate normality was evaluated using Mardia's test for multivariate normality. For the self-reported physical activity models, the Mardia's multivariate Kurtosis ranged from 7.58 to 9.78, suggesting absence of multivariate kurtosis (Gao, Mokhtarian, \& Johnston, 2008). For the pedometer steps models, Mardia's multivariate Kurtosis ranged from 6.84 to 8.52 , suggesting absence of multivariate kurtosis. In addition, univariate indices of skewness and kurtosis were examined using SPSS FREQUENCIES to determine if the absolute value of skewness was greater than 2.0, or the kurtosis absolute value was greater than 7.0 (West, Finch, \& Curran, 1995). Skewness can affect the means of the tests, while kurtosis can affect the variance and covariance of the tests. All the variables' skewness and kurtosis absolute values were less than 1.0 in this study.

Linearity. Bivariate scatterplots were used to evaluate the relationship between one independent variable and dependent variable. If the scatterplot is oval-shaped, then the assumption of linearity holds (Tabachnick \& Fidell, 2007). If the scatterplot is not oval-shaped, then variable transformation (such as square root, logarithm or inverse) will be used. All the scatterplots between each two variables in this study were oval-shaped or very close to oval-shaped, indicating linear relationship between each two variables.

Multicollinearity and singularity. Multicollinearity occurs when variables are strongly correlated $(r>.70)$. The correlation matrix was used to evaluate multicollinearity (Tabachnick \& Fidell, 2007). Singularity occurs when variables are redundant, that is, one variable is a combination of two or more of the other variables. 
Moreover, tolerance values for each independent variable were used to detect multicollinearity. Tolerance is the variance of one independent variable that is not explained by other independent variables. In the matrix Table 13, all the correlation coefficients between each two hypothesized determinants were less than .70, and the tolerance values for all the independent variables were greater than .10 ranging from .58 to .77 . Thus, the multicollinearity is not a problem for this data set.

\section{Preliminary Analysis}

Descriptive statistics were utilized to describe the demographic characteristics of children. Continuous variables were described by Mean $(M)$ and Standard Deviation $(S D)$, while categorical variables were described by frequency and percentage. In addition, descriptive statistics ( $M$ and $S D$ ) were used to describe the data on physical activity selfefficacy using the PASES, physical activity enjoyment using the PACES, perceived parental influence using the PAIS, environment using the environment scale, and physical activity levels using the PAQ-C and pedometer steps among children aged 8 to 11 years old.

When the demographic variable was categorical, chi-square test was used to test the influence of demographics on obesity status. Furthermore, a logistic regression model was applied to assess the influence of physical activity and sedentary behavior on obesity status (overweight/obesity $=1$, or non-overweight $=0$ ) after controlling for other demographics. Two-sample independent t-test was utilized to test sex differences on physical activity self-efficacy, physical activity enjoyment, parental influence, environment and physical activity, while simple linear regression was used to explore the influence of age and children number on physical activity self-efficacy, physical activity 
enjoyment, parental influence, environment and physical activity. A linear regression was conducted with dependent variable PAQ-C score and independent variable pedometer steps. Moreover, a scatterplot was drawn to see what cases are outliers.

One-way ANOVA was applied to test other demographic differences on physical activity self-efficacy, physical activity enjoyment, parental influence, environment and physical activity. If significance $(p<.05)$ was identified from the ANOVA test, then different contrast tests were applied based on group sample sizes and variance: (1) R-EG-W-Q or Tukey post-hoc test was used when groups had equal sample size and homogenous variance; (2) Gabriel's post-hoc test was used when group sample sizes were slightly different; (3) Hochberg's GT2 post-hoc test was used when group sample sizes were very different; and (4) Games-Howell post-hoc test was used when the assumption of homogeneity of variance was violated. Cochran-MantelHaenszel Statistics was used when the dependent variables were the percentage of children meeting daily physical activity recommendations. Paired-samples t-test was used to assess the differences of physical activity self-efficacy, physical activity enjoyment, parental influence and environment between pretest and posttest among the randomly selected 50 children.

\section{Primary Analysis}

Structural Equation Modeling (SEM) - path analysis, which is used to test relationships among manifest variables (directly measured), was used to develop the paths among physical activity self-efficacy, physical activity enjoyment, parental influence, environment and physical activity (one model with self-reported physical activity levels, and a second model with pedometer steps) using the SAS 9.3 for 
Windows (SAS Institute Inc, Cary, NC). For the self-reported physical activity models, maximum likelihood (MI) method was used because six children did not complete any of the posttest surveys, while full information maximum likelihood (FIML) method was used for the pedometer steps models because three out of the six children wore pedometers for at least one day. A variety of global fit indices were used to evaluate model fit, including indices of absolute fit, indices of incremental/relative fit, and indices of fit with a penalty function for lack of parsimony. Absolute fit indices, including chisquare statistic, Goodness-of-Fit Index (GFI), Root Mean Square Residual (RMSR), standardized RMSR (SRMSR), and Hoelter Critical N index (CN), determine how well a proposed model fits the observed covariance matrix. Model parsimony indices, including Adjusted Goodness-of-Fit Index (AGFI), Parsimony Goodness-of-Fit Index (PGFI), Root Mean Square Error of Approximation (RMSEA), Akaike's Information Criterion (AIC), Schwarz Bayesian Criterion (SBC), Parsimony Normal Fit Index (PNFI), and Parsimony Comparative Fit Index (PCFI), evaluate model fit by taking into account the complexity of a model. Incremental fit indices, including Normal Fit Index (NFI), Tucker-Lewis Index (TLI), Relative Noncentrality Index (RNI), and Comparative Fit Index (CFI), evaluate the proportionate improvement in fit by comparing a proposed model with a baseline model.

In this study, the minimum discrepancy (CMIN), is most commonly expressed as a chi-square statistic $x^{2}$, Hoelter CN, GFI and SRMSR were used to assess the absolute fit. The CMIN follows a chi-square distribution with degree of freedom equaling to $\frac{1}{2 p(p+1)}-t$, where $p$ is the number of observed variables, and $t$ is the number of parameters to be estimated (Hu \& Bentler, 1999). A model with chi-square test non- 
significant $(p>.05)$ indicates absolute fit. Hoelter CN estimates the largest sample size that can make chi-square model fit statistic still non-significant. The $\mathrm{CN}$ value should be at least 200 indicating that the study has adequate sample size for using the SEM (Hoelter, 1983). The GFI is a measure of the relative amount of variance and covariance in the study sample that is jointed explained by the population variance-covariance matrix. A model with a GFI value greater than .90 indicates goodness of fit. The SRMSR is the average residual value between variance-covariance matrix of the hypothesized model and variance-covariance matrix of the sample data. A model with a SRMSR value less than .05 has good fit (Hu \& Bentler, 1999).

The AGFI and RMSEA were used to assess model parsimony. Compared to GFI, AGFI takes into account of the number of parameters into the assessment of model fit. A model with an AGFI value greater than .95 has good fit. The RMSEA value is related to the residual of a model with smaller value indicating better model fit. A model with an RMSEA value of .06 or less, and $p$-value for closeness of fit test greater than .05 has good fit (Hu \& Bentler, 1999). The CFI was used to evaluate incremental fit. The difference between NFI and CFI is that CFI takes sample size into account. A model with a CFI value greater than .95 has good model fit (Hu \& Bentler, 1999).

If the model fit was acceptable, then the parameters were estimated. The ratio of each parameter estimate to its standard error follows $z$ distribution and is significant at $\alpha$ level of .05 when the ratio is greater than 1.96. There are two kinds of parameter estimates: unstandardized parameter estimates and standardized parameter estimates. Unstandardized parameter estimates retain scaling information of variables and can only be interpreted with reference to the scales of the variables; while standardized parameter 
estimates, corresponding to effect-size estimates, remove scaling information and can be used for informal comparisons among parameters of the model (Suhr, 2012). A standardized path coefficient with absolute value less than .10 indicates a small effect, value ranging from .10 to .50 indicates a medium effect, while value greater than .50 indicates a large effect (Suhr, 2012).

If unacceptable model fit was found, then the model was modified to get a betterfit model. Non-significant paths were eliminated one at a time based on their path coefficients, that is, non-significant path with the smallest path coefficient was eliminated first. Model fit was evaluated for all the models, and Akaike's Information Criterion (AIC) and Expected Cross-Validation Index (ECVI) were used to compare the goodnessof-fit among self-reported physical activity models, while AIC and Schwarz Bayesian Criterion (SBC) were used to compare model fit for pedometer steps models. The AIC can assess the relative information lost when using a model to estimate reality. The ECVI measures the discrepancy between the fitted covariance matrix in the hypothesized path model and the expected covariance matrix that will be obtained in another sample of equivalent size from the same population (Byrne, 2010). The SBC is very similar to AIC. When using FIML method, SBC takes the consideration of incomplete observations. The model with the smallest AIC value, ECVI value or SBC value has better fit, and was selected as the final model.

\section{Protection of Human Subjects}

One hundred and thirty-three children aged 8 to 11 years old were recruited from ten elementary schools with afterschool programs in the CISCC. Prior to data collection, IRB approval was obtained, then the parental informed consent form approved by the 
University IRB was distributed to children's parents or legal guardians through children. The parental informed consent form outlined study purposes, procedures, nature of the commitment, participation selection, potential risks and benefits, participation gifts (basketball prize and a playground ball), confidentiality pledge, voluntary consent, right to withdraw at any time, and the contact information of the investigators. After receiving the signed consent forms from parents/guardians, the investigator explained to the selected children the study purposes, procedures, risks and benefits, and informed them of their right to decline to participate and to withdraw from the study at any time. Then the assent form approved by the University IRB was obtained from the children. The investigator was available physically or by telephone/email to answer all questions the children or parents/guardians might ask prior to the children's participation and at any time during the course of the study.

All parents or legal guardians were asked to complete an instrument assessing demographic characteristics. Children willing to participate in this study were asked to wear pedometers for seven consecutive days, record pedometer steps in the pedometer logs daily with the help of investigator or parents/guardians, and complete a series of standardized posttest surveys assessing physical activity self-efficacy, physical activity enjoyment, parental influence, environment and self-reported physical activity levels on the eighth day. The 50 randomly selected children completed the pretest surveys assessing physical activity self-efficacy, physical activity enjoyment, parental influence and environment before they received pedometers. All the data were collected solely for research purposes and results were reported only in aggregate form. 
The study participants for this study were children, so special consideration was taken to protect children's rights and confidentiality, as well as to minimize the potential for emotional distress. Children aged 8 to 11 years old share a number of unique developmental characteristics: (1) children below 13 years old do not have the competence to give informed consent; and (2) children above 7 years old have the competence and the right to give assent (Polit \& Beck, 2004). Therefore, assent was obtained from children and informed consent was obtained from their parents or legal guardians. To minimize the potential for embarrassment, harassment or other emotional distress, children's weight and height were measured one at a time in a private room/place, and children completed the surveys one at a time with the help of investigator in a private room/place separating from other children.

Every precaution was taken to minimize risks of participating in this study by ensuring children's safety in the data collection setting and their right to privacy. Confidentiality for all children was ensured by identifying data collection instruments using discreet identifiers instead of children's names, and keeping all questionnaires in locked cabinets and recording data only in restricted secure university computer account. The study placed children at minimal risks considering the study design, data collection method and study settings.

\section{Summary}

This study aimed to explore the psychosocial and environmental determinants of physical activity through examining the relationships among physical activity selfefficacy, physical activity enjoyment, parental influence, environment and physical activity among children aged 8 to 11 years old. To clarify the relationships among 
physical activity self-efficacy, physical activity enjoyment, parental influence, environment and physical activity, a cross-sectional correlational research methodology was used. Moreover, a pretest-posttest study was conducted among a subsample of 50 randomly selected children to examine the influence of wearing pedometers on children's physical activity self-efficacy, physical activity enjoyment, parental influence and environment. Based on the statistical method of SEM used in this study, a total of 133 children were recruited from ten elementary schools with afterschool programs to obtain a representative and diverse sample.

The study investigator measured each child's height and weight in school. All the children received a pedometer for wearing for seven consecutive days, and were asked to complete the posttest surveys measuring physical activity, physical activity self-efficacy, physical activity enjoyment, parental influence and environment after they returned the pedometers and pedometer logs. The 50 randomly selected children needed to complete the pretest surveys measuring their physical activity self-efficacy, physical activity enjoyment, parental influence and environment before receiving a pedometer.

One hundred and thirty-three children out of 146 eligible children participated in this study, and six children (one in the pretest-posttest study) did not complete the posttest surveys due to a health problem, injury or family relocation to a different geographical area. Among the 133 children, approximately 20\% were Hispanics, 26\% were African Americans, and 57\% were living with their both parents. About 18\% of the fathers and $15 \%$ of the mothers were unemployed, and $50 \%$ fathers and $43 \%$ mothers did not receive any college education. 


\section{CHAPTER IV}

\section{RESULTS}

This chapter presents the research findings from this study. First, scale construction and psychometric characteristics for all the self-reported scales are evaluated, followed by the compliance rate, reliability and reactivity of wearing pedometers among elementary school children attending afterschool programs. Then the descriptions of sedentary activity, physical activity, obesity status, the hypothesized determinants of physical activity, and their relationships with demographics are presented. Finally, the major findings related to the four research questions are addressed respectively. A summary of the key findings is provided at the end.

\section{Scale Construction and Psychometric Characteristics}

\section{Reliability}

Internal consistency reliability. The Cronbach's alpha coefficient was .88 for the 9-item PAQ-C, with item-total correlation coefficients ranging from .10 to .47. The items with small item-total correlation coefficients $(<.20)$ were 'question 1 : Rowing/canoeing', and 'question 3: what did you do most of the time at recess?' This may be due to that some children in this study complained that they did not have recess during school time, and they had few opportunities for rowing/canoeing considering their parents' interests, socioeconomic levels and employment status. The Cronbach's alpha coefficient was .73 for the 17-item PASES, with item-total correlation coefficients ranging from .15 to .48 . The items with small item-total correlation coefficients $(<.20)$ 
were 'question 1: I think I can be physically active most days after school', and 'question 3: I think I can be physically active after school even if I could watch TV or play video games instead'. The 16-item PACES had Cronbach's alpha coefficient of .83, with itemtotal correlation coefficients ranging from .25 to .65 , indicating good internal consistency reliability. The Cronbach's alpha coefficient was .82 for the 18 -item PAIS, with itemtotal correlation coefficients ranging from .15 to .61 . The only item with small item-total correlation coefficient $(<.20)$ was 'My parents tell me not to watch too much TV'. This may be explained by the non-significant correlation between physical activity and screenviewing time. In other words, parental discouragement of watching TV may be not strongly correlated with parental influence on physical activity. On the contrary, parents usually hesitate to discourage children from watching TV because this may cause conflict in the home due to children's anger at this rule or increased bickering among siblings, or because TV is considered offering entertainment, free babysitting and educational opportunities for children (Evans, Jordan, \& Horner, 2011). The modified environment scale had a Cronbach's alpha coefficient of .51, with item-total correlation coefficients ranging from .17 to .37 , which is acceptable considering its small number of items. The only item with small item-total correlation coefficient $(<.20)$ was 'It is difficult to walk or jog in my neighborhood because of things like traffic, no sidewalks, dogs, or gangs'. During data collection, the investigator found that children had difficulty understanding the negatively worded questions.

Test-retest reliability. Because no significant differences were found on the determinants of physical activity after one-week wearing pedometers, a two factor mixed effects model with type consistency was used to calculate intraclass correlation 
coefficient (ICC) to evaluate the test-retest reliability of the PASES, PACES, PAIS and modified environment scale. The ICC for the PASES was .83 with $95 \%$ confidence interval (CI) $[.69, .90]$; the ICC for the PACES was .63 with 95\% CI $[.35, .79]$; the ICC for the PAIS was .84 with 95\% CI $[.69, .90]$; and the ICC for the modified environment scale was .61 with 95\% CI [.30, .78]. Therefore, all the self-reported scales have acceptable test-retest reliability (Zaki et al., 2013). 


\section{Table 6}

$\underline{\text { Psychometric Properties of the Major Study Variables }(N=133)}$

\begin{tabular}{|c|c|c|c|c|c|c|c|c|}
\hline \multirow[b]{2}{*}{ Scale } & \multirow[b]{2}{*}{$M$} & \multirow[b]{2}{*}{$S D$} & \multicolumn{2}{|c|}{ Reliability } & \multicolumn{2}{|c|}{ Range } & \multirow[b]{2}{*}{ Skewness } & \multirow[b]{2}{*}{ Kurtosis } \\
\hline & & & Cronbach's $\alpha$ & $I C C$ & Potential & Actual & & \\
\hline PAQ-C & 3.20 & .70 & .88 & - & $1-5$ & $1.32-4.64$ & -.36 & -.08 \\
\hline PACES & 66.87 & 9.28 & .83 & .63 & $16-80$ & $40-80$ & -.71 & .23 \\
\hline PASES & 13.45 & 2.95 & .73 & .83 & $0-17$ & $2-17$ & -.91 & .84 \\
\hline Support Seeking & 6.00 & 1.33 & .61 & .73 & $0-7$ & $0-7$ & -1.43 & 2.32 \\
\hline Barriers & 2.87 & 1.25 & .64 & .86 & $0-4$ & $0-4$ & -.78 & -.59 \\
\hline Positive Alternatives & 4.57 & 1.29 & .44 & .75 & $0-6$ & $1-6$ & -.57 & -.46 \\
\hline PAIS & 52.75 & 8.92 & .82 & .84 & $18-72$ & $28-72$ & -.03 & -.07 \\
\hline Parental Support & 36.02 & 6.25 & .76 & .74 & $12-48$ & $17-48$ & -.32 & .26 \\
\hline Role Modeling & 16.74 & 3.75 & .68 & .81 & $6-24$ & $6-24$ & .01 & -.12 \\
\hline Environment & 18.30 & 3.80 & .51 & .61 & $5-25$ & $5-25$ & -.37 & .58 \\
\hline Equipment Access & 11.80 & 2.77 & .66 & .53 & $3-15$ & $3-15$ & -1.06 & 1.01 \\
\hline Neighborhood Safety & 6.50 & 2.38 & .49 & .60 & $2-10$ & $2-10$ & -.27 & -.74 \\
\hline
\end{tabular}




\section{Validity}

Factorial structure of the physical activity self-efficacy scale. A confirmatory factor analysis (CFA) was conducted to test the factorial structure of the PASES. Results of the CFA model indicated that the three-factor structure of the PASES did not fit the data well with fit indices $\left[x^{2(116)}=160.55, p=.0039, \mathrm{CN}=112, \mathrm{SRMSR}=.08, \mathrm{GFI}\right.$ $=.89, \mathrm{AGFI}=.85, \mathrm{RMSEA}=.06$ with $90 \% \mathrm{CI}[.03-.07], \mathrm{CFI}=.82, \mathrm{NFI}=.58, \mathrm{ECVI}=$ $1.96, \mathrm{AIC}=234.55]$. Thus, an exploratory factors analysis with Varimax rotation was conducted to assess the factorial structure of the PASES. The results of the Bartlett's test indicated that there were significant correlations among the 17 items $\left[x^{2}(136)=362.04, p\right.$ $<.0001]$, and the Kaiser-Meyer-Olkin (KMO) measures of sampling adequacy was .64, indicating that the factor analysis can yield distinct and reliable factors (Kaiser, 1974). However, no satisfactory factorial structure was identified based on the Scree plot, eigenvalues, variance explained, and loading coefficients. The content of Item 16 is very similar to Item 4, and Item 17 is very similar to Item 1. Based on the item-total correlation coefficients, Item 1 with item-total correlation coefficient of .15 and Item 4 with item-total correlation coefficient of .27 were deleted to form a new physical activity self-efficacy scale: physical activity self-efficacy scale for children (PASES-C). The original 17-item PASES and 15-item PASES-C were highly correlated with each other $(r$ $=.99, p<.0001)$. The Cronbach's alpha coefficient for the PASES-C was .72 with itemtotal correlation coefficients ranging from .14 to .49 , with Item 3 having the item-total correlation coefficient of .14.

The Bartlett's test for the 15 items were significant $\left(x^{2}=306.02, d f=105, p\right.$ $<.0001$ ), and the KMO measures of sampling adequacy was .68. A principle components 
analysis with Varimax rotation suggested a new three-factor structure - barriers, support seeking, and positive alternatives. If one item loaded onto two factors, then that item would be assigned to the component with a bigger loading coefficient. Item 8 was assigned to support seeking component after reading the content of that item (See Table 7). The three factors accounted for $41.08 \%$ variance of the physical activity self-efficacy. The CFA model suggested that the three-factor structure of the PASES-C still did not fit the data adequately $\left[x^{2}(87)=114.62, p=.0253, \mathrm{CN}=121, \mathrm{SRMSR}=.08, \mathrm{GFI}=.90\right.$, $\mathrm{AGFI}=.86, \mathrm{RMSEA}=.05$ with $90 \% \mathrm{CI}[.02-.07], \mathrm{CFI}=.87, \mathrm{NFI}=.64, \mathrm{ECVI}=1.51$, $\mathrm{AIC}=180.62]$

\section{Table 7}

Factorial Structure Matrix Rotated to the Varimax Criterion for PASES-C $(N=133)$

\begin{tabular}{|c|c|c|c|c|}
\hline Item Number & Barriers & $\begin{array}{l}\text { Support } \\
\text { Seeking }\end{array}$ & $\begin{array}{c}\text { Positive } \\
\text { Alternatives }\end{array}$ & $\begin{array}{c}\text { Communality } \\
\qquad\left(h^{2}\right)\end{array}$ \\
\hline PASES-C-2 & .12 & .78 & .15 & .64 \\
\hline PASES-C-3 & -.10 & -.02 & .82 & .69 \\
\hline PASES-C-5 & .09 & .58 & -.10 & .36 \\
\hline PASES-C-6 & .47 & .12 & .22 & .29 \\
\hline PASES-C-7 & -.06 & .48 & .39 & .38 \\
\hline PASES-C-8 & .39 & .26 & .01 & .22 \\
\hline PASES-C-9 & .01 & .69 & .18 & .51 \\
\hline PASES-C-10 & .62 & .05 & -.07 & .39 \\
\hline PASES-C-11 & .40 & .38 & -.05 & .30 \\
\hline PASES-C-12 & .14 & .25 & .47 & .30 \\
\hline PASES-C-13 & .73 & .11 & .06 & .54 \\
\hline PASES-C-14 & .62 & .07 & .17 & .42 \\
\hline PASES-C-15 & .39 & -.08 & .61 & .53 \\
\hline PASES-C-16 & .61 & -.08 & .05 & .38 \\
\hline
\end{tabular}




\begin{tabular}{lcccc}
\hline PASES-C-17 & .32 & .35 & -.06 & .23 \\
Eigenvalues & & & & \\
\% of variance explained & 21.19 & 1.64 & 1.33 & 6.16 \\
\hline
\end{tabular}

Factorial structure of the physical activity enjoyment scale. A CFA model was conducted to test the factorial structure of the PACES. Results of the CFA model indicated that the one-factor structure of the PACES did not fit the data well $\left[x^{2}(104)=\right.$ $262.81, p<.0001, \mathrm{CN}=62, \mathrm{SRMSR}=.10, \mathrm{GFI}=.76, \mathrm{AGFI}=.68, \mathrm{RMSEA}=.11$ with $90 \% \mathrm{CI}[.09-.13], \mathrm{CFI}=.70, \mathrm{NFI}=.60, \mathrm{ECVI}=2.67, \mathrm{AIC}=326.81]$. Thus, exploratory factor analysis with Varimax rotation was conducted to assess the factorial structure of the PACES. The results of the Bartlett's test indicated that there were significant correlations among the 16 items $\left(x^{2}=625.82, d f=120, p<.0001\right)$, and the KMO measures of sampling adequacy was .81, indicating that the factor analysis can yield distinct and reliable factors. A principle component analysis with Varimax rotation suggested a two-factor structure with all positively worded items loaded onto one factor, and all negatively worded items loaded onto the other factor except Item 7 , which loaded more on the positive component (See Table 8). For Item 7, Moore and colleges modified the word 'depressed' to 'sad' in 2009 from 2001 Motl's scale. The CFA model indicated that the two-factor structure fit the data better than one-factor structure, but still not adequately $\left[x^{2}(103)=173.19, p<.0001, \mathrm{CN}=93, \mathrm{SRMSR}=.08, \mathrm{GFI}=.86, \mathrm{AGFI}=.82\right.$, RMSEA $=.07$ with $90 \%$ CI [.05-.09], $\mathrm{CFI}=.87, \mathrm{NFI}=.74, \mathrm{ECVI}=1.72, \mathrm{AIC}=239.19]$. 


\section{Table 8}

Factorial Structure Matrix Rotated to the Varimax Criterion for PACES $(N=133)$

\begin{tabular}{|c|c|c|c|}
\hline Item Number & $\begin{array}{c}\text { Positively Worded } \\
\text { Items }\end{array}$ & $\begin{array}{c}\text { Negatively Worded } \\
\text { Items }\end{array}$ & $\begin{array}{l}\text { Communality } \\
\qquad\left(h^{2}\right)\end{array}$ \\
\hline PACES-1 & .51 & .07 & .26 \\
\hline PACES-2 & .02 & .66 & .44 \\
\hline PACES-3 & .28 & .72 & .59 \\
\hline PACES-4 & .35 & .12 & .13 \\
\hline PACES-5 & .07 & .67 & .46 \\
\hline PACES-6 & .66 & .05 & .43 \\
\hline PACES-7 & .46 & .39 & .36 \\
\hline PACES-8 & .47 & .39 & .38 \\
\hline PACES-9 & .85 & .03 & .72 \\
\hline PACES-10 & .42 & -.03 & .16 \\
\hline PACES-11 & .69 & .32 & .58 \\
\hline PACES-12 & .05 & .70 & .50 \\
\hline PACES-13 & .07 & .61 & .38 \\
\hline PACES-14 & .65 & .23 & .48 \\
\hline PACES-15 & .75 & .10 & .57 \\
\hline PACES-16 & .20 & .67 & .48 \\
\hline Eigenvalues & 4.89 & 2.05 & 6.94 \\
\hline$\%$ of variance explained & 30.57 & 12.78 & 43.35 \\
\hline
\end{tabular}

Factorial structure of the parental influence scale. A CFA model was

conducted to test the factorial structure of the PAIS. Results of the CFA model indicated that the two-factor structure of PAIS did not fit the data well $\left[x^{2}(134)=371.82, p<.0001\right.$, $\mathrm{CN}=55, \mathrm{SRMSR}=.11, \mathrm{GFI}=.74, \mathrm{AGFI}=.67, \mathrm{RMSEA}=.12$ with $90 \% \mathrm{CI}[.10-.13]$, 
$\mathrm{CFI}=.62, \mathrm{NFI}=.52, \mathrm{ECVI}=3.64, \mathrm{AIC}=445.82]$. Thus, exploratory factor analysis with Varimax rotation was conducted to assess the factorial structure of the PAIS. The results of Bartlett's test indicated that there were significant correlations among the 18 items $\left(x^{2}=734.80, d f=153, p<.0001\right)$, and the KMO measures of sampling adequacy was .76, indicating that the factor analysis can yield distinct and reliable factors. A principle component analysis with Varimax rotation suggested a two-factor structure with all negatively worded items loaded onto one factor, and all positively worded items loaded onto the other factor except Item 8, which loaded more on the negative component (See Table 9). The CFA model showed that the new two-factor structure fit the data better than the previous two-factor structure, though still not adequately $\left[x^{2}(134)=\right.$ $324.23, p<.0001, \mathrm{CN}=63, \mathrm{SRMSR}=.09, \mathrm{GFI}=.78, \mathrm{AGFI}=.72, \mathrm{RMSEA}=.11$ with $90 \% \mathrm{CI}[.09-.12], \mathrm{CFI}=.70, \mathrm{NFI}=.58, \mathrm{ECVI}=3.26, \mathrm{AIC}=398.23]$

\section{Table 9}

Factorial Structure Matrix rotated to the Varimax Criterion for PAIS $(N=133)$

\begin{tabular}{|c|c|c|c|}
\hline Item Number & $\begin{array}{c}\text { Positively Worded } \\
\text { Items }\end{array}$ & $\begin{array}{c}\text { Negatively Worded } \\
\text { Items }\end{array}$ & $\begin{array}{l}\text { Communality } \\
\qquad\left(h^{2}\right)\end{array}$ \\
\hline PAIS-1 & .67 & -.07 & .45 \\
\hline PAIS-2 & .38 & .23 & .20 \\
\hline PAIS-3 & .58 & -.10 & .42 \\
\hline PAIS-4 & .51 & .28 & .33 \\
\hline PAIS-5 & -.03 & .59 & .35 \\
\hline PAIS-6 & .60 & .19 & .40 \\
\hline PAIS-7 & -.03 & .72 & .52 \\
\hline PAIS-8 & .41 & .53 & .45 \\
\hline PAIS-9 & .04 & .66 & .44 \\
\hline PAIS-10 & .67 & .31 & .54 \\
\hline
\end{tabular}




\begin{tabular}{lccc}
\hline PAIS-11 & $\mathbf{. 7 0}$ & .11 & .51 \\
PAIS-12 & $\mathbf{. 5 0}$ & .25 & .31 \\
PAIS-13 & .09 & .77 & .60 \\
PAIS-14 & $\mathbf{. 3 0}$ & -.13 & .11 \\
PAIS-15 & $\mathbf{. 5 9}$ & -.10 & .36 \\
PAIS-16 & $\mathbf{. 6 1}$ & .03 & .38 \\
PAIS-17 & $\mathbf{. 4 6}$ & -.22 & .26 \\
PAIS-18 & $\mathbf{. 4 3}$ & .40 & .34 \\
& & & \\
Eigenvalues & 4.80 & 2.17 & 6.97 \\
\% of variance explained & 26.66 & 12.05 & 38.71 \\
\hline
\end{tabular}

Factorial structure of the modified environment scale. The results of Bartlett's test indicated that there were significant correlations among the 5 items $\left(x^{2}=72.59, d f=\right.$ $10, p<.0001)$, and the KMO measures of sampling adequacy was .62 , indicating that the factor analysis can yield distinct and reliable factors (Kaiser, 1974). A principal component analysis with a Varimax rotation was conducted, yielding a two-factor structure: equipment accessibility (items 1,2 and 5) and neighborhood safety (items 3 and 4 ). The two factors accounted for $36.18 \%$ and $26.72 \%$ of the variance respectively, with a total of $62.9 \%$ variance. The rotated factor structure coefficients and communality coefficients are provided in Table 10. A CFA was conducted to verify the two-factor structure of the modified environment scale. The CFA model showed that the two-factor structure fit the data adequately $\left[x^{2}(4)=1.31, p=.859, \mathrm{CN}=910, \mathrm{SRMSR}=.02, \mathrm{GFI}=\right.$ $1.00, \mathrm{AGFI}=.98, \mathrm{RMSEA}=.00$ with $90 \% \mathrm{CI}[.00-.07], \mathrm{CFI}=1.00, \mathrm{NFI}=.98, \mathrm{ECVI}$ $=.19, \mathrm{AIC}=23.31]$. 


\section{Table 10}

Environment Scale Factorial Structure Matrix Rotated to the Varimax Criterion $(N=133)$

\begin{tabular}{cccc}
\hline Item & $\begin{array}{c}\text { Equipment } \\
\text { Accessibility }\end{array}$ & $\begin{array}{c}\text { Neighborhood } \\
\text { Safety }\end{array}$ & $\begin{array}{c}\text { Communality } \\
\left(h^{2}\right)\end{array}$ \\
\hline Item 1 & $\mathbf{. 8 3}$ & .10 & .70 \\
Item 2 & $\mathbf{. 7 4}$ & -.02 & .55 \\
Item 3 & .13 &. $\mathbf{7 6}$ & .59 \\
Item 4 & -.07 & $\mathbf{. 8 6}$ & .75 \\
Item 5 & $\mathbf{. 7 2}$ & .03 & .52 \\
& & & \\
Eigenvalues & 1.81 & 1.34 & 3.15 \\
\% of variance explained & 36.18 & 27.72 & 62.9 \\
\hline
\end{tabular}

\section{Compliance Rate, Reliability, and Reactivity of Wearing Pedometers}

\section{Compliance Rate}

Among the 133 children, $87.2 \%(n=116)$ returned their pedometers, and $12.8 \%$ $(n=17)$ indicated they lost their pedometers. On average, $38.4 \%(n=51)$ of the children wore pedometers for seven days, $12 \%(n=16)$ wore pedometers for six days, $9 \%(n=12)$ wore pedometers for five days, and 3\% $(n=4)$ wore pedometers for four days, with a total of $62.4 \%(n=83)$ wore pedometers for more than three days. Only $5.3 \%(n=7)$ of the children did not wear pedometers, and 9\% $(n=12)$ wore pedometers for one day, $16.5 \%(n=22)$ wore pedometers for two days, and $6.8 \%(n=9)$ wore pedometers for three days.

\section{Reliability}

The ICCs were calculated using a two-way mixed-effect model with type consistency to estimate the reliability of daily pedometer steps. Starting with two 
consecutive days (Day 1 and Day 2), reliability of successive combination of days was estimated by adding one day at a time. The ICCs with $95 \%$ CI for daily pedometer steps are presented in Table 11. As expected, the reliability coefficients increased with the number of days of data increasing. All the reliability coefficients were strong even with only two days' data.

\section{Table 11}

Pedometer Reliability over Seven Consecutive Days $(N=133)$

\begin{tabular}{lccc}
\hline & & \multicolumn{2}{c}{$95 \%$ CI } \\
\cline { 3 - 4 } Days & ICC & Lower Bound & Upper Bound \\
\hline Day 1-2 & .70 & .54 & .80 \\
Day 1-3 & .79 & .70 & .86 \\
Day 1-4 & .85 & .78 & .90 \\
Day 1-5 & .85 & .78 & .90 \\
Day 1-6 & .86 & .79 & .91 \\
Day 1-7 & .87 & .81 & .92 \\
Weekdays & .80 & .70 & .87 \\
Weekend Days & .79 & .66 & .87 \\
\hline
\end{tabular}

\section{Reactivity}

Mixed linear regression model (Brown \& Prescott, 2006) was used to examine the change trend of children's pedometer steps over time, while paired-samples t-test was conducted to compare the differences of pedometer steps between two consecutive days. Table 12 demonstrates the mean and standard deviation of the pedometer steps over time. The results of the mixed linear regression model indicated that the pedometer steps did not change over time from Day 1 to Day $7[t(485)=-1.33, p=.1841]$, either change from 
Monday through Sunday $[t(485)=.80, p=.4258]$. Consistently, no significant differences were found between two consecutive days.

\section{Table 12}

Mean and Standard Deviation of Pedometer Steps over Time $(N=133)$

\begin{tabular}{lccccccc}
\hline Variable & \multicolumn{7}{c}{ Day 1 - Day 7 } \\
& Day 1 & Day 2 & Day 3 & Day 4 & Day 5 & Day 6 & Day 7 \\
\hline 00Steps & 8303 & 8329 & 8229 & 7958 & 7424 & 8051 & 7944 \\
& $(4227)$ & $(4329)$ & $(4902)$ & $(4620)$ & $(4244)$ & $(4538)$ & $(4919)$ \\
& \multicolumn{7}{c}{ Monday - Sunday } \\
Steps & 7859 & 7668 & 7924 & 8348 & 8380 & 8120 & 8072 \\
& Monday & Tuesday & Wednesday & Thursday & Friday & Saturday & Sunday \\
& $(4531)$ & $(4786)$ & $(3786)$ & $(4495)$ & $(4751)$ & $(4969)$ & $(4477)$ \\
\hline
\end{tabular}

\section{Physical and Sedentary Activity}

During the school year, 23 (17.3\%) children walked to school, only $2(1.5 \%)$ went to school by bicycle, 42 (31.6\%) children went to school by school bus, and $66(49.6 \%)$ went to school by car for most of the days. The screen-viewing time was not significantly correlated with self-reported physical activity levels $(r=-.091, p=.307)$, or pedometer steps $(r=.034, p=.708)$.

\section{Sedentary Activity}

About 104 (78.2\%) children watched TV/DVDs, 83 (62.4\%) played video games, and $110(82.7 \%)$ used computers during the school year, for an average of 57.31 minutes $(S D=52.82)$ per day. Analysis of the data reported by parents found that the majority of the children $(n=120,90.2 \%)$ met the screen-viewing time recommendation by AAP: viewing TV, videos or playing video games for no more than 2 hours a day. The length of 
screen-viewing time was significantly and negatively correlated with parental influence $(r$ $=-.23, p=.012)$ as well as the two components of parental influence: parental support $(r$ $=-.18, p=.043)$ and role modeling $(r=-.23, p=.01)$. Children using computers had more screen-viewing time than children who did not use computers [431 \pm 385 vs. $261 \pm$ $249, t(128)=-2.03, p=.044]$. Similarly, children watching TV/DVDs [448 \pm 383 vs. 239 $\pm 265, t(128)=-2.74, p=.007$ ], or playing video games [453 \pm 414 vs. $316 \pm 264, t(128)$ $=-2.30, p=.023]$ had more screen-viewing time. No statistically significant results were found related to the influence of other demographics on sedentary activity levels.

\section{Physical Activity}

Children took an average of 7,868 $(S D=3526)$ pedometer steps per day. Only one 8-year-old girl had at least 11,000 steps each day. When using the average pedometer steps to assess children's status of meeting physical activity recommendations, $13.5 \%$ ( $n$ $=10)$ of boys and $11.9 \%(n=7)$ of girls, total $12.8 \%(n=17)$ of the children, met physical activity recommendations, that is, 13,000 steps/day for boys and 11,000 steps/day for girls. Cut points for boys are: (1) < 10,000 (sedentary), (2) 10,000-12,499 (low active), (3) 12,500-14,999 (somewhat active), (4) 15,000 - 17,499 (active), and (5) $\geq$ 17,500 (highly active) steps/day; and for girls are: (1) <7,000, (2) 7,000-9,499, (3) 9,50011,999, (4) 12,000 - 14,499, and (5) $\geq 14,500$ steps/day (Tudor-Locke et al., 2011). Based on these cut points, approximately $59.5 \%(n=44)$ boys and $52.5 \%(n=31)$ girls were sedentary; $18.9 \%(n=14)$ boys and $30.5 \%(n=18)$ girls were low active; $9.5 \%(n=7)$ boys and 6.8\% $(n=4)$ girls were somewhat active; $4.1 \%(n=3)$ boys and $5.1 \%(n=3)$ girls were active; and only $4 \%(n=2)$ girls were highly active. 
On average, boys took more pedometer steps than girls $[8441 \pm 3685$ vs. $7197 \pm$ $3233, t(124)=2.02, p=.046]$. Children with a full-time employed mother tended to have fewer pedometer steps than children with an unemployed or part-time employed mother $[7429 \pm 3306$ vs. $8717 \pm 3815, t(124)=1.97, p=.052]$. Mode of transportation to school had a significant influence on children's pedometer steps $[F(3,122)=4.16, p=.008]$. The results of Hochberg's GT2 post-hoc tests indicated that children who walked to school $(10083 \pm 3784)$ had the highest pedometer steps compared to children who went to school by school bus $(7561 \pm 3634, p=.037)$, or by car $(7380 \pm 3126, p=.01)$, but not by bicycle ( $4895 \pm 1180, p=.22)$ however only two children went to school by bicycle.

Children from families with annual family income less than $\$ 30,000$ reported higher self-reported physical activity levels than children from families with annual family income greater than $\$ 30,000$, but not statistically significant (3.34 \pm .67 vs. 3.09 $\pm .73, p=.059)$. Children having a full-time employed father reported participating in lower physical activity levels than children with an unemployed or part-time employed father $(3.08 \pm .74$ vs. $3.37 \pm .65, p=.046)$. Similarly, children having a full-time employed mother tended to have lower self-reported physical activity levels children with an unemployed or part-time employed mother, though not statistically significant (3.12 \pm .72 vs. $3.36 \pm .72, p=.067)$. In addition, children with a mother who received at least some college education tended to have higher self-reported physical activity levels than children with a mother who did not receive any college education $(3.30 \pm .72$ vs. 3.06 $\pm .66, p=.061)$. 


\section{Obesity Status}

For children participating in this study, 2.3\% $(n=3)$ were underweight and $48.9 \%$ ( $n=65)$ were healthy weight, while $18.8 \%(n=25)$ were overweight and $30.1 \%(n=40)$ were obese. The average BMI was $20.74(S D=5.12$, range: $13.40-38.20)$, the average BMI-percentile was 73.97 ( $S D=26.45$, range: 3.20-99.60), and the average BMI z-score was $.94(S D=1.07$, range: $-1.84-2.74)$. Hispanic children tended to have higher BMIpercentile $(81.50 \pm 21.16$ vs. $72.05 \pm 27.39, p=.097)$, and BMI z-score $(1.28 \pm .98$ vs. $.85 \pm 1.08, p=.065)$ than Non-Hispanic children, but not statistically significant. Children with a mother who did not receive any college education had higher BMIpercentile $(80.28 \pm 20.55$ vs. $69.23 \pm 29.37, p=.012)$, and BMI z-score $(1.17 \pm .90$ vs. $.76 \pm 1.17, p=.028)$ than children with a mother who received at least some college education.

Overweight or obese children had lower scores on barriers component of the PASES than non-overweight children $(2.61 \pm 1.39$ vs. $3.12 \pm 1.05, p=.021)$. Children's BMI-percentile was significantly and negatively correlated with physical activity selfefficacy $(r=-.19, p=.038)$, specifically the barriers component $(r=-.20, p=.024)$. Children's BMI z-score was also significantly and negatively correlated with physical activity self-efficacy $(r=-.21, p=.02)$, specifically the support seeking component $(r=-$ $.18, p=.045)$ and barriers component $(r=-.21, p=.018)$. Children's BMI $(r=-.27, p$ $=.002)$ and BMI z-score $(r=-.18, p=.042)$, but not BMI-percentile $(r=-.10, p=.252)$, was negatively correlated with pedometer steps. Overweight or obese children had fewer pedometer steps $[7022 \pm 3037$ vs. $8741 \pm 3798, t(124)=2.81, p=.006]$, and fewer days 
of meeting physical activity recommendations $[.48 \pm .95$ vs. $.94 \pm 1.65, t(108)=2.0, p$ $=.048]$ than non-overweight children.

A binary logistic regression model was conducted to model the relationship between 14 predictors of children's obesity status $(1=$ overweight/obese, $0=$ nonoverweight). The demographic factors (age, sex, ethnicity, race, number of children, marital status, family income, employment status, and education levels), self-reported physical activity levels, pedometer steps, and screen-viewing time were entered into the logistic regression model. All the categorical demographic variables were coded as a dummy variable. Results indicated a statistically significant improvement over the constant-only model $\left[x^{2}(17, N=97)=34.14, p=.008\right]$. Only father's employment status $(1=$ full time, $0=$ part time or unemployed $)(p=.002)$, mother's education level $(1=$ some college education, $0=$ no college education $)(p=.034)$, ethnicity $(1=$ Hispanic, $0=$ Non-Hispanic) $(p=.026)$, children's age $(p=.004)$, and self-reported physical activity levels $(p=.021)$ were the significant predictors of children's obesity status. The model predicted that the odds for a child with a part-time or unemployed father to be overweight or obese were 12 times higher than a child with a full-time employed father, $O R=12.35$, $95 \% C I=[2.49-62.5]$. Moreover, the odds for a child with a mother who did not receive any college education to be overweight or obese were about 5 times than a child with a mother who received some college education, $O R=4.83,95 \% C I=[1.12-20.76]$. Hispanic children were 9 times more likely to be overweight or obese than Non-Hispanic children, $O R=8.93,95 \% C I=[1.30-62.5]$. With self-reported physical activity levels, $O R=2.65,95 \% C I=[1.16-6.09]$, and children's age, $O R=2.43,95 \% C I=[1.32-4.46]$, increased by one point, the odds for children to be overweight or obese were doubled. 


\section{Determinants of Physical Activity}

The mean and standard deviation for each determinant are demonstrated in Table

6. Girls had higher scores on physical activity self-efficacy (14.15 \pm 2.54 vs. $12.84 \pm 3.15$, $p=.01)$ : support seeking component $(6.34 \pm .99$ vs. $5.71 \pm 1.51, p=.006)$, and positive alternatives component ( $4.85 \pm 1.19$ vs. $4.34 \pm 1.35, p=.026)$; and equipment accessibility component $(12.47 \pm 2.21$ vs. $11.22 \pm 3.08, p=.009)$. Race had significant effect on children's perceived parental influence $[F(4,114)=3.16, p=.017]$, specifically parental support component $[F(4,114)=3.22, p=.015)$. Results of the Hochberg's GT2 post-hoc tests indicated that compared to African American children, mixed-race children perceived to receive more parental influence $(49.92 \pm 8.48$ vs. $58.27 \pm 7.25, p=.036)$, and parental support $(33.84 \pm 6.50$ vs. $40.00 \pm 5.33, p=.037)$. As children aged, their barriers component of the PASES decreased $(r=-.18, p=.039)$. Children from a family with an annual family income less than $\$ 20,000$ had the highest physical activity enjoyment level $[F(3,117)=2.93, p=.036]$.

Mother's employment status had significant influence on children's perceptions of parental influence $[F(2,124)=3.58, p=.031]$, and role modeling component $[F(2,124)$ $=4.97, p=.008]$. Specifically, children with an unemployed mother had higher scores on parental influence $(57.39 \pm 8.15$ vs. $51.49 \pm 8.80, p=.019)$, and role modeling component than children with a full-time employed mother $(18.50 \pm 4.76$ vs. $16.03 \pm$ $3.43, p=.018)$. Children with an unemployed or part-time employed mother had higher scores on positive alternatives component of the PASES compared to children with a fulltime employed mother $(4.93 \pm 1.18$ vs. $4.40 \pm 1.32, p=.03)$. Children with a father who received some college education had higher scores on positive alternatives component of 
the PASES than children with a father who did not receive any college education $(4.92 \pm$ 1.21 vs. $4.37 \pm 1.35, p=.025)$. Children with a mother who received some college education had higher physical activity enjoyment than children with a mother who did not receive any college education $(68.41 \pm 8.39$ vs. $64.72 \pm 10.08, p=.027)$.

\section{Research Questions}

\section{Research Question One}

The first research question asked what the influence of wearing pedometers on the determinants (physical activity self-efficacy, physical activity enjoyment, parental influence, and environment) of physical activity was. Paired-samples t-test was used to examine the influence of wearing pedometers on the hypothesized determinants of physical activity among elementary school children attending afterschool programs. No significant differences were found on the determinants of physical activity after one-week pedometer wearing (See Table 13).

\section{Table 13}

Effects of Wearing Pedometers on the Determinants of Physical Activity $(n=50)$

\begin{tabular}{lcccc}
\hline Variable & Pre-test & Post-test & $t$-statistic & $p$-value \\
& $M \pm S D$ & $M \pm S D$ & & \\
\hline Self-efficacy & $13.31 \pm 3.47$ & $13.43 \pm 3.32$ & -.33 & .744 \\
$\quad$ Support seeking & $5.82 \pm 1.48$ & $5.90 \pm 1.56$ & -.41 & .684 \\
$\quad$ Barriers & $3.18 \pm 1.13$ & $2.98 \pm 1.23$ & 1.70 & .096 \\
$\quad$ Positive alternatives & $4.31 \pm 1.57$ & $4.55 \pm 1.42$ & -1.27 & .209 \\
Enjoyment & $66.43 \pm 8.78$ & $67.16 \pm 9.33$ & -.55 & .587 \\
Parental Influence & $52.65 \pm 8.89$ & $52.55 \pm 9.96$ & .10 & .919 \\
$\quad$ Role modeling & $16.96 \pm 3.52$ & $16.84 \pm 4.16$ & .28 & .783 \\
$\quad$ Parental support & $35.69 \pm 6.55$ & $35.71 \pm 7.05$ & -.02 & .982 \\
Environment & $18.39 \pm 4.07$ & $18.39 \pm 3.99$ & 0 & 1.0 \\
\hline
\end{tabular}




\begin{tabular}{lcccc}
\hline Access & $11.92 \pm 2.32$ & $11.84 \pm 2.69$ & .20 & .841 \\
Safety & $6.47 \pm 2.69$ & $6.55 \pm 2.44$ & -.21 & .836 \\
\hline
\end{tabular}

\section{Research Question Two}

The second research question asked what the interrelationships among physical activity self-efficacy, physical activity enjoyment, parental influence, environment and physical activity were. Pearson correlation analysis was conducted to assess the interrelationships among the hypothesized determinants of physical activity: physical activity self-efficacy, physical activity enjoyment, parental influence, environment and physical activity (See Table 14). Self-reported physical activity levels were significantly and moderately correlated with physical activity self-efficacy, physical activity enjoyment, parental influence and environment. Pedometer steps were not significantly correlated with any hypothesized determinant, but were significantly correlated with role modeling component of the PAIS ( $r=.20, p=.031)$. All the hypothesized determinants of physical activity were significantly correlated with each other. As shown in Figure 5, only a non-significant weak correlation was found between self-reported physical activity levels and pedometer steps $(r=.14, p=.116)$. 


\section{Table 14}

Interrelationships among Variables $(N=133)$

\begin{tabular}{|c|c|c|c|c|c|}
\hline Variable & $\begin{array}{c}\text { Self- } \\
\text { reported PA }\end{array}$ & $\begin{array}{l}\text { Pedometer } \\
\text { Steps }\end{array}$ & $\begin{array}{c}\text { Self- } \\
\text { efficacy }\end{array}$ & Enjoyment & $\begin{array}{l}\text { Parental } \\
\text { Influence }\end{array}$ \\
\hline $\begin{array}{l}\text { Pedometer } \\
\text { Steps }\end{array}$ & .14 & & & & \\
\hline Self-efficacy & $.41 * *$ & .07 & & & \\
\hline Enjoyment & $.45^{* *}$ & -.001 & $.40 * *$ & & \\
\hline $\begin{array}{l}\text { Parental } \\
\text { Influence }\end{array}$ & $.43 * *$ & .17 & $.46^{* *}$ & $.37 * *$ & \\
\hline Environment & $.39 * *$ & .05 & $.37 * *$ & $.37 * *$ & $.59 * *$ \\
\hline
\end{tabular}

Note. ${ }^{* *} p<.01$

\section{Figure 5}

$\underline{\text { Scatterplot between Self-reported Physical Activity Levels and Pedometer Steps }}$

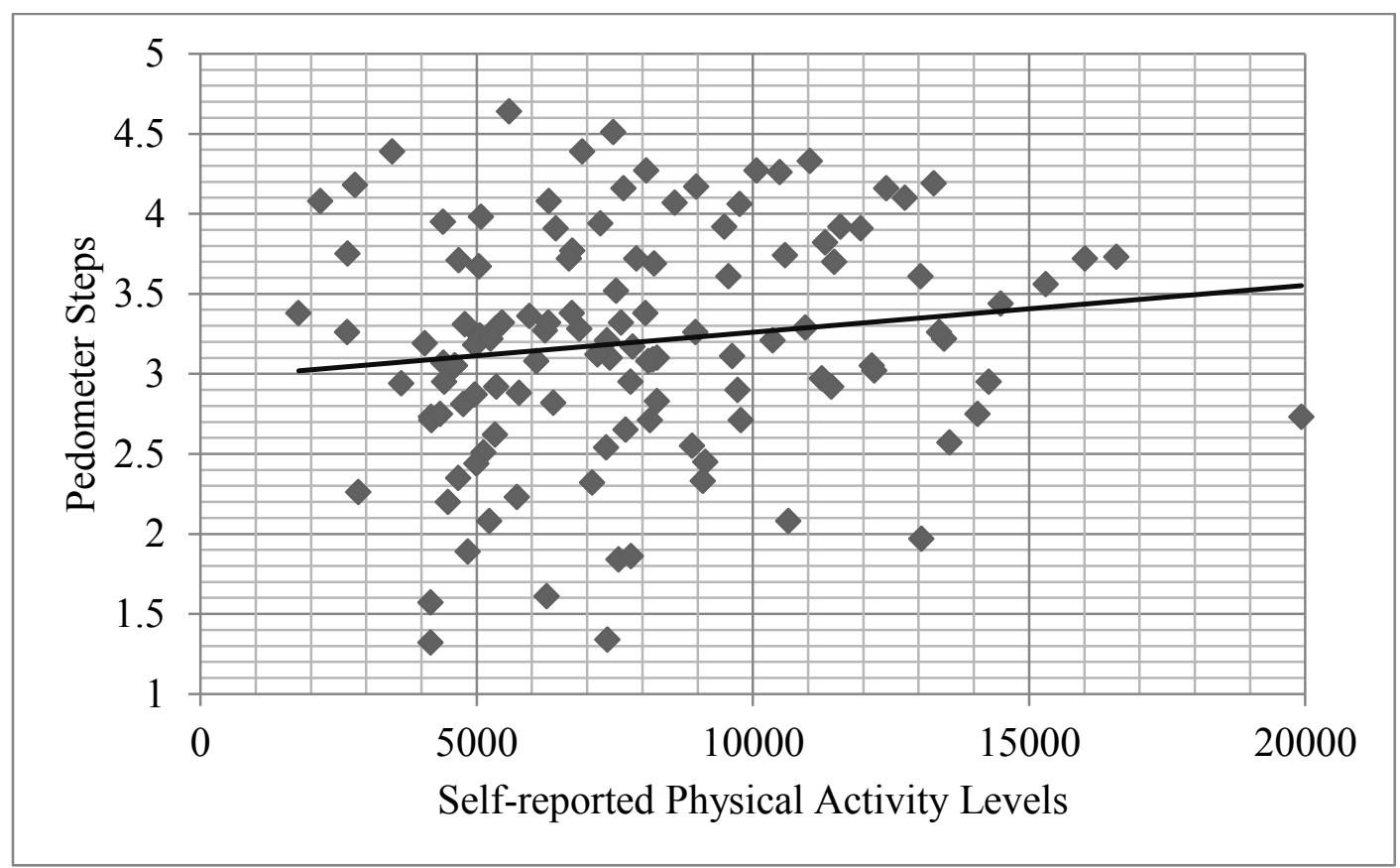

\section{Research Question Three}

The third research question asked what the significant psychosocial and environment determinants of self-reported physical activity levels were among children 
aged 8 to 11 years old. The SEM with maximum likelihood (ML) was used to examine the determinants of self-reported physical activity levels.

Parental influence. Model A (See Figure 6 Model A) demonstrates the best-fitted self-reported physical activity model based on the YPAP model after deleting the nonsignificant path between perceived environment and physical activity. In Model A, both physical activity self-efficacy $(\beta=.19, p=.02)$, and physical activity enjoyment $(\beta=.29$, $p=.0003$ ) had significant direct effect on self-reported physical activity levels.

Children's perceived parental influence had both direct $(\beta=.24, p=.007)$, and indirect effect $(\beta=.19, p=.0003)$ through physical activity self-efficacy and enjoyment, on selfreported physical activity levels. Perceived environment did not have any significant effect on self-reported physical activity levels. Children's perceived parental influence accounted for about $14 \%$ of the variance of physical activity enjoyment, and $21 \%$ of the variance of physical activity self-efficacy. This model could significantly explain $28 \%$ of the variance of self-reported physical activity levels. However, the model fit indices were less than adequate (See Table 15).

Model B was solely developed based on this study data (See Figure 6 Model B). In Model B, children's perceived parental influence served as the partial mediator of the relationships of physical activity self-efficacy and physical activity enjoyment with physical activity. Physical activity self-efficacy had significant direct effect $(\beta=.19, p$ $=.03)$, and indirect effect $(\beta=.09, p=.02)$ through perceived parental influence, on selfreported physical activity levels. Physical activity enjoyment had significant direct effect $(\beta=.28, p=.0005)$, but non-significant indirect effect $(\beta=.05, p=.05)$ through perceived parental influence, on self-reported physical activity levels. Perceived parental 
influence had significant direct effect $(\beta=.23, p=.005)$ on self-reported physical activity levels. Physical activity self-efficacy and physical activity enjoyment accounted for about $25 \%$ of the variance of perceived parental influence, and Model B could significantly explain about $30 \%$ of the variance of self-reported physical activity levels.

Compared to Model A, Model B fit the data adequately with smaller values of ECVI and AIC. In Model A, perceived parental influence had the strongest effect $(\beta=.43$, $p<.0001)$, and physical activity enjoyment had the second strongest effect $(\beta=.29, p$ $=.0003)$ on self-reported physical activity levels. While in Model B, physical activity enjoyment had the strongest effect $(\beta=.33, p<.0001)$, and physical activity self-efficacy had the second strongest effect $(\beta=.28, p=.0007)$ on self-reported physical activity levels. However, part of these effects (total $\beta=.14$ ) on self-reported physical activity levels in Model B were indirect effects mediated by perceived parental influence. Therefore, Model B is the best model for this study data, and perceived parental influence partially mediated the relationships of physical activity self-efficacy and physical activity enjoyment with self-reported physical activity.

Parental support. Considering the strong influence of perceived parental influence on physical activity, new SEM models were developed for the two subscales of parental influence, perceived parental support and perceived parental role modeling respectively. Model C (Figure 6) shows that perceived parental support had direct effect $(\beta=.24, p=.004)$, and indirect effect $(\beta=.18, p=.0002)$ through physical activity selfefficacy and physical activity enjoyment on self-reported physical activity levels. Physical activity self-efficacy $(\beta=.21, p=.0088)$, and physical activity enjoyment $(\beta$ $=.28, p=.0003$ ) had direct effect on self-reported physical activity levels. Perceived 
parental support could account for $12 \%$ variance of physical activity enjoyment and $14 \%$ variance of physical activity self-efficacy. The total model could explain about $29 \%$ variance of self-reported physical activity levels. However, based on the fit indices in Table 15, Model C did not fit the data well.

In Model D, perceived parental support was the partial mediator between physical activity self-efficacy, physical activity enjoyment, and physical activity. In this model, physical activity self-efficacy had direct effect $(\beta=.21, p=.0128)$, and indirect effect ( $\beta$ $=.07, p=.0268)$ through perceived parental support on self-reported physical activity levels. Similarly, physical activity enjoyment had direct effect $(\beta=.28, p=.0006)$, and indirect effect $(\beta=.06, p=.0474)$ through perceived parental support on physical activity. Perceived parental support had significantly direct effect on self-reported physical activity levels $(\beta=.24, p=.0031)$. Physical activity self-efficacy and physical activity enjoyment could explain about 19\% variance of perceived parental support, and this model accounted for about $31 \%$ variance of self-reported physical activity levels. Compared to Model C, Model D fit data better and adequately (See Table 15). Thus, Model D was selected as the final model for perceived parental support.

Parental role modeling. In Figure 6 Model E, physical activity self-efficacy ( $\beta$ $=.28, p=.0002)$, and physical activity enjoyment $(\beta=.34, p<.0001)$ had direct effect on self-reported physical activity levels, while perceived parental role modeling only had indirect effect $(\beta=.23, p<.0001)$ through physical activity self-efficacy and physical activity enjoyment on physical activity levels. In this model, perceived parental role modeling could account for $9 \%$ variance of physical activity enjoyment, and $20 \%$ 
variance of physical activity self-efficacy. This model could explain about $22 \%$ variance of self-reported physical activity levels.

In Figure 6 Model F, the same as Model E, physical activity self-efficacy $(\beta=.28$, $p<.001)$ and physical activity enjoyment $(\beta=.33, p<.001)$ had direct effect on selfreported physical activity levels, while perceived parental role modeling did not have any significant effect on physical activity levels. Although, the path between physical activity enjoyment and perceived parental role modeling was not significant, the investigator decided to keep this path after comparing the fit indices values between the two models. Physical activity self-efficacy and physical activity enjoyment together could explain about $22 \%$ variance of perceived parental role modeling, and $26 \%$ variance of selfreported physical activity levels. Compared to Model E, Model F fit this data better but not adequately (See Table 15). However, after comparing the two models related to the interrelationships among the study variables, Model E was selected as the final model for perceived parental role modeling.

Summary. After comparing fit indices values and path coefficients, Model B, Model D, and Model E were selected as the final models for Research Question Three. Perceived parental influence as well as perceived parental support partially mediated the relationships of physical activity self-efficacy and physical activity enjoyment with selfreported physical activity levels. Physical activity self-efficacy and physical activity enjoyment had direct and indirect effect through perceived parental influence or support on self-reported physical activity levels. Perceived parental role modeling only had indirect effect on self-reported physical activity levels through physical activity selfefficacy and physical activity enjoyment. 


\section{Figure 6}

$\underline{\text { Self-Reported Physical Activity Models }(N=133)}$

\section{Model A Parental Influence}

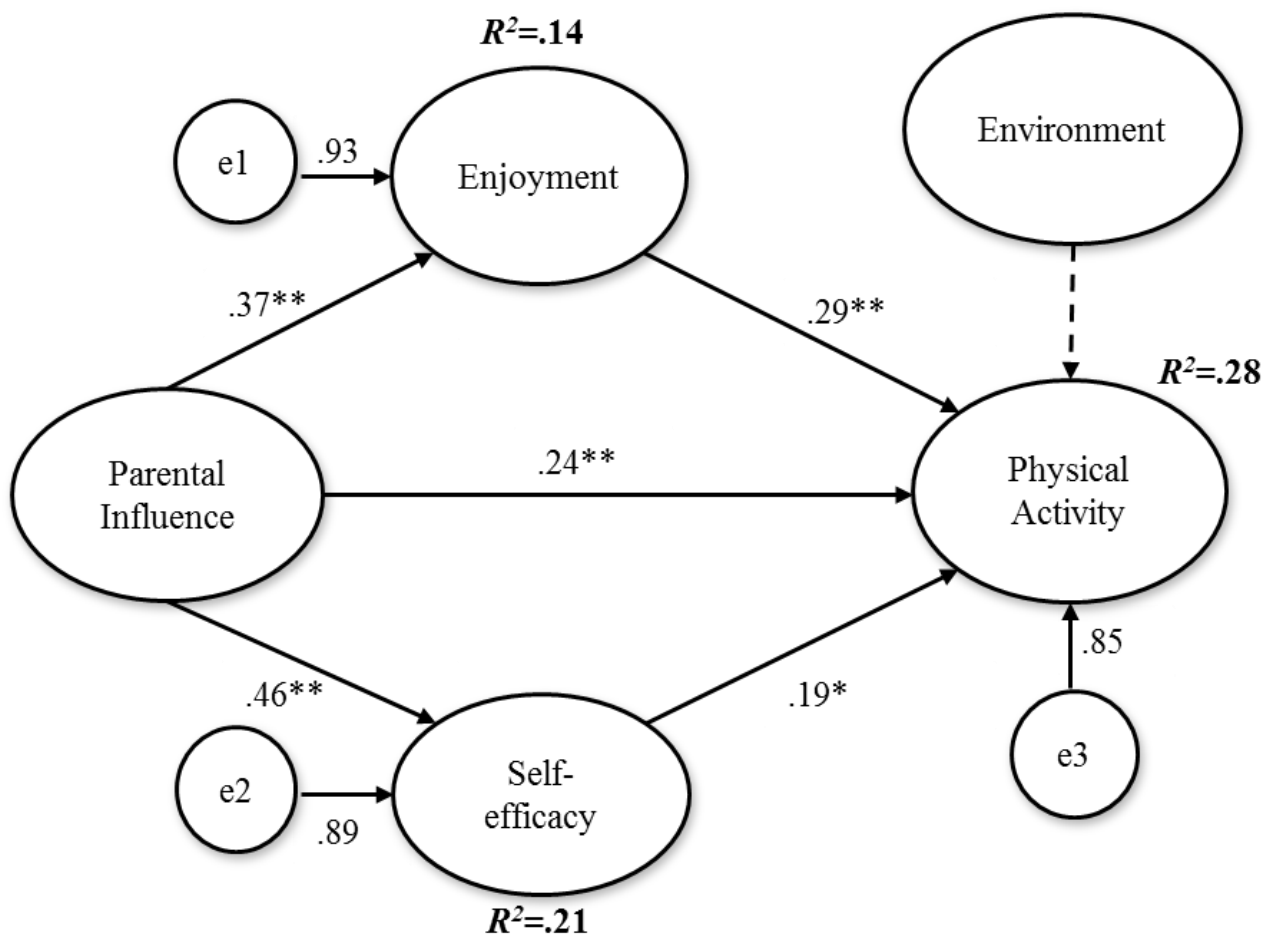

\section{Model B Parental Influence}

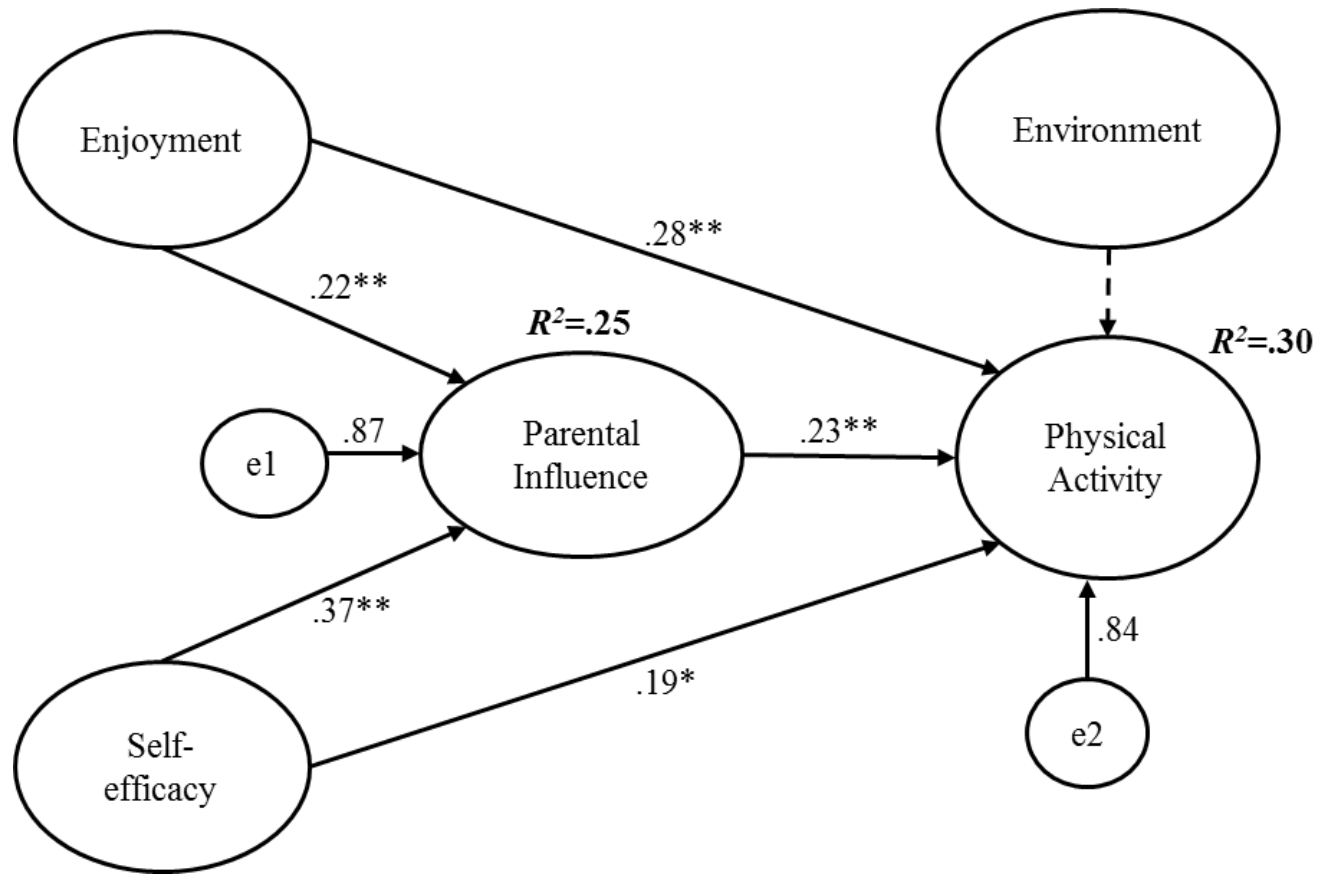




\section{Model C Parental Support}

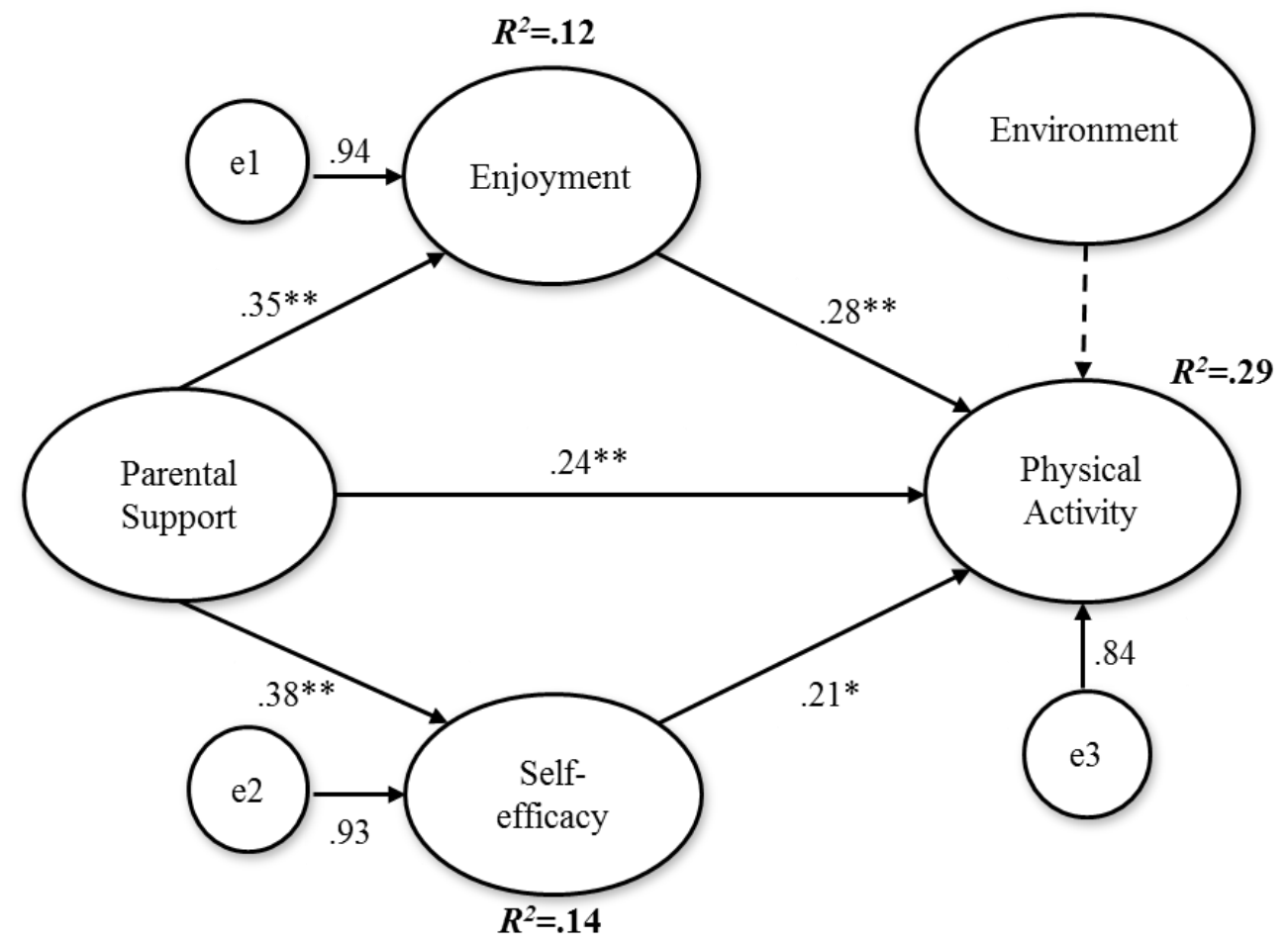

\section{Model D Parental Support}

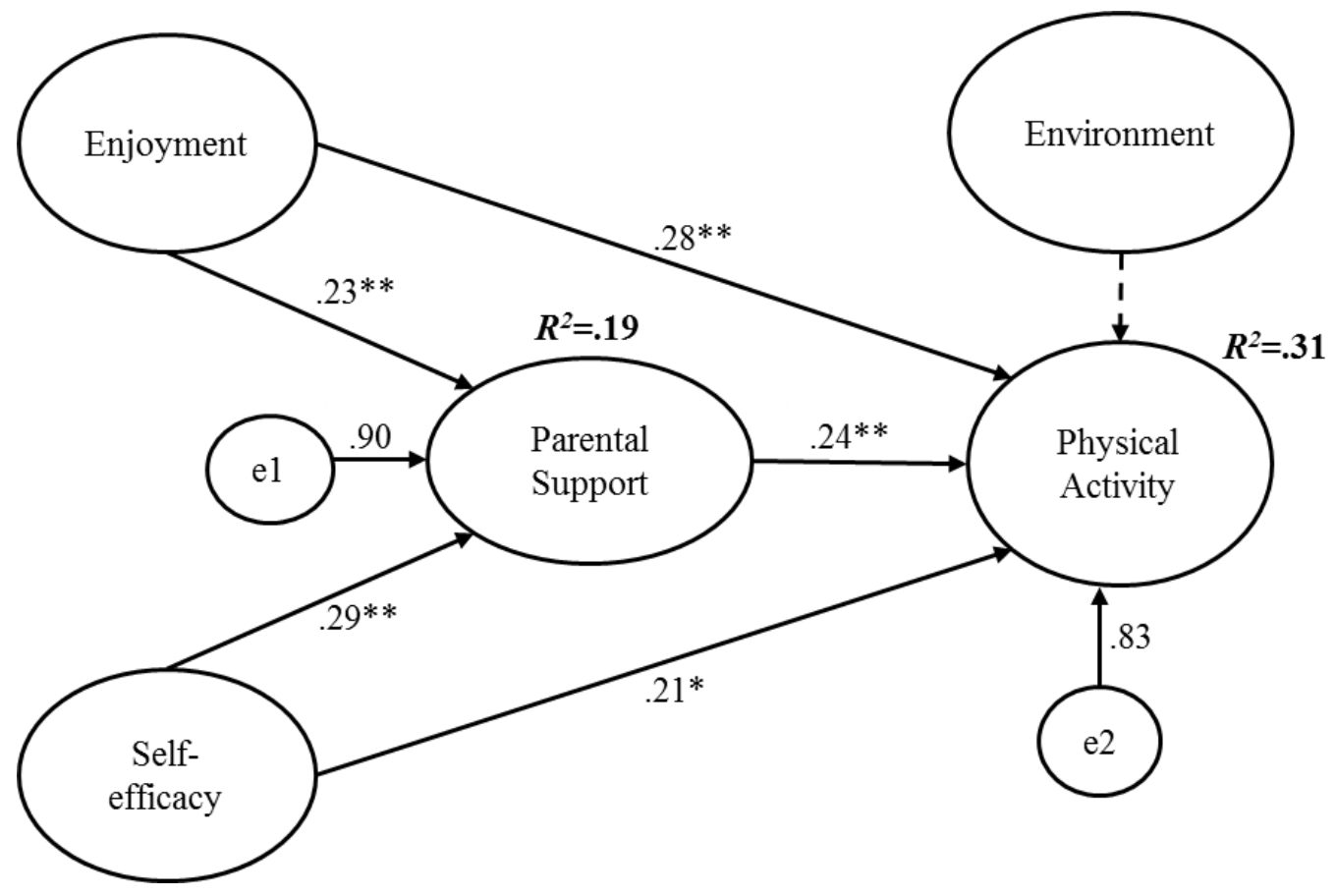




\section{Model E Parental Role Modeling}

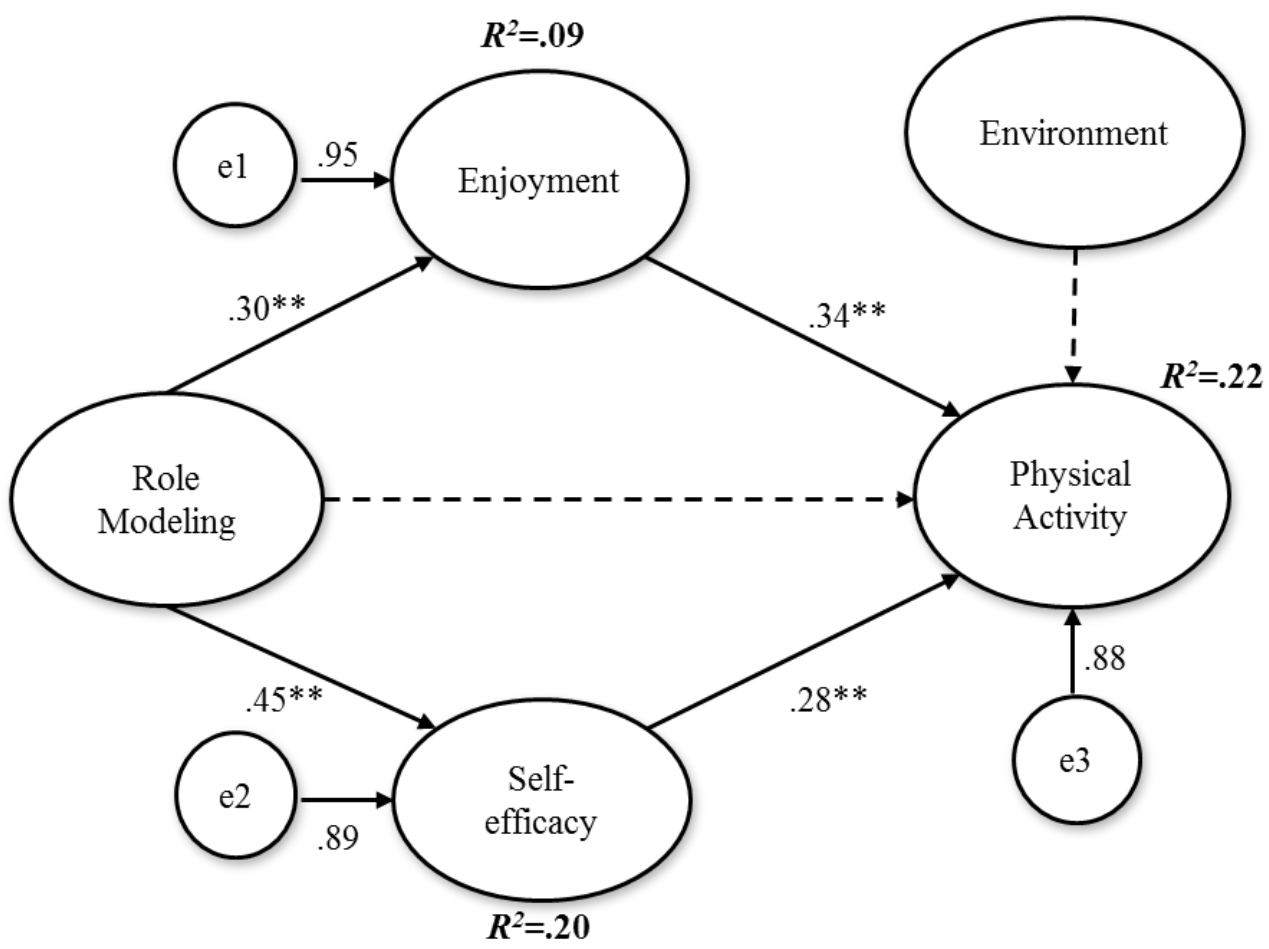

\section{Model F Parental Role Modeling}

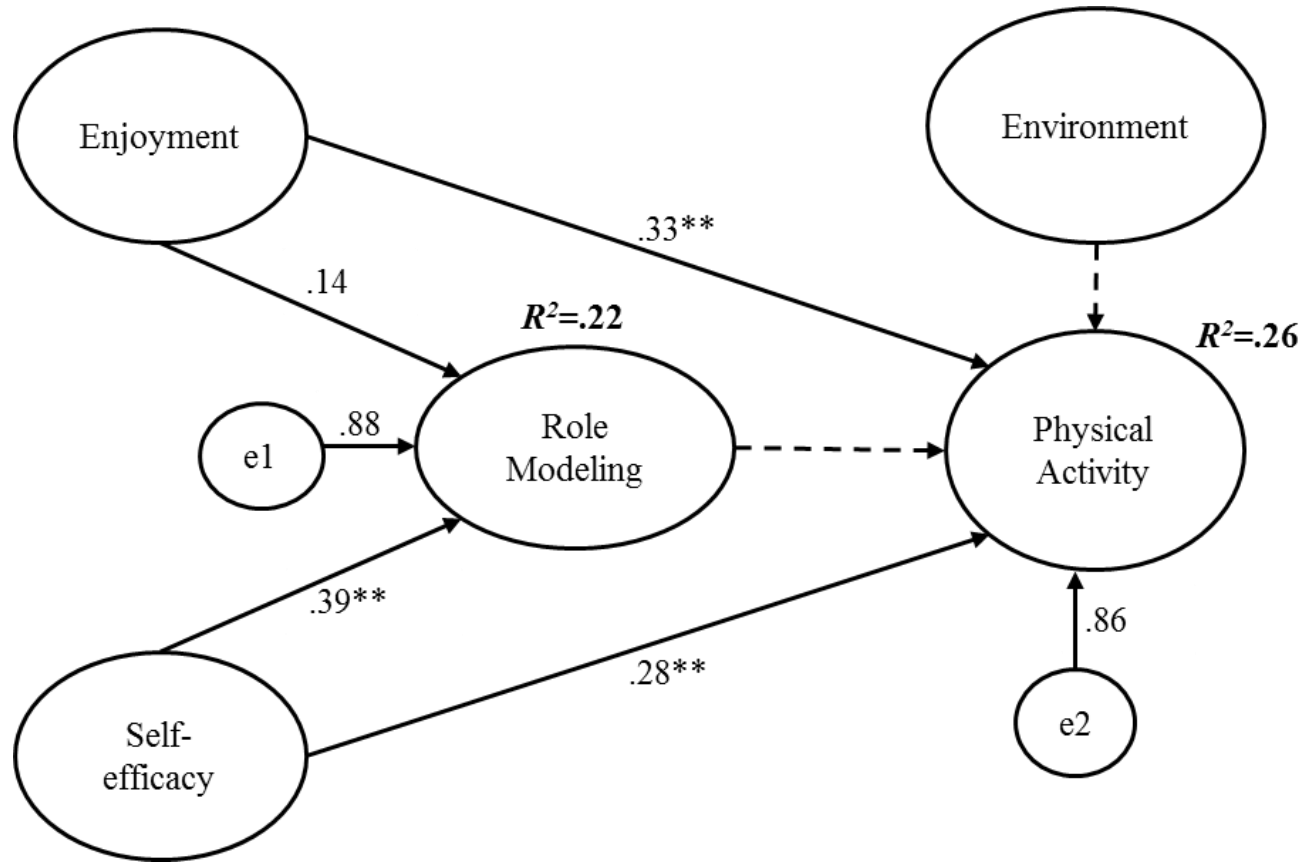

Note. ${ }^{*} p<.05,{ }^{* *} p<.01$. Model B, D, and E were selected as the final models for selfreported physical activity levels. 


\section{Table 15}

Model Fit Indices of Theoretical Model, Model A, Model B, Model C, Model D, Model E, and Model F $(N=133)$

\begin{tabular}{|c|c|c|c|c|c|c|c|c|}
\hline \multirow[b]{2}{*}{ Fit Index } & \multirow[b]{2}{*}{ Cut Point } & \multirow[b]{2}{*}{ Theoretical } & \multicolumn{2}{|c|}{ Parental Influence } & \multicolumn{2}{|c|}{ Parental Support } & \multicolumn{2}{|c|}{ Role Modeling } \\
\hline & & & Model A & Model B & Model C & Model D & Model E & Model F \\
\hline \multicolumn{9}{|l|}{ Absolute Fit } \\
\hline Chi-square test & $P>.05$ & .0008 & .0013 & $>.05$ & .0004 & $>.05$ & .0006 & .1549 \\
\hline Hoelter Critical N & $>200$ & 59 & 47 & $>200$ & 39 & $>200$ & 51 & 240 \\
\hline GFI & $>.90$ & .95 & .96 & 1.00 & .95 & 1.00 & .95 & .99 \\
\hline \multicolumn{9}{|l|}{ Parsimony Fit } \\
\hline AGFI & $>.95$ & .74 & .62 & 1.00 & .54 & 1.00 & .73 & .92 \\
\hline RMSEA & $<.06$ & $\begin{array}{c}.19 \\
(.11-.28)\end{array}$ & $\begin{array}{c}.27 \\
(.14-.43)\end{array}$ & 0 & $\begin{array}{c}.30 \\
(.17-.46)\end{array}$ & 0 & $\begin{array}{c}.23 \\
(.13-.34)\end{array}$ & $\begin{array}{c}.09 \\
(0-.27)\end{array}$ \\
\hline ECVI & Smaller & .33 & .23 & .17 & .25 & .17 & .25 & .16 \\
\hline AIC & Smaller & 40.70 & 28.35 & 20 & 30.71 & 20 & 30.89 & 20.02 \\
\hline \multicolumn{9}{|l|}{ Incremental Fit } \\
\hline Bentler CFI & $>.95$ & .91 & .90 & 1.00 & .87 & 1.00 & .85 & .99 \\
\hline
\end{tabular}

Note. Model B, D, and E were the best models. 


\section{Research Question Four}

The fourth research question asked what the significant psychosocial and environment determinants of objective physical activity levels measured by average pedometer steps were among children aged 8 to 11 years old. SEM with full information maximum likelihood (FIML) was conducted to examine the determinants of average pedometer steps.

Parental influence. Model A was developed based on the theoretical model. In this model (See Figure 7 model A), only perceived parental influence had significant direct effect on average pedometer steps $(\beta=.17, p=.0169)$. Perceived parental influence accounted for about 13\% variance of physical activity enjoyment, $21 \%$ variance of physical activity self-efficacy, and only $3 \%$ variance of average pedometer steps. The model fit indices suggested that this model fit data inadequately.

Model B was solely developed based on the data, and perceived parental influence served as the partial mediator. Based on the fit indices values, the nonsignificant path between physical activity self-efficacy and average pedometer steps was retained. In Model B (See Figure 7 Model B), perceived parental influence had significantly direct effect on average pedometer steps $(\beta=.18, p=.0143)$, while physical activity self-efficacy had significantly indirect effect through perceived parental influence on average pedometer steps $(\beta=.06, p=.0309)$. Physical activity enjoyment tended to have indirect effect on average pedometer steps through perceived parental influence, though not statistically significant $(\beta=.04, p=.0672$ ). Physical activity self-efficacy and physical activity enjoyment together could account for about $25 \%$ variance of perceived parental influence, and this model total could explain about $3 \%$ variance of average 
pedometer steps. Compared to Model A (See Table 16), Model B fit the data better. In Model $\mathrm{B}$, perceived parental influence had the strongest effect $(\beta=.17)$ on average pedometer steps, and mediated the relationships of physical activity self-efficacy and physical activity enjoyment with average pedometer steps.

Parental support. In Figure 7 Model C, only perceived parental support had significantly direct effect on average pedometer steps $(\beta=.13, p<.01)$. Perceived parental support could account for $12 \%$ variance of physical activity enjoyment, and $14 \%$ variance of physical activity self-efficacy. This model explained only $2 \%$ variance of average pedometer steps. In Figure 7 Model D, perceived parental support was the partial mediator. Both physical activity self-efficacy $(\beta=.03, p=.0021)$, and physical activity enjoyment $(\beta=.02, p=.0107)$ had significantly indirect effect through perceived parental support on average pedometer steps. Perceived parental support had significantly direct effect on average pedometer steps $(\beta=.11, p<.0001)$. Physical activity selfefficacy and physical activity enjoyment could explain about 19\% variance of perceived parental support, and Model D could account for only $1 \%$ variance of average pedometer steps. The fit indices values in Table 16 shows that Model D fit the data better compared to Model C. Therefore, Model D was selected as the final model for perceived parental support.

Parental role modeling. In Figure 7 Model E, only perceived parental role modeling had significant effect on average pedometer steps $(\beta=.19, p<.01)$. Perceived parental role modeling accounted for about $9 \%$ variance of physical activity enjoyment, and $20 \%$ variance of physical activity self-efficacy respectively. Model E could explain about 4\% variance of average pedometer steps. In Figure 7 Model F, perceived parental 
role modeling had direct effect on average pedometer steps $(\beta=.20, p<.0001)$, and physical activity self-efficacy had indirect effect through perceived parental role modeling on average pedometer steps $(\beta=.09, p<.0001)$. Physical activity self-efficacy accounted for about $20 \%$ variance of perceived parental role modeling, and this model explained about $4 \%$ variance of average pedometer steps. The fit indices values in Table 16 show that Model F fit data better and adequately. Thus, Model F was selected as the final model for perceived parental role modeling.

Summary. Model B, Model D, and Model F were selected as the final models for average pedometer steps. Perceived parental influence, parental support and parental role modeling had the strongest effect on average pedometer steps compared to physical activity self-efficacy and physical activity enjoyment. Perceived parental influence and parental support partially mediated the relationships of physical activity self-efficacy and physical activity enjoyment with average steps, while perceived parental role modeling only significantly mediated the relationship of physical activity self-efficacy with average pedometer steps. Compared to perceived parental support, it seems that perceived parental role modeling had more effect on children's average pedometer steps. 


\section{Figure 7}

$\underline{\text { Pedometer Steps }} \underline{\text { Models }(N=133)}$

\section{Model A Parental Influence}

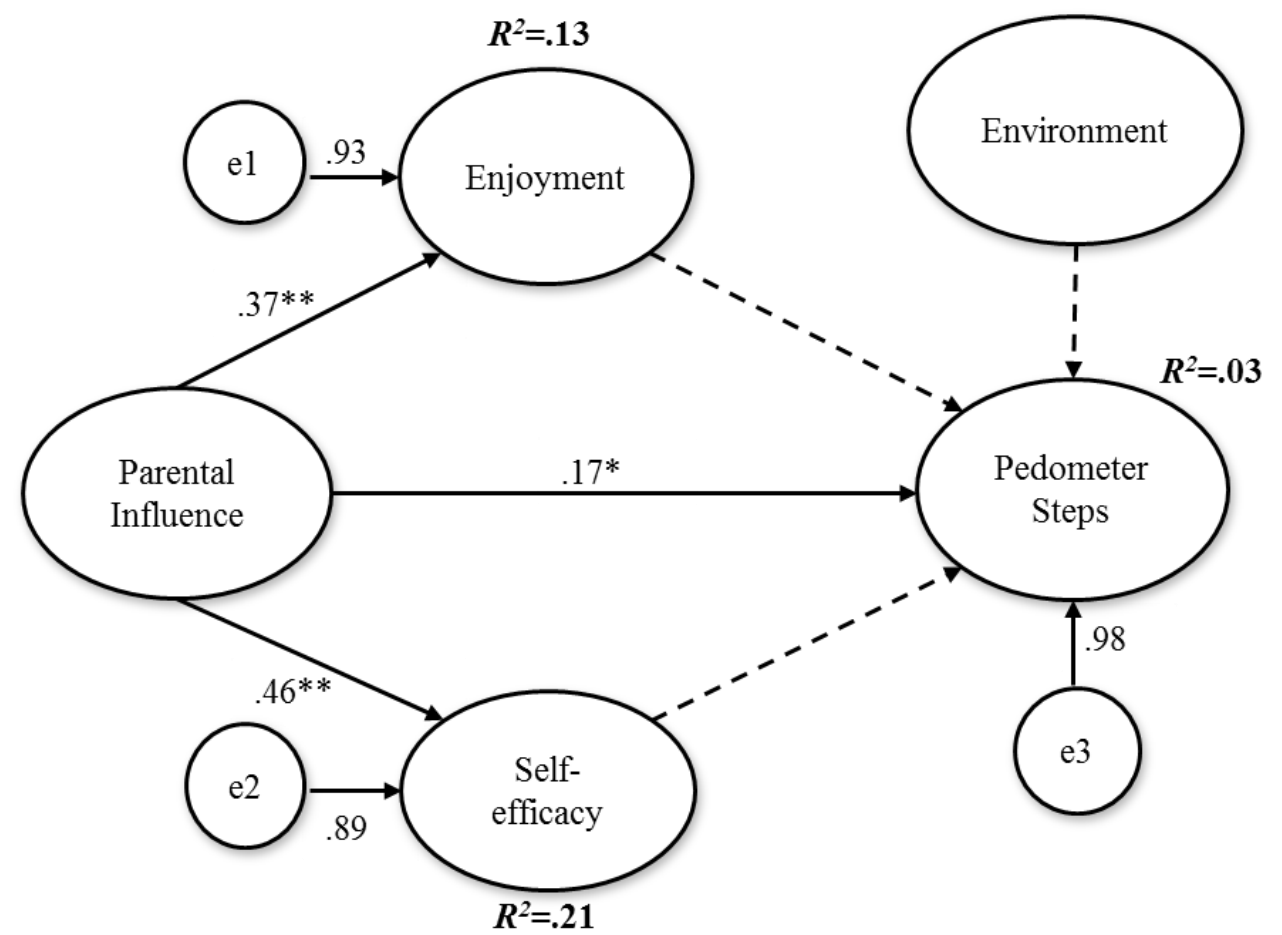

\section{Model B Parental Influence}

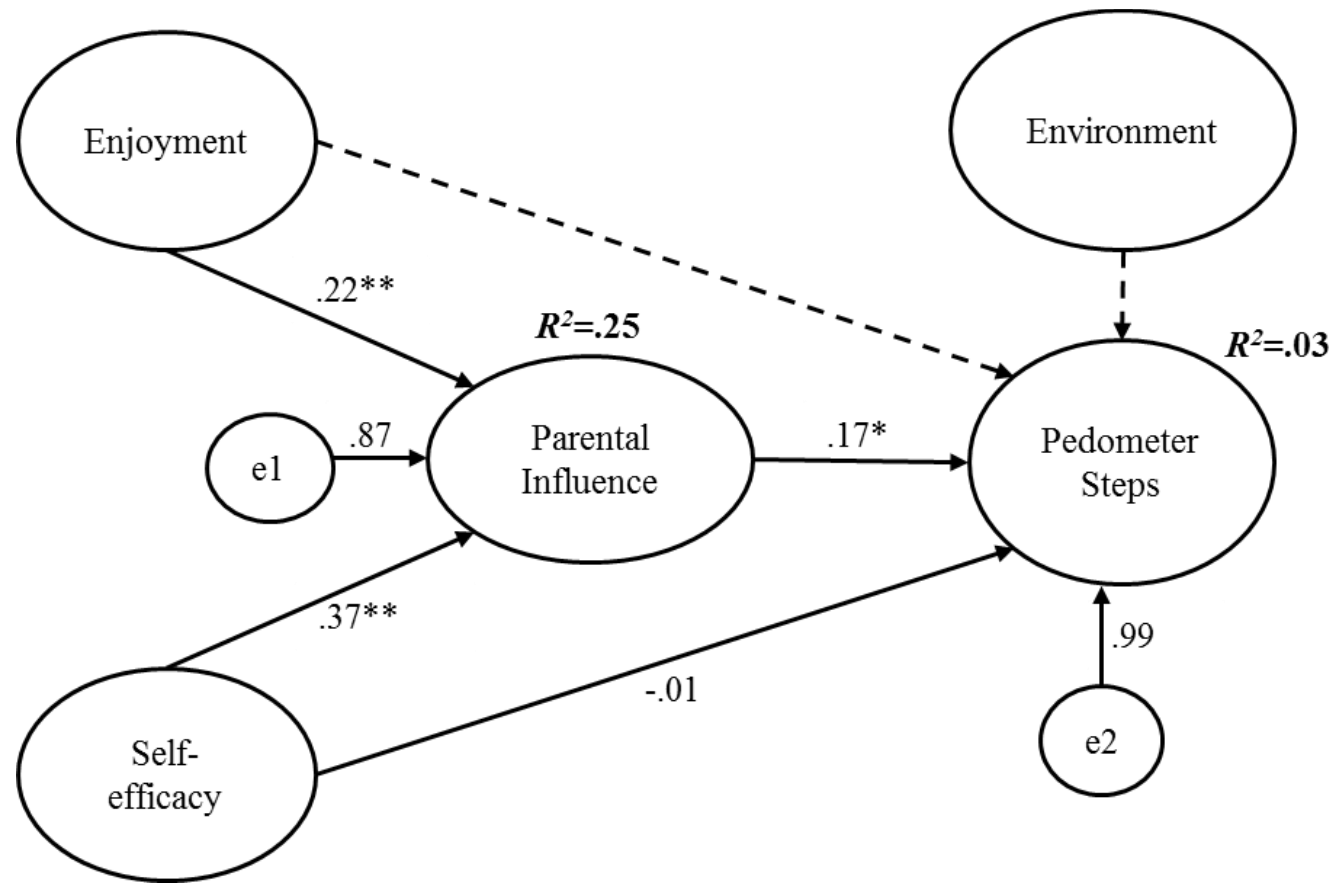




\section{Model C Parental Support}

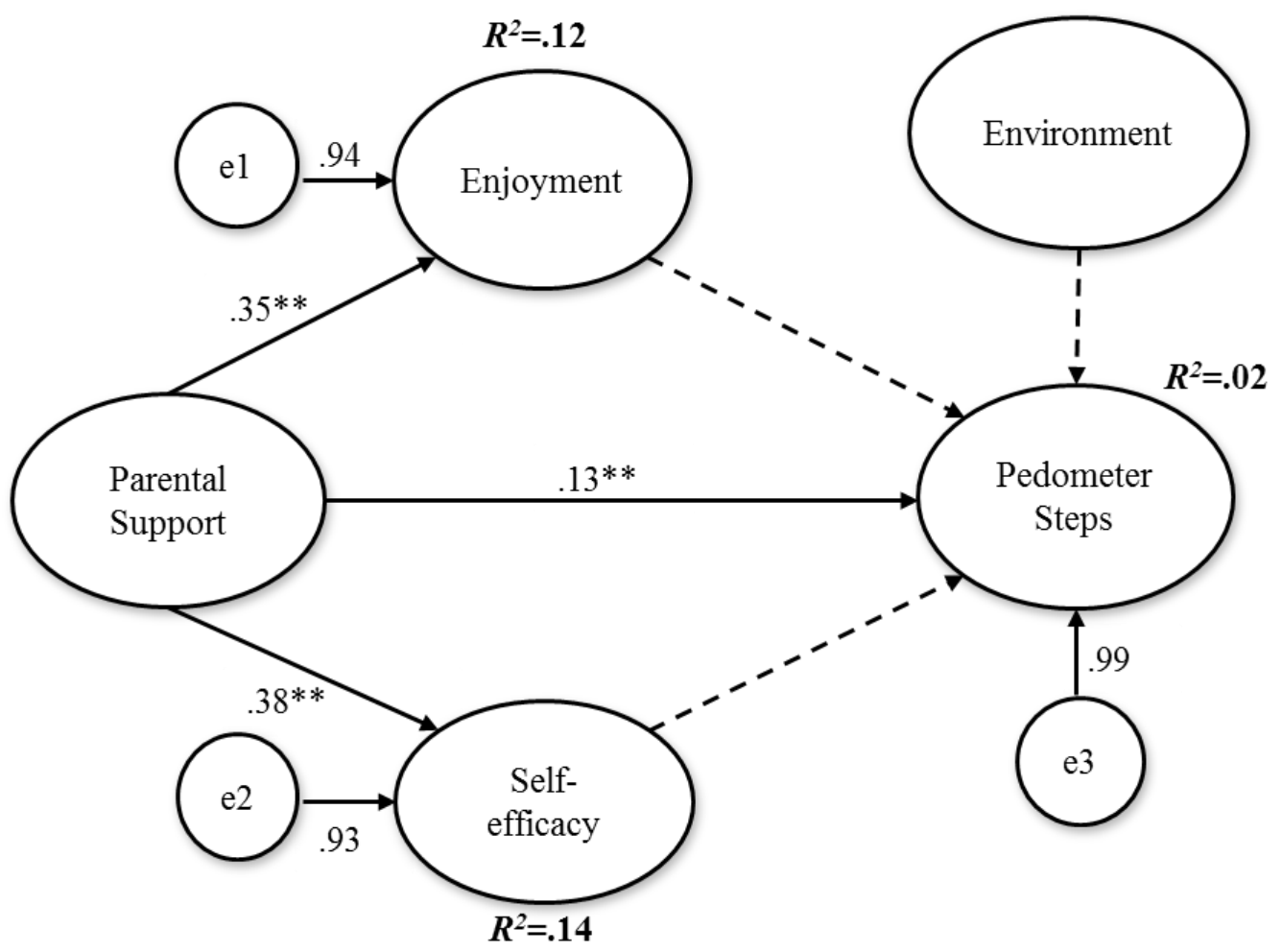

\section{Model D Parental Support}

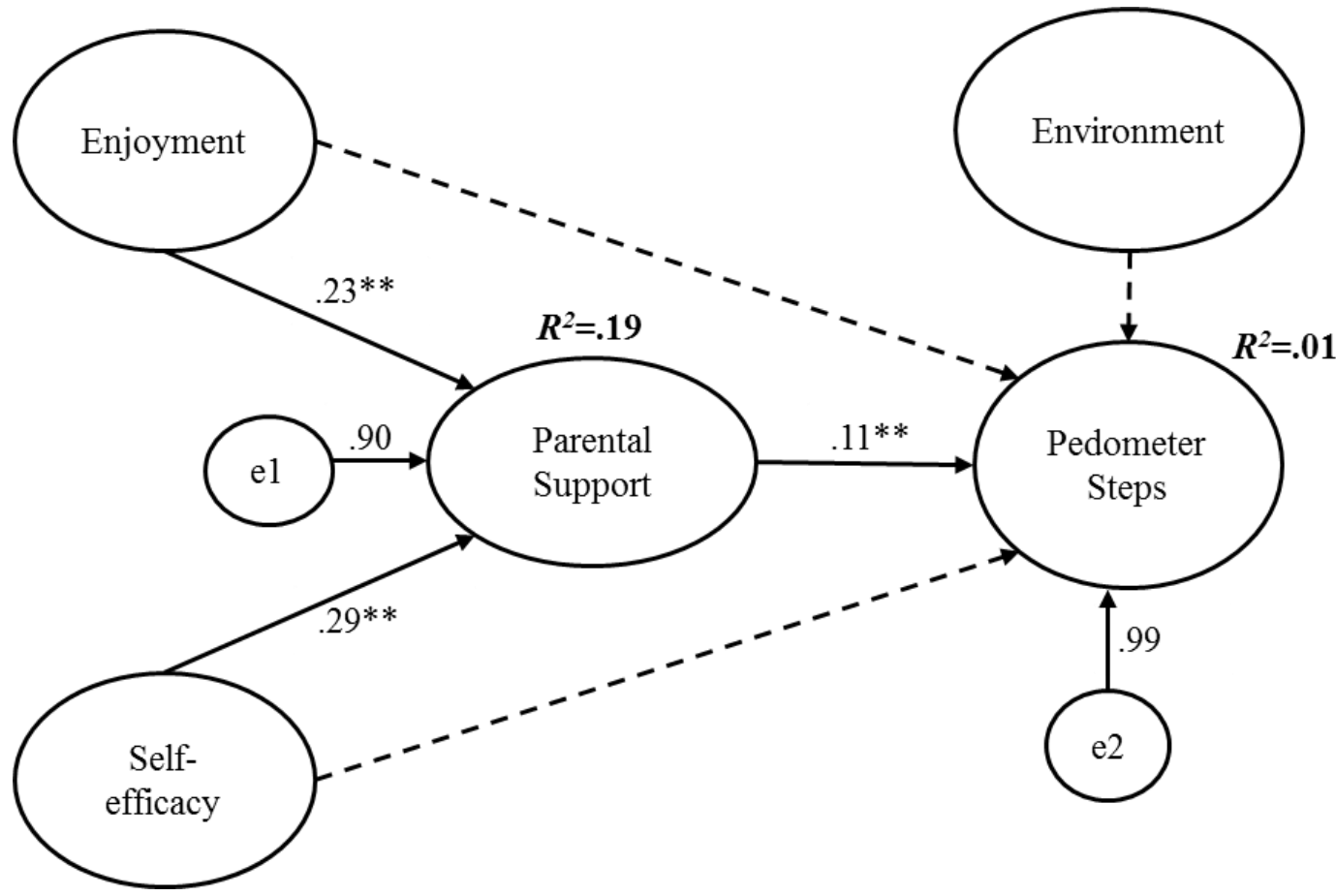




\section{Model E Parental Role Modeling}

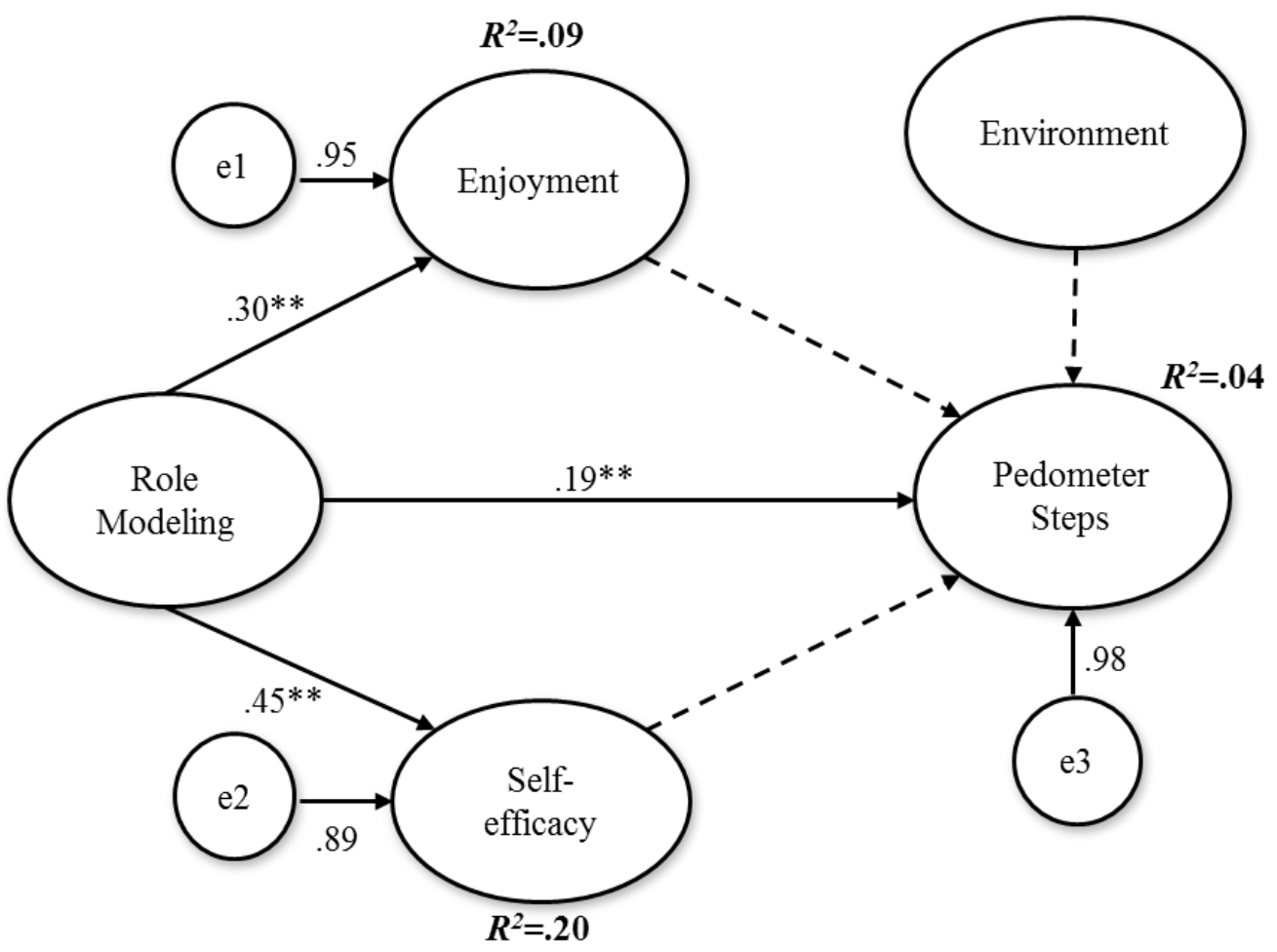

\section{Model F Parental Role Modeling}

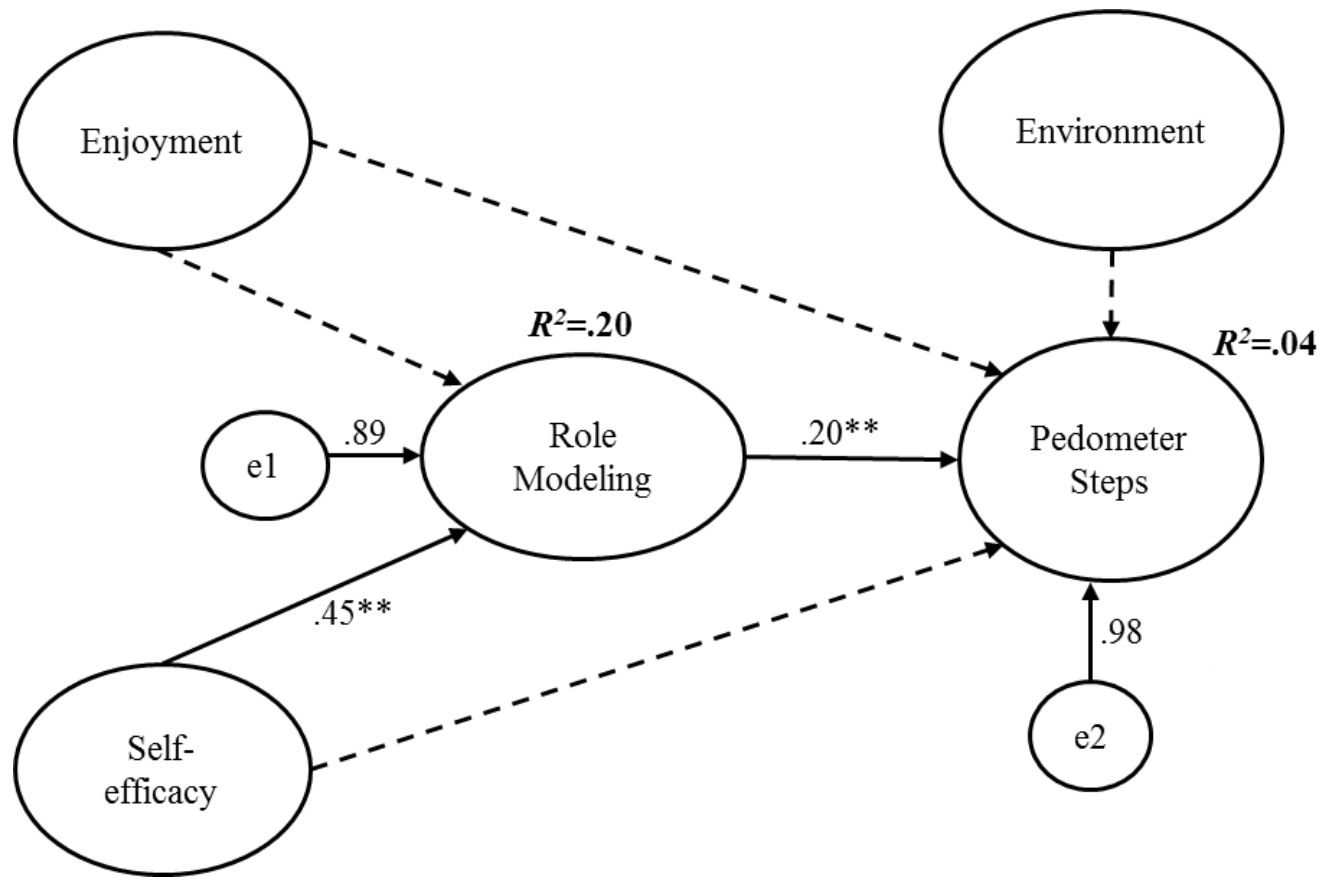

Note. ${ }^{*} p<.05,{ }^{*} p<.01$. Model $\mathrm{B}, \mathrm{D}$, and $\mathrm{F}$ were selected as the final models for average pedometer steps 


\section{Table 16}

Model Fit Indices of Theoretical Model, Model A, Model B, Model C, Model D, Model E, and Model F $(N=133)$

\begin{tabular}{|c|c|c|c|c|c|c|c|c|}
\hline \multirow[b]{2}{*}{ Fit Index } & \multirow[b]{2}{*}{ Cut Point } & \multirow[b]{2}{*}{ Theoretical } & \multicolumn{2}{|c|}{ Parental Influence } & \multicolumn{2}{|c|}{ Parental Support } & \multicolumn{2}{|c|}{ Role Modeling } \\
\hline & & & Model A & Model B & Model C & Model D & Model E & Model F \\
\hline Chi-square test & $P>.05$ & .0004 & .0073 & .40 & .0023 & .7384 & .0021 & .8241 \\
\hline Hoelter Critical N & $>200$ & 60 & 92 & 764 & 76 & 1402 & 75 & 11045 \\
\hline \multicolumn{9}{|l|}{ Parsimony Fit } \\
\hline AGFI & $>.95$ & .98 & .99 & 1.00 & .99 & 1.00 & .99 & 1.00 \\
\hline RMSEA & $<.06$ & $\begin{array}{c}.19 \\
(.11-.28)\end{array}$ & $\begin{array}{c}.15 \\
(.07-.24)\end{array}$ & $\begin{array}{c}0 \\
(0-.21)\end{array}$ & $\begin{array}{c}.16 \\
(.09-.25)\end{array}$ & $\begin{array}{c}0 \\
(0-.12)\end{array}$ & $\begin{array}{c}.17 \\
(.09-.25)\end{array}$ & $\begin{array}{c}0 \\
(0-.13)\end{array}$ \\
\hline Bentler CFI & $>.95$ & .88 & .86 & 1.00 & .77 & 1.00 & .80 & 1.00 \\
\hline
\end{tabular}

Note. Model B, D, and F were the best models. 


\section{Summary of Key Findings}

All the self-reported scales used in this study had good internal consistency reliability with Cronbach's alpha coefficients ranging from .51 to .88 , and good test-retest reliability with ICCs ranging from .61 to .84. Results from the CFA did not confirm the factorial structures of the PASES, PACES, and PAIS. The exploratory factor analysis suggested a three-factor structure for the PASES, two-factor structure for the PACES, PAIS, and perceived environment scale.

In this study, about $87 \%$ of the children returned their pedometers after one week. Approximately $62 \%$ wore pedometers for more than three days, and only $5 \%$ did not wear their pedometers at all. The pedometer steps did not change over time, and the day

of the week selected to start recording pedometer steps did not have significant influence on children's pedometer steps. Wearing pedometers did not have any significant influence on children's perceptions of physical activity self-efficacy, physical activity enjoyment, parental influence and environment.

For most of the days during school year, approximately $82 \%$ of the children went to school by car or school bus, and only $18 \%$ walked or bicycled to school. On average, children spent about one hour a day on TV/DVDs, video games and computers, and took about 7,868 pedometer steps each day. In the study sample, nearly half of the children were overweight or obese, but only $13 \%$ of the children met physical activity recommendations. Based on the data reported by parents, $90 \%$ of the children met the screen-viewing time recommendation.

No relationship was found between screen-viewing time and physical activity levels. Children with a part-time employed or unemployed mother, or who walked to 
school, took more steps each day. Children with a full-time employed father or mother, or with a mother who did not receive any college education, reported to participate in lower physical activity levels.

Overweight or obese children had fewer pedometer steps, and fewer days of meeting physical activity recommendations than non-overweight children. Children's BMI-percentile was influenced by mother's education level and children's perceptions of physical activity self-efficacy. Older Hispanic children with a part-time employed or unemployed father, or with a mother who did not receive any college education were more likely to be overweight or obese.

Compared to African American children, mixed-race children reported to receive more parental influence on physical activity. Children from families with an annual family income less than $\$ 20,000$ had highest physical activity enjoyment. Children with an unemployed or part-time employed mother reported to receive more parental influence on physical activity than children with a full-time employed mother.

Self-reported physical activity levels were significantly related to physical activity self-efficacy, physical activity enjoyment, parental influence and environment, but not significantly related to average pedometer steps. Physical activity self-efficacy, physical activity enjoyment, parental influence and environment were significantly related to each other.

For self-reported physical activity levels, perceived parental influence significantly partially mediated the relationship of physical activity self-efficacy and physical activity levels, while perceived parental support significantly mediated the relationships of physical activity self-efficacy and physical activity enjoyment with 
physical activity levels. Perceived parental role modeling had indirect effect on selfreported physical activity levels through physical activity self-efficacy and physical activity enjoyment. Both physical activity enjoyment and physical activity self-efficacy had significant direct effect on self-reported physical activity levels, but physical activity enjoyment had stronger effect on physical activity levels compared to physical activity self-efficacy. The parental influence model, parental support model, and parental role modeling model could explain about $30 \%, 31 \%$, and $22 \%$ of the variance of self-reported physical activity levels respectively.

For pedometer steps, only perceived parental influence, parental support, and parental role modeling had significantly direct effect on average pedometer steps. Perceived parental influence and parental support partially mediated the relationships of physical activity self-efficacy and physical activity enjoyment with average pedometer steps, while perceived parental role modeling mediated the relationship between physical activity self-efficacy and average pedometer steps. Compared to physical activity enjoyment, physical activity self-efficacy had stronger indirect effect on average pedometer steps. The parental influence model, parental support model, and parental role modeling model could account for about $3 \%, 1 \%$, and $4 \%$ of the variance of average pedometer steps. For both self-reported physical activity levels and average pedometer steps, the results of hierarchical regression models indicated that only physical activity enjoyment was the significant predictor of physical activity after controlling for demographic factors (See Appendix K). 


\section{CHAPTER V}

\section{DISCUSSION AND CONCLUSIONS}

The purpose of this study was to explore the psychosocial and environmental determinants of physical activity among elementary school children aged 8 to 11 years old attending afterschool programs. A cross-sectional correlational design was used to explore the determinants of physical activity, and a pretest-posttest study was conducted to examine the influence of wearing pedometers on the hypothesized determinants of children's physical activity. A total of 133 children were recruited from ten elementary schools with afterschool programs from August 2013 to October 2013 with all children completing the posttest surveys after wearing a pedometer for one week to monitor physical activity. A subsample of 50 of these children was randomly selected to also complete the pretest surveys prior to receiving pedometers. Six children (one in the pretest-posttest subsample) did not complete the posttest surveys due to a health problem, injury, or family relocation to a different geographical area.

This chapter first discusses this research results related to previous literature findings. Then the implications for further research, practice and policy are presented. Finally, limitations of this study are addressed and a short summary of the conclusions is given.

\section{Discussion}

\section{Scale Construction and Psychometric Characteristics}

Scales measuring physical activity (PAQ-C), physical activity enjoyment 
(PACES), and parental influence (PAIS) had excellent internal consistency reliability with Cronbach's alpha coefficients greater than .80 . The PASES (physical activity selfefficacy) had fair or good internal consistency reliability with a Cronbach's alpha coefficient of .73, which may have been due to the 2-point Likert (yes or no) scale. The internal consistency reliability for the modified environment scale was also acceptable because this scale has only five items.

Considering subscales of the instruments used to measure study variables, all the subscales' internal consistency reliability was acceptable except the positive alternatives component of the PASES. The positive alternatives component items were specifically developed for children, while other two components were adopted from adult physical activity self-efficacy scales (Saunders et al., 1997). In Saunders's study (1997), the Cronbach's alpha coefficient was .54 for the positive alternatives component. All these findings may indicate that children's confidence to perform physical activity is not significantly influenced by their ability to overcome other existing choices such as watching TV, staying at home, or doing other things. Test-retest reliability was good for all the scales measuring the hypothesized determinants of physical activity, with the PASES and PAIS having excellent test-retest reliability (Zaki et al., 2013).

Different from previous study findings, the three-factor structure of the PASES did not fit the data adequately. Originally, the three-factor structure was developed using a principal component factor analysis with Varimax rotation among $3195^{\text {th }}$ grade children, but no confirmatory analysis model was conducted to verify the factorial structure of the PASES (Saunders et al., 1997). Most studies in elementary school children using this scale did not conduct a CFA to verify the factorial structure of this 
scale (Huhman et al., 2007; Thompson et al., 2008). In this study, after deleting two items (Item 1 and 4) due to low item-total correlation coefficients and similar contents with other items, a new three-factor structure was suggested. However, the new three-factor structure still did not fit the data adequately. Therefore, future studies should modify or develop valid physical activity self-efficacy instrument for elementary school children.

The two-factor structure of the PACES in this study was consistent with previous study findings in $5643^{\text {rd }}$ grade children with all positively worded items loading onto one factor, and all negatively worded items loading onto the other factor (Moore et al., 2009). In the same way, the PAIS also had a different two-factor structure with all positively worded items loading onto one factor and all negatively worded items loading onto the other factor, from the original factorial structure (Welk et al., 2003). Another study examined the factorial structure of the PAIS among 601 adolescents, and found that a three-factor structure (role modeling, parental encouragement, and parental support) also did not fit data adequately. Although the new factorial structures of the PACES and PAIS do not fit the data adequately, they do fit better compared to the original factorial structures. The unsatisfied factorial structures of the PACES and PAIS might be due to the negatively worded items that were difficult for children to understand. The problem of including negatively worded items in a measurement for elementary school children was discussed, and suggestions were also provided later.

The exploratory factor analysis of the modified environment scale suggested a two-factor structure, and all items together accounted for about $63 \%$ of the variance of the perceived environment. The CFA model confirmed the factorial structure of this scale. Therefore, this modified environment scale including the school environment had good 
construct validly, and it appears to be a cost-effective tool to assess children's perceptions of their physical activity environment related to equipment accessibility and neighborhood safety.

Including negatively worded items in a scale is recommended for reducing acquiescence response set bias (Nunnally \& Bernstein, 1994). Response set bias occurs when participants respond to all items in the same manner, such as selecting the same answer for all the questions. Consistent with other studies, this study found in the PAIS and PACES that negatively worded items tended to load onto a separate factor rather than contribute uniquely to the construct of the variable (Cordery \& Sevastos, 1993; Fried \& Ferris, 1986). The possible causes include: (1) acquiescence, (2) careless responding, and (3) confirmation bias (Weijters, Baumgartner, \& Schillewaert, 2013). Acquiescence means a preference for the positive or negative side of the rating scale (i.e., the "agree" side or the "disagree" side). Careless responding means that some subjects do not read the item carefully enough and answer a negatively worded item as if it is a positively worded item. Confirmation bias means the phenomenon that occurs when respondents answer a question with beliefs being activated that are consistent with the way the question is stated. These beliefs carry over and influence responses to other items (meaning negatively worded items are not marked the way they would have the beliefs not carried over). Especially with children as participants, both acquiescence and careless responding could be operating. In addition, education levels were suggested as one contributor to this issue because participants with low levels of education might not be capable of recognizing the reverse nature of the items (Fried \& Ferris, 1986). Therefore, it was proposed that using negatively worded items in young children was not appropriate 
considering their cognitive development level (Marsh, 1984). During data collection in this study, children complained of difficulty understanding the negatively worded items. Further studies should examine the readability of negatively worded items among elementary school children, and compare the reliability and validity between a scale with negatively worded items and a scale without negatively worded items. Excluding negatively worded items when developing self-report scales for elementary school children should be considered.

The majority of the children in this study did not have difficulty understanding the 4-point or 5-point Likert scales. Modifying the 2-point Likert scale to a 5-point Likert scale format might improve the internal consistency reliability of the PASES. During data collection, some children preferred a 'no opinion' choice when they could not make a decision on agreement or disagreement. Especially for the PAIS, some children mentioned that their father and mother had very different physical activity behaviors such as one was very active, and the other one was not active at all. Thus, using two separate scales (one for father and one for mother) to assess parental influence might be more valid. This study did not have a very large sample size, which could be one explanation for the poor fit of the factorial structures of the PASES, PACES and PAIS to the study data. Evidence suggests that when each component of a scale has at least 5 items, a sample size of 200 is recommended for factor analysis (Mundfrom, Shaw, \& Ke, 2005). Therefore, a large sample size is needed to further examine the factorial structures of these scales among elementary school children. 


\section{Compliance Rate, Reliability, and Reactivity of Wearing Pedometers}

Based on the findings in this study, wearing pedometers for four consecutive days resulted in a reliability coefficient greater than .80 , and wearing pedometers for two consecutive days yielded a coefficient greater than .70 . Other researchers have found that the intraclass correlation coefficient (ICC) exceeded .80 for three consecutive days, and exceeded .70 for just two consecutive days among children aged 5 to 19 years old (Craig et al., 2010). One study in U.S. adolescents suggested that six consecutive days obtained reliability coefficient greater than .80 (Rowe et al., 2004). Currently, most studies use seven consecutive days to assess children's physical activity levels (Clemes \& Biddle, 2013). Considering the compliance rate and cost of wearing pedometers for seven consecutive days, the findings of this study suggest that four consecutive days of wearing pedometers is adequate to obtain an accurate estimation of children's physical activity levels. In order to include children's physical activity levels on the weekend, three weekdays and one weekend day are recommended to obtain a better estimation of elementary school children's physical activity levels.

In this study, $87 \%$ of the children returned their pedometers, and about $38 \%$ wore pedometers for the full seven days. Compared to a Canadian study in which $58 \%$ returned pedometers and data, and $12 \%$ returned pedometers but not data (Craig et al., 2010), the rate of returning pedometers in this study was encouraging. In the Canadian study, a foldable flying disc was awarded to children who completed the study. In this study, basketball prizes and playground balls served as external motivation for children to return pedometers and pedometer logs. Some children in this study complained that they would have preferred a bag of candy (poor alternative for health promoting study) instead of a 
playground ball because they already had many balls at home or they did not like playing ball. One girl who won a basketball prize asked the investigator if she could change it to a soccer ball instead, because she already had a basketball at home.

Some researchers indicated that providing monetary incentives over the course of study was more effective than gifts or prizes to recruit and retain adolescents in studies (Martinson et al., 2000). For elementary school children aged 8 to 11 years old, gift certificate, DVDs, tickets to concerts or sporting events, t-shirts, and movie pass have been suggested based on their developmental level (Rice \& Broome, 2004). However, no study was found examining the effectiveness of using incentives of any sort on recruiting and retaining elementary school children in studies. From this study, the investigator concluded that sports equipment motivated most children to participate in the research study, but it would be more effective if more variety in types of sports equipment were available.

Another factor that might affect compliance rate is the availability of adult participation or guidance. Evidence suggests that involving children and school staff directly in the study process could yield higher response rates in school-based surveys than involving parents and school staff or only school staff (Claudio \& Stingone, 2008). In this study, the investigator visited the afterschool programs each weekday to help children record pedometer steps, reset pedometers to zero, and remind them to wear pedometers if they had forgotten. As a result, about 93 (69.9\%) children wore pedometers on weekdays, and this number decreased to $74(55.6 \%)$ on weekends. Therefore, it was concluded that investigators' daily physical presence might markedly improve the compliance rate of wearing pedometers among elementary school children. In future, 
afterschool program staff might play the role of investigators to help children record pedometer steps, reset pedometers to zero, and remind them to wear pedometers.

Reactivity occurs when study procedure influences individuals' behaviors (Rowe et al., 2004). The reactivity of using pedometers to assess children's physical activity levels is that children may take more steps than their normal levels during the initial study period. Different from this study's findings, other study found significant differences among the seven-day pedometer steps, although no significant differences were found between two consecutive days (Craig et al., 2010). One study in 1,115 New Zealand children found that children took more steps on weekdays compared to weekend days (Duncan, Schofield, \& Duncan, 2006). However, this study did not find any differences between weekday pedometer steps and weekend pedometer steps. Similar to the findings of this study, a study in $1094^{\text {th }}-6^{\text {th }}$ grade children found no evidence of the reactivity of wearing pedometers, and also no influence of children's previous knowledge of pedometers on their reactivity of wearing pedometers (Prewitt, Hannon, \& Brusseau, 2013). Although there is controversy about the reactivity of wearing pedometers, this study's findings suggest that there is no reactivity of wearing pedometers among children aged 8 to 11 years old. During data collection, the investigator repeatedly emphasized that each child would receive a playground ball no matter how many steps he/she took. In addition, most children in this study reported wearing pedometers on other occasions, and pedometers were not considered new, exciting, or innovative anymore. Previous studies have found evidence of reactivity of wearing pedometers, which may have resulted from the high percentage of missing data (Craig et al., 2010), missing data imputation (Rowe et al., 2004), or statistical methods (repeated measures ANOVA vs. mixed linear 
regression model). In addition, the day of the week selected to start recording pedometer steps did not have significant influence on children's pedometer steps in this study.

Although no evidence of the reactivity of wearing pedometers in this study sample was found, attaching the pedometers to overweight or obese children's pants was very difficult because most children in this study did not wear a belt. Most of the time, overweight or obese children's pedometers turned upside down or at an angle instead of remaining vertical because of increased waist circumference. Pedometers are known to often have a higher percentage of error for overweight or obese children than healthy weight children (Mitre, Lanningham-Foster, Foster, \& Levine, 2009). Using pedometers that can attach to children's shoes or using pedometer belts may be more practicable for overweight or obese children. Compared to pedometers, some scientists suggest using accelerometers to accurately estimate overweight or obese children's physical activity levels, because accelerometers can respond both to vertical and horizontal acceleration of the ankle (Mitre et al., 2009).

\section{Sample Characteristics}

Compared to the general population of the two townships that were the settings for this study, this study sample had higher percentages of Hispanics and African Americans. Similarly, families in this study had lower annual family income, and higher percentage of unemployment compared to the general population of the two townships. Marital status and education levels in the study sample were very similar to the general population.

Though the study sample was not representative of the population in the two townships, it was relatively representative of the ethnic/race characteristics of children 
attending afterschool programs. Ethnic minority children are more likely to attend afterschool programs. In 2009, about 24\% of African-American, and 21\% of Hispanic children attended afterschool programs compared to the national average of $15 \%$ (Afterschool Alliance \& JCPenny Afterschool, 2009). Ethnic minority children are experiencing multiple disparities in health and healthcare, and are more likely to suffer from obesity, hypertension and other health problems while also participating in lower levels of physical activity and higher levels of sedentary activity (Flores \& Lin, 2013; Singh, Kogan, Van Dyck, \& Siahpush, 2008). Therefore, afterschool programs play a very important role as optimal sites for promoting health and healthy behaviors for disadvantaged children.

\section{Obesity Status}

Nearly half of the children in this study were overweight or obese, with $19 \%$ classified as overweight and 30\% were obese. Analysis of the NHANES data found that about $39 \%$ of children aged 6 to 11 years old were overweight or obese in 2008, and the prevalence or being overweight or obese was disproportionately high in African American and Hispanic children (Ogden, 2010). In 2011, about 32\% of the children in Indiana were overweight or obese (Kaiser Family Foundation, 2011a). The low socioeconomic status, parents' low education levels, and high percentage of minority children may have contributed to the higher percentage of overweight or obesity in this study sample. Hispanic children in this study were more likely to be overweight or obese than non-Hispanic children (prevalence: $56 \%$ vs. $47 \%$ ). In this study, about $38 \%$ of African American children were overweight or obese compared to the national average of 41\% in African American children (Singh, Siahpush, \& Kogan, 2010). 
Similar to previous study findings (Hills et al., 2011), overweight or obese children in this study had lower levels of physical activity than non-overweight children. However, children in this study who reported participating in higher levels of physical activity were more likely to be overweight or obese. Evidence suggests that indirect measures usually overestimate children's physical activity levels (Guthold, Cowan, Autenrieth, Kann, \& Riley, 2010). Overestimation may be one explanation for the controversial relationship between self-reported physical activity levels and odds of being overweight or obese. The self-reported physical activity scale used in this study was a seven-day recall survey, so recall bias might have existed that influenced the relationship between physical activity levels and odds of being overweight or obese. A study in England among adults found that overestimation of physical activity levels was associated with lower BMI (Watkinson et al., 2010), but no study was found in children that examined the influence of overestimation of physical activity levels on BMI. Further studies are needed to examine the under or overestimation of physical activity levels among children when using self-reported physical activity scales.

Children in this study with unemployed fathers or fathers working only part-time were 12 times more likely to be overweight or obese, and children of mothers with no college education were 5 times more likely to be overweight or obese after controlling for other demographic factors. In other words, father's employment status and mother's education level had a very strong influence on children's obesity status. Similar to national patterns, children in households with lower education and income levels, and higher unemployment were more likely to be overweight or obese, and this relationship was stronger in 2007 compared to 2003 (Singh et al., 2010). Another study found that a 
child with a full-time employed mother during mid-childhood was more likely to become overweight at age 16 (Stephanie von Hinke Kessler Scholder, 2008). Although this study did not find an association between mother's employment status and children's obesity status, the fathers' employment status was strongly related to children's obesity status. One possible explanation could be that fathers are the primary contributors of income to the family, while mothers are the primary caregivers and cooks for a family. Mother's education levels may influence their parenting, role modeling and support to their children. Evidence indicates that full-time workers participate in higher levels of physical activity than unemployed or part-time employed workers (Van Domelen et al., 2011).

Due to unemployed or part-time employed fathers' lower physical activity levels, children may be negatively affected by their father's unhealthy behaviors resulting in lower levels of physical activity. All these findings call attention to the vulnerable children of unemployed or part-time employed fathers, and mothers with low education levels.

\section{Physical Activity}

The majority of the children (90\%) in this study, more than the national average (79\%), viewed TV/DVDs, videos or computers for no more than 2 hours a day. These data were from parents, and parents may have underestimated the screen-viewing time of their children such as the screening-viewing time in afterschool programs. Another possible explanation is that parents might carelessly read the question as how many minutes per day instead of per week even though the words "per week" were bolded and with a bigger font. The investigator realized this wording problem during data collection, but only about one quarter of the data were rechecked with parents or children due to 
time limitations. For future studies, the short time span (per day) is suggested to assess children's screen-viewing time to avoid careless reading. All children enrolled in $3^{\text {rd }}$ grade to $12^{\text {th }}$ grade in the Greater Clark County school system were participating in a new computer program since this current school year (White, 2013). Each child received a free laptop computer, apart from $\$ 12$ fee for insurance. Due to this new computer program, children spent the majority of their afterschool time playing computer games, after finishing homework with the help of the afterschool program teachers. Further studies are needed to examine the influence of a computer program on children's academic performance, physical activity, and obesity status. This new computer program might be one confounder that influenced children's physical activity and sedentary activity levels in this study.

Only $13 \%$ of the children in this study met physical activity recommendations of $13,000 \mathrm{steps} /$ day for boys and 11,000 steps/day for girls. Analysis of the national data found that about $70 \%$ of children reported meeting recommended levels of physical activity (Fakhouri et al., 2013), and 42\% of children met recommended levels of physical activity when using accelerometers to assess physical activity levels (Troiano et al., 2008). Worldwide, about $24 \%$ of boys and $15 \%$ of girls meet physical activity recommendations (Guthold et al., 2010). The low percentage of children in this study meeting physical activity recommendations may be due to demographic characteristics of this vulnerable population, or the inconsistent cut points of the transformation from pedometer steps to moderate- or vigorous-intensity physical activity. It appears logical to conclude that children in this study participated in the afterschool programs due to parents' busy work schedules, children's learning difficulties and need for afterschool tutoring, or factors 
related to low socioeconomic status, thus, they might be more likely to have lower levels of physical activity and higher rates of overweight or obesity. Afterschool programs aim to provide children academic support rather than opportunities for physical activity. Therefore, most opportunities for physical activity might occur in school time for this study sample, and afterschool time may be a primary targeting opportunity for future studies to improve physical activity levels in this group of children.

Regarding the decision on what number of pedometer steps is needed to decide recommended levels of physical activity, Tudor-Locke (2011) suggested cut points of 13,000 steps/day for boys and 11,000 steps/day for girls. A recent study reexamined these cut points for physical activity recommendations, and the findings suggested that 11,500 accelerometer steps per day and 9,000 pedometer steps per day for both boys and girls provided a reasonable estimation of activity equivalent to 60 minutes of moderate- to vigorous-intensity physical activity per day (Adams, Johnson, \& Tudor-Locke, 2013). When using 9,000 pedometer steps/day as the cut point, about $30.1 \%(n=40)$ of the children in this study met physical activity recommendations. Considering the nonconsistent cut points, more studies with larger sample sizes are needed to further examine the cut points of using pedometer steps to assess American children's status of meeting physical activity recommendations.

On average, children in this study took about 7,868 pedometer steps per day, with 8,441 steps for boys and 7,197 steps for girls. On average, U.S. boys take about 13,000 accelerometer steps per day, and girls take about 12,000 accelerometer steps per day (Tudor-Locke, Johnson, \& Katzmarzyk, 2010), somewhat higher than levels among Canadian children, 12,037 pedometer steps for boys and 10,587 pedometer steps for girls 
each day (Craig, Cameron, \& Tudor-Locke, 2013). A study in 608 children from two Mid-Western communities in U.S. found that children took an average of 11,665 pedometer steps, with 12,709 steps for boys and 10,834 steps for girls (Eisenmann et al., 2007). Thus, children in this study had much lower physical activity levels than children in the U.S. or Canada or even compared to other Midwestern communities. Seasonal variation may be a confounding factor influencing children's physical activity levels in this study. Data in this study were collected from August 2013 to October 2013. The mean temperature was $74.9^{\circ} \mathrm{F}$ (range: $52^{\circ}-96^{\circ}$ ) in August, $69.5^{\circ} \mathrm{F}$ (range: $48^{\circ}-96^{\circ}$ ) in September, and $54.6^{\circ} \mathrm{F}$ (range: $26^{\circ}-82^{\circ}$ ) in October (National Weather Service Weather Forecast Office, 2013). Evidence suggests that children tend to take more steps in Spring (February) compare to Winter (May) (Beighle, Alderman, Morgan, \& Le Masurier, 2008). The rolling enrollment method due to limited funding support used for data collection in this study makes it impossible to avoid the seasonal variation. Future studies should consider the confounding factor weather when assessing children's physical activity levels, especially in intervention studies. Based on the pedometer steps' cut points for boys and girls, about $60 \%$ of boys and $53 \%$ of girls were classified as sedentary in this study, but based on the data reported by parents, about $90 \%$ of the children met the screen-viewing time recommendation. These results also point out that parents may underestimate their children's screen-viewing time, or may carelessly read the question as how many munities per day instead of per week.

Patterns of transportation from home to school were another factor in this study that had a significant influence on children's average pedometer steps. Unsurprisingly, children who walked to school had the highest number of pedometer steps. These results 
are consistent with a study in Australia showed that children who walked to school had more steps on weekdays but not on weekend days (Trapp et al., 2013). Almost half of the children in this study went to school by car, consistent with previous study findings in the U.S. (Trapp et al., 2013). Although getting to or from school provides the most universal opportunity for children to participate in incidental physical activity (Committee on Environmental Health, 2009), only about $19 \%$ of the children in this study walked or rode a bicycle to school. A WHO study among 34 countries other than the U.S. found that the percentages of boys who walked or bicycled to school ranged from $23 \%$ in the Cayman Islands to $83 \%$ in China, and the percentages for girls ranged from $11 \%$ in United Arab Emirates to $86 \%$ in China (Guthold et al., 2010). Built environment plays an essential role in children's transportation patterns from home to school. Therefore, this study suggests that a more supportive and safer environment should be constructed to facilitate children to walk or bicycle to school.

\section{Obesity Status and Physical Activity}

As discussed above, although this study did not find a significant relationship between BMI percentile and pedometer steps, it did find that children's BMI and BMI zscores were negatively correlated with pedometer steps. In addition, overweight or obese children took fewer pedometer steps, and had fewer days of meeting physical activity recommendations compared to non-overweight children. Similarly, a study in 608 children found that BMI was negatively correlated with pedometer steps, and children not meeting physical activity recommendations were two times more likely to be overweight or obese (Eisenmann et al., 2007). However, the negative correlation between physical activity levels and BMI was found in only five out of 17 studies, mostly in studies when 
using pedometers or accelerometers to assess children's physical activity levels (PrenticeDunn \& Prentice-Dunn, 2012).

This study also did not find a significant relationship between screen-viewing time and BMI based on the data reported by parents. Although most previous studies indicate that there is a positive correlation between sedentary time and BMI, no significant correlation is found when using accelerometer or direct observation methods to assess children's sedentary activity levels (Prentice-Dunn \& Prentice-Dunn, 2012). Therefore, it is recommended to further examine the relationships between physical activity, sedentary activity and BMI by using objective methods to assess physical activity and sedentary activity levels.

Considering the relationship between physical activity and obesity status, evidence suggests that pedometer steps are more strongly correlated with body fat than with BMI (Duncan et al., 2006). Moreover, a three-year longitudinal study in obese children indicates that physical activity change is associated with BMI change over time, but that the reduction in BMI is small even for the greatest increase in physical activity (Trinh, Campbell, Ukoumunne, Gerner, \& Wake, 2013). Therefore, this study suggests that physical activity may have more influence on children's body composition than on BMI or losing weight when considering children's body growth characteristics. Moreover, when examining the relationship between physical activity and obesity status, more reliable and valid measurements of body composition are needed for future studies.

Many methods have been developed to assess obesity status among children. These methods can be categorized as direct measures or indirect measures. Direct measures, such as densitometry (underwater weighing) and scanning using dual energy $\mathrm{x}$ - 
ray absorptiometry, are more accurate than indirect measures but expensive, time consuming and not as practical, especially for large epidemiological studies (Kipping et al., 2008). Indirect measures, such as waist circumference, skin fold thickness, and body mass index (BMI), do not precisely assess children's body composition (percent body fat), and there is considerable variation across age, sex, and ethnicity (Dehghan \& Merchant, 2008). BMI has been recommended as a method to screen for childhood obesity in clinical practice and epidemiology (Moyer et al., 2005). However, the ability of BMI to assess children's obesity status differs between population groups, and there is argument on using national BMI reference standards or international BMI reference standards (Reilly, 2002). In addition, using BMI to assess children's obesity status has high specificity (95-100\%), but low sensitivity (36-66\%). By contrast, bioelectrical impedance analysis (BIA) was found to be a reliable, inexpensive, and practical noninvasive indirect method of assessing body composition (percent body fat, percent lean body mass and percent body water) with minimal variability (Dehghan \& Merchant, 2008). Though BIA is inexpensive compared to other direct methods, it was still beyond the funds available for this study. Nevertheless, BIA has the potentiality to be useful in screening for childhood obesity in large epidemiology and studies in the future.

Though many methods have been developed to assess children's obesity status, more studies are needed to evaluate the specificity and sensitivity of each method. One study in Chinese children indicates that population-specific measures have higher sensitivity and lower specificity than international cutoff values of BMI (Fu et al., 2003). Therefore, to avoid underestimation of the prevalence of childhood obesity, further studies are needed focusing on exploring population-specific measures (or cutoff values). 
Reilly (2002) indicated that it was premature and might be clinically harmful to use a universal definition of childhood obesity now, thus there is a need to explore a more complex approach to defining childhood obesity rather than the simpler universal approach now used most widely.

\section{Determinants of Physical Activity}

Parental influence. The positive relationship between physical activity and children's perceived parental influence was supported by the findings of this study and is congruent with other studies (Beets, Cardinal, et al., 2010). However, only a few studies have examined the influencing mechanisms of perceived parental influence on children's physical activity (Trost et al., 2003). Though some researchers suggest that physical activity self-efficacy, attraction to physical activity, or perceived competence mediate the relationship of parental influence with physical activity; no researcher has suggested that parental influence may be the mediator. This is the first study finding evidence that perceived parental influence partially mediates the relationships of physical activity selfefficacy and physical activity enjoyment with physical activity (Figure 5 Model B and Figure 6 Model B). Children are not independent agents. Even though they have very high confidence and intention to perform physical activity, they may not have access to exercise facilities due to lack of transportation, or they may lack parental encouragement. Parental influence including support and role modeling may bridge the gap between children's intention and behavior. The Cognitive Dissonance Theory in social psychology may provide another explanation of the mediating role of perceived parental influence between physical activity self-efficacy, physical activity enjoyment, and physical activity (Festinger, 1962). This theory proposes that people want to reduce the dissonance 
between beliefs that contradict with one another, or people want to be consistent with their beliefs (Festinger, 1962). Evidence has suggested that the drive to reduce cognitive dissonance emerge without the need for much life experience, even in preschoolers (Egan, Santos, \& Bloom, 2007). Children who have high physical activity self-efficacy and physical activity enjoyment levels may perceive their parents as supporting them participating in physical activity because this will be consistent with their own beliefs.

Different from the YPAP model, and other study findings, this study found that perceived parental support had direct effects on self-reported physical activity levels, and mediated the relationships of physical activity self-efficacy and physical activity enjoyment with self-reported physical activity levels (Figure 5 Model D). A study in 174 children indicated that parents' self-reported support had indirect influence on children's self-reported physical activity levels through children's physical activity self-efficacy (Leary, Lilly, Dino, Loprinzi, \& Cottrell, 2013). Similarly, another study in 418 children found that parents' self-reported support had only indirect effect on children's selfreported physical activity levels through children's physical activity self-efficacy (Trost et al., 2003). Unlike these two studies, children in this study completed the PAIS measuring parental influence. In other words, parental influence is children's perceptions in this study rather than parents' perceptions. Children's perceptions of parental support may be different from parents' perceptions of support. In other words, parents may perceive that they have provided support for their children to participate in physical activity, but children may not realize or may not actually receive support. Further studies are needed to explore how children's perceptions of parental support differ from parents' 
perceptions of support in child-parent dyads, and how these supports influence children's physical activity levels.

Perceived parental support, including parental encouragement, parental involvement, and parental facilitation, seems to have more influence on children's selfreported physical activity levels (Figure 5 Model D), while perceived parental role modeling had more influence on children's average number of pedometer steps (Figure 6 Model F). Some evidence indicates that parental role modeling, measured by parental self-reported physical activity levels, does not have direct effect on children's selfreported physical activity levels (Trost et al., 2003). This may be explained by this study finding that perceived parental role modeling had indirect effect on self-reported physical activity levels through physical activity self-efficacy and physical activity enjoyment (Figure 5 Model E). However, a family-based intervention study found that both father and mother's pedometer steps predicted their child's pedometer steps on the same day, and that the mother had more effect on children's pedometer steps, especially on weekends (Holm, Wyatt, Murphy, Hill, \& Odgen, 2012). This study found that perceived parental role modeling had direct effect on children's pedometer steps, and mediated the relationship of physical activity self-efficacy with pedometer steps (Figure 6 Model F). To further examine the relationship between parental role modeling and physical activity, more reliable and valid physical activity measurement for children and parents should be used.

Although this study found that perceived parental influence partially mediated the relationships of physical activity self-efficacy and physical activity enjoyment with physical activity, more studies with larger sample size are needed to confirm this finding. 
A review of the correlates of children's physical activity concluded that: about $44 \%$ of the previous studies examined found a positive correlation between self-efficacy and physical activity, 38\% found a positive correlation between parental role modeling and physical activity, $50 \%$ found a positive correlation between parental participation with physical activity, $31 \%$ found a positive correlation between parental encouragement and physical activity, and $25 \%$ found a positive correlation between parental facilitation and physical activity (Sallis et al., 2000). Only a few studies have examined the mediators and moderators between the determinants of physical activity and physical activity (Bauman, Sallis, Dzewaltowski, \& Owen, 2002). In addition, most studies testing the YPAP model used multiple regression models to examine the relationship between the determinants of physical activity and physical activity (King et al., 2011; Welk et al., 2003) or did not find an adequately fitted model (Wattanasit, 2009). Thus, more efforts are needed to further study the potential mediators and moderators to better understand the influencing mechanisms of the determinants on physical activity by using SEM.

The role of adults rather than parents in parental influence should be also considered. During data collection, the investigator found that some grandparents picked up their grandchildren from afterschool programs; and in some families, grandparents were the primary caregivers for grandchildren. There are growing numbers of grandchildren living with their grandparents, about one in 14 U.S. children in 2010 (Scommegna, 2012). One study in 99 Hispanic children and parents found that grandparents served a protective role for childhood obesity (Pulgaron, Patino-Fernandez, Sanchez, Carrillo, \& Delamater, 2013). Evidence indicates that grandparents' BMI is positively correlated with grandchildren's BMI, but negatively correlated with 
grandchildren's physical activity levels (Polley, Spicer, Knight, \& Hartley, 2005). In addition, grandparents' physical activity levels are negatively correlated with grandchildren's TV viewing time (Polley et al., 2005). Thus, grandparental role modeling may play a significant role in promoting healthy behaviors and controlling obesity in children. However, limited consistent evidence exists to confirm the influence of grandparents on grandchildren's healthy behaviors, thus more studies are needed to examine the effects of grandparental influence on grandchildren's healthy behaviors, especially considering the growing numbers of children living with grandparents. Examining the correlates of grandparental influence with children's healthy behaviors is essential for designing an effective lifestyle program to promote physically active lifestyle and control obesity in children living with their grandparents.

Physical activity enjoyment. For children, physical activity enjoyment is a primary motivation for them to be physically active (Moore et al., 2009). In this study, physical activity enjoyment had the strongest effect on self-reported physical activity levels, but not on pedometer steps. As discussed before, children tend to overestimate their physical activity levels, thus the strongest influence of physical activity enjoyment on self-reported physical activity levels may be due to children's overestimation of their physical activity levels. Nevertheless, the most frequently reported reason for children to participate in physical activity is 'fun' (Borra, Schwartz, Spain, \& Natchipolsky, 1995). Video games that are both fun and active, requiring physical activity, especially lower body movement, have been shown to increase energy expenditure and hear rate (Biddiss \& Irwin, 2010). Therefore, future studies may consider physically active video games that are played using both upper and lower body movement as an intervention to improve 
children's self-directed physical activity levels. For intervention studies aimed at improving children's physical activity levels, fun physical activity activities and games are recommended to motivate children to participate and retain in studies. Researchers need to be aware that physical activity enjoyment may be one important factor to motivate children to start performing physical activity. However, physical activity enjoyment may be not continuous as a primary factor in maintaining children a lifelong pattern of physical activity, because enjoyment levels decrease over time especially when children lose interest in performing activities that are no longer novel. Therefore, when designing physical activity programs, children's own interests and their past physical activity experience should be considered. Building in change in programs through using a variety of activities and allowing choices among several activities at any given time is recommended for future physical activity interventions.

Physical activity self-efficacy. Physical activity self-efficacy is the most widely reported determinant of physical activity in children (Annesi et al., 2010). Some evidence indicates that an intervention that targets physical activity self-efficacy can improve children's physical activity levels (Salmon, Brown, \& Hume, 2009). This study found that physical activity self-efficacy had the second strongest effect on self-reported physical activity levels after physical activity enjoyment, and had indirect effect on pedometer steps through perceived parental influence. For self-reported physical activity levels, physical activity self-efficacy influenced children's physical activity both directly and indirectly through parental influence in this study. Although children may have had the skills and confidence to perform physical activity, parental influence is the partial mediator that helps transform thought into behavior. The positive relationship between 
physical activity self-efficacy and physical activity was supported by this study, but findings from this study emphasized the role of parental influence as a partial mediator in this relationship. Findings from a previous study indicate that physical activity selfefficacy is the partial mediator between parental influence and physical activity (Leary et al., 2013). More studies in larger samples should be conducted to further examine the mediators of the relationship between the determinants of physical activity and physical activity to provide essential information for physical activity intervention design and explanations of the effects of interventions on improving children's physical activity levels.

Environment. No significant relationship was found between perceived environment and physical activity. Environment in this study was assessed by a modified self-report 5-item scale, which may have resulted in an inaccurate estimation of children's living and school environments. Another study in 650 children found that a favorable social environment such as safety was positively correlated with children's self-reported physical activity levels, while physical environmental factors such as access to facilities was not correlated with physical activity after controlling for demographic factors (Franzini et al., 2009). This study found that only children's transportation pattern to school influenced their average pedometer steps. To better understand the relationship between environment and physical activity in elementary school children, Geographic Information System (GIS) might be used to assess children's living environment to more precisely evaluate its effect on physical activity.

Constructing supportive environments for children's physical activity is time consuming and costly, and requires the support of stakeholders such as governing bodies 
and policy makers. Especially for the physical environment, constructing a favorable physical activity environment does not guarantee that children will have access to the facilities or that they will use the facilities. However, evidence indicates that the percentages of park areas within neighborhoods increase children's physical activity levels (Roemmich et al., 2007). Although environmental change is slow and needs support from parents, teachers, and other advocates of children's health, the influence is life lasting and will benefit one generation after another.

Summary. Children's perceived parental influence has significant direct effect on both self-reported physical activity levels and pedometer steps, and partially mediates the relationships of physical activity self-efficacy and physical activity enjoyment with physical activity. Therefore, based on this study's findings, parental influence is the primary factor for targeting in future physical activity interventions in elementary school children, followed by physical activity enjoyment and then physical activity self-efficacy. In addition, perceived parental support, including parental encouragement, facilitation and involvement, seems to have more effect on self-reported physical activity levels, while perceived parental role modeling has a greater effect on average pedometer steps. The non-significant relationship between perceived environment and physical activity calls for more studies to examine this relationship using GIS to assess children's living environment.

\section{Implications for Research, Practice, and Policy}

\section{Research}

Most health behavior promotion models used in children were developed for adults (Welk, 1999). This study's findings support the hypothesis that theories that can be 
applied in adults or adolescents may not be suitable for children. Children are not independent agents, considering their financial, physical and emotional needs. Especially for young children, although they may be very confident of their ability to perform physical activity and may enjoy doing physical activity, they cannot participate in physical activity without parental support such as access to equipment and transportation. Therefore, further studies are needed to develop more effective health behavior promotion models to guide healthy lifestyle interventions among children.

In the U.S., about 15.1 million ( 1 out of 4 ) children need to take care of themselves between the time they get home from school and the time their parents get home from work (Afterschool Alliance \& JCPenny Afterschool, 2009), even though children's self-care behaviors are correlated with a high prevalence of accident, injury, risk behaviors, and poor academic performances (Vandivere, Tout, Zaslow, Calkins, \& Capizzano, 2003). Children spend about $70 \%$ of their recess time on physical activity, but only about $20 \%$ of afterschool time is spent on physical activity (Beighle, Morgan, Le Masurier, \& Pangrazi, 2006). In addition, children's sedentary activity after school increases while physical activity decreases over time (Arundell et al., 2013). Although children spend $70 \%$ of their recess time on physical activity, the physically active time from recess is only about $1 / 8$ of their total physically active time (Beighle et al., 2006). An afterschool time provides a great opportunity for children to be physically active; thus, there is a need to target children's afterschool time to increase their performance of moderate- and vigorous-intensity physical activity. This study found that children attending afterschool program were more likely to be overweight or obesity, and to 
participate in lower levels of physical activity compared to national averages. Therefore, more attention should be focused on this vulnerable population to promote their health.

In addition, this study suggests that parental influence including parental support and role modeling is the partial mediator of the relationship between physical activity self-efficacy, physical activity enjoyment, and physical activity levels in children attending afterschool programs. Future studies should target parents' influence to improve children's physical activity levels. For physical activity interventions, if only children are involved instead of both children and parents, the interventions may not have the expected effect due to the lack of mediating effect of parental influence. In addition, future studies are needed to examine the influencing mechanisms of parental influence on children's physical activity levels, especially in intervention studies.

In order to better target parental influence to improve children's physical activity and control childhood obesity, studies aimed at examining the barriers for parents to be a positive role model or supporter are needed. Future interventions to control childhood obesity should teach parents how to become a positive role model, and should identify the kinds of support parents need to provide to assist their children to be more active and healthier. Considering the significant effect of parental influence on children's physical activity levels, more reliable and valid instruments are needed. However, no comprehensive and accepted measurement instrument exists to assess parental influence on children's physical activity (Sleddens et al., 2012). To better target parental influence to improve physical activity levels and control childhood obesity, a multidimensional measurement based on theory should be developed or modified and tested from existing 
measurements to accurately assess parental influence on children's physical activity performance.

\section{Practice}

For health care providers working in clinics or schools, like pediatric medical practices, schools, community centers, and public health centers, providers should deliver health education to children's parents about the benefits of physical activity for children and the influence parents have on children's physical activity levels. Many parents know the benefit of physical activity on children's physical health, but they may not realize that physical activity also can improve children's cognitive functions such as task attention, and interference control (Chaddock-Heyman et al., 2013). By providing support and being a good role model, parents can strongly influence their children's physical activity levels.

The U.S. Preventive Services Task Force (USPSTF) suggests that health care professionals should screen for obesity among children aged 6 years and older, and refer obese children for counseling and interventions (U.S. Preventive Services Task Force, 2010). This is a grade B recommendation, which means it is highly certain that the net benefit would be moderate. Considering the high percentage of overweight and obesity in this study sample, health care providers should advocate for this recommendation, screen for childhood obesity to identify children's weight problems in a time manner, and refer overweight or obese children to an effective and fun healthy lifestyle program. Most parents and children in this study reported not knowing their children's height and weight, because children usually did not receive yearly physical assessment in clinics or schools. Thus, health care providers should work with schools to provide convenient health 
assessment to elementary school children, and provide quality health promotion interventions to children.

When designing healthy lifestyle programs to improve children's physical activity levels and control childhood obesity, health care providers should realize that to target children, they must also target children's parents, because parents are the primary influence on elementary school children's physical activity levels. Most parents agree that their child's health care providers are very helpful in keeping their child healthy, but they feel left out of the conversation during clinic appointments (Lowenstein, Perrin, Berry, et al., 2013). The relationship between parents and health care providers affects whether parents will discuss children's weight, diet and physical activity with health care providers, or will use their influence to increase children's physical activity levels. Although health care providers feel very confident in providing counseling on weight, healthy diet, and physical activity to children's parents (Lowenstein, Perrin, Campbell, et al., 2013), the 'left out' conversation between heath care providers and parents may hinder the effective delivery of information from health care providers to parents. Therefore, health care providers should work closely with children's parents to deliver quality practices to improve children's healthy behaviors and reduce childhood obesity.

When evaluating physical activity programs aimed at improving physical activity levels and controlling childhood obesity in primary care settings, the study findings on compliance rate, reliability and reactivity of wearing pedometers in elementary school children provide valuable information supporting health care providers to use pedometers to more accurately assess children's physical activity levels. Moreover, suggestions and recommendations on the measurements of obesity status in this study can help heath care 
providers to select the most reliable, valid and sensitive measures to screen for childhood obesity.

\section{Policy}

Considering the high prevalence of overweight and obesity in this study sample, effective policy strategies should be established to help screen for childhood obesity and diminish the childhood obesity epidemic in this disadvantaged population. Health care professionals should play the role of advocacy to promote childhood obesity prevention policies at the local (school and community), state and national levels. The AAP Policy Opportunities Tool was developed to summarize potential policy strategies to promote healthy, active living and eating habits, and control the childhood obesity epidemic (American Academy of Pediatrics [AAP], 2012). Health care professionals should use the AAP policy tool to guide and facilitate their advocacy job. The AAP Tool provides hospitals, schools, communities, states, and local governments a variety of policy strategies and resources to increase healthy eating habits, physical activity, BMI screening, and breastfeeding; and to decrease sedentary activity, and unhealthy beverage consumption among children. These strategies include physical education and physical activity programs, safe physical environments, farmer's markets, media campaigns, free/reduced cost lunch and afterschool meal programs, school wellness policies, and government policies at all level, which can benefit nationwide childhood obesity prevention efforts. To improve healthy behaviors and promote health in children attending afterschool programs, obesity screening policy and afterschool program policy are further discussed in this section. 
Childhood obesity screening policy. Though the USPSTF suggests health care providers screen for childhood obesity in primary care settings, only three states enacted this policy into law in 2010 (The National Conference of State Legislatures, 2010). Children spend the majority of their daily time at school, so some researchers suggest that schools should take the responsibility of screening for childhood obesity (Kubik, Fulkerson, Story, \& Rieland, 2006). Considering time and cost, BMI is usually used to assess children's obesity status, though it has been shown to be less accurate for children than for adults (Ikeda, Crawford, \& Woodward-Lopez, 2006). Although accuracy and confidentiality are concerns of childhood obesity screening, the majority of parents want to receive regular feedback about their children's weight status (Thompson \& CardHigginson, 2009). Moreover, children's parents are more aware of their children's weight status and healthy behaviors if they receive specific information on their children's BMI (Thompson \& Card-Higginson, 2009).

Though childhood obesity screening programs and legislation receive support from parents, the effect of screening programs has not been conclusively demonstrated (Soto \& White, 2010). Concerns still exist such as stigma, poor body image, and teasing related to screening children for obesity, so a protective environment is needed for the screening process. During data collection in this study, many young children who were not eligible to participate in this study wanted to get their height and weight measured. All the children in this study except two overweight/obese girls actively got their height and weight measured. The two girls were concerned that other children would see their height and weight on their copies of the pedometer logs. As a result, these two girls' heights and weights were recorded only in the investigator's pedometer logs. School staff, 
especially school nurses should be prepared to advocate for developing and implementing childhood obesity screening and reporting programs which are sensitive to children's developmental needs, to conduct and evaluate healthy lifestyle programs for high risk children, and especially to follow up with children at-risk related to their parents' responses to their children's weight problems and children's weight lost progress. To control the current childhood obesity epidemic, childhood obesity screening is needed to first identify overweight or obese children, and then to refer them to an effective lifestyle program in a timely way before they develop obesity-related health problems. To effectively advocate for legislation to require childhood obesity screening, more studies are needed to find evidence related to the effectiveness of screening programs in preventing and controlling childhood obesity, and a cost-effective and culturally sensitive measurement procedures with high specificity and sensitivity is also needed to facilitate the screening.

Afterschool program standards and policy. Afterschool programs provide an important opportunity for children to learn about health knowledge, to eat healthy afterschool snacks, and to be physically active, but no mandate for national standards or policies exist to guide practices of afterschool programs. Evidence has suggested that incorporating physical activity into afterschool programs has immediate and lasting effect on improving children's physical activity levels and controlling childhood obesity (Beets, Beighle, Erwin, \& Huberty, 2009). However, only $30 \%$ of the afterschool programs have explicit documentation on promoting physical activity opportunities in afterschool settings, and only four states outline the specific standards and guidelines, including staff training and development planning and evaluation plans, for establishing a supportive 
environment for promoting physical activity in afterschool programs (Beets, Wallner, \& Beighle, 2010). Therefore, there is a great deal of room for improvement to incorporate physical activity into afterschool programs.

Although the National AfterSchool Association developed the national afterschool standards and guidelines in 2011 in accordance with the Healthy, HungerFree Kids Act of 2010 (The White House: Office of the Press Secretary, 2010), in practice each afterschool program usually develops their own standards and guidelines instead of adopting the national guidelines due to costs and lack of training (Wiecha, Hall, Gannett, \& Roth, 2011). Evidence indicates that the majority of children attending afterschool programs, including children in this study, fail to obtain sufficient opportunities for physical activity to meet policy benchmarks (Beets, Rooney, Tilley, Beighle, \& Webster, 2010). Moreover, menus for snacks served in afterschool programs are very often high in calories, added fats, and sugars, but nearly void of fresh fruits and vegetables (Coleman, Geller, Rosenkranz, \& Dzewaltowski, 2008). Thus, even with recommendations outlining the guidelines and standards for quality afterschool programs, the practices of most afterschool programs are far from meeting standards. A recent study found that many afterschool programs adopted written policies but without specific guidelines and processes, the policies actually resulted in a decrease of physical activity and an increase of sedentary activity (Beets, Huberty, et al., 2013). However, allocating a specified percentage of time in an afterschool program's schedule to physical activity and providing attractive physical activity opportunities to children in afterschool programs strongly increases children's physical activity levels (Beets, Huberty, et al., 2013). Therefore, a written policy itself does not have any significant influence on promoting 
physical activity among children attending afterschool programs, instead, how the afterschool program organizes and implement policy matters most.

Beets, Webster and colleagues (2013) developed a conceptual framework to guide afterschool programs to translate national standards and policies into daily practices to effectively meet policy benchmarks. This framework outlines the influence of policy environment (national, state and local levels), afterschool programs staff, and organizational partnerships on children's behaviors. To improve children's physical activity and healthy eating behaviors in afterschool programs, all these partners must work together to translate guidelines and standards into daily practice schedule. More work is still needed to evaluate the application and effectiveness of this conceptual framework on promoting the quality of afterschool programs' practice.

\section{Limitations}

One limitation of this study is the cross-sectional design, which only provides information at one time point. Thus, the relationships among the determinants and physical activity are not causal relationships. Although the study findings do not prove a causal relationship, they do point out the primary effect of parental influence on children's physical activity and its role as a partial mediator in the relationships of physical activity self-efficacy and physical activity enjoyment with physical activity.

As discussed before, the seven-day physical activity scale may result in recall bias. As a result, children may overestimate their physical activity levels. Using objective measurement to assess children's physical activity levels is recommended. Moreover, the environment was assessed by a self-report 5-item scale, though the scale has good reliability and construct validity. Children may have difficulty identifying the safety of 
their living environment in the neighborhoods. Using GIS to assess children's living environment is suggested for further studies to more directly examine the relationship between environment and physical activity. Although all self-report scales used in this study have good internal consistency reliability and test-retest reliability, the construct validity of the PASES, PACES, and PAIS is questionable. Especially, the negatively worded questions influence the factorial structure of the PACES and PAIS, and are hard for elementary school children to understand. Further work is needed to evaluate the validity of these scales in a larger sample of children.

In this study, children completed the self-report surveys with the investigator reading each question, so investigator effects may exist. Children might try to answer positively to please the investigator or avoid embarrassment. Though no reactivity of wearing pedometers was found in this study, overweight or obese children did have difficulty keeping the pedometers in a vertical plane, and pedometers will not record steps if the pedometers are not in an upright position. In addition, children could and may have shaken the pedometers to increase the number of steps or make up numbers on the pedometer logs. All of these recording error and sources of bias are very hard to avoid, but this study attempted to reduce bias and error through providing easily understood guidelines and working to build trust with the children.

The study sample was recruited from ten elementary schools with afterschool programs from the Greater Clark County, IN. Generalizability of the study findings to children not involving in the afterschool programs, or to children in other locations is limited. Though the generalizability is limited, findings from this study do point out the need to conduct more research and increase attention to children attending afterschool 
programs to improve health, establish lifelong healthy behaviors, and promote the health of the large numbers of children in afterschool programs.

\section{Conclusions}

This study successfully examined the four primary determinants of physical activity among elementary school children attending afterschool programs: physical activity self-efficacy, physical activity enjoyment, parental influence and environment. All self-report scales used in this study have acceptable internal consistency reliability and test-retest reliability, although their construct validity is less than satisfactory. Using pedometers to assess children's physical activity was shown to be feasible and reliable, and no evidence of reactivity of wearing pedometers was found. Based on the findings in this study, four consecutive days, including three weekdays and one weekend day, are recommended to assess elementary school children's physical activity for future studies.

Considering the high percentage of overweight and obese children, and low levels of physical activity, children attending afterschool programs are a vulnerable population in need of more attention to improve their healthy behaviors and promote overall health. For Hispanic children who have an unemployed or part-time employed father, or a mother with a low education level, their odds to be overweight or obese are high. Overweight and obese children take fewer pedometer steps compared to non-overweight children, and children's pedometer steps are negatively related to children's BMI. Thus, improving children's physical activity levels has the potential to help control the current childhood obesity epidemic. Patterns of transportation from home to school provide an important opportunity for children to be active, but more supportive environments are necessary to facilitate children to safely walk or bicycle to school. 
Unlike the theoretical model and previous study findings, the SEM models indicate that children's perceived parental influence partially mediates the relationships of physical activity self-efficacy and physical activity enjoyment with physical activity. Perceived parental support seems to have more influence on self-reported physical activity levels, while perceived parental role model has greater influence on average pedometer steps. Including the indirect effect derived through parental influence, physical activity enjoyment has the strongest effect on self-reported physical activity levels, followed by physical activity self-efficacy. Differing from self-reported physical activity models, perceived parental influence has the strongest effect on average pedometer steps, and partially mediates the relationship between physical activity self-efficacy and average pedometer steps.

This is the first study to suggest that parental influence is an important mediator between the determinants of physical activity and the actual performance of physical activity in elementary school children attending afterschool programs. For elementary school children, parents influence children's physical activity levels by providing support through encouragement, facilitation, and involvement, and role modeling. More studies with larger sample sizes are needed to confirm this study's findings. For further health lifestyle interventions to improve children's physical activity levels and control childhood obesity, parental influence including parental support and parental role modeling is the primary targeting factor, followed by physical activity enjoyment and physical activity self-efficacy. 


\section{REFERENCES}

Adams, M. A., Johnson, W. D., \& Tudor-Locke, C. (2013). Steps/day translation of the moderate-to-vigorous physical activity guideline for children and adolescents. Int J Behav Nutr Phys Act, 10(49), 1-11. doi: 10.1186/1479-5868-10-49

Afterschool Alliance, \& JCPenny Afterschool. (2009). American after 3pm: The most indepth study of how America's children spend their afternoons. Retrieved November 02, 2013, from http://www.afterschoolalliance.org/AA3_Full_Report.pdf

Ajzen, I. (1991). The theory of planned behavior. Organizational Behavior and Human Decision Processes, 50(2), 179-211.

American Academy of Pediatrics. (2012). Preventing and treating childhood overweight and obesity: Policy tool. Retrieved December 19, 2012, from http://www2.aap.org/obesity/matrix_1.html

Anderson, S. E., Economos, C. D., \& Must, A. (2008). Active play and screen time in US children aged 4 to 11 years in relation to sociodemographic and weight status characteristics: A nationally representative cross-sectional analysis. BMC Public Health, 8(366), 1-13.

Annesi, J. J., Faigenbaum, A. D., \& Westcott, W. L. (2010). Relations of transtheoretical model stage, self-efficacy, and voluntary physical activity in African American preadolescents. Research Quarterly for Exercise and Sport, 81(2), 239-244. 
Arundell, L., Ridgers, N. D., Veitch, J., Salmon, J., Hinkley, T., \& Timperio, A. (2013). 5-year changes in afterschool physical activity and sedentary behavior. Am J Prev Med, 44(6), 605-611. doi: 10.1016/j.amepre.2013.01.029

Ball, G. D. C., Marshall, J. D., \& McCargar, L. J. (2003). Fatness and fitness in obese children at low and high health risk. Pediatric Exercise Science, 15(4), 392-405.

Bandura, A. (1977). Self-Efficacy - Toward a unifying theory of behavioral change. Psychological Review, 84(2), 191-215.

Bandura, A. (1986). Social foundation for thought and action: A social cognitive theory. Englewood Cliffs, NJ: Prentice Hall.

Bandura, A. (1997). Self-efficacy: The exercise of control. New York: W. H. Freeman and Company.

Baranowski, T., Cullen, K. W., Nicklas, T., Thompson, D., \& Baranowski, J. (2003). Are current health behavioral change models helpful in guiding prevention of weight gain efforts? Obesity Research, 11(Suppl), 23S-43S. doi: 10.1038/oby.2003.222

Baranowski, T., Dworkin, R. J., Cieslik, C. J., Hooks, P., Clearman, D. R., Ray, L., . . Nader, P. R. (1984). Reliability and validity of self report of aerobic activity Family Health Project. Research Quarterly for Exercise and Sport, 55(4), 309317.

Bartholomew, J. B., Jowers, E. M., Loukas, A., \& Allua, S. (2005). Validation of the selfefficacy and beliefs scale for physical activity. Medicine and Science in Sports and Exercise, 37, S252-S253.

Bauman, A. E., Sallis, J. F., Dzewaltowski, D. A., \& Owen, N. (2002). Toward a better understanding of the influences on physical activity: The role of determinants, 
correlates, causal variables, mediators, moderators, and confounders. Am J Prev Med, 23(2 Suppl), 5-14.

Beets, M. W., Beighle, A., Erwin, H. E., \& Huberty, J. L. (2009). After-school program impact on physical activity and fitness: A meta-analysis. Am J Prev Med, 36(6), 527-537. doi: 10.1016/j.amepre.2009.01.033

Beets, M. W., Cardinal, B. J., \& Alderman, B. L. (2010). Parental social support and the physical activity-related behaviors of youth: A review. Health Education \& Behavior, 37(5), 621-644. doi: 10.1177/1090198110363884

Beets, M. W., Huberty, J., Beighle, A., Moore, J. B., Webster, C., Ajja, R., \& Weaver, G. (2013). Impact of policy environment characteristics on physical activity and sedentary behaviors of children attending afterschool programs. Health Educ Behav, 40(3), 296-304. doi: 10.1177/1090198112459051

Beets, M. W., Rooney, L., Tilley, F., Beighle, A., \& Webster, C. (2010). Evaluation of policies to promote physical activity in afterschool programs: Are we meeting current benchmarks? Prev Med, 51(3-4), 299-301. doi:

10.1016/j.ypmed.2010.07.006

Beets, M. W., Wallner, M., \& Beighle, A. (2010). Defining standards and policies for promoting physical activity in afterschool programs. $J$ Sch Health, 80(8), 411-417. doi: $10.1111 / j .1746-1561.2010 .00521 . x$

Beets, M. W., Webster, C., Saunders, R., Huberty, J. L., \& Healthy Afterschool Program Network. (2013). Translating policies into practice: A framework to prevent childhood obesity in afterschool programs. Health Promot Pract, 14(2), 228-237. doi: $10.1177 / 1524839912446320$ 
Beighle, A., Alderman, B., Morgan, C. F., \& Le Masurier, G. (2008). Seasonality in children's pedometer-measured physical activity levels. Research Quarterly for Exercise and Sport, 79(2), 256-260.

Beighle, A., Morgan, C. F., Le Masurier, G., \& Pangrazi, R. P. (2006). Children's physical activity during recess and outside of school. J Sch Health, 76(10), 516520. doi: 10.1111/j.1746-1561.2006.00151.x

Biddiss, E., \& Irwin, J. (2010). Active video games to promote physical activity in children and youth: a systematic review. Arch Pediatr Adolesc Med, 164(7), 664672. doi: 10.1001/archpediatrics.2010.104

Bock, R., \& Miller, M. G. (2008). Children's physical activity drops from age 9 to 15 , NIH study indicates. Retrieved August 15, 2012, from http://www.nih.gov/news/health/jul2008/nichd-15.htm

Bois, J. E., Sarrazin, P. G., Brustad, R. J., Trouilloud, D. O., \& Cury, F. (2005). Elementary schoolchildren's perceived competence and physical activity involvement: The influence of parents' role modelling behaviours and perceptions of their child's competence. Psychology of Sport and Exercise, 6(4), 381-397. doi: 10.1016/j.psychsport.2004.03.003

Borra, S. T., Schwartz, N. E., Spain, C. G., \& Natchipolsky, M. M. (1995). Food, physical activity, and fun: Inspiring America's kids to more healthful lifestyles. $J$ Am Diet Assoc, 95(7), 816-823.

Boufous, S., Finch, C., \& Bauman, A. (2004). Parental safety concerns - A barrier to sport and physical activity in children? Australian and New Zealand Journal of Public Health, 28(5), 482-486. 
Brown, H., \& Prescott, R. (2006). Applied mixed models in Medicine. The Atrium, Southern Gate, Chichester, West Sussex: John Wiley \& Sons, Ltd.

Brown, T., \& Summerbell, C. (2009). Systematic review of school-based interventions that focus on changing dietary intake and physical activity levels to prevent childhood obesity: An update to the obesity guidance produced by the National Institute for Health and Clinical Excellence. Obesity Reviews, 10(1), 110-141. doi: 10.1111/j.1467-789X.2008.00515.x

Brustad, R. J. (1993). Who will go out and play - Parental and psychological influences on childrens attraction to physical-activity. Pediatric Exercise Science, 5(3), 210223.

Bukara-Radujkovic, G., \& Zdravkovic, D. (2009). Physical activity as an important determinant in developing childhood obesity. Med Pregl, 62(3-4), 107-113.

Byrne, B. M. (2010). Structural equation modeling with AMOS: Basic concepts, applications, and programming. New York: Taylor and Francis group, LLC.

Caspersen, C. J., Powell, K. E., \& Christenson, G. M. (1985). Physical activity, exercise, and physical fitness: Definitions and distinctions for health-related research. Public Health Reports, 100(2), 126-131.

Centers for Disease Control and Prevention. (2005). Barriers to children walking to or from school: United States, 2004. MMWR, 54(38), 949-952.

Centers for Disease Control and Prevention. (2011a). About BMI for children and teens. Retrieved July 15, 2012, from http://www.cdc.gov/healthyweight/assessing/bmi/childrens_bmi/about_childrens_ bmi.html 
Centers for Disease Control and Prevention. (2011b). Children (Approximate Ages 4-11). Retrieved November 13, 2012, from http://www.cdc.gov/parents/children/index.html

Centers for Disease Control and Prevention. (2011c). How much physical activity do children need? Retrieved November 13, 2012, from http://www.cdc.gov/physicalactivity/everyone/guidelines/children.html Centers for Disease Control and Prevention. (2011d). Measuring children's height and weight accurately at home. Retrieved November 13, 2012, from http://www.cdc.gov/healthyweight/assessing/bmi/childrens_bmi/measuring_childr en.html

Centers for Disease Control and Prevention. (2011e). Overweight and obesity. Retrieved November 13, 2012, from http://www.cdc.gov/obesity/childhood/basics.html Chaddock-Heyman, L., Erickson, K. I., Voss, M. W., Knecht, A. M., Pontifex, M. B., Castelli, D. M., ... Kramer, A. F. (2013). The effects of physical activity on functional MRI activation associated with cognitive control in children: A randomized controlled intervention. Front Hum Neurosci, 7(72), 1-13. doi: 10.3389 /fnhum.2013.00072

Cheung-Peggy, P.Y., \& Chow, B. C. (2010). Parental mediatory role in children's physical activity participation. Health Education, 110(5), 351-366.

Claudio, L., \& Stingone, J. A. (2008). Improving sampling and response rates in children's health research through participatory methods. Journal of School Health, 78(8), 445-451. doi: 10.1111/j.1746-1561.2008.00328.x 
Clemes, S. A., \& Biddle, S. J. H. (2013). The use of pedometers for monitoring physical activity in children and adolescents: Measurement considerations. Journal of Physical Activity \& Health, 10(2), 249-262.

Cliff, D. P., \& Janssen, X. (2011). Levels of habitual physical activity in early childhood. Retrieved March 08, 2013, from http://www.childencyclopedia.com/documents/Cliff-JanssenANGxp1.pdf

Coleman, K. J., Geller, K. S., Rosenkranz, R. R., \& Dzewaltowski, D. A. (2008). Physical activity and healthy eating in the after-school environment. Journal of School Health, 78(12), 633-640.

Committee on Environmental Health. (2009). The built environment: Designing communities to promote physical activity in children. Pediatrics, 123(6), 15911598. doi: 10.1542/peds.2009-0750

Communities In Schools of Clark Co. (2010). Communities In Schools of Clark County Retrieved January 24, 2012, from http://www.cisofclark.org/index.php?m1=22\&id=1

Cordery, J. L., \& Sevastos, P. P. (1993). Responses to the original and revised job diagnostic survey - Is education a factor in responses to negatively worded items. Journal of Applied Psychology, 78(1), 141-143. doi: 10.1037//00219010.78 .1 .141

Cortina, J. M. (1993). What is coefficient alpha - An examination of theory and applications. Journal of Applied Psychology, 78(1), 98-104. doi: 10.1037//00219010.78 .1 .98 
Council on Sports Medicine and Fitness, \& Council on School Health. (2006). Active healthy living: Prevention of childhood obesity through increased physical activity. Pediatrics, 117(5), 1834-1842.

Cox, C. L., Martin-Ginis, K. A., \& Petruzzello, S. J. (2006). The psychology of exercise: Intergrating theory and practice. Scottsdale, Arizona: Holcomb-Hathaway.

Craig, C. L., Cameron, C., \& Tudor-Locke, C. (2013). Relationship between parent and child pedometer-determined physical activity: A sub-study of the CANPLAY surveillance study. International Journal of Behavioral Nutrition and Physical Activity, 10(8), 1-8.

Craig, C. L., Tudor-Locke, C., Cragg, S., \& Cameron, C. (2010). Process and treatment of pedometer data collection for youth: The canadian physical activity levels among youth study. Medicine and Science in Sports and Exercise, 42(3), 430-435. doi: 10.1249/Mss.0b013e3181b67544

Crespo, C. J., Smit, E., Troiano, R. P., Bartlett, S. J., Macera, C. A., \& Andersen, R. E. (2001). Television watching, energy intake, and obesity in US children: Results from the third National Health and Nutrition Examination Survey, 1988-1994. Archives of Pediatrics \& Adolescent Medicine, 155(3), 360-365.

Crocker, P. R., Bailey, D. A., Faulkner, R. A., Kowalski, K. C., \& McGrath, R. (1997). Measuring general levels of physical activity: Preliminary evidence for the physical activity questionnaire for older children. Medicine and Science in Sports and Exercise, 29(10), 1344-1349. 
Crocker, P. R., Bouffard, M., \& Gessaroli, M. E. (1995). Measuring enjoyment in youth sport settings: A confirmatory factor analysis of the physical activity enjoyment scale. Journal of Sport \& Exercise Psychology, 17(2), 200-205.

Davis, M. M., Gance-Cleveland, B., Hassink, S., Johnson, R., Paradis, G., \& Resnicow, K. (2007). Recommendations for prevention of childhood obesity. Pediatrics, 120, S229-S253. doi: 10.1542/peds.2007-2329E

Davison, K. K., Downs, D. S., \& Birch, L. L. (2006). Pathways linking perceived athletic competence and parental support at age 9 years to girls' physical activity at age 11 years. Research Quarterly for Exercise and Sport, 77(1), 23-31.

Davison, K. K., \& Lawson, C. T. (2006). Do attributes in the physical environment influence children's physical activity? A review of the literature. International Journal of Behavioral Nutrition and Physical Activity, 3(19), 1-17. doi:10.1186/1479-5868-3-19

de Onis, M., Onyango, A. W., Borghi, E., Siyam, A., Nishida, C., \& Siekmann, J. (2007). Development of a WHO growth reference for school-aged children and adolescents. Bull World Health Organ, 85(9), 660-667.

Dehghan, M., \& Merchant, A. T. (2008). Is bioelectrical impedance accurate for use in large epidemiological studies? Nutrition Journal, 7(26), 1-7. doi: 10.1186/1475$2891-7-26$

DeVellis, R. F. (2003). Scale development: Theory and application. California: Sage Publication, Inc. 
DiLorenzo, T. M., Stucky-Ropp, R. C., Vander Wal, J. S., \& Gotham, H. J. (1998).

Determinants of exercise among children: A longitudinal analysis. Preventive Medicine, 27(3), 470-477.

Dishman, R. K., Motl, R. W., Saunders, R., Felton, G., Ward, D. S., Dowda, M., \& Pate, R. R. (2004). Self-efficacy partially mediates the effect of a school-based physical-activity intervention among adolescent girls. Preventive Medicine, 38(5), 628-636. doi: 10.1016/j.ypmed.2003.12.007

Dishman, R. K., Motl, R. W., Saunders, R., Felton, G., Ward, D. S., Dowda, M., \& Pate, R. R. (2005). Enjoyment mediates effects of a school-based physical-activity intervention. Medicine and Science in Sports and Exercise, 37(3), 478-487. doi: 10.1249/01.Mss.0000155391.62733.A7

Dishman, R. K., Motl, R. W., Saunders, R. P., Dowda, M., Felton, G., Ward, D. S., \& Pate, R. R. (2002a). Examining social-cognitive determinants of intention and physical activity among black and white adolescent girls using structural equation modeling. Health Psychology, 21(5), 459-467. doi: 10.1037//0278-6133.21.5.459

Dishman, R. K., Motl, R. W., Saunders, R. P., Dowda, M., Felton, G., Ward, D. S., \& Pate, R. R. (2002b). Factorial invariance and latent mean structure of questionnaires measuring social-cognitive determinants of physical activity among black and white adolescent girls. Preventive Medicine, 34(1), 100-108. doi: 10.1006/pmed.2001.0959

Dobbins, M., De Corby, K., Robeson, P., Husson, H., \& Tirilis, D. (2009). School-based physical activity programs for promoting physical activity and fitness in children 
and adolescents aged 6-18. Cochrane Database of Systematic Reviews, (1), CD007651. doi: 10.1002/14651858.CD007651

Donnelly, J. E., Greene, J. L., Gibson, C. A., Smith, B. K., Washburn, R. A., Sullivan, D. K., . . W Williams, S. L. (2009). Physical Activity Across the Curriculum (PAAC): A randomized controlled trial to promote physical activity and diminish overweight and obesity in elementary school children. Preventive Medicine, 49(4), 336-341. doi: 10.1016/j.ypmed.2009.07.022

DuCharme, K. A., \& Brawley, L. R. (1995). Predicting the intentions and behavior of exercise initiates using two forms of self-efficacy. Journal of Behavioral Medicine, 18(5), 479-497.

Duke, J., \& Huhman, M. (2003). Physical activity levels among children aged 9-13 years - United States, 2002. MMWR, 52(33), 785-788.

Duncan, J. S., Schofield, G., \& Duncan, E. K. (2006). Pedometer-determined physical activity and body composition in New Zealand children. Medicine and Science in Sports and Exercise, 38(8), 1402-1409.

Dzewaltowski, D. A., Geller, K. S., Rosenkranz, R. R., \& Karteroliotis, K. (2010). Children's self-efficacy and proxy efficacy for after-school physical activity. Psychology of Sport and Exercise, 11(2), 100-106. doi:

10.1016/j.psychsport.2009.08.001

Egan, L. C., Santos, L. R., \& Bloom, P. (2007). The origins of cognitive dissonance: evidence from children and monkeys. Psychol Sci, 18(11), 978-983. doi: 10.1111/j.1467-9280.2007.02012.x 
Eisenmann, J. C., Laurson, K. R., Wickel, E. E., Gentile, D., \& Walsh, D. (2007). Utility of pedometer step recommendations for predicting overweight in children. International Journal of Obesity, 31(7), 1179-1182. doi: 10.1038/sj.ijo.0803553

Elder, J. P., Lytle, L., Sallis, J. F., Young, D. R., Steckler, A., Simons-Morton, D., . . . Ribisl, K. (2007). A description of the social-ecological framework used in the trial of activity for adolescent girls (TAAG). Health Education Research, 22(2), 155-165. doi: 10.1093/her/cyl059

Engeland, A., Bjorge, T., Sogaard, A. J., \& Tverdal, A. (2003). Body mass index in adolescence in relation to total mortality: 32-year follow-up of 227,000 Norwegian boys and girls. American Journal of Epidemiology, 157(6), 517-523.

Evans, C. A., Jordan, A. B., \& Horner, J. (2011). Only two hours? A qualitative study of the challenges parents perceive in restricting child television time. Journal of Family Issues, 32(9), 1223-1244. doi: 10.1177/0192513x11400558

Fakhouri, T. H. I., Hughes, J. P., Brody, D. J., Kit, B. K., \& Ogden, C. L. (2013). Physical activity and screen-time viewing among elementary school-aged children in the United States from 2009 to 2010. JAMA Pediatrics, 167(3), 223-229.

Festinger, L. (1962). A theory of cognitive dissonance. Stanford, CA: Stanford University Press.

Flores, G., \& Lin, H. (2013). Trends in racial/ethnic disparities in medical and oral health, access to care, and use of services in US children: Has anything changed over the years? International Journal for Equity in Health, 12(10), 1-16.

Franzini, L., Elliott, M. N., Cuccaro, P., Schuster, M., Gilliland, M. J., Grunbaum, J. A., ... Tortolero, S. R. (2009). Influences of physical and social neighborhood 
environments on children's physical activity and obesity. American Journal of Public Health, 99(2), 271-278.

Freedson, P. S., \& Miller, K. (2000). Objective monitoring of physical activity using motion sensors and heart rate. Research Quarterly for Exercise and Sport, 71(2 Suppl), S21-29.

Fried, Y., \& Ferris, G. R. (1986). The dimensionality of job characteristics: Some neglected issues. Journal of Applied Psychology, 71(3), 419-426. doi: 10.1037/0021-9010.71.3.419

Fu, W. P., Lee, H. C., Ng, C. J., Tay, Y. K., Kau, C. Y., Seow, C. J., . . Hong, C. Y. (2003). Screening for childhood obesity: International vs population-specific definitions. Which is more appropriate? International Journal of Obesity and Related Metabolic Disorders, 27(9), 1121-1126. doi: 10.1038/sj.ijo.0802385

Gao, S. Y., Mokhtarian, P. L., \& Johnston, R. A. (2008). Nonnormality of data in structural equation models. Transportation Research Record, (2082), 116-124. doi: $10.3141 / 2082-14$

Glanz, K., Rimer, B. K., \& Viswanath, K. (2008). Health behavior and health education. San Francisco, CA: John Wiley \& Sons, Inc.

Godin, G., \& Conner, M. (2008). Intention-behavior relationship based on epidemiologic indices: An application to physical activity. American Journal of Health Promotion, 22(3), 180-182. doi: 10.4278/ajhp.22.3.180

Goran, M. I., \& Treuth, M. S. (2001). Energy expenditure, physical activity, and obesity in children. Pediatric clinics of North America, 48(4), 931-953. 
Green, L. W., \& Kreuter, M. W. (2005). Health program planning: An educational and ecological approach (4th ed.). New York: McGraw-Hill Companies, Inc.

Green, L. W., Richard, L., \& Potvin, L. (1996). Ecological foundations of health promotion. American Journal of Health Promotion, 10(4), 270-281.

Guthold, R., Cowan, M. J., Autenrieth, C. S., Kann, L., \& Riley, L. M. (2010). Physical activity and sedentary behavior among schoolchildren: A 34-country comparison. Journal of Pediatrics, 157(1), 43-49.e1. doi: 10.1016/j.jpeds.2010.01.019

Handy, S. L., Boarnet, M. G., Ewing, R., \& Killingsworth, R. E. (2002). How the built environment affects physical activity: Views from urban planning. American Journal of Preventive Medicine, 23(2 Suppl), 64-73.

Harris, K. C., Kuramoto, L. K., Schulzer, M., \& Retallack, J. E. (2009). Effect of schoolbased physical activity interventions on body mass index in children: A metaanalysis. CMAJ: Canadian Medical Association Journal, 180(7), 719-726.

Heitzler, C. D., Martin, S. L., Duke, J., \& Huhman, M. (2006). Correlates of physical activity in a national sample of children aged 9-13 years. Preventive Medicine, 42(4), 254-260.

Hesketh, K., Crawford, D., \& Salmon, J. (2006). Children's television viewing and objectively measured physical activity: Associations with family circumstance. International Journal of Behavioral Nutrition and Physical Activity, 3(36), 1-10. doi: $10.1186 / 1479-5868-3-36$

Hills, A. P., Andersen, L. B., \& Byrne, N. M. (2011). Physical activity and obesity in children. British Journal of Sports Medicine, 45(11), 866-870. doi: 10.1136/bjsports-2011-090199 
Hills, A. P., King, N. A., \& Armstrong, T. P. (2007). The contribution of physical activity and sedentary behaviours to the growth and development of children and adolescents: Implications for overweight and obesity. Sports Medicine, 37(6), 533-545.

Hills, A. P., Okely, A. D., \& Baur, L. A. (2010). Addressing childhood obesity through increased physical activity. Nature Reviews Endocrinology, 6(10), 543-549. doi: 10.1038/nrendo.2010.133

Hoelter, J. W. (1983). The analysis of covariance-structures - Goodness-of-fit indexes. Sociological Methods \& Research, 11(3), 325-344. doi: $10.1177 / 0049124183011003003$

Holm, K., Wyatt, H., Murphy, J., Hill, J., \& Odgen, L. (2012). Parental influence on child change in physical activity during a family-based intervention for child weight gain prevention. J Phys Act Health, 9(5), 661-669.

Howley, E. T. (2001). Type of activity: Resistance, aerobic and leisure versus occupational physical activity. Medicine and Science in Sports and Exercise, 33(6 Suppl), S364-369; discussion S419-320.

Hu, L. T., \& Bentler, P. M. (1999). Cutoff criteria for fit indexes in covariance structure analysis: Conventional criteria versus new alternatives. Structural Equation Modeling, 6(1), 1-55. doi: 10.1080/10705519909540118

Huhman, M. E., Potter, L. D., Duke, J. C., Judkins, D. R., Heitzler, C. D., \& Wong, F. L. (2007). Evaluation of a national physical activity intervention for children: VERB campaign, 2002-2004. American Journal of Preventive Medicine, 32(1), 38-43. 
Humbert, M. L., Chad, K. E., Spink, K. S., Muhajarine, N., Anderson, K. D., Bruner, M. W., .. . Gryba, C. R. (2006). Factors that influence physical activity participation among high- and low-SES youth. Qualitative Health Research, 16(4), 467-483. doi: $10.1177 / 1049732305286051$

Ikeda, J. P., Crawford, P. B., \& Woodward-Lopez, G. (2006). BMI screening in schools: Helpful or harmful. Health Educ Res, 21(6), 761-769. doi: 10.1093/her/cyl144

Janssen, I., Katzmarzyk, P. T., Boyce, W. F., Vereecken, C., Mulvihill, C., Roberts, C., ... Health Behavior School-Aged Children Obesity Working Group. (2005). Comparison of overweight and obesity prevalence in school-aged youth from 34 countries and their relationships with physical activity and dietary patterns. Obesity Reviews, 6(2), 123-132.

Jordan, A. B., \& Robinson, T. N. (2008). Children, television viewing, and weight status: Summary and recommendations from an expert panel meeting. Annals of the American Academy of Political and Social Science, 615, 119-132. doi: $10.1177 / 0002716207308681$

Kaiser Family Foundation. (2011a). Percent of children (10-17) who are overweight or obese. Retrieved October 29, 2013, from http://kff.org/other/stateindicator/overweightobese-children/

Kaiser Family Foundation. (2011b). United States: Population distribution of children by race/ethnicity, states (2010-2011), U.S. Retrieved February 6, 2013, from http://www.statehealthfacts.org/profileind.jsp?rgn=1\&ind=7

Kaiser, H. F. (1974). An index of factorial simplicity. Psychometrika, 39(1), 31-36. 
Katherine, C. (2009). Relationship between parental role-modeling of physical activity and child's physical activity. Retrieved December 27, 2012, from https://digarchive.library.vcu.edu/handle/10156/2420

Kendzierski, D., \& Decarlo, K. J. (1991). Physical-activity enjoyment scale - 2 validation studies. Journal of Sport \& Exercise Psychology, 13(1), 50-64.

King, K. M. (2008). Predisposing, reinforcing, and enabling predictors of middle school children's after-school physical activity participation. (Doctor Dissertation, Southern Illinois University Carbondale). Retrieved November 22, 2013 from http://opensiuc.lib.siu.edu/dissertations/261/

King, K. M., Ogletree, R. J., Fetro, J. V., Brown, S. L., \& Partridge, J. A. (2011). Predisposing, reinforcing and enabling predictors of middle school children's after-school physical activity participation. American Journal of Health Education, 42(3), 142-153.

Kipping, R. R., Jago, R., \& Lawlor, D. A. (2008). Obesity in children. Part 1: Epidemiology, measurement, risk factors, and screening. BMJ, 337, a1824. doi: 10.1136/bmj.a1824

Kohl, H. W., Fulton, J. E., \& Caspersen, C. J. (2000). Assessment of physical activity among children and adolescents: A review and synthesis. Preventive Medicine, 31(2), S54-S76. doi: 10.1006/pmed.1999.0542

Kowalski, K. C., Crocker, P. R., \& Donen, R. M. (2004). The Physical Activity Questionnaire for Older Children (PAQ-C) and Adolescents (PAQ-A) manual. Saskatoon, CA: University of Saskatchewan. 
Kowalski, K. C., Crocker, P. R., \& Faulkner, R. A. (1997). Validation of the physical activity questionnaire for older children. Pediatric Exercise Science, 9(2), 174186.

Kroll, T., Kehn, M., Ho, P. S., \& Groah, S. (2007). The SCI exercise self-efficacy scale (ESES): Development and psychometric properties. International Journal of Behavioral Nutrition and Physical Activity, 4(34), 1-6. doi: 10.1186/1479-5868-434

Kubik, M. Y., Fulkerson, J. A., Story, M., \& Rieland, G. (2006). Parents of elementary school students weigh in on height, weight, and body mass index screening at school. J Sch Health, 76(10), 496-501. doi: 10.1111/j.1746-1561.2006.00147.x

Langille, J. L. D., \& Rodgers, W. M. (2010). Exploring the influence of a social ecological model on school-based physical activity. Health Education \& Behavior, 37(6), 879-894. doi: 10.1177/1090198110367877

Leary, J. M., Lilly, C. L., Dino, G., Loprinzi, P. D., \& Cottrell, L. (2013). Parental influences on 7-9 year olds' physical activity: A conceptual model. Prev Med, 56(5), 341-344. doi: 10.1016/j.ypmed.2013.02.005

Lee, S. M., Burgeson, C. R., Fulton, J. E., \& Spain, C. G. (2007). Physical education and physical activity: Results from the school health policies and programs study 2006. Journal of School Health, 77(8), 435-463.

Ling, J. (2012). Psychosocial determinants of physical activity among elementary school children: A feasibility study. Unpublished raw data. 
Ling, J., King, K., Speck, B., Kim, S., \& Wu, D. (2013 in press). Preliminary assessment of a school-based healthy lifestyle intervention among rural children. Journal of School Health.

Lobstein, T., Baur, L., Uauy, R., \& IASO International Obesity TaskForce. (2004). Obesity in children and young people: A crisis in public health. Obesity Reviews, 5(Suppl. 1), 4-85.

Lowenstein, L. M., Perrin, E. M., Berry, D., Vu, M. B., Pullen D. L., Cai, J., . . . Ammerman, A. S. (2013). Childhood obesity prevention: Fathers' reflections with healthcare providers. Child Obes, 9(2), 137-143. doi: 10.1089/chi.2012.0111

Lowenstein, L. M., Perrin, E. M., Campbell, M. K., Tate, D. F., Cai, J., \& Ammerman, A. S. (2013). Primary care providers' self-efficacy and outcome expectations for childhood obesity counseling. Child Obes, 9(3), 208-215. doi: 10.1089/chi.2012.0119

Lox, C. L., Martin-Ginis, K. A., \& Petruzzello, S. J. (2006). The psychology of exercise. Scottsdale: Holcomb Hathaway, Publishers, Inc.

Luszczynska, A., Schwarzer, R., Lippke, S., \& Mazurkiewicz, M. (2011). Self-efficacy as a moderator of the planning-behaviour relationship in interventions designed to promote physical activity. Psychology and Health, 26(2), 151-166. doi: $10.1080 / 08870446.2011 .531571$

MacKelvie, K. J., Petit, M. A., Khan, K. M., Beck, T. J., \& McKay, H. A. (2004). Bone mass and structure are enhanced following a 2-year randomized controlled trial of exercise in prepubertal boys. Bone, 34(4), 755-764. doi:

10.1016/j.bone.2003.12.017 
Marsh, H. W. (1984). The bias of negatively worded items in ratings scales for preadolescent children: A cognitive-developmental phenomenon. Washington, D.C.: Educational Resources Information Center.

Marshall, S. J., Gorely, T., \& Biddle, S. J. H. (2006). A descriptive epidemiology of screen-based media use in youth: A review and critique. Journal of Adolescence, 29(3), 333-349. doi: 10.1016/j.adolescence.2005.08.016

Martin, J. J., Oliver, K., \& McCaughtry, N. (2007). The theory of planned behavior: Predicting physical activity in Mexican American children. Journal of Sport \& Exercise Psychology, 29(2), 225-238.

Martinson, B. C., Lazovich, D., Lando, H. A., Perry, C. L., McGovern, P. G., \& Boyle, R. G. (2000). Effectiveness of monetary incentives for recruiting adolescents to an intervention trial to reduce smoking. Preventive Medicine, 31(6), 706-713. doi: 10.1006/pmed.2000.0762

McClain, J. J., \& Tudor-Locke, C. (2009). Objective monitoring of physical activity in children: considerations for instrument selection. Journal of Science and Medicine in Sport/Sports Medicine Australia, 12(5), 526-533. doi:

10.1016/j.jsams.2008.09.012

McGraw, K. O., \& Wong, S. P. (1996). Forming inferences about some intraclass correlation coefficients. Psychological Methods, 1(1), 30-46. doi: 10.1037/1082$989 x \cdot 1.4 .390$

McLeroy, K. R., Bibeau, D., Steckler, A., \& Glanz, K. (1988). An ecological perspective on health promotion programs. Health Education Quarterly, 15, 351-377. 
McNamara, E., Hudson, Z., \& Taylor, S. J. (2010). Measuring activity levels of young people: The validity of pedometers. British Medical Bulletin, 95, 121-137. doi: $10.1093 / \mathrm{bmb} / \mathrm{ldq} 016$

McVeigh, J. A., Norris, S. A., \& de Wet, T. (2004). The relationship between socioeconomic status and physical activity patterns in South African children. Acta Paediatrica, 93(7), 982-988. doi: 10.1080/08035250410024961

Mitre, N., Lanningham-Foster, L., Foster, R., \& Levine, J. A. (2009). Pedometer accuracy for children: Can we recommend them for our obese population? Pediatrics, 123(1), e127-e131.

Molnar, B. E., Gortmaker, S. L., Bull, F. C., \& Buka, S. L. (2004). Unsafe to play? Neighborhood disorder and lack of safety predict reduced physical activity among urban children and adolescents. American Journal of Health Promotion, 18(5), 378-386.

Moore, J. B., Yin, Z., Hanes, J., Duda, J., Gutin, B., \& Barbeau, P. (2009). Measuring enjoyment of physical activity in children: Validation of the physical activity enjoyment scale. Journal of Applied Sport Psychology, 21, S116-S129. doi: $10.1080 / 10413200802593612$

Motl, R. W. (2007). Theoretical models for understanding physical activity behavior among children and adolescents-social cognitive theory and self-determination theory. Journal of Teaching in Physical Education, 26(4), 350-357.

Motl, R. W., Dishman, R. K., Saunders, R., Dowda, M., Felton, G., \& Pate, R. R. (2001). Measuring enjoyment of physical activity in adolescent girls. American Journal of Preventive Medicine, 21(2), 110-117. 
Motl, R. W., Dishman, R. K., Ward, D. S., Saunders, R. P., Dowda, M., Felton, G., \& Pate, R. R. (2005). Perceived physical environment and physical activity across one year among adolescent girls: Self-efficacy as a possible mediator? Journal of Adolescent Health, 37(5), 403-408. doi: 10.1016/j.jadohealth.2004.10.004

Moyer, V. A., Klein, J. D., Ockene, J. K., Teutsch, S. M., Johnson, M. S., \& Allan, J. D. (2005). Screening for overweight in children and adolescents: Where is the evidence? A commentary by the childhood obesity working group of the US Preventive Services Task Force. Pediatrics, 116(1), 235-238. doi:

10.1542/peds.2005-0305

Mundfrom, D. J., Shaw, D. G., \& Ke, T. L. (2005). Minimum sample size recommendations for conducting factor analyses. International Journal of Testing, 5(2), 159-168.

National Association for Sport and Physical Education. (2010). Shape of the nation report. Retrieved December 19, 2012, from http://www.aahperd.org/naspe/publications/upload/Shape-of-the-Nation-2010Final.pdf

National Association for Sport and Physical Education. (2012). Comprehensive school physical activity programs package. Retrieved December 21, 2012, from http://www.aahperd.org/naspe/publications/teachingTools/cspa.cfm

National Association for Sport and Physical Education. (2013). Is it physical education or physical activity? Retrieved February 18, 2013, from http://www.aahperd.org/naspe/publications/teachingTools/PAvsPE.CFM 
National Center for Educational Statistics. (2012). Search for public schools. Retrieved January 24, 2013, from http://nces.ed.gov/ccd/schoolsearch/

National Weather Service Weather Forecast Office. (2013). Indianapolis climatological information: Indianapolis monthly summaries 1996-2013. Retrieved November 26, 2013, from http://www.crh.noaa.gov/ind/?n=localcli

Nowicka, P., \& Flodmark, C. E. (2007). Physical activity - Key issues in treatment of childhood obesity. Acta Paediatrica Supplement, 96(454), 39-45. doi: 10.1111/j.1651-2227.2007.00169.x

Nunnally, J. C., \& Bernstein, I. H. (1994). Psychometric theory (3rd ed.). New York: McGraw-Hill, Inc.

Ogden, C. (2010). Prevalence of obesity among children and adolescents: United States, trends 1963-1965 through 2007-2008. Retrieved August 15, 2012, from http://www.cdc.gov/nchs/data/hestat/obesity_child_07_08/obesity_child_07_08.ht $\mathrm{m}$

Olshansky, S. J., Passaro, D. J., Hershow, R. C., Layden, J., Carnes, B. A., Brody, J., . . . Ludwig, D. S. (2005). A potential decline in life expectancy in the United States in the 21st century. New England Journal of Medicine, 352(11), 1138-1145.

Phan, T., \& Glander, M. (2007). Documentation to the NCES common core of data local education agency locale code file: School year 2005-06 (NCES 2007-388).

Washington, DC: National Center for Education Statistics, Institute of Education Sciences, U.S. Department of Education. 
Phillips, S. M., \& Shulman, R. J. (2012). Measurement of body composition in children. Retrieved February 19, 2013, from http://www.uptodate.com/contents/measurement-of-body-composition-in-children Piaget, J. (1952). The origins of intelligence in children. New York: International University Press.

Polit, D. F., \& Beck, C. T. (2004). Nursing research: Principles and methods. Philadelphia, PA: Lippincott Williams \& Wilkins.

Polley, D. C., Spicer, M. T., Knight, A. P., \& Hartley, B. L. (2005). Intrafamilial correlates of overweight and obesity in African-American and Native-American grandparents, parents, and children in rural Oklahoma. J Am Diet Assoc, 105(2), 262-265. doi: 10.1016/j.jada.2004.11.004

Prentice-Dunn, H., \& Prentice-Dunn, S. (2012). Physical activity, sedentary behavior, and childhood obesity: A review of cross-sectional studies. Psychol Health Med, 17(3), 255-273. doi: 10.1080/13548506.2011.608806

Prewitt, S. L., Hannon, J. C., \& Brusseau, T. A. (2013). Children and pedometers: A study in reactivity and knowledge. International Journal of Exercise Science, 6(3), $230-235$.

Pulgaron, E. R., Patino-Fernandez, A. M., Sanchez, J., Carrillo, A., \& Delamater, A. M. (2013). Hispanic children and the obesity epidemic: Exploring the role of abuelas. Fam Syst Health, 31(3), 274-279. doi: 10.1037/a0034208

Reilly, J. J. (2002). Assessment of childhood obesity: National reference data or international approach? Obes Res, 10(8), 838-840. doi: 10.1038/oby.2002.113 
Reilly, J. J. (2005). Descriptive epidemiology and health consequences of childhood obesity. Best Practice \& Research Clinical Endocrinology \& Metabolism, 19(3), $327-341$.

Reilly, J. J., Methven, E., McDowell, Z. C., Hacking, B., Alexander, D., Stewart, L., \& Kelnar, C. J. H. (2003). Health consequences of obesity. Archives of Disease in Childhood, 88(9), 748-752.

Rhodes, R. E., Macdonald, H. M., \& McKay, H. A. (2006). Predicting physical activity intention and behaviour among children in a longitudinal sample. Social Science \& Medicine, 62(12), 3146-3156. doi: S0277-9536(05)00658-1

Rice, M., \& Broome, M. E. (2004). Incentives for children in research. Journal of Nursing Scholarship, 36(2), 167-172. doi: 10.1111/j.1547-5069.2004.04030.x

Ridley, K., Ainsworth, B. E., \& Olds, T. S. (2008). Development of a compendium of energy expenditures for youth. International Journal of Behavioral Nutrition and Physical Activity, 5(45), 1-8. doi: 10.1186/1479-5868-5-45

Rimal, R. N. (2001). Longitudinal influences of knowledge and self-efficacy on exercise behavior: Tests of a mutual reinforcement model. Journal of Health Psychology, $6(1), 31-46$.

Roberts, V., Maddison, R., Magnusson, J., \& Prapavessis, H. (2010). Adolescent physical activity: Does implementation intention have a role? Journal of Physical Activity and Health, 7(4), 497-507.

Rodgers, C. C. (2007). Health promotion of the school-age child and family. Missouri, St. Louis: Westline Industrial Drive. 
Roemmich, J. N., Epstein, L. H., Raja, S., \& Yin, L. (2007). The neighborhood and home environments: Disparate relationships with physical activity and sedentary behaviors in youth. Ann Behav Med, 33(1), 29-38. doi:

$10.1207 /$ s15324796abm3301_4

Rose, E., Larkin, D., Hands, B., Howard, B., \& Parker, H. (2009). Evidence for the validity of the Children's Attraction to Physical Activity questionnaire (CAPA) with young children. Journal of Science and Medicine in Sport/Sports Medicine Australia, 12(5), 573-578. doi: 10.1016/j.jsams.2009.05.009

Rowe, D. A., Mahar, M. I., Raedeke, T. D., \& Lore, J. (2004). Measuring physical activity in children with pedometers: Reliability, reactivity, and replacement of missing data. Pediatric Exercise Science, 16(4), 343-354.

Rowe, D. A., Raedeke, T. D., Wiersma, L. D., \& Mahar, M. T. (2007). Investigating the youth physical activity promotion model: Internal structure and external validity evidence for a potential measurement model. Pediatric Exercise Science, 19(4), 420-435.

Ryan, R. M., \& Deci, E. L. (2000). Self-determination theory and the facilitation of intrinsic motivation, social development, and well-being. American Psychologist, $55(1), 68-78$.

Saha, A. K., Sarkar, N., \& Chatterjee, T. (2011). Health consequences of childhood obesity. Indian Journal of Pediatrics, 78(11), 1349-1355.

Sallis, J. F. (1991). Self-report measures of children's physical activity. Journal of School Health, 61(5), 215-219. 
Sallis, J. F. (2010). Effects of the built environment on physical activity level. In C.

Bouchard \& P. T. Katzmarzyk (Eds.), Physical Actiivty and Obesity. Champaign, IL: Thomson-Shore, Inc.

Sallis, J. F., Bauman, A., \& Pratt, M. (1998). Environmental and policy interventions to promote physical activity. American Journal of Preventive Medicine, 15, 379-397.

Sallis, J. F., Buono, M. J., Roby, J. J., Micale, F. G., \& Nelson, J. A. (1993). Seven-day recall and other physical activity self-reports in children and adolescents. Medicine and Science in Sports and Exercise, 25(1), 99-108.

Sallis, J. F., Cervero, R. B., Ascher, W., Henderson, K. A., Kraft, M. K., \& Kerr, J. (2006). An ecological approach to creating active living communities. Annual Review of Public Health, 27, 297-322.

Sallis, J. F., McKenzie, T. L., Kolody, B., Lewis, M., Marshall, S., \& Rosengard, P. (1999). Effects of health-related physical education on academic achievement: Project SPARK. Research Quarterly for Exercise and Sport, 70(2), 127-134.

Sallis, J. F., Prochaska, J. J., \& Taylor, W. C. (2000). A review of correlates of physical activity of children and adolescents. Medicine and Science in Sports and Exercise, $32(5), 963-975$.

Sallis, J. F., Prochaska, J. J., Taylor, W. C., Hill, J. O., \& Geraci, J. C. (1999). Correlates of physical activity in a national sample of girls and boys in grades 4 through 12 . Health Psychology, 18(4), 410-415.

Sallis, J. F., Strikmiller, P. K., Harsha, D. W., Feldman, H. A., Ehlinger, S., Stone, E. J., . . Woods, S. (1996). Validation of interviewer- and self-administered 
physical activity checklists for fifth grade students. Medicine and Science in Sports and Exercise, 28(7), 840-851.

Salmon, J., Brown, H., \& Hume, C. (2009). Effects of strategies to promote children's physical activity on potential mediators. Int J Obes (Lond), 33(Suppl 1), S66-S73. doi: 10.1038/ijo.2009.21

Saunders, R. P., Pate, R. R., Felton, G., Dowda, M., Weinrich, M. C., Ward, D. S., . . Baranowski, T. (1997). Development of questionnaires to measure psychosocial influences on children's physical activity. Preventive Medicine, 26(2), 241-247.

Stephanie von Hinke Kessler Scholder. (2008). Maternal employment and overweight children: Does timing matter? Health Economics, 17(8), 889-906. doi: 10.1002/Hec. 1357

Scommegna, P. (2012). More U.S. children raised by grandparents. Retrieved November 08, 2013, from http://www.prb.org/Publications/Articles/2012/US-childrengrandparents.aspx

Singh, G. K., Kogan, M. D., Van Dyck, P. C., \& Siahpush, M. (2008). Racial/ethnic, socioeconomic, and behavioral determinants of childhood and adolescent obesity in the United States: Analyzing independent and joint associations. Annals of Epidemiology, 18(9), 682-695.

Singh, G. K., Siahpush, M., \& Kogan, M. D. (2010). Rising social inequalities in US childhood obesity, 2003-2007. Ann Epidemiol, 20(1), 40-52. doi: 10.1016/j.annepidem.2009.09.008

Sirard, J. R., \& Pate, R. R. (2001). Physical activity assessment in children and adolescents. Sports Medicine, 31(6), 439-454. 
Sleddens, E. F. C., Kremers, S. P. J., Hughes, S. O., Cross, M. B., Thijs, C., De Vries, N. K., \& O'Connor, T. M. (2012). Physical activity parenting: A systematic review of questionnaires and their associations with child activity levels. Obesity Reviews, 13(11), 1015-1033.

Slyper, A. H. (2004). The pediatric obesity epidemic: Causes and controversies. Journal of Clinical Endocrinology \& Metabolism, 89(6), 2540-2547. doi:

10.1210/jc.2003-031449

Smith, M. Z., \& Burton, L. J. (2005). Parental influences on the physical activity behavior of children of various ethnic backgrounds. Research Quarterly for Exercise and Sport, 76(1), A50-A51.

Smith, N. J., \& Lounsbery, M. (2009). Promoting physical education: The link to academic achievement. Journal of Physical Education, Recreation \& Dance, 80(1), 39-43.

Soto, C., \& White, J. H. (2010). School health initiatives and childhood obesity: BMI screening and reporting. Policy Polit Nurs Pract, 11(2), 108-114. doi:

$10.1177 / 1527154410374218$

SPARK. (2012). Physical education. Retrieved November 13, 2012, from http://www.sparkpe.org/physical-education/

Spence, J. C., Blanchard, C. M., Clark, M., Plotnikoff, R. C., Storey, K. E., \& McCargar, L. (2010). The role of self-efficacy in explaining gender differences in physical activity among adolescents: A multilevel analysis. Journal of Physical Activity and Health, 7(2), 176-183. 
Standage, M., Duda, J. L., \& Ntoumanis, N. (2003). A model of contextual motivation in physical education: Using constructs from self-determination and achievement goal theories to predict physical activity intentions. Journal of Educational Psychology, 95(1), 97-110. doi: 10.1037/0022-0663.95.1.97

Standage, M., Duda, J. L., \& Ntoumanis, N. (2005). A test of self-determination theory in school physical education. British Journal of Educational Psychology, 75, 411433. doi: $10.1348 / 000709904 \times 22359$

Steinbeck, K. S. (2001). The importance of physical activity in the prevention of overweight and obesity in childhood: A review and an opinion. Obesity Reviews, 2(2), 117-130.

Story, M. T., Neumark-Stzainer, D. R., Sherwood, N. E., Holt, K., Sofka, D., Trowbridge, F. L., \& Barlow, S. E. (2002). Management of child and adolescent obesity: Attitudes, barriers, skills, and training needs among health care professionals. Pediatrics, 110(1 Pt 2), 210-214.

Strauss, R. S. (2000). Childhood obesity and self-esteem. Pediatrics, 105(1), e15.

Strauss, R. S., Loree, R., Burak, G., Rozdilsky, D., \& Colin, M. (2000). Social and cognitive determinants of physical activity in children and young adolescents. Obesity Research, 8, S18.

Strong, W. B., Malina, R. M., Blimkie, C. J. R., Daniels, S. R., Dishman, R. K., Gutin, B., ... Trudeau, F. (2005). Evidence based physical activity for school-age youth. Journal of Pediatrics, 146(6), 732-737.

Suhr, D. (2012). Step your way through path analysis. Retrieved February 11, 2013, from 
http://www.wuss.org/proceedings08/08WUSS\%20Proceedings/papers/pos/pos04. pdf

Tabachnick, B. G., \& Fidell, L. S. (2007). Using multivariate statistics. Boston: Pearson Education. Inc.

The Annie E. Casey Foundation. (2013). Public school students receiving free or reduced price lunches (percent) - 2011. Retrieved April 03, 2013, from http://datacenter.kidscount.org/data/bystate/Rankings.aspx?state=IN\&ind=5187

The National Conference of State Legislatures. (2010). Childhood obesity: 2010 update of legislative policy options. Retrieved October 30, 2013, from http://www.ncsl.org/research/health/childhood-obesity-2010.aspx

The Office of Fair Housing and Equal Opportunity. (2012). Demographics summary. Retrieved February 6, 2013, from http://www.clrsearch.com

The White House: Office of the First Lady. (2010). First Lady Michelle Obama launches Let's Move: America's move to raise a healthier generation of kids. Retrieved December 19, 2012, from http://www.whitehouse.gov/the-press-office/first-ladymichelle-obama-launches-lets-move-americas-move-raise-a-healthier-genera The White House: Office of the Press Secretary. (2010). President Obama signs Healthy, Hunger-Free Kids Act of 2010 into law. Retrieved July 15, 2012, from http://www.whitehouse.gov/the-press-office/2010/12/13/president-obama-signshealthy-hunger-free-kids-act-2010-law

Thompson, D., Baranowski, T., Cullen, K., Watson, K., Liu, Y., Canada, A., . . Zakeri, I. (2008). Food, fun, and fitness internet program for girls: Pilot evaluation of an e- 
health youth obesity prevention program examining predictors of obesity. Preventive Medicine, 47(5), 494-497. doi: 10.1016/j.ypmed.2008.07.014

Thompson, J. W., \& Card-Higginson, P. (2009). Arkansas' experience: Statewide surveillance and parental information on the child obesity epidemic. Pediatrics, 124(Suppl 1), S73-S82. doi: 10.1542/peds.2008-3586J

Timperio, A., Salmon, J., \& Ball, K. (2004). Evidence-based strategies to promote physical activity among children, adolescents and young adults: Review and update. Journal of Science and Medicine in Sport, 7(1 Suppl), 20-29.

Trapp, G., Giles-Corti, B., Christian, H., Timperio, A. F., McCormack, G. R., Bulsara, M., \& Villanueva, K. (2013). Driving down daily step counts: The impact of being driven to school on physical activity and sedentary behavior. Pediatric Exercise Science, 25(3), 337-346.

Treuth, M. S., Hou, N., Young, D. R., \& Maynard, L. M. (2005). Validity and reliability of the Fels physical activity questionnaire for children. Medicine and Science in Sports and Exercise, 37(3), 488-495.

Trinh, A., Campbell, M., Ukoumunne, O. C., Gerner, B., \& Wake, M. (2013). Physical activity and 3-year BMI change in overweight and obese children. Pediatrics, 131(2), e470-e477. doi: 10.1542/peds.2012-1092

Troiano, R. P., Berrigan, D., Dodd, K. W., Masse, L. C., Tilert, T., \& McDowell, M. (2008). Physical activity in the United States measured by accelerometer. Medicine and Science in Sports and Exercise, 40(1), 181-188. doi: 10.1249/mss.0b013e31815a51b3 
Trost, S. G., Kerr, L. M., Ward, D. S., \& Pate, R. R. (2001). Physical activity and determinants of physical activity in obese and non-obese children. International Journal of Obesity and Related Metabolic Disorders, 25(6), 822-829. doi:

10.1038/sj.ijo.0801621

Trost, S. G., Sallis, J. F., Pate, R. R., Freedson, P. S., Taylor, W. C., \& Dowda, M. (2003). Evaluating a model of parental influence on youth physical activity. Am J Prev Med, 25(4), 277-282.

Tudor-Locke, C., Craig, C. L., Beets, M. W., Belton, S., Cardon, G. M., Duncan, S., . . . Blair, S. N. (2011). How many steps/day are enough? For children and adolescents. International Journal of Behavioral Nutrition and Physical Activity, 8(78), 1-14. doi: 10.1186/1479-5868-8-78

Tudor-Locke, C., Johnson, W. D., \& Katzmarzyk, P. T. (2010). Accelerometerdetermined steps per day in US children and youth. Med Sci Sports Exerc, 42(12), 2244-2250. doi: 10.1249/MSS.0b013e3181e32d7f

U.S. Preventive Services Task Force. (2010). Screening for obesity in children and adolescents: US Preventive Services Task Force recommendation statement. Pediatrics, 125(2), 361-367. doi: 10.1542/peds.2009-2037

U.S. Department of Health \& Human Services. (2008). Physical activity guidelines for americans. Retrieved April 03, 2013, from http://www.health.gov/paguidelines/guidelines/chapter3.aspx

U.S. Department of Health \& Human Services, National Institute of Health, \& National Heart, Lung, and Blood Institute. (2011). Expert panel on integrated guidelines 
for cardiovascular health and risk reduction in children and adolescents: Summary report. Pediatrics, 12(Suppl 5), S213-S256.

U.S. Department of Health and Human Services. (2009). 2008 Physical Activity Guidelines for Americans. Washington: HHS.

U.S. Department of Health and Human Services. (2010). Healthy People 2020. Retrieved January 29, 2013, from http://healthypeople.gov/2020/

U.S. Department of Health and Human Services, \& Centers for Disease Control and Prevention. (2012). Youth risk behavior surveillance - United States, 2011. Retrieved November 10, 2012, from http://www.cdc.gov/mmwr/pdf/ss/ss6104.pdf U.S. Census Bureau. (2013). State \& County QuickFacts: Clark County, Indiana. Retrieved January 24, 2013, from http://quickfacts.census.gov/qfd/states/18/18019.html

Van der Horst, K., Paw, M. J, Twisk, J. W, \& Van Mechelen, W. (2007). A brief review on correlates of physical activity and sedentariness in youth. Medicine and Science in Sports and Exercise, 39(8), 1241-1250. doi:

10.1249/mss.0b013e318059bf35

Van Domelen, D. R., Koster, A., Caserotti, P., Brychta, R. J., Chen, K. Y., McClain, J. J., ... Harris, T. B. (2011). Employment and physical activity in the U.S. Am J Prev Med, 41(2), 136-145. doi: 10.1016/j.amepre.2011.03.019

Vandivere, S., Tout, K., Zaslow, M., Calkins, J., \& Capizzano, J. (2003). Unsupervised time: Family and child factors associated with self-care. Retrieved October 30, 2013, from http://www.urban.org/UploadedPDF/310894_OP71.pdf 
Vanhees, L., Lefevre, J., Philippaerts, R., Martens, M., Huygens, W., Troosters, T., \& Beunen, G. (2005). How to assess physical activity? How to assess physical fitness? European Journal of Cardiovascular Prevention and Rehabilitation, 12(2), 102-114.

Vierling, K. K., Standage, M., \& Treasure, D. C. (2007). Predicting attitudes and physical activity in an "at-risk" minority youth sample: A test of self-determination theory. Psychology of Sport and Exercise, 8(5), 795-817. doi:

10.1016/j.psychsport.2006.12.006

Wang, F., \& Veugelers, P. J. (2008). Self-esteem and cognitive development in the era of the childhood obesity epidemic. Obesity Reviews, 9(6), 615-623.

Wang, G., \& Dietz, W. H. (2002). Economic burden of obesity in youths aged 6 to 17 years: 1979-1999. Pediatrics, 109(5), E81-E81.

Wang, Y., Beydoun, M. A., Liang, L., Caballero, B., \& Kumanyika, S. K. (2008). Will all Americans become overweight or obese? Estimating the progression and cost of the US obesity epidemic. Obesity, 16(10), 2323-2330.

Wang, Y., \& Lobstein, T. (2006). Worldwide trends in childhood overweight and obesity. Int J Pediatr Obes, 1(1), 11-25.

Wareham, N. J., van Sluijs, E. M. F., \& Ekelund, U. (2005). Physical activity and obesity prevention: A review of the current evidence. Proceedings of the Nutrition Society, 64(2), 229-247.

Watkinson, C., van Sluijs, E. M. F., Sutton, S., Hardeman, W., Corder, K., \& Griffin, S. J. (2010). Overestimation of physical activity level is associated with lower BMI: A 
cross-sectional analysis. International Journal of Behavioral Nutrition and Physical Activity, 7(68), 1-9. doi: 10.1186/1479-5868-7-68

Wattanasit, P. (2009). Determinants of physical activity in Thai adolescents: Testing the Youth Physical Activity Promotion Model. (Doctor Dissertation, Prince of Songkla University). Retrieved November 23, 2013 from kb.psu.ac.th/psukb/bitstream/2010/5877/1/313873.pdf

Weijters, B., Baumgartner, H., \& Schillewaert, N. (2013). Reversed item bias: An integrative model. Psychological Methods, 18(3), 320-334. doi: $10.1037 / \mathrm{A} 0032121$

Weir, J. P. (2005). Quantifying test-retest reliability using the intraclass correlation coefficient and the SEM. Journal of Strength and Conditioning Research/National Strength \& Conditioning Association, 19(1), 231-240.

Weir, L. A., Etelson, D., \& Brand, D. A. (2006). Parents' perceptions of neighborhood safety and children's physical activity. Preventive Medicine, 43(3), 212-217. doi: 10.1016/j.ypmed.2006.03.024

Welk, G. J. (1999). The Youth Physical Activity Promotion Model: A conceptual bridge between theory and practice. Quest, 51(1), 5-23.

Welk, G. J. (2002). Physical activity assessments for health-related research. New Zealand: Human Kinetics Publishers, Inc. .

Welk, G. J., Corbin, C. B., \& Dale, D. (2000). Measurement issues in the assessment of physical activity in children. Research Quarterly for Exercise and Sport, 71(2 Suppl), S59-S73. 
Welk, G. J., Wood, K., \& Morss, G. (2003). Parental influences on physical activity in children: An exploration of potential mechanisms. Pediatric Exercise Science, 15(1), 19-33.

West, S. G., Finch, J. F., \& Curran, P. J. (1995). Structural equation models with nonnormal variables: Problems and remedies. In D. R. Heise (Ed.), Structural equation modeling: Concepts, issues, and applications (pp. 56-75). Thousand Oaks, CA: Sage.

White, C. (2013). Greater Clark to lease 8,700 Chromebooks for students, staff. Retrieved November 02, 2013, from http://www.courierjournal.com/article/20130417/NEWS02/304170061/

Wiecha, J. L., Hall, G., Gannett, E., \& Roth, B. (2011). National afterschool association standards for healthy eating and physical activity. Retrieved October 30, 2013, from http://www.niost.org/Standards-and-Guidelines/national-afterschoolassociation-standards-for-healthy-eating-and-physical-activity-in-out-of-schooltime-programs

Williams, C. L., Hayman, L. L., Daniels, S. R., Robinson, T. N., Steinberger, J., Paridon, S. M., \& Bazzarre, T. (2002). Cardiovascular health in childhood: A statement for health professionals from the Committee on Atherosclerosis, Hypertension, and Obesity in the Young (AHOY) of the Council on cardiovascular disease in the Young, American Heart Association. Circulation, 106(1), 143-160.

Williams, S. L., \& French, D. P. (2011). What are the most effective intervention techniques for changing physical activity self-efficacy and physical activity 
behaviour--and are they the same? Health Education Research, 26(2), 308-322. doi: 10.1093/her/cyr005

Zaki, R., Bulgiba, A., Nordin, N., \& Azina-Ismail, N. (2013). A systematic review of statistical methods used to test for reliability of medical instruments measuring continuous variables. Iranian Journal of Basic Medical Sciences, 16(6), 803-807. 


\section{APPENDICES}

\section{Appendix A}

Reliability and Validity of Self-report Instruments for Assessing Physical Activity in Children

\begin{tabular}{|c|c|c|c|c|}
\hline Instrument & Characteristics & Reliability & Validity & Reference \\
\hline $\begin{array}{l}\text { Self-Report of } \\
\text { Aerobic Activity }\end{array}$ & $\begin{array}{l}\text { - } \begin{array}{l}\text { Self-administered, daily } \\
\text { recall. }\end{array} \\
\text { - Assess duration of } \\
\text { participation in different } \\
\text { types of activities. } \\
\text { - Energy expenditure can } \\
\text { be calculated from data } \\
\text { on duration and intensity } \\
\text { of activity. } \\
\text { - Does not explore time } \\
\text { spent engaged in } \\
\text { sedentary behaviors. }\end{array}$ & $\begin{array}{l}\text { Overall: Unknown } \\
\text { Sample: Children, Grade } 3 \\
\text { to } 6(N=78) \\
\text { - Compared two formats } \\
\text { of scale - daily self- } \\
\text { report and weekly } \\
\text { retrospective report } \\
\text { - No meaningful results } \\
\text { were obtained among } \\
\text { children. }\end{array}$ & $\begin{array}{l}\text { Overall: Acceptable } \\
\text { Sample: Children, Grade } 3 \text { to } 6 \\
(N=24) \\
\text { - The daily self-report form was } \\
\text { compared with continuous } \\
\text { observation of behaviors by } \\
\text { investigators. } \\
\text { - The activity level recorded by } \\
\text { the daily self-report form was } \\
\text { consistent with the activity } \\
\text { level observed by investigator. }\end{array}$ & $\begin{array}{l}\text { (Baranowski et } \\
\text { al., 1984) }\end{array}$ \\
\hline
\end{tabular}




\begin{tabular}{|c|c|c|c|c|}
\hline Instrument & Characteristics & Reliability & Validity & Reference \\
\hline $\begin{array}{l}\text { Interviewer- } \\
\text { administered } \\
\text { Physical Activity } \\
\text { Checklists (PACI) }\end{array}$ & $\begin{array}{l}\text { - Interviewer- } \\
\text { administered, previous } \\
\text { day recall of physical } \\
\text { activity before, during } \\
\text { and after school. } \\
\text { - Provides a total estimate } \\
\text { of the number of } \\
\text { minutes spent engaged } \\
\text { in activities. } \\
\text { - Measures time spent } \\
\text { engaged in sedentary } \\
\text { behaviors. } \\
\text { - Measures time spent in } \\
\text { moderate to vigorous } \\
\text { activity. } \\
\text { - MET score is calculated } \\
\text { from data on time and } \\
\text { intensity of activities. }\end{array}$ & $\begin{array}{l}\text { Overall: Moderate } \\
\text { Sample: Children, Grade } 5 \\
(N=97) \\
\text { - Intraclass correlations } \\
\text { based on comparisons of } \\
\text { the results from the self- } \\
\text { administered and } \\
\text { interviewer- } \\
\text { administered recalls } \\
\text { where the only } \\
\text { difference between the } \\
\text { two was the method of } \\
\text { administration: } \\
\text { ICC (range): } 0.64-0.79 .\end{array}$ & $\begin{array}{l}\text { Overall: Low to Moderate } \\
\text { Sample: Children, Grade } 5 \\
(N=97) \\
\text { Validated against: } \\
\text { - Heart rate monitoring and } \\
\text { number of activities ( } r=0.22) \text {. } \\
\text { - Heart rate monitoring and } \\
\text { minutes of moderate to } \\
\text { vigorous activity (MVPA) } \\
(r=0.50) \text {. } \\
\text { - Heart rate monitoring and } \\
\text { MVPA METs }(r=0.52) \text {. } \\
\text { - Accelerometer and number of } \\
\text { Activities }(r=0.10) \text {. } \\
\text { - Accelerometer and minutes of } \\
\text { MVPA ( } r=0.32) \text {. } \\
\text { - Accelerometer and MVPA } \\
\text { METs }(r=0.36) \text {. }\end{array}$ & $\begin{array}{l}\text { (Sallis et al., } \\
\text { 1996) }\end{array}$ \\
\hline
\end{tabular}




\begin{tabular}{|c|c|c|c|c|}
\hline Instrument & Characteristics & Reliability & Validity & Reference \\
\hline $\begin{array}{l}\text { Self-administered } \\
\text { Physical Activity } \\
\text { Checklists } \\
\text { (SAPAC) }\end{array}$ & $\begin{array}{l}\text { - Self-administered, } \\
\text { previous day recall of } \\
\text { physical activity before, } \\
\text { during and after school. } \\
\text { - Provides a total estimate } \\
\text { of the number of } \\
\text { minutes spent engaged } \\
\text { in activities. } \\
\text { - Measures time spent } \\
\text { engaged in sedentary } \\
\text { behaviors. } \\
\text { - Measures time spent in } \\
\text { moderate to vigorous } \\
\text { activity. } \\
\text { - MET score is calculated } \\
\text { from data on time and } \\
\text { intensity of activities. }\end{array}$ & $\begin{array}{l}\text { Overall: Moderate } \\
\text { Sample: Children, Grade } 5 \\
\text { ( } N=97) \\
\text { - Intraclass correlations } \\
\text { based on comparisons of } \\
\text { the results from the self- } \\
\text { administered and } \\
\text { interviewer- } \\
\text { administered recalls } \\
\text { where the only } \\
\text { difference between the } \\
\text { two was the method of } \\
\text { administration: } \\
\text { ICC (range): } 0.64-0.79 \text {. }\end{array}$ & $\begin{array}{l}\text { Overall: Low to Moderate } \\
\text { Sample: Children, Grade } 5 \\
(N=97) \\
\text { Validated against: } \\
\text { - Heart rate monitoring and } \\
\text { number of activities }(r=0.28) \text {. } \\
\text { - Heart rate monitoring and } \\
\text { minutes of moderate to } \\
\text { vigorous activity (MVPA) } \\
(r=0.58) \text {. } \\
\text { - Heart rate monitoring and } \\
\text { MVPA METs }(r=0.60) \text {. } \\
\text { - Accelerometer and number of } \\
\text { Activities }(r=0.02) \text {. } \\
\text { - Accelerometer and minutes of } \\
\text { MVPA }(r=0.30) \text {. } \\
\text { - Accelerometer and MVPA } \\
\text { METs }(r=0.32) \text {. }\end{array}$ & $\begin{array}{l}\text { (Sallis et al., } \\
1996)\end{array}$ \\
\hline
\end{tabular}




\begin{tabular}{|c|c|c|c|c|}
\hline Instrument & Characteristics & Reliability & Validity & References \\
\hline $\begin{array}{l}\text { Seven-day } \\
\text { Physical Activity } \\
\text { Recall (PAR) }\end{array}$ & $\begin{array}{l}\text { - Interview-administered, } \\
\text { 7-day recall of physical } \\
\text { activity at various } \\
\text { intensity levels-sleep, } \\
\text { moderate, hard and very } \\
\text { hard. } \\
\text { - Assess time spent } \\
\text { engaged in sedentary, } \\
\text { moderate, hard and very } \\
\text { hard activity on } \\
\text { weekday and weekend. } \\
\text { - MET score is calculated } \\
\text { from data on time and } \\
\text { intensity of activities. }\end{array}$ & $\begin{array}{l}\text { Overall: Low to Moderate } \\
\text { Sample: Children, Grade } 5 \\
(N=36) \\
\text { Test-retest ( } 2 \text { week): } \\
\text { - } r=0.47 \text { (Total) } \\
\text { - } r=0.51 \text { (Sleep) } \\
\text { - } r=0.13 \text { (Moderate) } \\
\text { - } r=0.11 \text { (Hard) } \\
\text { - } r=0.36 \text { (Very Hard) }\end{array}$ & $\begin{array}{l}\text { Overall: Moderate } \\
\text { Sample: Children, Grade } 5 \\
(N=36) \\
\text { Hours of recalled very hard } \\
\text { activity were significantly } \\
\text { correlated with: } \\
\text { - Minutes in intervals with } \\
\text { HR } \geq 140(r=0.33) \text {. } \\
\text { - Minutes in intervals with } \\
\text { HR } \geq 160(r=0.29) \text {. }\end{array}$ & $\begin{array}{l}\text { (Sallis, Buono, } \\
\text { Roby, Micale, \& } \\
\text { Nelson, 1993) }\end{array}$ \\
\hline
\end{tabular}




\begin{tabular}{|c|c|c|c|c|}
\hline Instrument & Characteristics & Reliability & Validity & References \\
\hline $\begin{array}{l}\text { Physical Activity } \\
\text { Questionnaire for } \\
\text { Older Children } \\
\text { (PAQ-C) }\end{array}$ & $\begin{array}{l}\text { - Self-administered, 7- } \\
\text { day recall. } \\
\text { - Activities are presented } \\
\text { on a 5-point Likert } \\
\text { scale. } \\
\text { - Assesses frequency of } \\
\text { participation in } \\
\text { different types of } \\
\text { activities rather than } \\
\text { duration. } \\
\text { - Includes activities } \\
\text { performed both in and } \\
\text { out of school. } \\
\text { - Segregates weekday } \\
\text { participation from } \\
\text { weekend participation. } \\
\text { - Does not explore time } \\
\text { spent engaged in } \\
\text { sedentary behaviors. }\end{array}$ & $\begin{array}{l}\text { Overall: High } \\
\text { Sample: Children, } \\
\text { aged } 9 \text { to } 15 \text { Years } \\
(N=215) \\
\text { Cronbach's alpha: } \\
-\quad 0.83 \text { (all) } \\
-\quad 0.83 \text { (females) } \\
-\quad 0.80 \text { (males) } \\
\text { Sample: Children, } \\
\text { aged } 9 \text { to } 14 \text { Years ( } N \\
=84 \text { ) } \\
\text { Cronbach's alpha: } \\
-\quad 0.79 \text { (Time } 1) \\
-\quad 0.89 \text { (Time 2) } \\
\text { Test-retest (1-week): } \\
-\quad r=0.75 \text { (males) } \\
-\quad r=0.82 \text { (females) }\end{array}$ & $\begin{array}{l}\text { Overall: Moderate } \\
\text { Sample: Children, aged } 8 \text { to } 13 \text { Years } \\
(N=83 / 97) \\
\text { Validated against: } \\
\text { - Activity rating ( } r=0.57 / 0.63) \text {. } \\
\text { - Week summation of Moderate to } \\
\text { Vigorous Physical Activity ( } r=0.53) \text {. } \\
\text { - Athletic competence ( } r=0.48) \text {. } \\
\text { - Teacher's rating of physical activity } \\
\text { ( } r=0.45) \text {. } \\
\text { - Leisure Time Exercise Questionnaire } \\
(r=0.41) \text {. } \\
\text { - Canadian home fitness test }(r=0.28) \text {. } \\
\text { - Seven-day Recall Interview }(r=0.46) \text {. } \\
\text { - Caltrac motion sensor }(r=0.39) .\end{array}$ & $\begin{array}{l}\text { (Crocker et al., } \\
1997 ; \\
\text { Kowalski et al., } \\
1997 \text { ) }\end{array}$ \\
\hline
\end{tabular}




\begin{tabular}{|c|c|c|c|c|}
\hline Instrument & Characteristics & Reliability & Validity & Reference \\
\hline $\begin{array}{l}\text { Fels Physical } \\
\text { Activity } \\
\text { Questionnaire } \\
\text { (PAQ) }\end{array}$ & $\begin{array}{l}\text { - Self-administered scale } \\
\text { to assess habitual } \\
\text { physical activity. } \\
\text { - Three "open" questions } \\
\text { assessing the types and } \\
\text { frequency of activities in } \\
\text { the last year. } \\
\text { - Five Likert-scale } \\
\text { questions evaluating } \\
\text { physical activity. } \\
\text { - Provide a total physical } \\
\text { activity score along with } \\
\text { scores for catalogs of } \\
\text { sport, leisure and work } \\
\text { activity. }\end{array}$ & 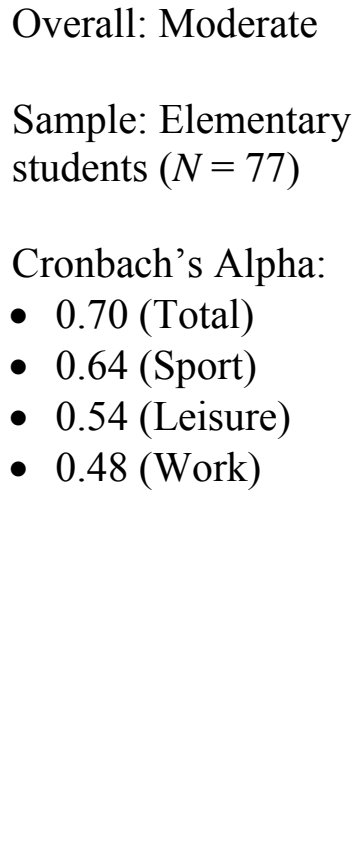 & $\begin{array}{l}\text { Overall: Low to Moderate } \\
\text { Sample: Elementary students } \\
(N=77) \\
\text { Accelerometer were significantly } \\
\text { correlated with: } \\
\text { - Total score }(r=0.34) \\
\text { - Sport score }(r=0.32) \\
\text { - Leisure score }(r=0.28)\end{array}$ & $\begin{array}{l}\text { (Treuth, Hou, } \\
\text { Young, \& } \\
\text { Maynard, 2005) }\end{array}$ \\
\hline
\end{tabular}




\section{Appendix B}

Demographic Information Questionnaire

1. Your child's age: Years old

2. Your child's sex:

Male

Female

3. Your child's ethnicity: $\square$ Hispanic $\square$ Non-Hispanic

4. Your child's race:

$\square$ White or Caucasian $\square$ Black or African American $\square$ Asian/Pacific Island

$\square$ American Indian and Alaska Native $\quad \square$ Other

5. How many children do you have?

6. What is your marital status?

$\square$ Married/partnered $\quad \square$ Separated, divorced or widowed $\quad \square$ Single

7. How much is the annual family income last year?

$\square$ Under \$19,999 $\quad \square \$ 20,000-\$ 29,999$

$\square 30,000-\$ 49,999 \quad \square \$ 50,000$ or above

8. Is father employed?
$\square$ Full Time
$\square$ Part Time
$\square$ No

9. Is mother employed?
$\square$ Full Time
$\square$ Part Time
$\square$ No

10. What is the father's education level?

Less than high school graduate

$\square$ High school graduate 
$\square$ Some college (at least one year or specialized training)

$\square$ Technical school or community college degree

$\square$ Bachelor's Degree

$\square$ Graduate or professional degree

11. What is the mother's education level?

$\square$ Less than high school graduate

$\square$ High school graduate

$\square$ Some college (at least one year or specialized training)

$\square$ Technical school or community college degree

$\square$ Bachelor's Degree

$\square$ Graduate or professional degree

12. During the school year, how does your child go to school for most of the days?

$\square$ Walk $\quad \square$ Bicycle $\quad \square$ School Bus $\quad \square$ Car

13. During the school year, what following activities does your child do?

$\square$ Watching TV/DVDs $\quad \square$ Playing video games $\quad \square$ Using computer

14. During the school year, how many minutes per week does your child spend on the above activities? Minutes 


\section{Appendix C}

2 to 20 years: Boys Body mass index-for-age percentiles
NAME RECORD \#

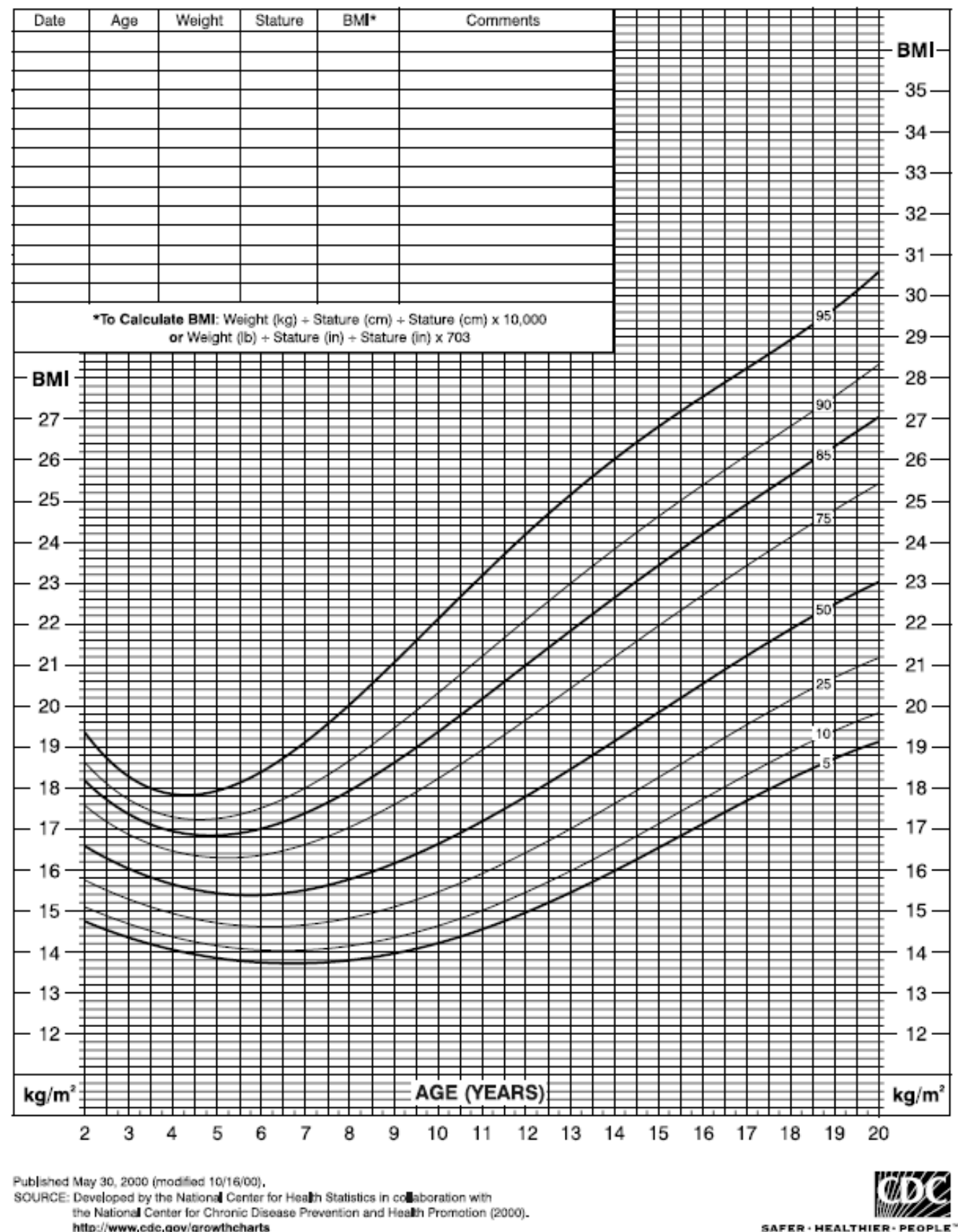




\section{Appendix D}

2 to 20 years: Girls Body mass index-for-age percentiles

NAME

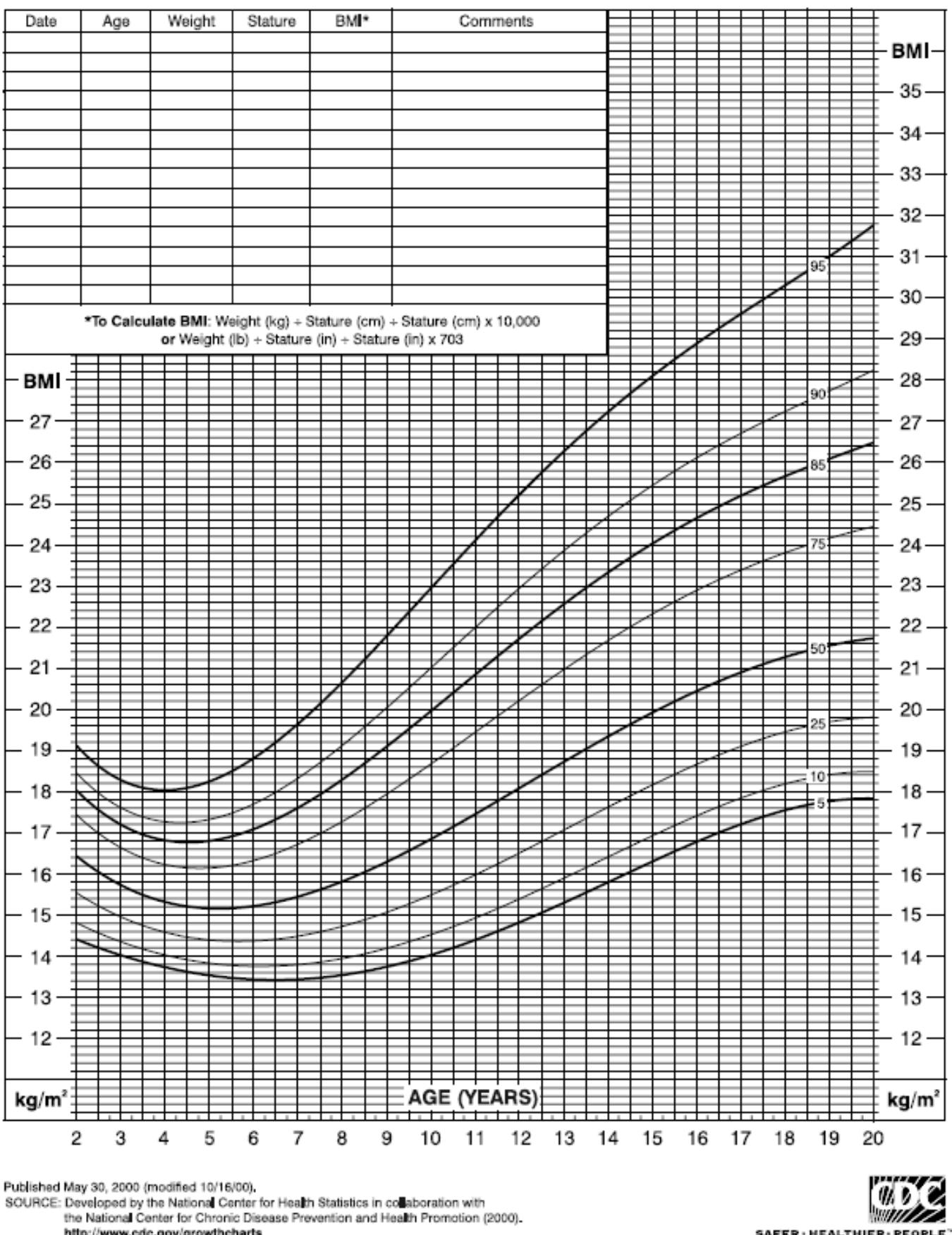

the Nationa Center for Chronic Disease Prevention and Healit Promotion (2000).

SAFER - HEALTHIER - PEOPLE 


\section{Appendix E}

\section{Pedometer Log}

Child's height:

Centimeters

Child's weight: Kilograms

Time and Date the pedometer was first worn by child

\section{Instructions:}

(1) Fill out the box below each night before you go to bed.

(2) Do not wear the pedometer in the shower, bath, or while swimming.

\begin{tabular}{|l|l|l|l|}
\hline Day & Date & Time Recorded & Number of Steps \\
\hline Day 1 & & & \\
\hline Day 2 & & & \\
\hline Day 3 & & & \\
\hline Day 4 & & & \\
\hline Day 5 & & & \\
\hline Day 6 & & & \\
\hline Day 7 & & & \\
\hline
\end{tabular}




\section{Parents and Guardians:}

\section{In the morning of every day on weekend:}

(1) Attach/Clip the pedometer to the belt or waistband of child's clothing as he or she gets dressed. This will ensure that the pedometer captures steps for as close to a full day as possible.

(2) Make sure the pedometer has been reset to zero steps.

\section{At night (before bedtime) of every day on weekend:}

(1) The pedometer should be removed before bedtime.

(2) Record the date, time, and number of steps the child took that day.

(3) Once that day's steps have been written down on this sheet, the pedometer should be reset to zero.

(4) Place the pedometer in a safe place where the child will remember to put it on the next day morning.

After Day 7, child should return the pedometer to the teacher/researcher.

If the child is sick during one or more days that he or she is supposed to be wearing the pedometer:

(1) Mark it in the third column (number of steps) of the above table.

(2) Steps do not need to be recorded if the child is sick. 


\section{Appendix F}

\section{Physical Activity Questionnaire for Older Children}

We are trying to find out about your level of physical activity from the last 7 days (in the last week). This includes sports or dances that make you sweat or make your legs feel tired, or games that make you breathe hard, like tag, skipping, running, climbing, and others.

\section{Remember:}

1. There are no right and wrong answers - this is not a test.

2. Please answer all the questions as honestly and accurately as you can - this is very important.

1. Physical activity in your spare time: Have you done any of the following activities in the past 7 days (last week)? If yes, how many times? (Mark only one circle per row.)

7 times

No $\quad 1-2 \quad 3-4 \quad 5-6 \quad$ or more

Skipping

Rowing/canoeing

In-line skating

Tag

Walking for exercise

Bicycling 
Jogging or running .

Aerobics

Swimming

Baseball, softball

Dance

Football

Badminton

Skateboarding

Soccer

Street hockey

Volleyball

Floor hockey

Basketball

Ice skating

Cross-country skiing

Ice hockey/ringette

Other: 
2. In the last 7 days, during your physical education (PE) classes, how often were you very active (playing hard, running, jumping, throwing)? (Check one only.)

I don't do PE

Hardly ever

Sometimes

Quite often

\section{Always}

3. In the last 7 days, what did you do most of the time at recess? (Check one only.)

Sat down (talking, reading, doing schoolwork).....

Stood around or walked around

Ran or played a little bit

Ran around and played quite a bit

Ran and played hard most of the time

4. In the last 7 days, what did you normally do at lunch (besides eating lunch)? (Check one only.)

Sat down (talking, reading, doing schoolwork)....

Stood around or walked around

Ran or played a little bit

Ran around and played quite a bit

Ran and played hard most of the time

5. In the last 7 days, on how many days right after school, did you do sports, dance, or play games in which you were very active? (Check one only.)

None 
1 time last week

2 or 3 times last week

4 times last week

5 times last week

6. In the last 7 days, on how many evenings did you do sports, dance, or play games in which you were very active? (Check one only.)

None

1 time last week

2 or 3 times last week

4 or 5 last week

6 or 7 times last week

7. On the last weekend, how many times did you do sports, dance, or play games in which you were very active? (Check one only.)

1 time

8. Which one of the following describes you best for the last 7 days? Read all five statements before deciding on the one answer that describes you.

A. All or most of my free time was spent doing things that involve little physical effort

B. I sometimes $(1-2$ times last week) did physical things in my free time 
(e.g. played sports, went running, swimming, bike riding, did aerobics) ..... $\square$

C. I often ( $3-4$ times last week) did physical things in my free time

D. I quite often (5 - 6 times last week) did physical things in my free time...

E. I very often (7 or more times last week) did physical things in my free tim

9. Mark how often you did physical activity (like playing sports, games, doing dance, or any other physical activity) for each day last week.

Little Very

None bit Medium Often often

Monday

Tuesday

Wednesday

Thursday

Friday

Saturday

Sunday

10. Were you sick last week, or did anything prevent you from doing your normal physical activities? (Check one.)

Yes

No

If Yes, what prevented you? 


\section{Appendix G}

\section{Physical Activity Self-Efficacy Scale}

\section{Instructions:}

1. Please put a check by "Yes" or "No" for each of the following sentences. "Yes" means that you agree with the sentence. "No" means that you do not agree with the sentence.

2. Remember that physical activity can be any play, game, sport, or exercise that gets you moving and breathing harder.

\section{There are no wrong answers.}

1. I think I can be physically active most days after school. Yes No

2. I think I can ask my parent or other adult to do physically active things with me. Yes No

3. I think I can be physically active after school even if I could watch TV or play video games instead. Yes No

4. I think I can be physically active after school even if my friends want me to do something else. Yes No

5. I think I can ask my parent or other adult to sign me up for a sport, dance, or other physical activity program. Yes No

6. I think I can be physically active even if it is very hot or cold outside. Yes No 
7. I think I can ask my best friend to be physically active with me.

Yes No

8. I think I can ask my parent or other adult to get me the equipment I need to be physically active. Yes No

9. I think I can ask my parent or other adult to take me to a physical activity or sport practice. Yes No

10. I think I can be physically active, even if I have a lot of homework. Yes No

11. I think I can be physically active even if I have to stay at home. Yes No

12. I think I have the skills I need to be physically active.

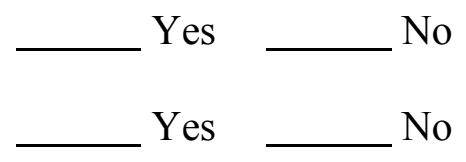

13. I think I can be physically active no matter how busy my day is. Yes No

14. I think I can be physically active no matter how tired I may feel. Yes No

15. I think I can be physically active even when I'd rather be doing something else. Yes

16. I think I can be physically active even if my friends don't want me to. Yes No

17. I think I can be physically active at least three times a week for the next 2 weeks. Yes No 


\section{Appendix H}

Physical Activity Enjoyment Scale

\begin{tabular}{|c|c|c|c|c|c|}
\hline \multicolumn{6}{|l|}{ When I am physically active: } \\
\hline \multirow{5}{*}{$\begin{array}{l}\text { 1. I enjoy it } \\
\text { 2. } 1 \text { feel bored } \\
\text { 3. I dislike it } \\
\text { 4. I find it pleasurable }\end{array}$} & $\begin{array}{c}\text { Disagree } \\
\text { a lot }\end{array}$ & Disagree & $\begin{array}{c}\text { No } \\
\text { opinion }\end{array}$ & Agree & $\begin{array}{c}\text { Agree } \\
\text { a lot }\end{array}$ \\
\hline & & $\square$ & & & \\
\hline & & $\square$ & & & $\square$ \\
\hline & & $\square$ & & & $\square$ \\
\hline & $\square$ & $\square$ & & $\square$ & $\square$ \\
\hline \multirow{5}{*}{$\begin{array}{l}\text { 5. It's no fun at all } \\
6 . \text { It gives me energy } \\
\text { 7. It makes me sad } \\
\text { 8. It's very pleasant }\end{array}$} & $\begin{array}{c}\text { Disagree } \\
\text { a lot }\end{array}$ & Disagree & $\begin{array}{c}\text { No } \\
\text { opinion }\end{array}$ & Agree & $\begin{array}{c}\text { Agree } \\
\text { a lot }\end{array}$ \\
\hline & & $\square$ & $\square$ & & $\square$ \\
\hline & 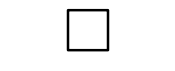 & $\square$ & & $\square$ & $\square$ \\
\hline & 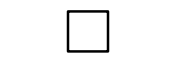 & $\square$ & & $\square$ & $\square$ \\
\hline & $\square$ & $\square$ & $\square$ & $\square$ & $\square$ \\
\hline \multirow{5}{*}{$\begin{array}{l}\text { 9. My body feels good } \\
\text { 10. I get something out of it } \\
\text { 11. It's very exciting } \\
\text { 12. It frustrates me }\end{array}$} & $\begin{array}{c}\text { Disagree } \\
\text { a lot }\end{array}$ & Disagree & $\begin{array}{c}\text { No } \\
\text { opinion }\end{array}$ & Agree & $\begin{array}{c}\text { Agree } \\
\text { a lot }\end{array}$ \\
\hline & & $\square$ & & & $\square$ \\
\hline & $\square$ & $\square$ & & $\square$ & $\square$ \\
\hline & $\square$ & $\square$ & & & $\square$ \\
\hline & $\square$ & $\square$ & & $\square$ & $\square$ \\
\hline \multirow{5}{*}{$\begin{array}{l}\text { 13. It's not at all interesting } \\
\text { 14. It gives me a strong } \\
\text { feeling of success } \\
\text { 15. It feels good } \\
\text { 16. I feel as though I would } \\
\text { rather be doing } \\
\text { something else }\end{array}$} & $\begin{array}{c}\text { Disagree } \\
\text { a lot }\end{array}$ & Disagree & $\begin{array}{c}\text { No } \\
\text { opinion }\end{array}$ & Agree & $\begin{array}{c}\text { Agree } \\
\text { a lot }\end{array}$ \\
\hline & $\square$ & $\square$ & $\square$ & $\square$ & $\square$ \\
\hline & $\square$ & $\square$ & & $\square$ & $\square$ \\
\hline & $\square$ & \begin{tabular}{|l|}
$\square$ \\
\end{tabular} & $\square$ & $\square$ & $\square$ \\
\hline & $\square$ & $\square$ & $\square$ & $\square$ & $\square$ \\
\hline
\end{tabular}




\section{Appendix I}

\section{Parental Influence Scale}

\section{Instructions:}

1. Circle the number that best describes how much you "strongly disagree", "disagree", "agree", or "strongly agree" with each statement.

2. Please choose only one answer.

3. Remember there are no right or wrong answers, simply choose the one that is best for you.

\begin{tabular}{|c|c|c|c|}
\hline Strongly & Disagree & Agree & Strongly \\
\hline Disagree & & & Agree \\
\hline 1 & 2 & 3 & 4 \\
\hline 1 & 2 & 3 & 4 \\
\hline 1 & 2 & 3 & 4 \\
\hline 1 & 2 & 3 & 4 \\
\hline 1 & 2 & 3 & 4 \\
\hline 1 & 2 & 3 & 4 \\
\hline 1 & 2 & 3 & 4 \\
\hline 1 & 2 & 3 & 4 \\
\hline 1 & 2 & 3 & 4 \\
\hline 1 & 2 & 3 & 4 \\
\hline
\end{tabular}




\section{Strongly Disagree Agree Strongly}

Disagree $\quad$ Agree

11. My parents like to walk for exercise.

1

2

3

4

12. My parents want me to play outside.

1

2

3

4

13. My parents do not like to do much physical

2

3

4 activity.

14. My parents tell me not to watch too much TV.

15. My parents usually walk or bike a lot.

1

2

3

4

16. My parents remind me to do some physical

2

3

4 activity.

17. My parents would rather walk to the store if 1

2

3

4 possible.

18. My parents encourage me to try hard at 1

2

3 games and sports. 


\section{Appendix J}

\section{Environment Scale}

1. At home, there are enough supplies and pieces of sports equipment (like balls, bicycles, skates) to use for physical activity.

$\square$ Disagree a lot $\quad \square$ Disagree $\quad \square$ No opinion $\quad \square$ Agree $\quad \square$ Agree a lot

2. There are playgrounds, parks, or gyms close to my home or that I can get easily.
Disagree a lot
Disagree
$\square$ No opinion
$\square$ Agree
Agree a lot

3. It is safe to walk or jog alone in my neighborhood during the day.
Disagree a lot
Disagree
$\square$ No opinion
$\square$ Agree
Agree a lot

4. It is difficult to walk or jog in my neighborhood because of things like traffic, no sidewalks, dogs, or gangs.

$\square$ Disagree a lot $\quad \square$ Disagree $\quad \square$ No opinion $\quad \square$ Agree $\quad \square$ Agree a lot

5. At school, there are playgrounds, gym spaces, and enough supplies (like balls, hula hoops) to use for physical activity.
$\square$ Disagree a lot
Disagree
$\square$ No opinion
Agree
Agree a lot 


\section{Appendix K}

Hierarchical Regression Analysis Evaluating Predictors of Physical activity $(N=133)$

\section{Self-reported Physical Activity Levels}

\begin{tabular}{|c|c|c|c|c|c|c|c|}
\hline Variable & Adjusted $R^{2}$ & $\Delta R^{2}$ & $F(d f)$ & $p$-value & $\beta$ & $t$-statistic & $p$-value \\
\hline Model 1 & .12 & .22 & $F(11,90)=2.28$ & .016 & & & \\
\hline Age & & & & & -.10 & -1.04 & .300 \\
\hline Sex & & & & & -.02 & -.24 & .814 \\
\hline Ethnicity & & & & & -.08 & -.71 & .480 \\
\hline Race & & & & & -.18 & -1.75 & .083 \\
\hline Marital status & & & & & .08 & .71 & .480 \\
\hline Family income & & & & & -.17 & -1.34 & .182 \\
\hline Number of children & & & & & .18 & 1.79 & .077 \\
\hline Father employment status & & & & & -.13 & -1.01 & .314 \\
\hline Mother employment status & & & & & -.05 & -.47 & .637 \\
\hline Mother education level & & & & & .35 & 3.00 & .004 \\
\hline Father education level & & & & & -.14 & -1.20 & .234 \\
\hline Model 2 & .35 & .23 & $F(5,85)=4.34$ & $<.0001$ & & & \\
\hline Age & & & & & -.08 & -.97 & .337 \\
\hline Sex & & & & & -.04 & -.43 & .669 \\
\hline Ethnicity & & & & & -.01 & -.10 & .920 \\
\hline Race & & & & & -.10 & -1.06 & .292 \\
\hline Marital status & & & & & .01 & .08 & .940 \\
\hline Family income & & & & & -.146 & -1.31 & .193 \\
\hline Number of children & & & & & .15 & 1.79 & .076 \\
\hline Father employment status & & & & & -.06 & -.51 & .614 \\
\hline
\end{tabular}




\begin{tabular}{|c|c|c|c|}
\hline Mother employment status & .01 & .09 & .931 \\
\hline Mother education level & .23 & 2.21 & .030 \\
\hline Father education level & -.15 & -1.42 & .159 \\
\hline Physical activity enjoyment & .25 & 2.46 & .016 \\
\hline Physical activity self-efficacy & .18 & 1.77 & .080 \\
\hline Parental support & .15 & 1.26 & .212 \\
\hline Parental role modeling & -.05 & -.49 & .624 \\
\hline Perceived environment & .13 & 1.16 & .250 \\
\hline
\end{tabular}

\section{Pedometer Steps}

\begin{tabular}{|c|c|c|c|c|c|c|c|}
\hline Variable & $\begin{array}{c}\text { Adjusted } \\
R^{2}\end{array}$ & $\Delta R^{2}$ & $F(d f)$ & $p$-value & $\beta$ & $t$-statistic & $p$-value \\
\hline Model 1 & .08 & .15 & $F(8,95)=2.12$ & .042 & & & \\
\hline Sex & & & & & -.26 & -2.62 & .010 \\
\hline Ethnicity & & & & & -.17 & -1.55 & .124 \\
\hline Marital status & & & & & -.03 & -.27 & .787 \\
\hline Family income & & & & & .08 & .68 & .496 \\
\hline Number of children & & & & & .18 & 1.77 & .079 \\
\hline Father employment status & & & & & -.04 & -.31 & .759 \\
\hline Mother employment status & & & & & -.24 & -2.15 & .034 \\
\hline Mother education level & & & & & .17 & 1.68 & .096 \\
\hline Model 2 & .10 & .07 & $F(13,90)=1.92$ & .038 & & & \\
\hline Sex & & & & & -.29 & -2.92 & .004 \\
\hline Ethnicity & & & & & -.15 & -1.31 & .193 \\
\hline Marital status & & & & & -.10 & -.79 & .432 \\
\hline Family income & & & & & .07 & .55 & .585 \\
\hline Number of children & & & & & .19 & 1.95 & .054 \\
\hline Father employment status & & & & & -.06 & -.52 & .603 \\
\hline
\end{tabular}




\begin{tabular}{lccc}
\hline Mother employment status & -.18 & -1.55 & .124 \\
Mother education level & .20 & 1.87 & .064 \\
Physical activity enjoyment & -.25 & -2.10 & .039 \\
Physical activity self-efficacy & .14 & 1.19 & .239 \\
Parental support & .05 & .36 & .718 \\
Parental role modeling & .15 & 1.18 & .239 \\
Perceived environment & .03 & .24 & .813 \\
\hline
\end{tabular}




\section{CURRICULUM VITAE}

Jiying Ling, PhD, MS, RN

210 Bontressa Drive Apt. \#1 Louisville Kentucky 40206

Phone: (502) 852-6946 (Office) Email: lingjiying@gmail.com

\section{EDUCATION}

Ph.D., Nursing

December 2013

University of Louisville

Research Area: Childhood Obesity Intervention (Healthy Behavior Promotion)

Dissertation: Psychosocial and environmental determinants of physical activity in

elementary school children: Implications for interventions to reduce childhood obesity

GPA: $3.97 / 4.0$

M.S., Biostatistics

May 2013

University of Louisville

Thesis: Preliminary assessment of a school-based healthy lifestyle program among rural children

GPA: $4.0 / 4.0$

M.S., Nursing (not completed)

September 2008-June 2009

Shandong University, China

Concentration: Critical Care Nursing

Credits Earned: 25.5

GPA: $89.7 / 100$

B.S., Nursing

July 2008

Shandong University, China

GPA: $85.1 / 100$

\section{APPOINTMENT}

Graduate Research Assistant

2009-Present

School of Nursing, University of Louisville

Graduate Teaching Assistant (Community Nursing Health) 2013 Fall

School of Nursing, University of Louisville

Statistics Tutor

2012 Fall

School of Nursing, University of Louisville 
School of Nursing, Shandong University, China

\section{GRANTS}

\section{Principal Investigator}

Psychosocial and environmental determinants of physical activity in elementary 2013 school children: Implications for interventions to reduce childhood obesity.

\section{Jiying Ling (PI)}

Funding: Commission on Diversity and Racial Equality Grant (\$1,000), Sigma Theta

Tau International-Iota Zeta Chapter Research \& Scholarship Award (\$500), Community

Engagement Grant (\$400), and Graduate Student Council Research Fund (\$300)

Exploring the needs and attitudes about pre-hospital care among college students. Jiying Ling (PI)

Funding: Shandong University Innovative Research Grant, \$200

\section{$\underline{\text { Research Assistant }}$}

Role Summary: Literature review, proposal preparation, IRB submission, data collection, data entry/analysis and output interpretation, poster/PowerPoint creation, and manuscript writing

Project BALANCE: Beneficial Activity Levels and Nutritional

2010-Present

Choices Everyday. Dr. Kristi King (PI)

Funding: Carol M. White Physical Education Program Grant (CFDA\# 84.215F), $\$ 1,400,000$

Fit into College II. Dr. Kristi King (PI)

$2011-2013$

Funding: Delphi Center awarded i2a SUN grant, University of Louisville, \$1,800

Evaluation of difference in select variable in rural/urban sample $2010-2013$ related to physical activity and low income women. Dr. Barbara Speck (PI)

Funding: Intramural Research Incentive Grant, University of Louisville, \$2,454

A feasibility study: Investigating a new theory of successful aging. Dr. Valeria McCarthy (PI)

Funding: Intramural Research Incentive Grant, University of Louisville, \$4,556 
Intervention research to assist families to adjust to caring for

$2010-2013$

persons with Dementia. Dr. Karen Robinson (PI)

Funding: Multiple funding resources, \$159,348

Older mothers and adult daughters: High blood pressure

2009-2011

self-management behaviors. Dr. Celeste Shawler (PI)

Funding: NIH/NINR Grant (1K01NR010239-01A1), \$261,905

\section{JOURNAL PEER REVIEWER}

Journal of Nursing Measurement

2013-Present

Nursing: Research and Reviews

2012-Present

Open Access Medical Statistics

2012-Present

\section{PUBLICATIONS}

Ling, J., King, K., Speck, B., Kim, S., \& Wu, D. (in press). Preliminary assessment of a school-based healthy lifestyle intervention among rural children. Journal of School Health.

Ling, J., Hines-Martin, V., \& Ji, H. (in press). Childhood development cross culturally: Implications for designing childhood obesity interventions and providing culturally competent care. Journal of Qilu Nursing (China).

Ling, J., Anderson, L. M., \& Ji, H. (in press). Self-management training for Chinese children with metabolic syndrome: Effectiveness and implications for school health. School Psychology International.

Ji, H., Ling, J., \& McCarthy, V. (in press). Successful aging in the United States and China: A theoretical basis to guide nursing research, practice, and policy. Journal of Transcultural Nursing.

Ridner, L., Ostapchuk, M., Cloud, R., Myers, J., Jorayeva, A., \& Ling, J. (in press). Using motivational interviewing for smoking cessation in primary care. Southern Medical Journal.

Ji, H., \& Ling, J. (2013). A concept analysis of successful aging: Perspectives for nursing. Journal of Nursing (China), 20(10A), 1-6.

McCarthy, V. L., Ling, J., \& Carini, R. M. (2013). The role of self-transcendence: A missing variable in the pursuit of successful aging? Research in Gerontological Nursing, 6(3), 178-186. doi: 10.3928/19404921-20130508-01 
King, K., Ling, J., Ridner, L., Jacks, D., Newton, K., \& Topp, R. (2013). Fit into College II: Physical activity and nutrition behavior effectiveness and programming recommendations. Recreational Sports Journal, 37(1), 29-41.

Ling, J., King, K., Speck, B., Wu, D., \& Kim, S. (2013). Preliminary assessment of a school-based healthy lifestyle program among rural children: Project BALANCE. Annals of Behavioral Medicine, 45(Abstract Supplement), s28.

Wang, X., \& Ling, J. (2012). Multiple valued logic approach for matching patient records in multiple databases. Journal of Biomedical Informatics, 45, 224-230. doi:10.1016/j.jbi.2011.10.009

Ling, J., Liu, J., Liu, C., Zou, F., \& Wang, K. (2009). Knowledge and attitudes about pre-hospital care among college students. Chinese Nursing Research, 24(2), 79-81.

\section{PRESENTATIONS}

\section{$\underline{\text { National }}$}

Ling, J., King, K., Speck, B., Wu, D., \& Kim, S. Preliminary assessment of a schoolbased healthy lifestyle program among rural children: Project BALANCE (Oral Presentation as a Top MHBC-related Student Research). The $34^{\text {th }}$ Annual Meeting \& Scientific Sessions of the Society of Behavioral Medicine, San Francisco, CA. March 20-23, 2013.

Ling, J., Jacks, D. E., King, K., Ridner, S. L., Conte, K., \& Topp, R. Effects of a 10week fitness program on fitness and perceptions of nutrition and physical activity among overweight and normal weight college students. NINR's $25^{\text {th }}$ Anniversary Concluding Scientific Symposium, Washington, DC. October 13, 2011.

\section{$\underline{\text { Regional }}$}

Speck, J. B., \& Ling, J. Barriers to regular physical activity in rural and urban lowincome women (Poster Discussion Presentation). Midwest Nursing Research Society 37th Annual Conference, Chicago, IL. March 7-10, 2013.

Ling, J., King, K., Speck, B., Wu, D., \& Kim, S. Preliminary assessment of a schoolbased healthy lifestyle program among rural children (Top Student Poster Discussion Presentation). Southern Nursing Research Society $27^{\text {th }}$ Annual Conference, Little Rock, Arkansas. February 27- March 2, 2013.

Jackson, B. M., Lehna, C., Alaradi, M., \& Ling, J. Differences in children's home safety practices between families living in rural and urban areas. Southern Nursing Research Society 27th Annual Conference, Little Rock, Arkansas. February 27March 2, 2013. 
Ling, J., \& Speck, J. B. Perceived barriers to physical activity in rural and urban lowincome women. Midwest Nursing Research Society $36^{\text {th }}$ Annual Conference, Dearborn, Michigan. April 12-15, 2012.

Ling, J., Alaradi, M., Jackson, B., \& Lehna, C. What baccalaureate registered nursing students found out from a home safety assignment. Southern Nursing Research Society $26^{\text {th }}$ Annual Conference, New Orleans, Louisiana. February 22-25, 2012.

Speck, J. B., \& Ling, J. Descriptive study of participants attending a health fair in a low income urban neighborhood. Midwest Nursing Research Society $35^{\text {th }}$ Annual Conference, Columbus, Ohio. March 24-27, 2011.

Ling, J., \& Shawler, C. Body mass index is associated with health related quality of life among older mother-adult daughter dyad. Southern Nursing Research Society $25^{\text {th }}$ Annual Conference, Jacksonville, Florida. February 16-19, 2011.

\section{$\underline{\text { Local }}$}

Ling, J., Wen, F., \& Speck, J. Psychosocial determinants of physical activity in elementary school children: Private vs. Public. Research! Louisville, University of Louisville, Louisville, KY. September 24-27, 2013

Watts, S., Chlebowy, D., Kubiak, N., Batsha, C., \& Ling, J. The impact of depression, anxiety, and stress on diabetes markers and self-management behaviors in African American adults with type 2 diabetes. Summer Undergraduate Research-Summer Research Opportunity Program, University of Louisville, Louisville, KY. July 31, 2013.

Massouda J., \& Ling, J. Empowerment of charge nurses: A key factor for achieving nursing excellence and ANCC Magnet designation. University of Louisville Hospital Research Day, Louisville, KY. May 9, 2013.

Ling, J., \& Speck, B. Perceived barriers to physical activity in rural and urban lowincome women. Research! Louisville, University of Louisville, Louisville, KY. October 11, 2011.

Lehna, C., Jackson, B., Ling, J., \& Alaradi, M. What baccalaureate registered nursing students found out from a home safety assignment. Research! Louisville, University of Louisville, Louisville, KY. October 11, 2011.

Ling, J., Jacks, D., King, K., Ridner, S. L., Conte, K., \& Topp, R. Effects of a 10-week program on fitness and perceptions of nutrition and physical activity among overweight and normal weight college students. Community Engaged Research: Community Diabetes Prevention Program in Kentuckiana, University of Louisville, Louisville, KY. April 14, 2011. 
Ling, J., \& Shawler, C. Body mass index is associated with health related quality of life among older mother-adult daughter dyad. Community Engaged Research: Community Diabetes Prevention Program in Kentuckiana, University of Louisville, Louisville, KY. April 14, 2011.

Ling, J., Jacks, D., King, K., Ridner, S. L., Conte, K., \& Topp, R. Effects of a 10-week program on fitness and perceptions of nutrition and physical activity among overweight and normal weight college students. Graduate Student Council, University of Louisville, Louisville, KY. April 1, 2011.

Ling, J., \& Shawler, C. Body mass index is associated with health related quality of life among older mother-adult daughter dyad. Graduate Student Council, University of Louisville, Louisville, KY. April 1, 2011.

Ling, J., \& Shawler, C. Body mass index is a risk factor for health related quality of life among older mother-adult daughter dyads. Research! Louisville, University of Louisville, Louisville, KY. October 11-15, 2010.

\section{TEACHING}

NURS 361: Community Health Nursing 2013 Fall Guest lectures on cultural diversity, health promotion and epidemiology (80 students) Clinical instructor for community practice (10 students) Attended a two-day Blackboard Training in June 2013

HSS 604: Research Methods 2013 Spring Guest Lecturer, 22 graduate students from sports administration, exercise physiology and community health Two 3-hour lectures related to data analysis, Thesis Chapter 4 (Results) and Chapter 5 (Discussion) writing

PHST 600: Introduction to Biostatistics for Public Health 2012 Fall Tutor, 12 graduate nursing students 3-hour lecture per week plus two 5-hour mid-term and final exam review sessions

\section{HONORS AND AWARDS}

Research!Louisville Poster Presentation Winner Award 2013 University of Louisville

IdeaFestival Scholar

Louisville, Kentucky

Top Student Poster Presentation Award 2013 Southern Nursing Research Society 
PhD Program Travel Fund (\$300)

School of Nursing, University of Louisville

Graduate Student Council (GSC) Travel Fund (\$350)

2013

University of Louisville

Ruth Craddock Research Fund (\$500)

2012

School of Nursing, University of Louisville

Who's Who among Students in American Universities \& Colleges

2012

in recognition of outstanding merit and accomplishment as a student at University of

Louisville

Outstanding Adult Learner Award for Graduate Student

2012

Kentuckiana Metroversity, Kentucky

Ruth Craddock Research Fund (\$500)

2011

School of Nursing, University of Louisville

\section{PROFESSIONAL MEMBERSHIPS}

Society of Behavioral Medicine

2013-Present

Honor Society of Nursing Sigma Theta Tau International

2011-Present

Southern Nursing Research Society

2011-Present

Abstract Reviewer for SNRS 27 Annual Conference, 2013

Midwest Nursing Research Society

Abstract Reviewer for MNRS $38^{\text {th }}$ Annual Conference, 2014

2011-Present

NATIONAL BOARD CERTIFICATE AND STATE RN LICENSURE

California (RN), USA

August 2013-May 2015

License No: 850466

Shandong (RN), China

March 2010-March 2015

License No: 201037002002

Certificate of General Management Proficiency

July 2007-Present

China Employment Training Technical Instruction Center

Certificate No: 20071031501400093

First Aider Certificate

2005- Present

Red Cross Society of China

Certificate No: 20050096 


\section{CLINICAL PRACTICE}

First Affiliated Hospital of Dalian Medical University, China

$2007-2008$

\section{COMMITTEES AND SERVICES}

Sigma Theta Tau International - Iota Zeta Chapter, Biennial Conference Delegate, Electronic Delegate, and Research Committee

2013-Current

Wellington Elementary School, School Wellness Policy, Evaluator 2012

Indiana/Kentucky Tornado Relief, Volunteer 2012

University of Louisville, Department of Mathematics, Tutor 2011

Association of Graduate Nursing Students, Treasurer

2009-Current University of Louisville

China, Sichuan Earthquake Relief, Volunteer

May 2008 